\title{
Visible-light Induced Direct Synthesis of Polysubstituted furans from Cyclopropyl ketones
}

\author{
Liyan Feng, Hang Yan, Chao Yang, Dafa Chen and Wujiong Xia*
}

State Key Lab of Urban Water Resource and Environment, Shenzhen Graduate School, Harbin Institute of Technology, Harbin

150080

E-mail: xiawj@hit.edu.cn

Supporting information

Table of contents

1. Cyclic Voltammetry of $\mathbf{1 h}$

S 2

2. Optimization of conditions

S 2-4

3. The X-ray structure of $\mathbf{5 a}$

S 5-6

4. The ${ }^{1} \mathrm{H},{ }^{13} \mathrm{C}$ and ${ }^{19} \mathrm{~F}$ NMR spectra

S 7-90 


\section{Cyclic Voltammetry of (4-bromophenyl)-2-(4-methoxyphenyl)cyclopropylmethanone (1h)}

Cyclic Voltammetry was performed on CHI Instruments electrochemical workstation model: CHI 660D.

$1 \mathbf{h}(33 \mathrm{mg}, 0.1 \mathrm{mmol})$ was dissolved in the anhydrous acetonitrile solution of $\mathrm{NBu}_{4} \mathrm{PF}_{6}(1 \mathrm{mmol} / \mathrm{ml}, 20 \mathrm{ml})$. The solution was degassed by bubbling with $\mathrm{N}_{2}$ for ten minutes. An irreversible oxidation peak was observed at $+0.75 \mathrm{~V}$ vs. SCE.

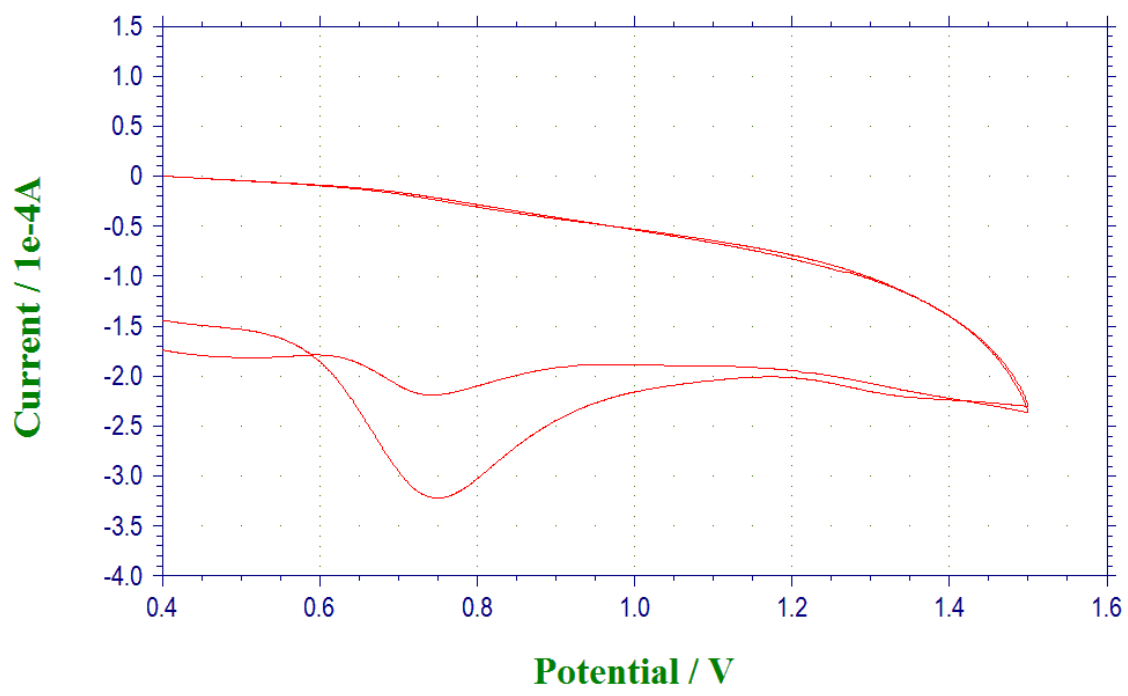

Sept. 17, 2015 15:48:23 Tech: CV File: Smooth Unsaved

Init $E(V)=-2$

High $E(V)=1.5$

Low $E(V)=-2$

Scan Rate $(V / s)=0.05$

Segment $=4$

Smpl Interval $(V)=0.001$

Sensitivity (AV) $=0.001$

Segment 1:

Segment 2

Segment 4

Potential / V

\section{Optimization of conditions for synthesis of 2,5-diarylfurans by photocatalysis}

Table S1. Effect of oxidation system

\begin{tabular}{|c|c|c|c|c|}
\hline Entry & Oxidant & Addition & Time(h) & Yield(\%) \\
\hline 1 & $\mathrm{CBr}_{4}$ & $\mathrm{CF}_{3} \mathrm{SO}_{2} \mathrm{Na}$ & 24 & 10 \\
\hline 2 & $\mathrm{Na}_{2} \mathrm{~S}_{2} \mathrm{O}_{8}$ & $\mathrm{CF}_{3} \mathrm{SO}_{2} \mathrm{Na}$ & 48 & $<5$ \\
\hline 3 & $\mathrm{Na}_{2} \mathrm{~S}_{2} \mathrm{O}_{8}$ & $\mathrm{LiBr}$ & 24 & 30 \\
\hline 4 & $\mathrm{Na}_{2} \mathrm{~S}_{2} \mathrm{O}_{8}$ & $\mathrm{LiCl}$ & 48 & 0 \\
\hline 5 & $\mathrm{Na}_{2} \mathrm{~S}_{2} \mathrm{O}_{8}$ & $\mathrm{KI}$ & 48 & 0 \\
\hline 6 & $\mathrm{MV}\left(\mathrm{PF}_{6}\right)_{2}$ & none & 48 & 0 \\
\hline 7 & $\mathrm{CBr}_{4}$ & none & 24 & 15 \\
\hline 8 & $\mathrm{CBr}_{4}$ & LiBr & 24 & 60 \\
\hline 9 & $\mathrm{CBr}_{4}$ & $\mathrm{FeCl}_{3}$ & 24 & $<5$ \\
\hline 10 & $\mathrm{O}_{2}$ & $\mathrm{LiBr}$ & 48 & 0 \\
\hline 11 & $\mathrm{BrCH}(\mathrm{COOEt})_{2}$ & $\mathrm{LiBF}_{4}$ & 48 & 0 \\
\hline
\end{tabular}


Table S2. Effect of solvent system<smiles>COc1ccc(C2CC2C(=O)c2ccccc2)cc1</smiles><smiles>COc1ccc(-c2ccc(-c3ccccc3)o2)cc1</smiles>

\begin{tabular}{cccc}
\hline Entry & Solvent & Time $(\mathrm{h})$ & Yield(\%) \\
\hline $\mathbf{1}$ & $\mathbf{C H}_{3} \mathbf{C N}$ & $\mathbf{2 4}$ & $\mathbf{6 0 \%}$ \\
2 & $\mathrm{CH}_{3} \mathrm{NO}_{2}$ & 24 & $17 \%$ \\
3 & $\mathrm{DMF} / \mathrm{DMSO} / \mathrm{MeOH}$ & 48 & 0 \\
4 & $\mathrm{CH}_{2} \mathrm{Cl}_{2} /$ Acetone $/ \mathrm{CHCl}_{3}$ & 48 & 0 \\
\hline
\end{tabular}

Table S3. Effect of photocatalyst system<smiles>COc1ccc(C2CC2C(=O)c2ccccc2)cc1</smiles><smiles>COc1ccc(-c2ccc(-c3ccccc3)o2)cc1</smiles>

\begin{tabular}{ccccc}
\hline Entry & Photocatalyst & Dosage $(\%)$ & Time $(\mathrm{h})$ & Yield $(\%)$ \\
\hline 1 & $\mathrm{Ru}(\mathrm{bpy})_{3} \mathrm{Cl}_{2} \cdot 3 \mathrm{H}_{2} \mathrm{O}$ & 5 & 24 & 60 \\
2 & $\mathrm{Ir}(\mathrm{ppy})_{2}(\mathrm{dtbbpy})\left(\mathrm{PF}_{6}\right)$ & 5 & 24 & 32 \\
3 & $f a c-\mathrm{Ir}(\mathrm{ppy})_{3}$ & 5 & 24 & 20 \\
4 & Eosin $\mathrm{Y}$ & 5 & 50 & 23 \\
$\mathbf{5}$ & $\mathbf{R u}(\mathbf{d t b b p y})_{3}\left(\mathbf{P F}_{6}\right)_{2}$ & $\mathbf{5}$ & $\mathbf{1 2}$ & $\mathbf{7 8}$ \\
6 & $\mathrm{Ru}(\mathrm{dtbbpy})_{3}\left(\mathrm{PF}_{6}\right)_{2}$ & 1 & 20 & 74 \\
7 & None & 0 & 120 & $<10$ \\
\hline
\end{tabular}

Table S4. Effect of molarity of substrates<smiles>COc1ccc(C2CC2C(=O)c2ccccc2)cc1</smiles>

$1 \mathrm{a}$

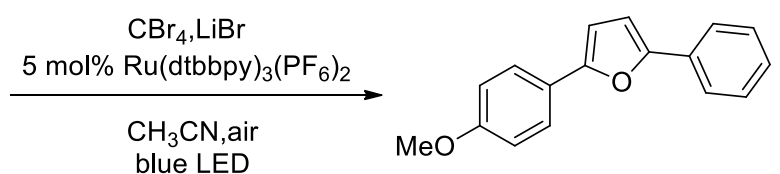

$4 a$

\begin{tabular}{cccccc}
\hline Entry & $\mathbf{1 a}(\mathrm{M})$ & $\mathrm{CBr}_{4}$ (equiv.) & $\operatorname{LiBr}($ equiv.) & Time(h) & Yield(\%) \\
\hline 1 & 0.1 & 1 & 0 & 10 & 10 \\
2 & 0.1 & 1 & 0.5 & 10 & 20 \\
3 & 0.05 & 1 & 0 & 10 & 33 \\
4 & 0.05 & 1 & 0.5 & 10 & 65 \\
$\mathbf{5}$ & $\mathbf{0 . 0 5}$ & $\mathbf{0 . 5}$ & $\mathbf{1}$ & $\mathbf{2 0}$ & $\mathbf{7 8}$ \\
\hline
\end{tabular}

Table S5. Screening of conditons for bromofuran sysnthesis.<smiles>COc1ccc(C2CC2C(=O)c2ccccc2)cc1</smiles>

$1 \mathrm{a}$<smiles>COc1ccc(-c2oc(-c3ccccc3)cc2Br)cc1</smiles>

$5 a$ 


\begin{tabular}{cccccc}
\hline Entry & $\mathbf{1 a}(\mathrm{M})$ & $\mathrm{CBr}_{4}$ (equiv.) & Conversion(\%) & Time(h) & Yield(\%) \\
\hline 1 & 0.05 & 0.5 & 99 & 24 & 0 \\
2 & 0.05 & 0.6 & 100 & 24 & $<5$ \\
3 & 0.05 & 1 & 100 & 30 & 30 \\
4 & 0.05 & 1.5 & 100 & 50 & 43 \\
$\mathbf{5}$ & $\mathbf{0 . 0 5}$ & $\mathbf{2 . 5}$ & $\mathbf{1 0 0}$ & $\mathbf{5 0}$ & $\mathbf{5 4}$ \\
\hline
\end{tabular}

Table S6. Screening of conditons for carbethoxy furan sysnthesis.<smiles>CCOC(=O)C1C(C(=O)c2ccccc2)C1c1ccc(OC)cc1</smiles>

$6 a$

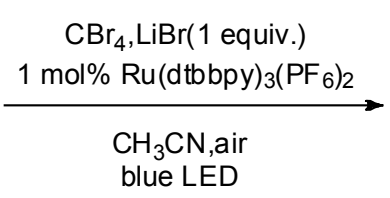
blue LED

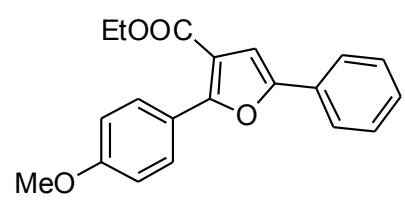

$7 a$

\begin{tabular}{cccccc}
\hline Entry & $\mathbf{1 a}(\mathrm{M})$ & $\mathrm{CBr}_{4}$ (equiv.) & Conversion(\%) & Time(h) & Yield(\%) \\
\hline 1 & 0.05 & 0.5 & 0 & 48 & 0. \\
2 & 0.05 & 1 & 0 & 48 & 0. \\
$\mathbf{3}$ & $\mathbf{0 . 0 5}$ & $\mathbf{2}$ & $\mathbf{1 0 0}$ & $\mathbf{3 4}$ & $\mathbf{9 6}$ \\
4 & 0.05 & 2.5 & 100 & 30 & 90 \\
\hline
\end{tabular}<smiles>CCOC(=O)C1C(C(=O)C(C)(C)C)C1c1ccc(OC)cc1</smiles>

6j

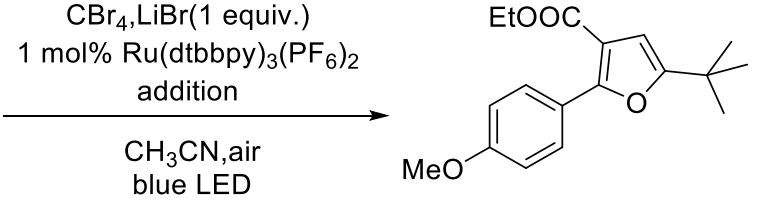

$7 \mathbf{j}$

\begin{tabular}{ccccc}
\hline Entry & $\mathrm{CBr}_{4}$ (equiv.) & Acid & Conversion $(\%)$ & Yield $(\%)$ \\
\hline 1 & 0.5 & None & 0 & 0 \\
2 & 1 & $\mathrm{CH}_{3} \mathrm{COOH}(20 \mathrm{~mol} \%)$ & 0 & 0 \\
3 & 1 & $\mathrm{HCl}(\mathrm{conc} .20 \mathrm{~mol} \%)$ & 100 & 20 \\
4 & 1 & $p$-TSA $\cdot \mathrm{H}_{2} \mathrm{O}(10 \mathrm{~mol} \%)$ & 0 & 0 \\
$\mathbf{5}$ & $\mathbf{1}$ & $\boldsymbol{p}$-TSA $\cdot \mathrm{H}_{\mathbf{2}} \mathbf{O}(\mathbf{2 0} \mathbf{m o l} \%)$ & $\mathbf{1 0 0}$ & $\mathbf{9 5}$ \\
6 & 0.5 & $p$-TSA $\cdot \mathrm{H}_{2} \mathrm{O}(20 \mathrm{~mol} \%)$ & 50 & 35 \\
\hline
\end{tabular}




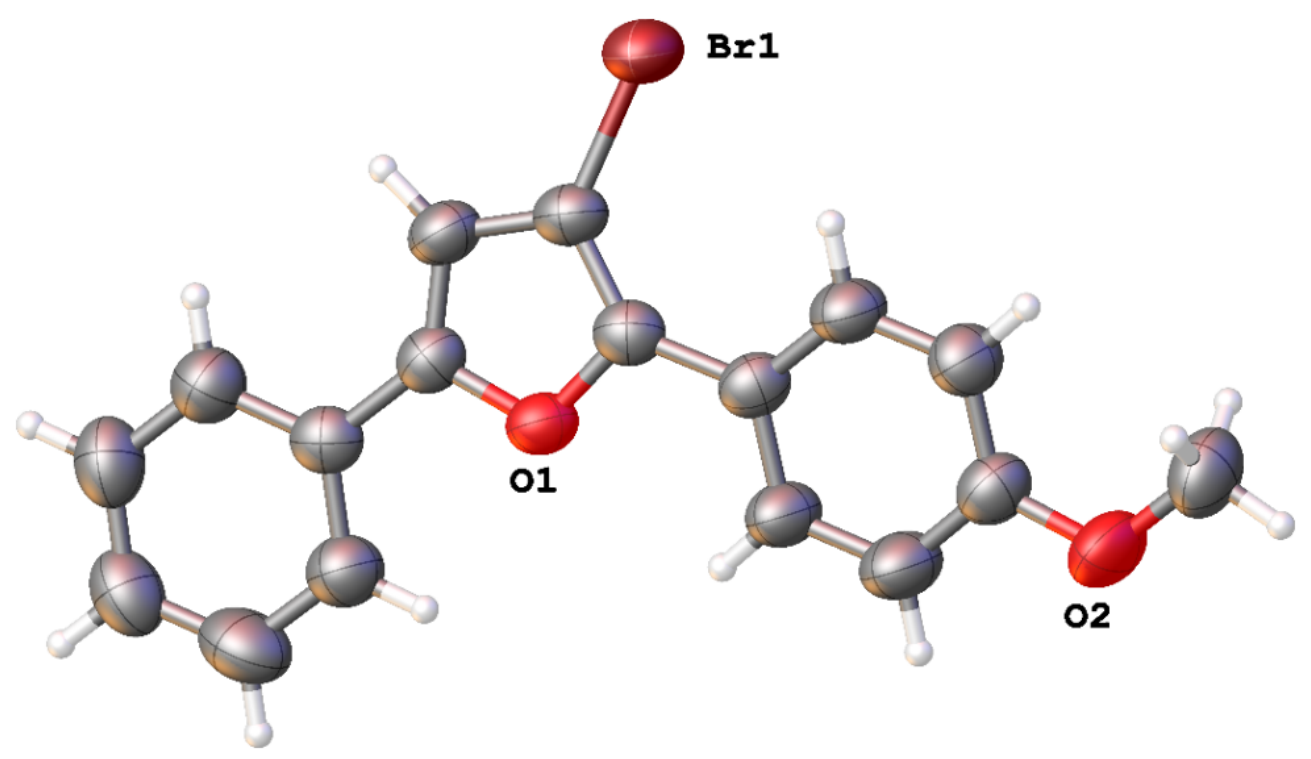

The X-ray structure of 5a. The ellipsoid contour percent probability level is $50 \%$.

\section{Experimental}

Single crystals of $\mathbf{5 a}$ were recrystallized in $\mathrm{MTBE} / \mathrm{MeOH}=5: 1$. A suitable crystal was selected and operated on a SuperNova, Dual, Cu at zero, Eos diffractometer. The crystal was kept at 293(2) K during data collection. Using Olex $2^{1}$, the structure was solved with the SIR2004 structure solution program using Direct Methods and refined with the $\mathrm{XH}^{2}$ refinement package using CGLS minimisation. 
Table S7. Crystal data and structure refinement for $\mathbf{5 a}$

\begin{tabular}{|c|c|}
\hline Formula & $\mathrm{C}_{17} \mathrm{H}_{13} \mathrm{BrO}_{2}$ \\
\hline Formula weight & 329.18 \\
\hline $\mathrm{T}(\mathrm{K})$ & $293(2)$ \\
\hline Wavelength & $0.71073 \AA$ \\
\hline Crystal system & triclinic \\
\hline Space group & $\mathrm{P} \overline{1}$ \\
\hline$a(\AA)$ & $5.5202(2)$ \\
\hline$b(\AA)$ & $13.6425(5)$ \\
\hline$c(\AA)$ & $20.1076(9)$ \\
\hline$\alpha\left({ }^{\circ}\right)$ & $107.956(4)$ \\
\hline$\beta\left({ }^{\circ}\right)$ & $91.404(4)$ \\
\hline$\gamma\left({ }^{\circ}\right)$ & $90.120(3)$ \\
\hline Volume & $1440.03(10)$ \\
\hline $\mathrm{Z}$ & 4 \\
\hline $\mathrm{D}\left(\mathrm{g} / \mathrm{cm}^{3}\right)$ & 1.518 \\
\hline Crystal size $/ \mathrm{mm}^{3}$ & $0.2 \times 0.136 \times 0.055$ \\
\hline $\mathrm{F}(000)$ & 664.0 \\
\hline $2^{\Theta}$ range for data collection $\left({ }^{\circ}\right)$ & 5.976 to 50.698 \\
\hline Index ranges & $-6 \leq \mathrm{h} \leq 6,-16 \leq \mathrm{k} \leq 16,-24 \leq 1 \leq 24$ \\
\hline$\mu\left(\mathrm{mm}^{-1}\right)$ & 2.852 \\
\hline Reflections collected & 9568 \\
\hline Independent reflections & $5267\left[\mathrm{R}_{\mathrm{int}}=0.0268, \mathrm{R}_{\text {sigma }}=0.0421\right]$ \\
\hline Data/restraints/parameters & $5267 / 0 / 363$ \\
\hline Goodness-of-fit on $F^{2}$ & 1.078 \\
\hline Final R indexes $[\mathrm{I}>=2 \sigma(\mathrm{I})]$ & $\mathrm{R}_{1}=0.0669, \mathrm{wR}_{2}=0.1680$ \\
\hline Final $\mathrm{R}$ indexes [all data] & $\mathrm{R}_{1}=0.0874, \mathrm{wR}_{2}=0.1843$ \\
\hline Largest diff. peak/hole / e $\AA^{-3}$ & $1.32 /-0.36$ \\
\hline
\end{tabular}

\section{References:}

1. Dolomanov, O.V., Bourhis, L.J., Gildea, R.J, Howard, J.A.K. \& Puschmann, H. (2009), J. Appl. Cryst. 42, 339-341.

2. Sheldrick, G.M. (2015). Acta Cryst. C71, 3-8. 
3. The ${ }^{1} \mathrm{H}$ and ${ }^{13} \mathrm{C}$ NMR spectra of compounds

$\sqrt{\sqrt{10}}$

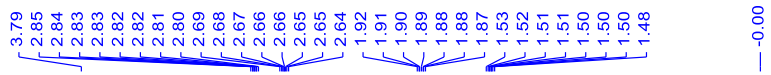<smiles>COc1ccc(C2CC2C(=O)c2ccccc2)cc1</smiles>

$1 a$

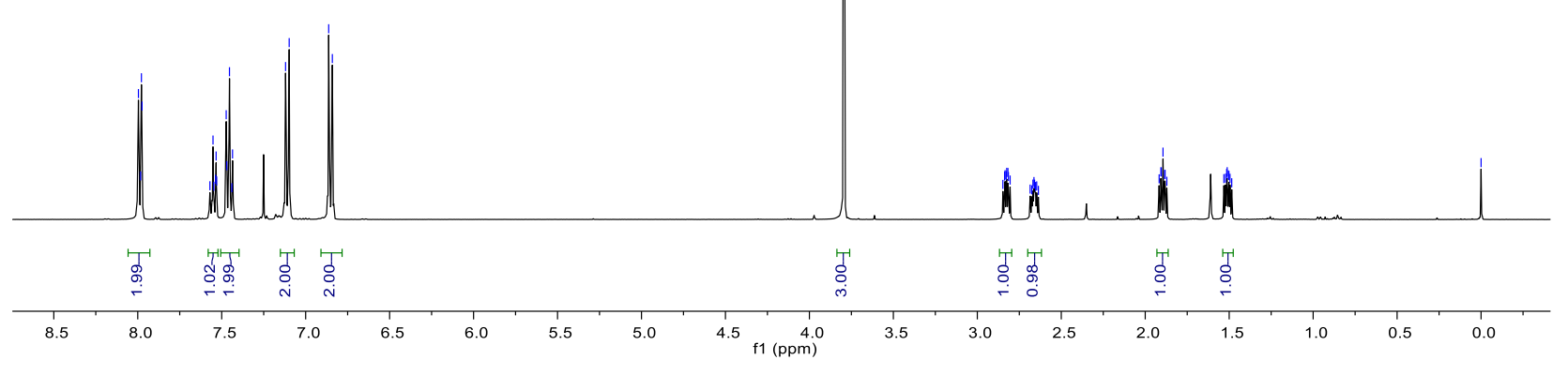

i

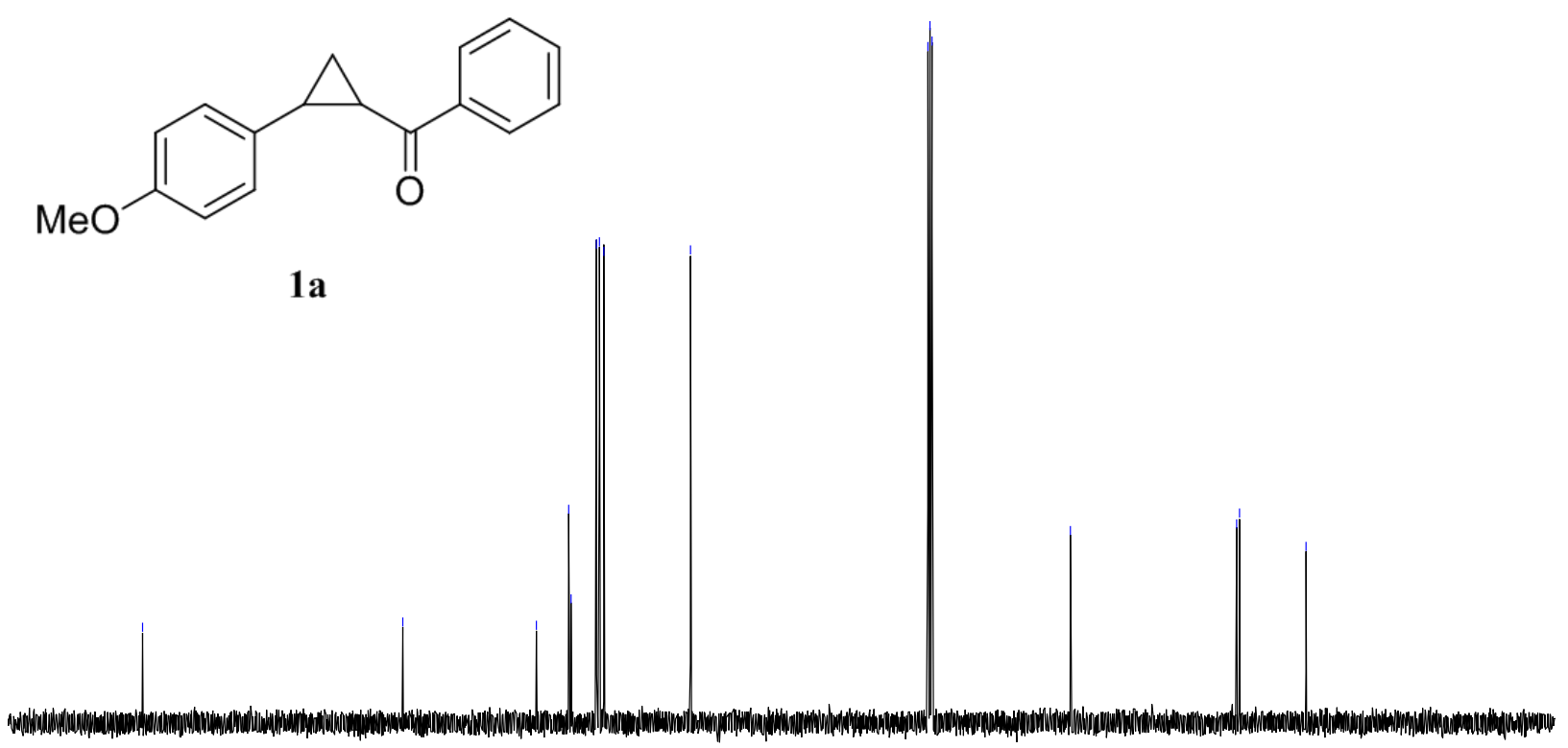

\begin{tabular}{lllllllllllllllllllllll}
\hline & 1 \\
210 & 200 & 190 & 180 & 170 & 160 & 150 & 140 & 130 & 120 & 110 & 100 & 90 & 80 & 70 & 60 & 50 & 40 & 30 & 20 & 10 & 0 & -10
\end{tabular} 

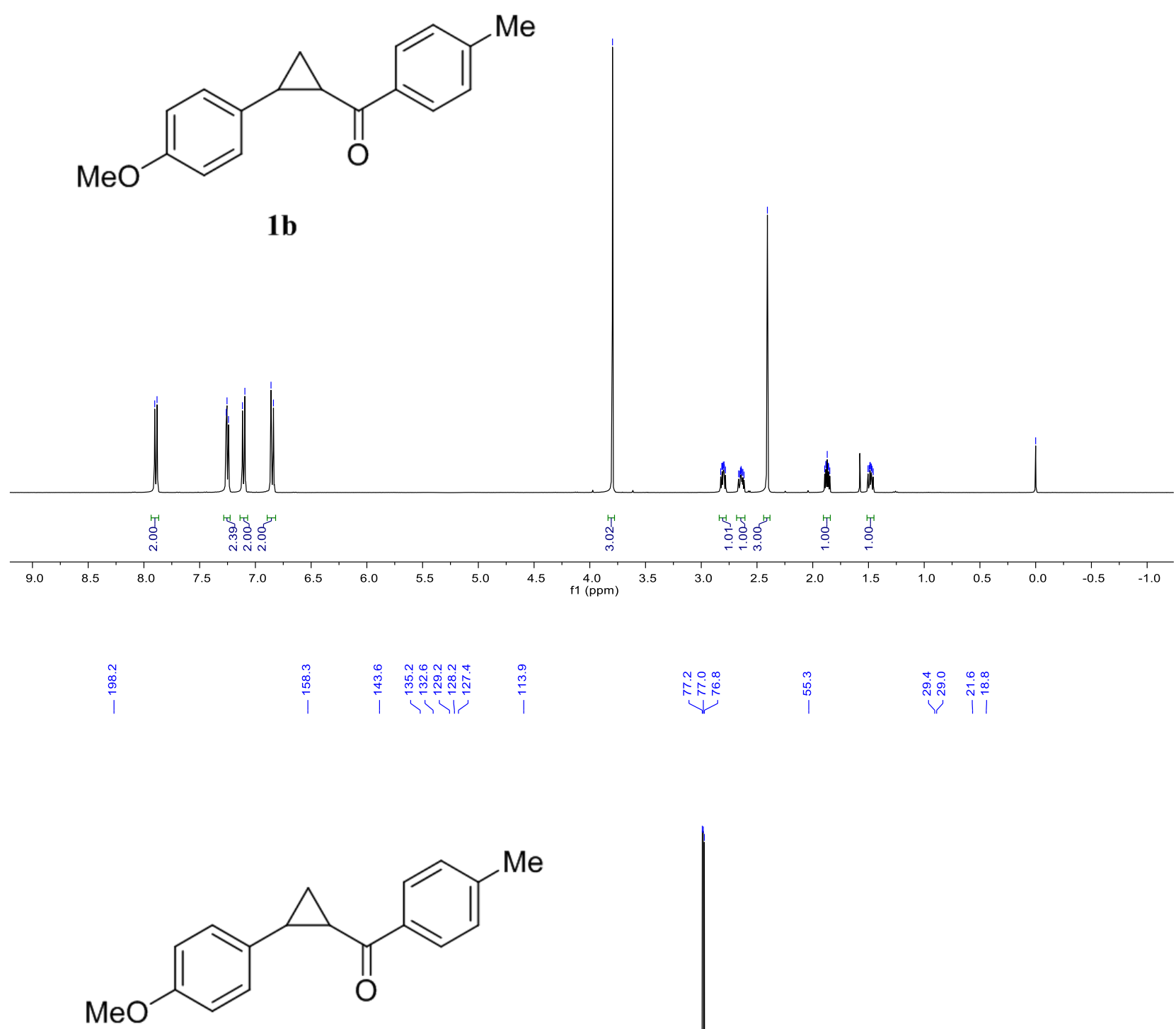

1b

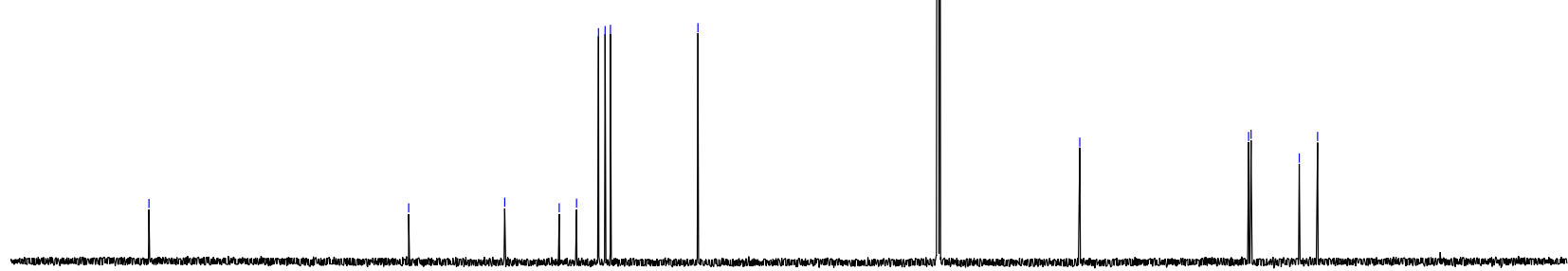

$\begin{array}{rlllllllllllllllllllllllll}\mid & 1 \\ 210 & 200 & 190 & 180 & 170 & 160 & 150 & 140 & 130 & 120 & 110 & 100 & 90 & 80 & 70 & 60 & 50 & 40 & 30 & 20 & 10 & 0 & -10\end{array}$ 

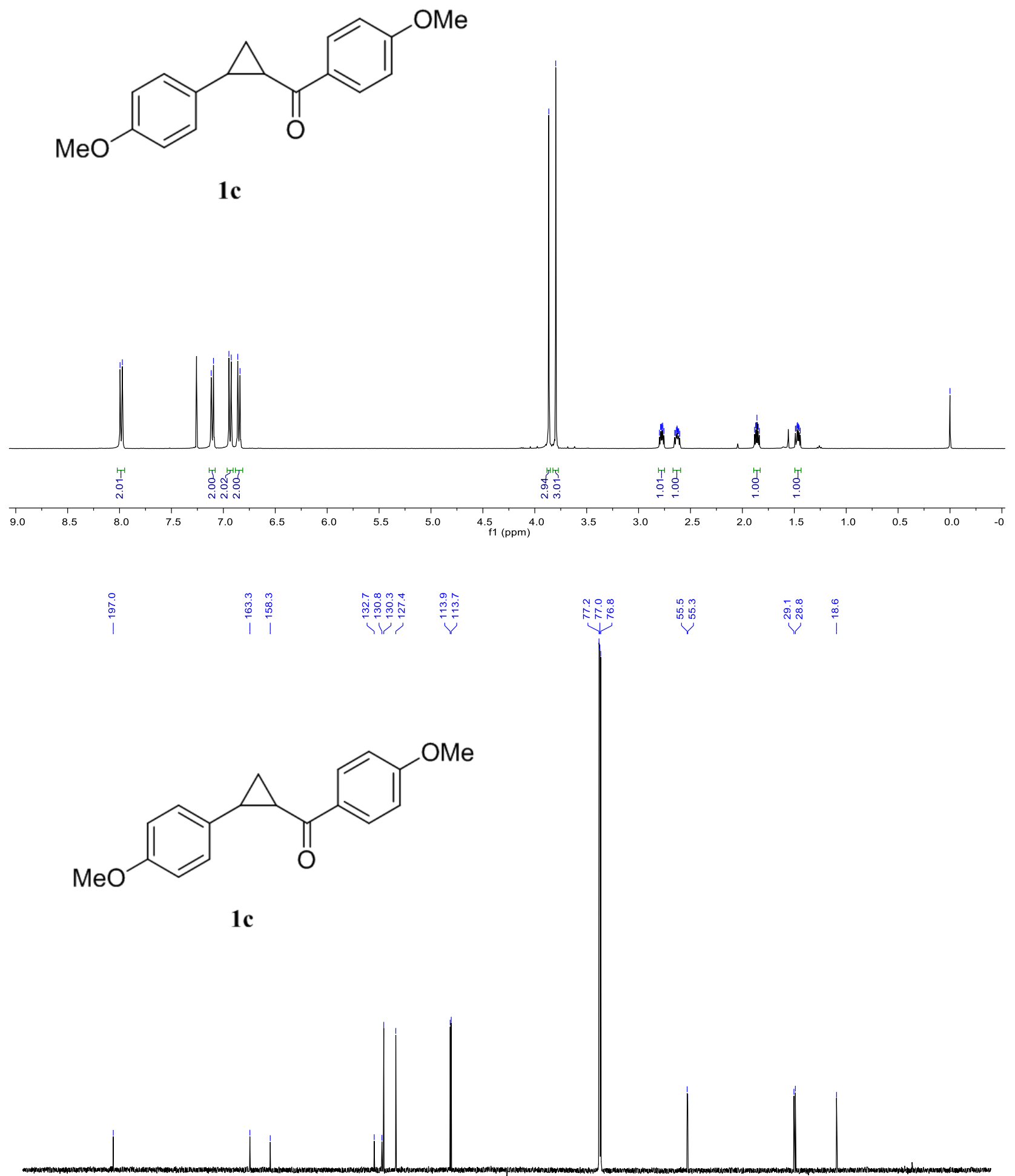

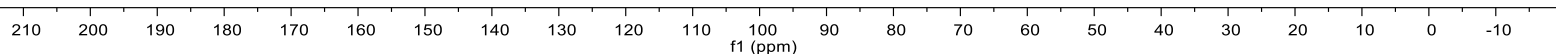


<smiles>COc1ccc(C2CC2C(=O)c2ccc(C(C)(C)C)cc2)cc1</smiles>

1d

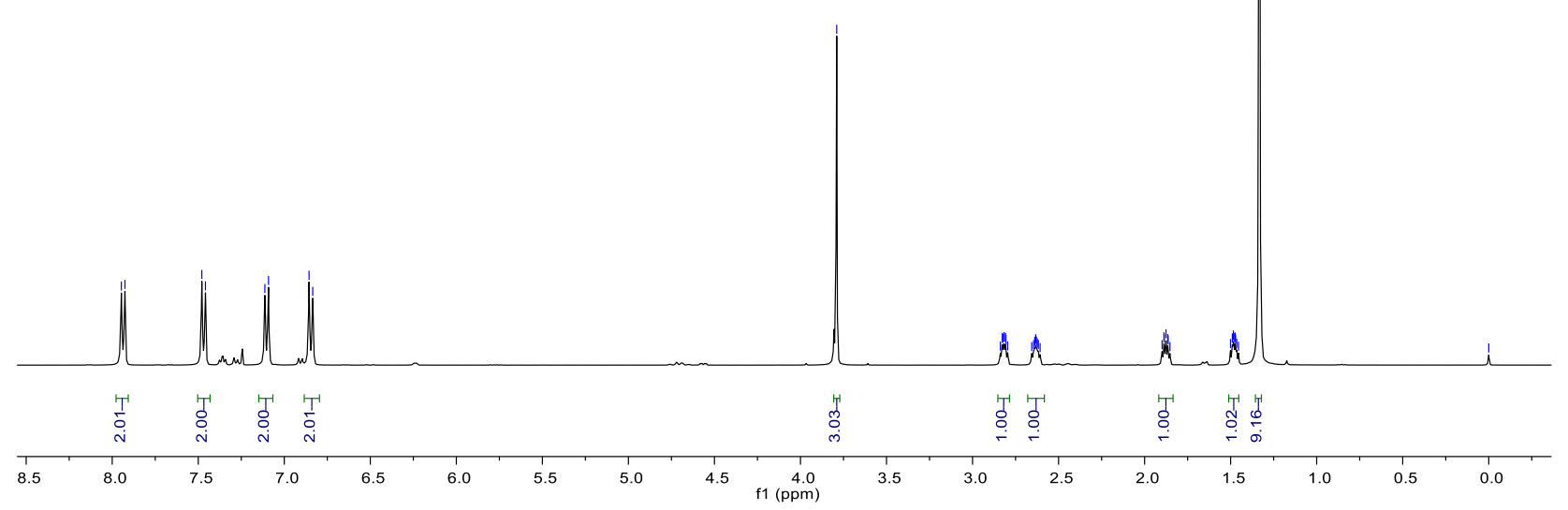

I<smiles>COc1ccc(C2CC2C(=O)c2ccc(C(C)(C)C)cc2)cc1</smiles>

1d

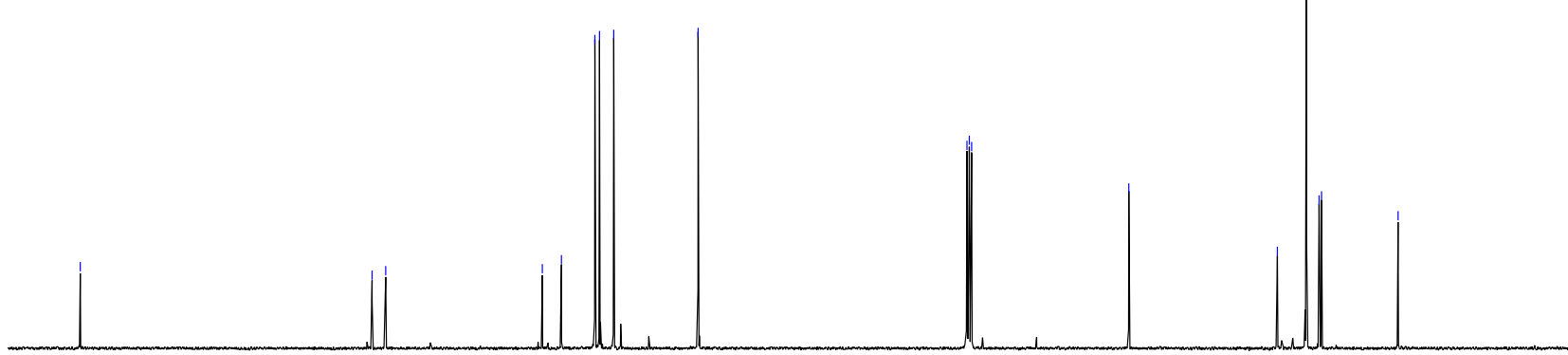

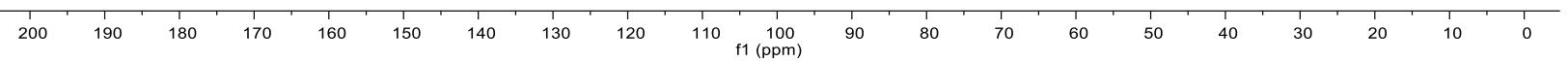




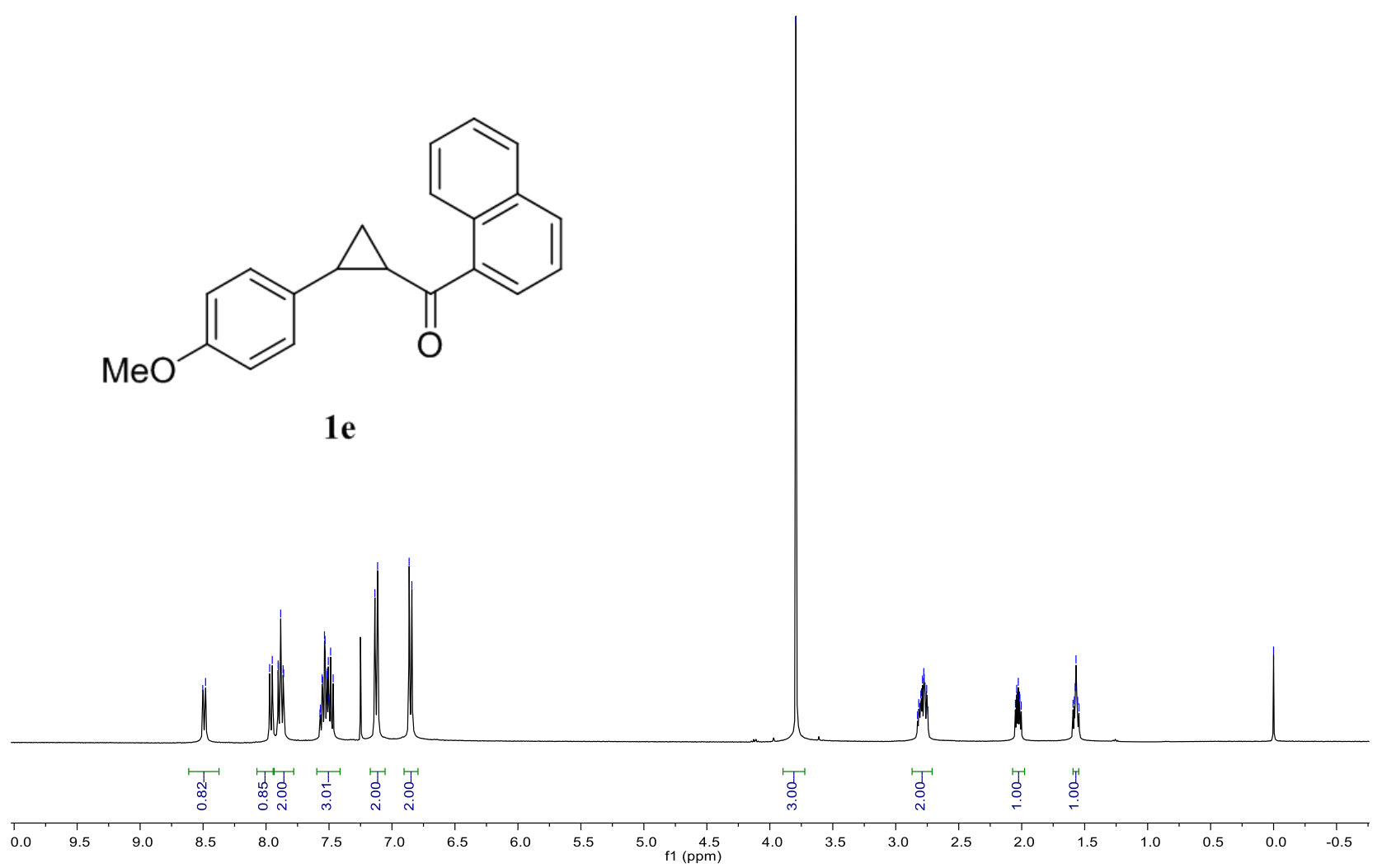

离

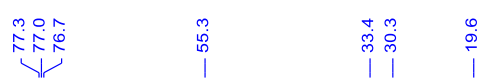

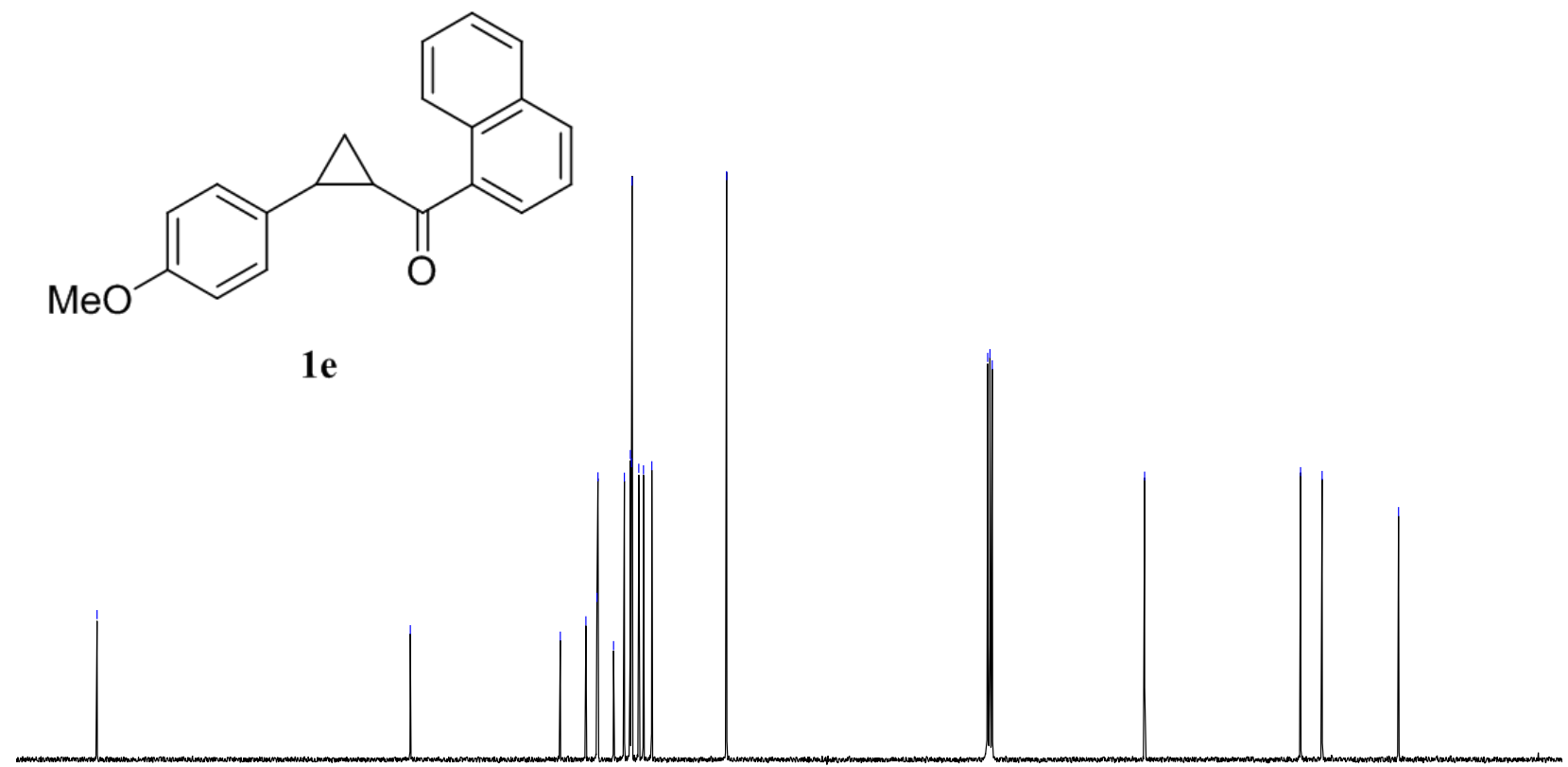

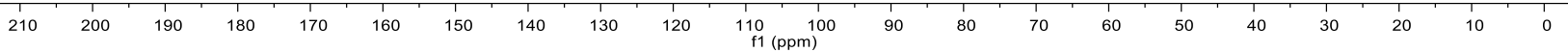


<smiles>COc1ccc(C2CC2C(=O)c2ccc(F)cc2)cc1</smiles>

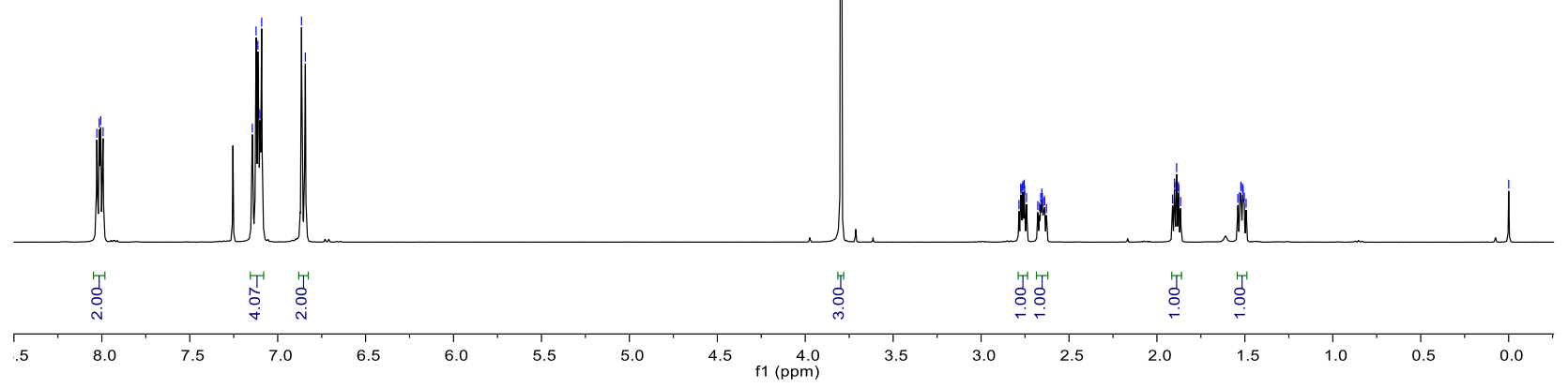

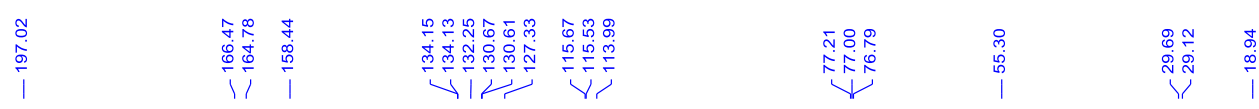
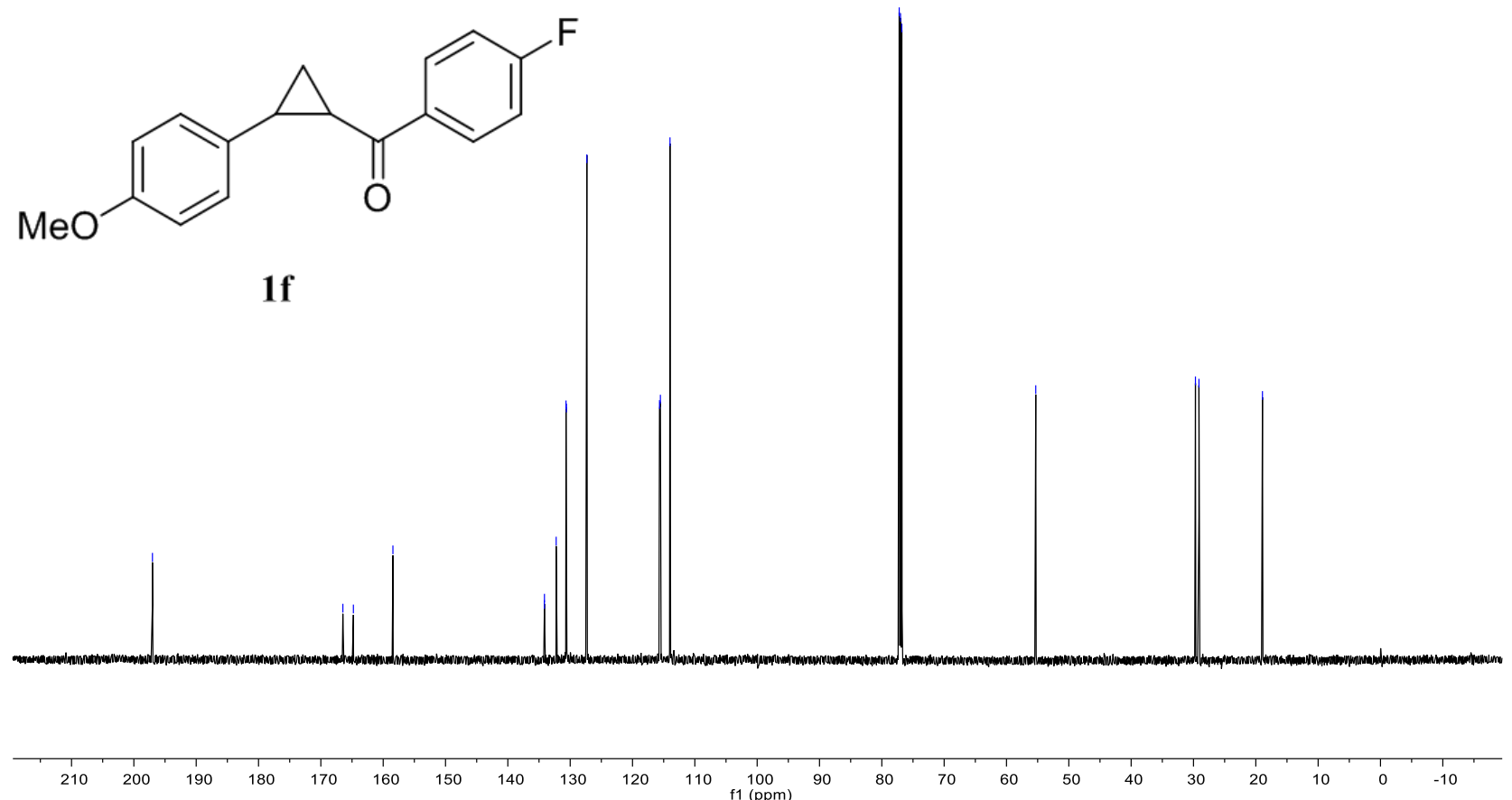

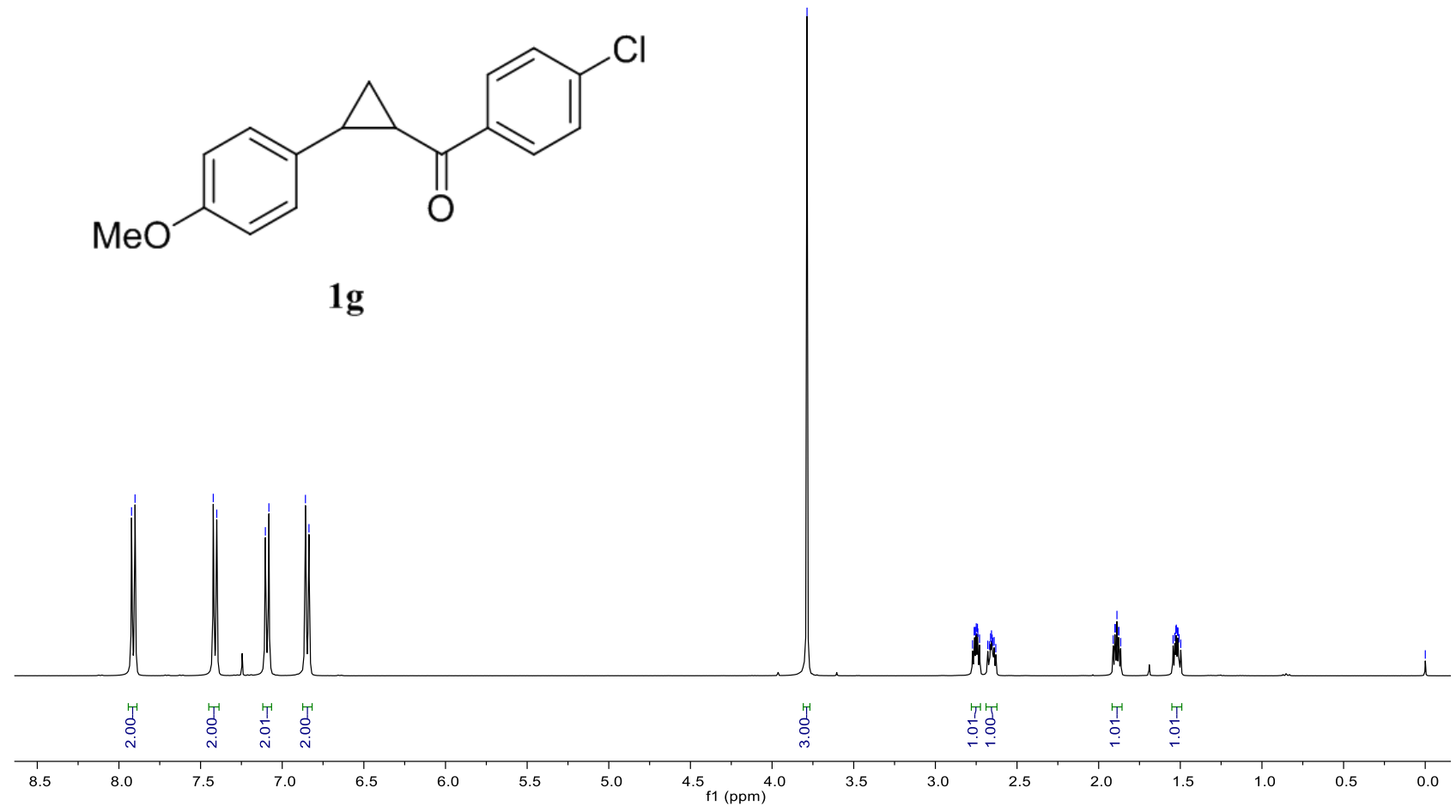

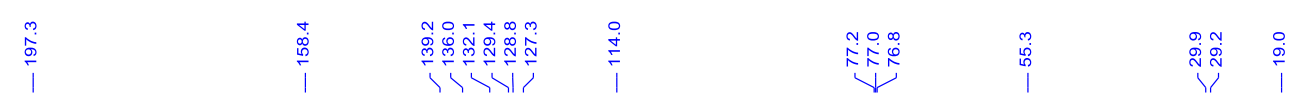

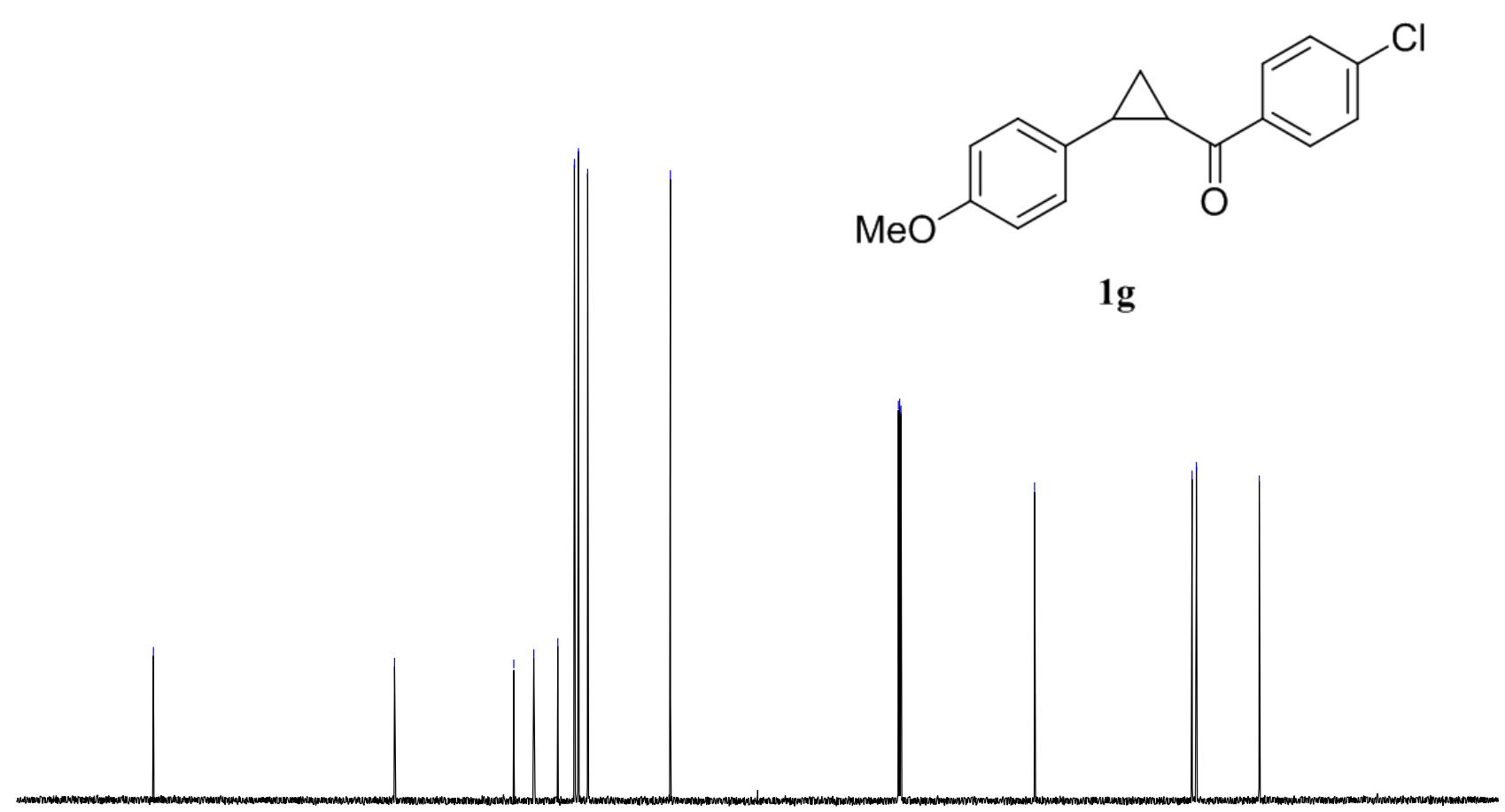

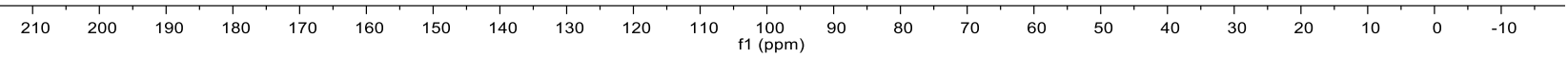




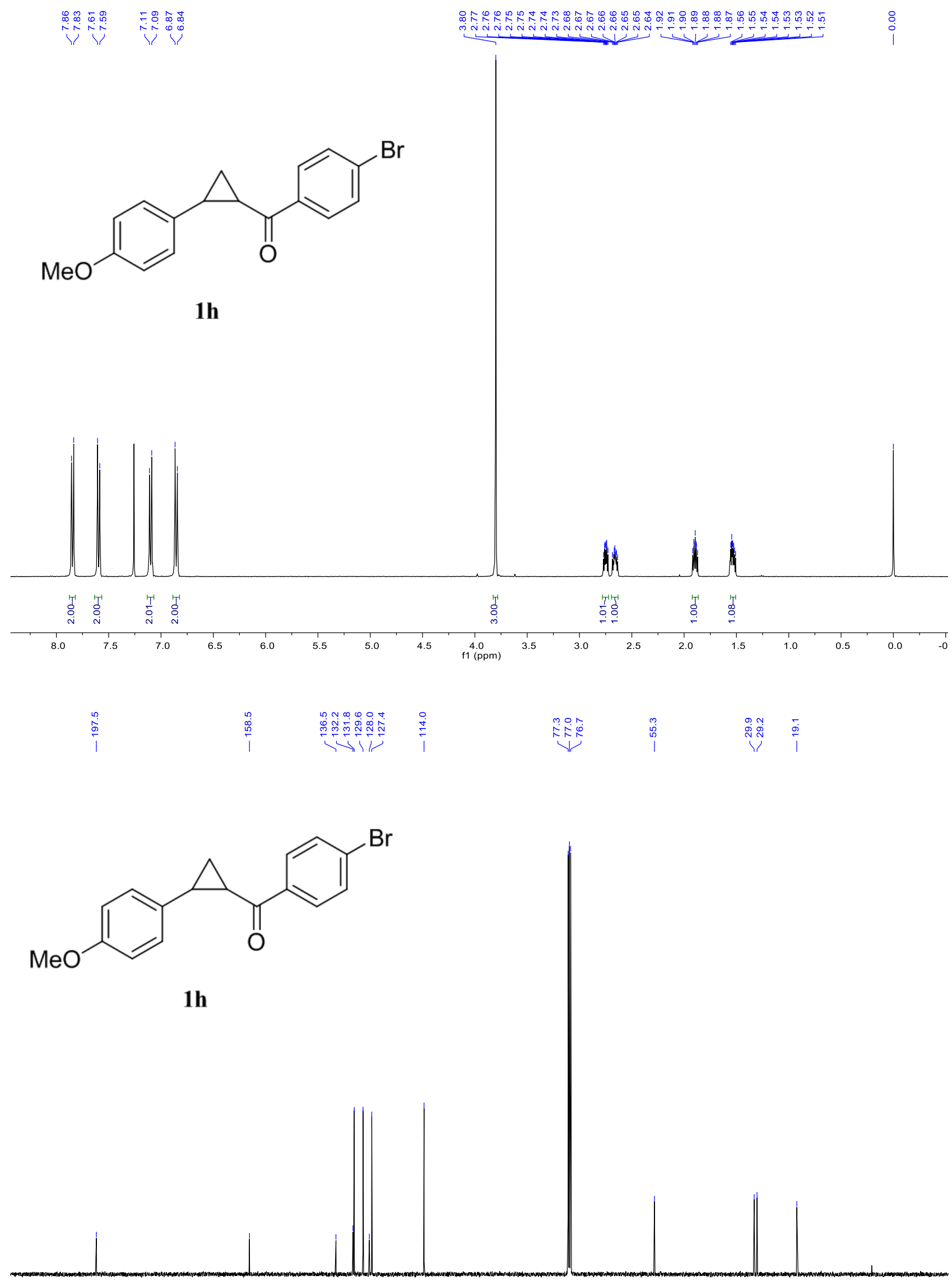

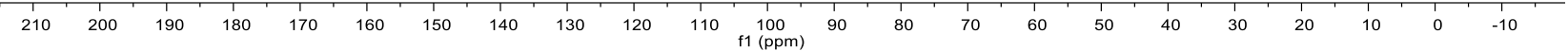


<smiles>COc1ccc(C2CC2C(=O)c2ccc(C(F)(F)F)cc2)cc1</smiles>

1i
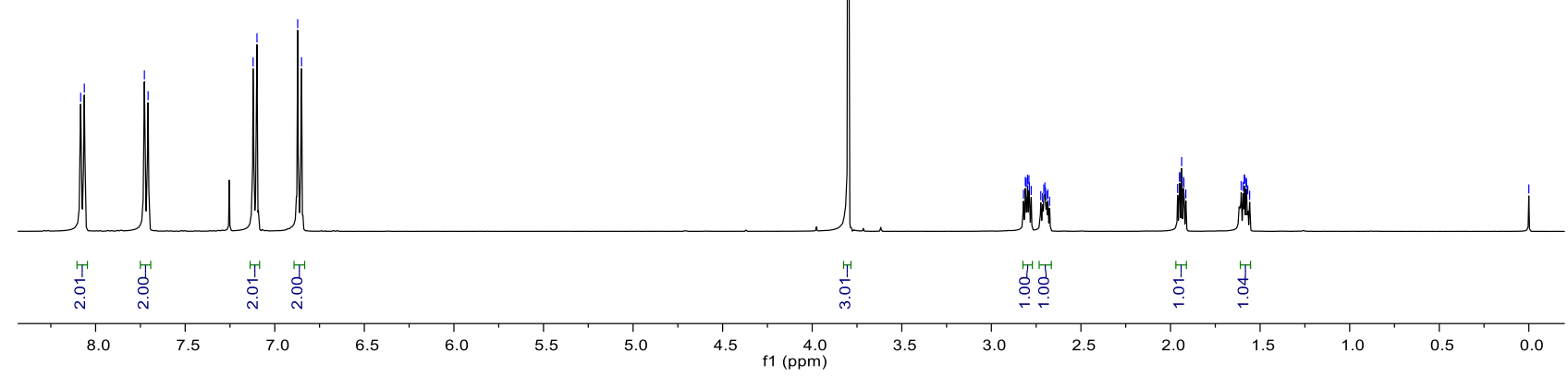

|

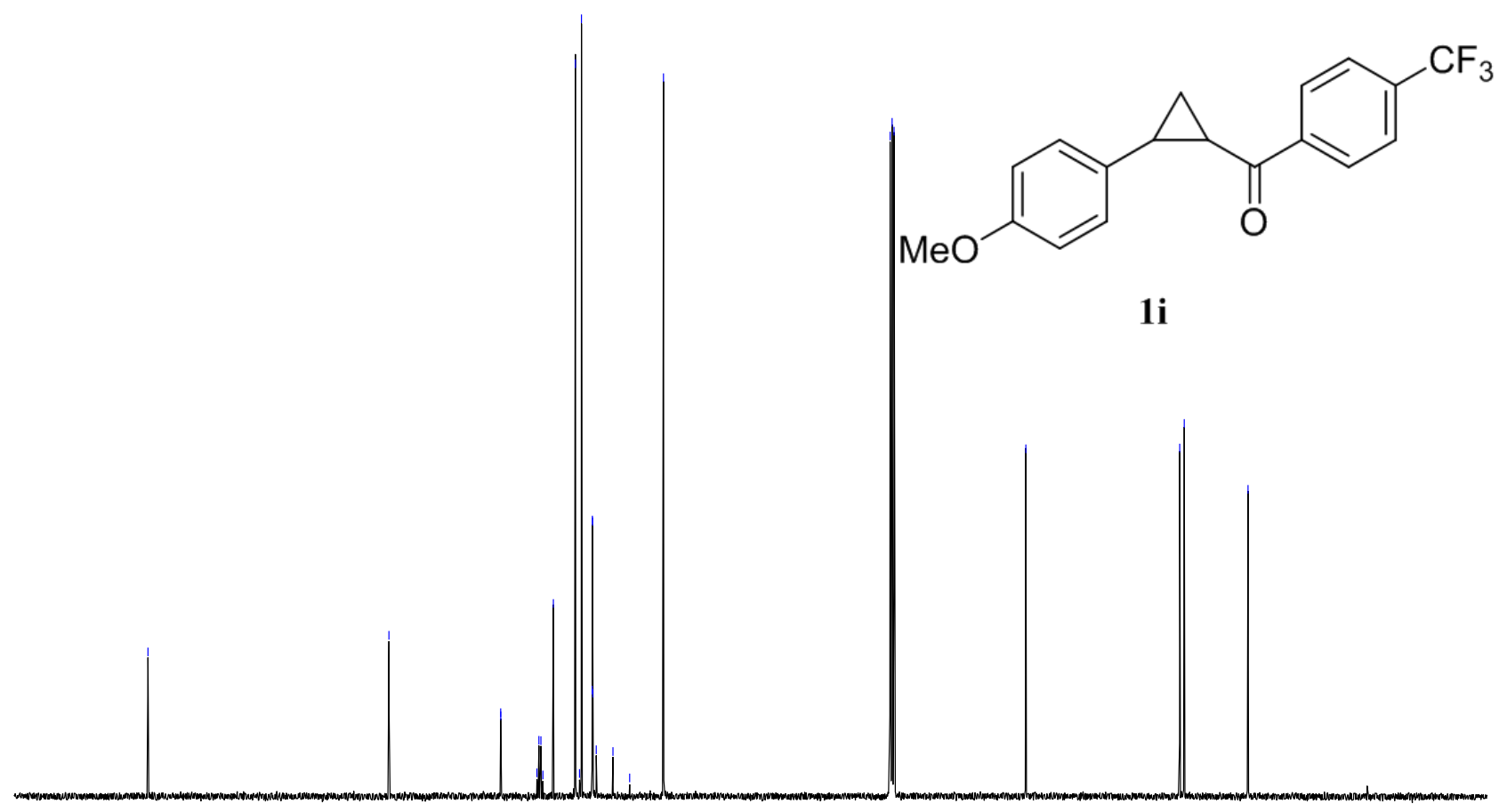

$\begin{array}{llllllllllll}210 & 200 & 190 & 180 & 170 & 160 & 150 & 140 & 130 & 120 & 110 & \begin{array}{c}100 \\ \mathrm{f} 1(\mathrm{ppm})\end{array}\end{array}$ 

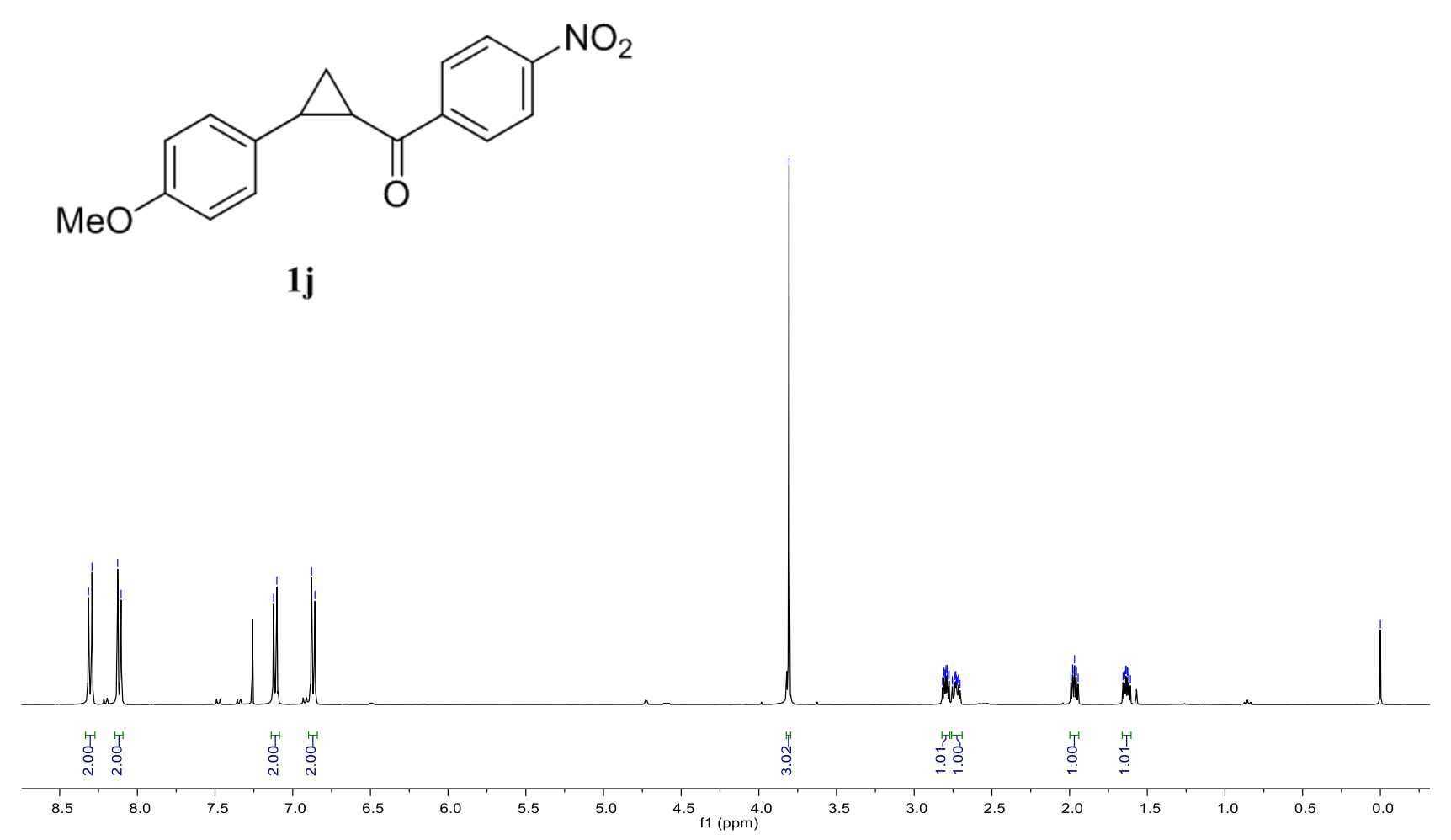

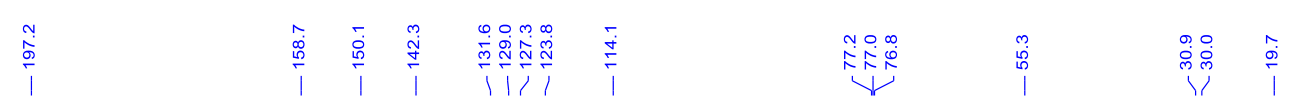

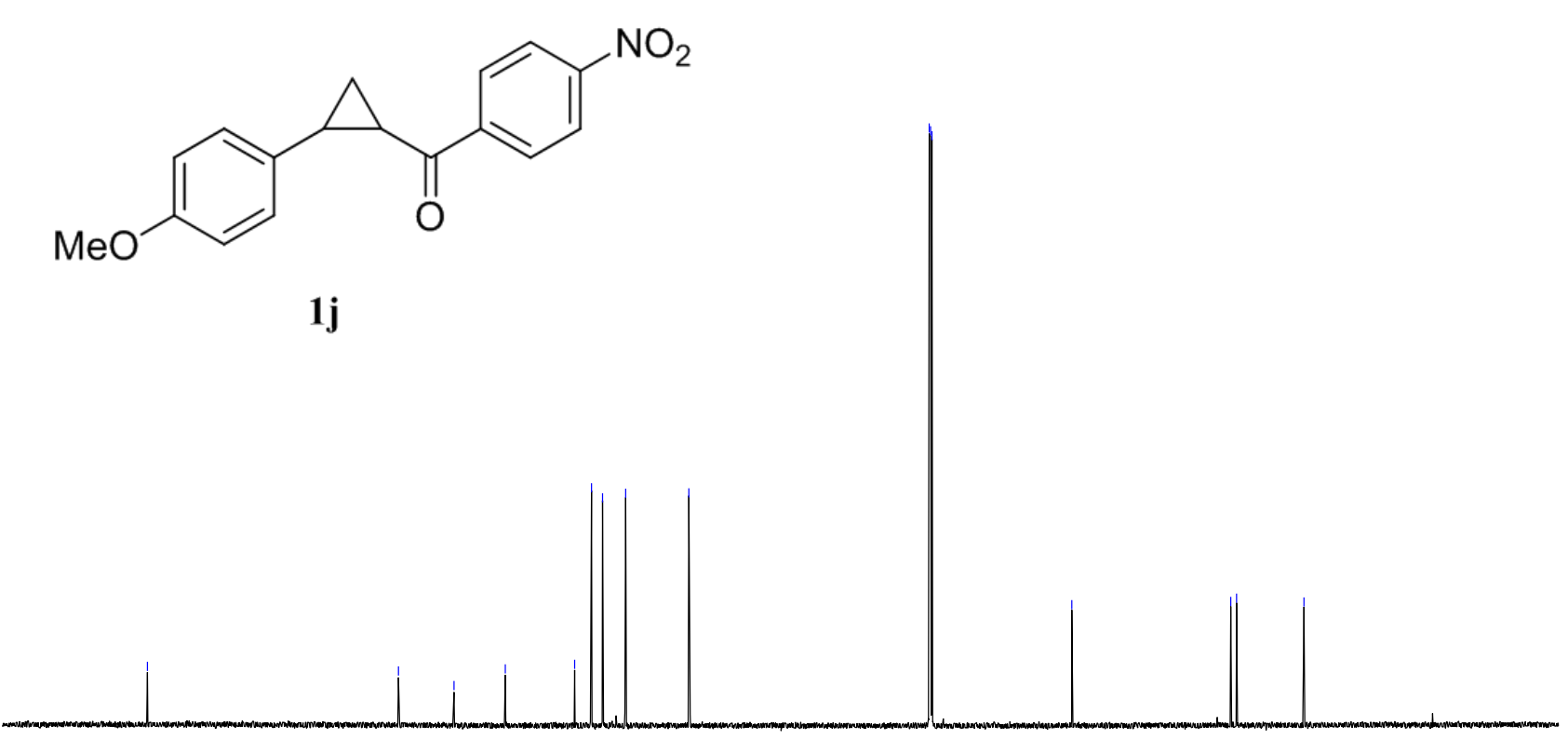

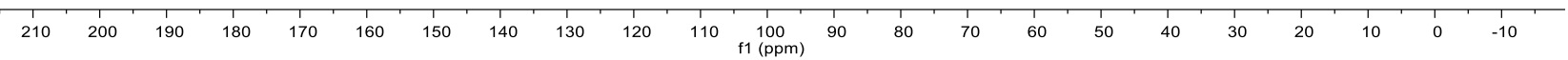


<smiles>COc1ccc(C2CC2C(=O)c2cccc(OC)c2)cc1</smiles>

$1 \mathrm{k}$
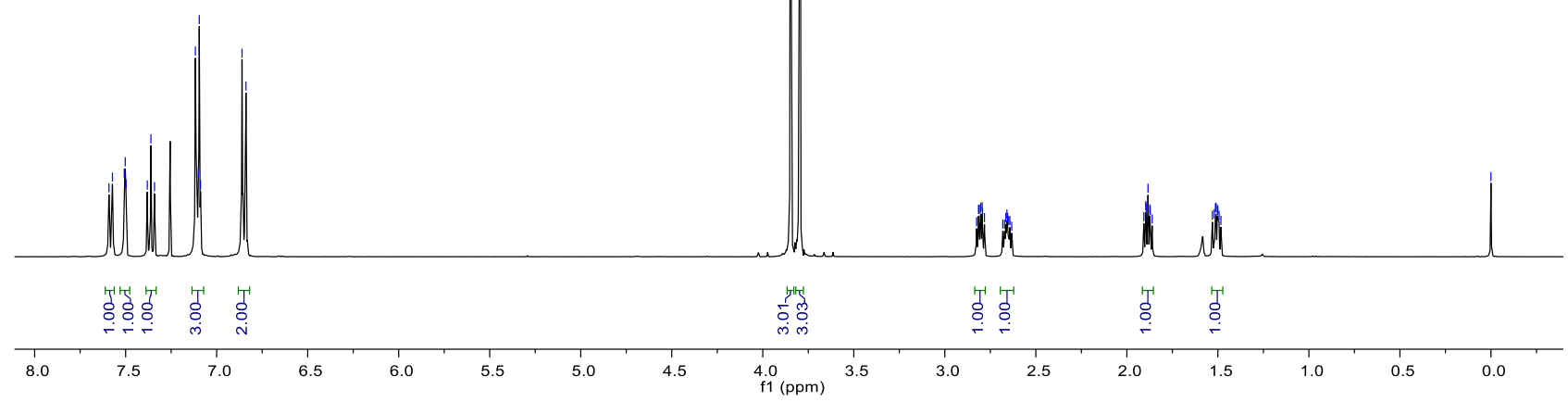

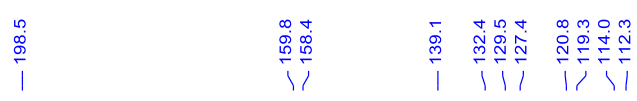

Vy

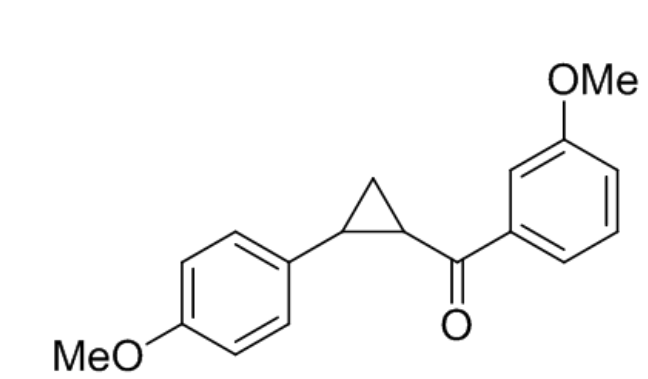

$1 \mathrm{k}$

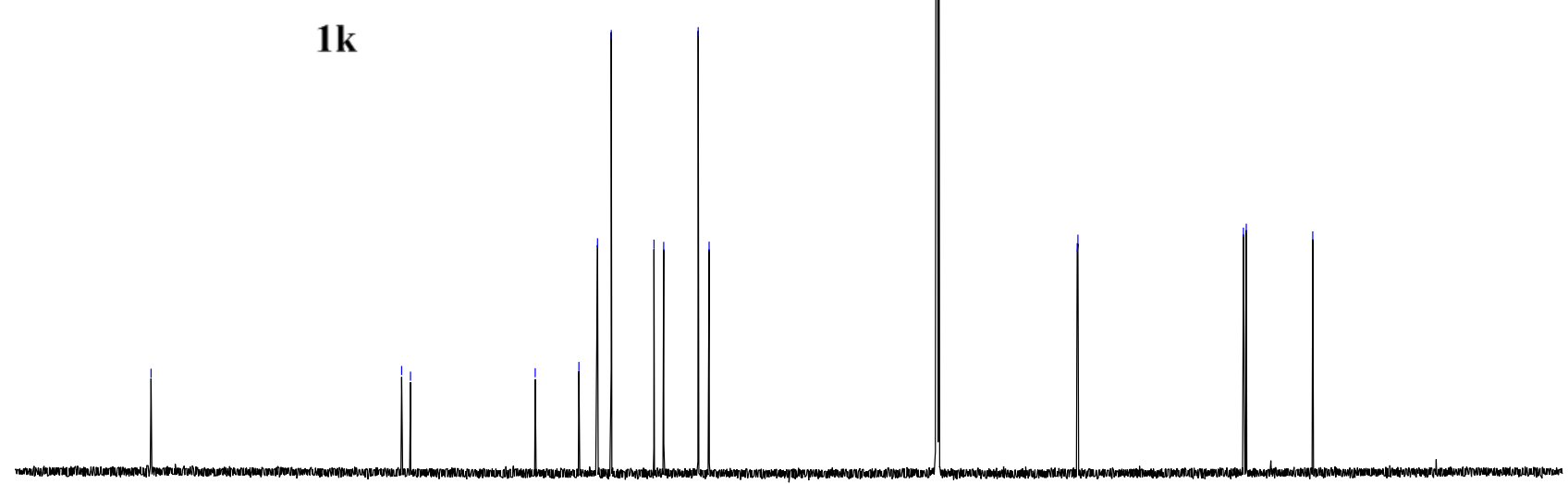

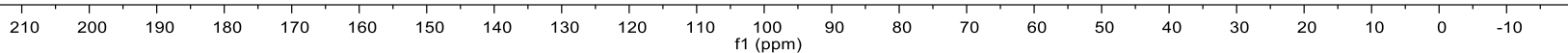


<smiles>COc1ccc(C2CC2C(=O)c2cccc(Cl)c2)cc1</smiles>

11
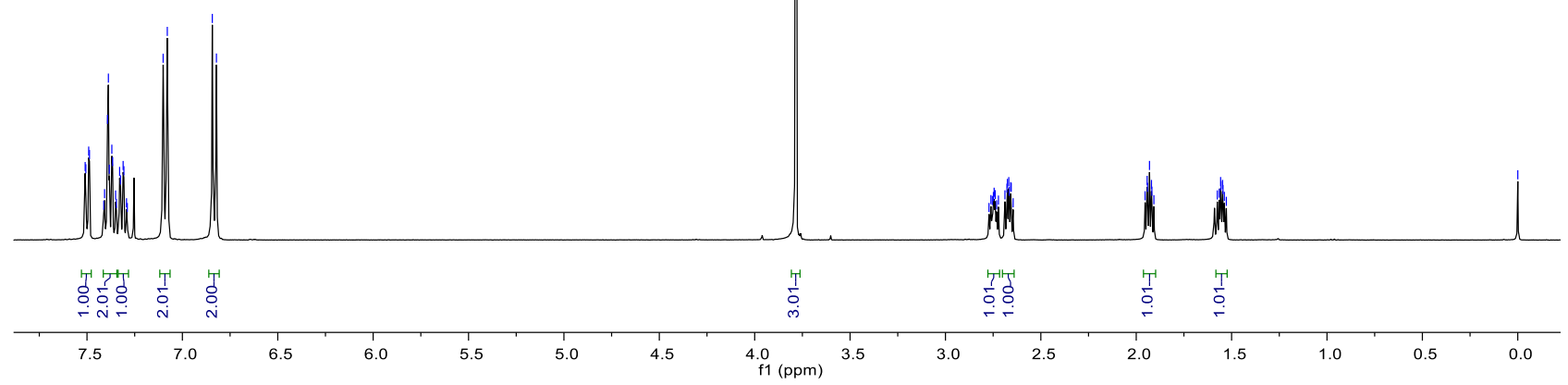

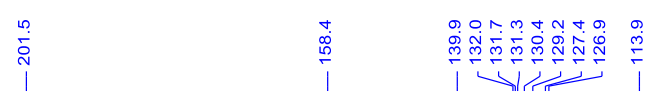

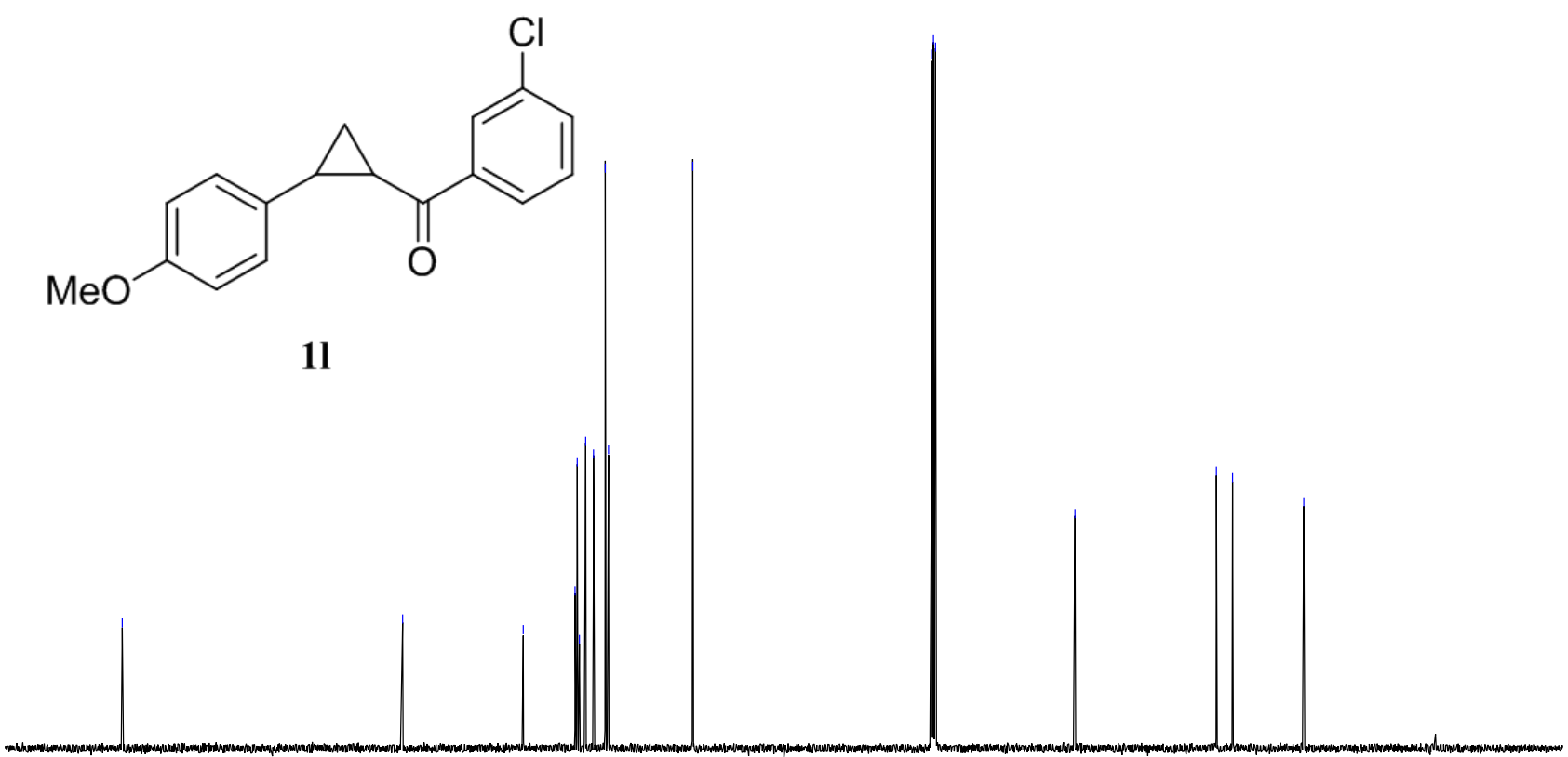

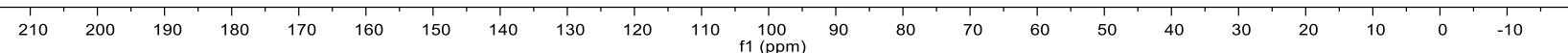


<smiles>COc1ccccc1C1CC1C(=O)c1ccccc1</smiles>

$1 \mathrm{~m}$

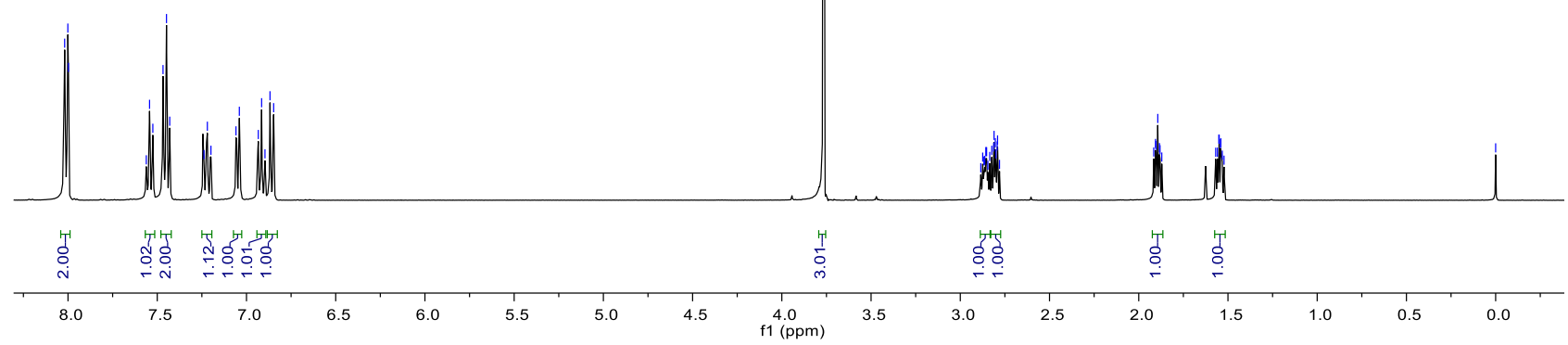

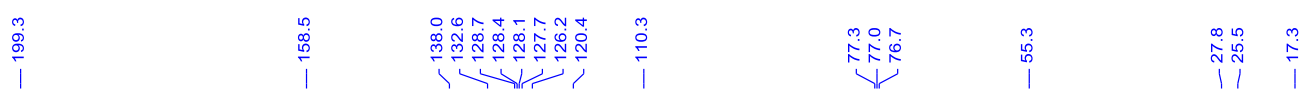

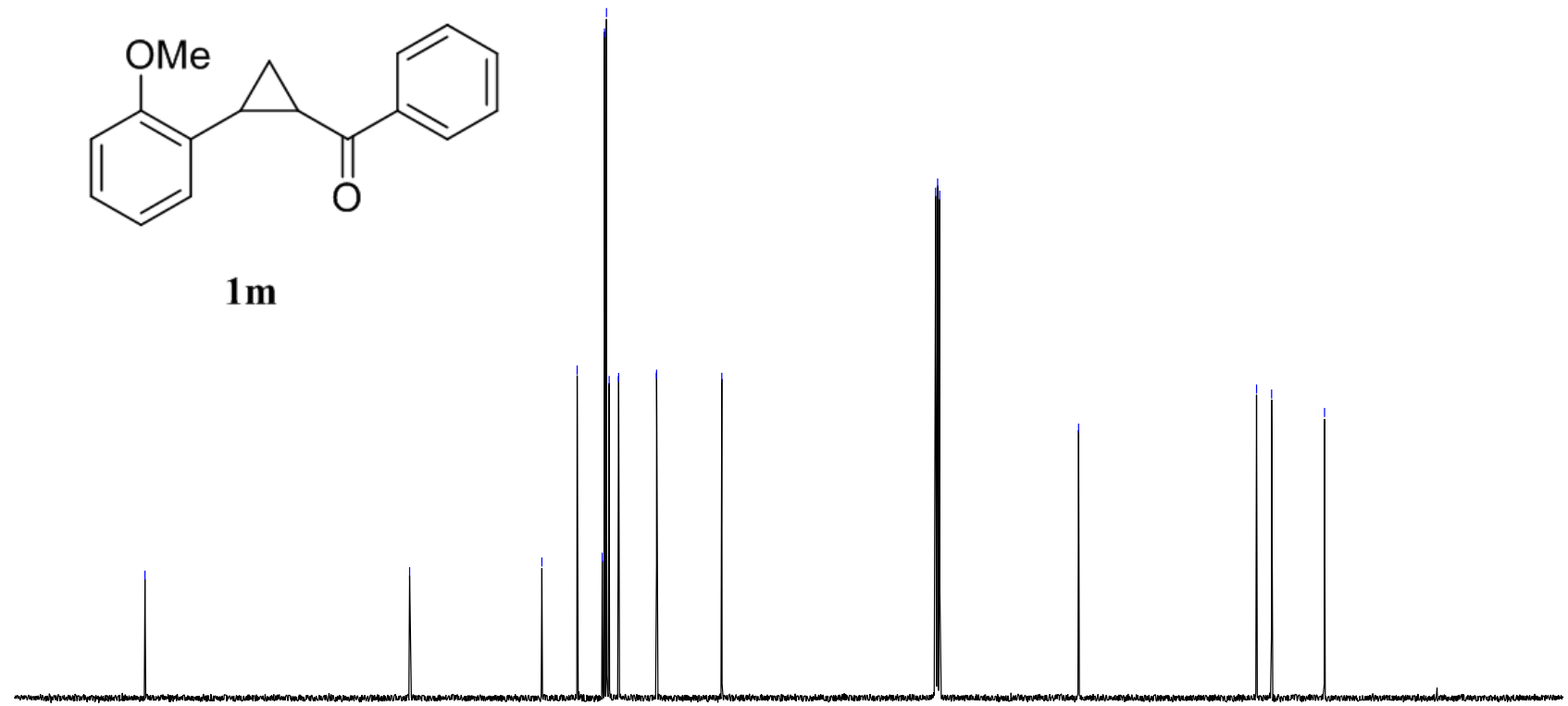

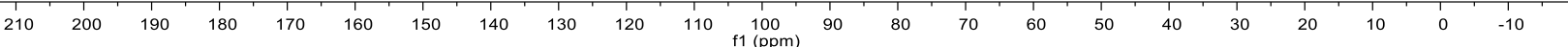




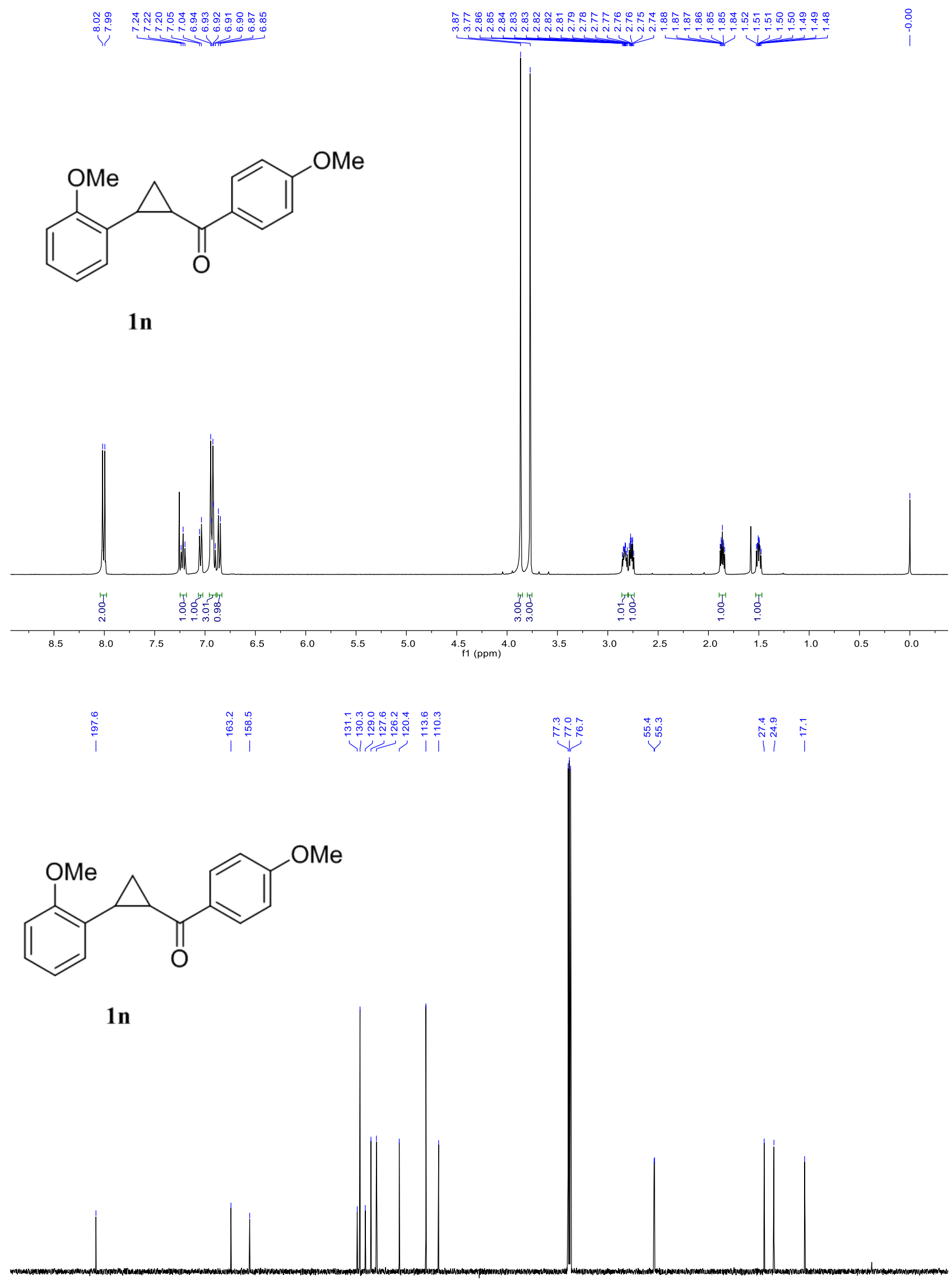

$\underbrace{}_{8.5}$

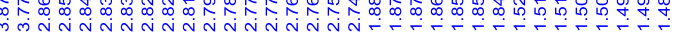


<smiles>COc1ccccc1C1CC1C(=O)c1ccc(F)cc1</smiles>

10

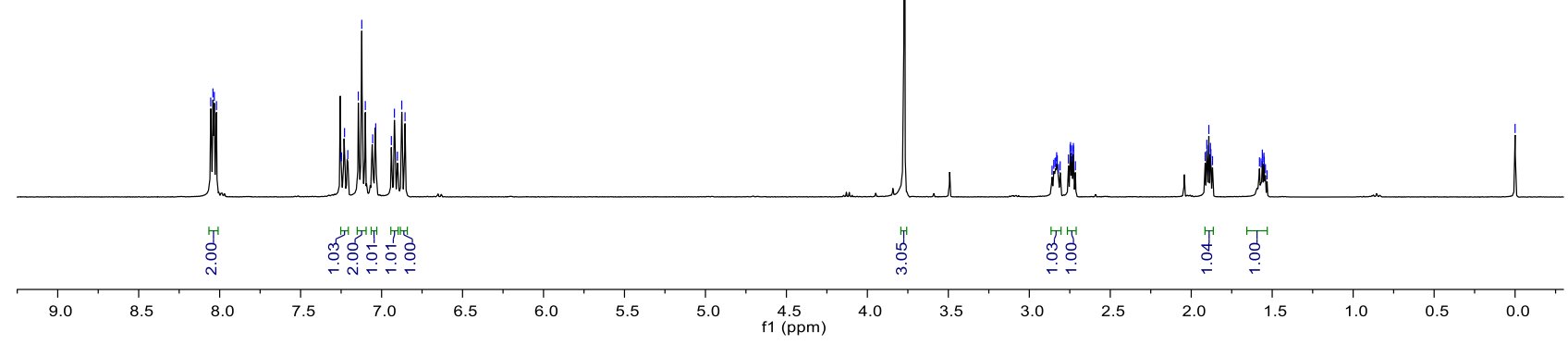

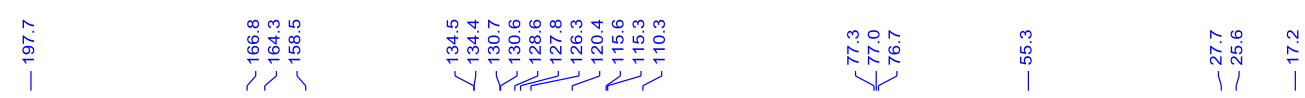<smiles>COc1ccccc1C1CC1C(=O)c1ccc(F)cc1</smiles>

10

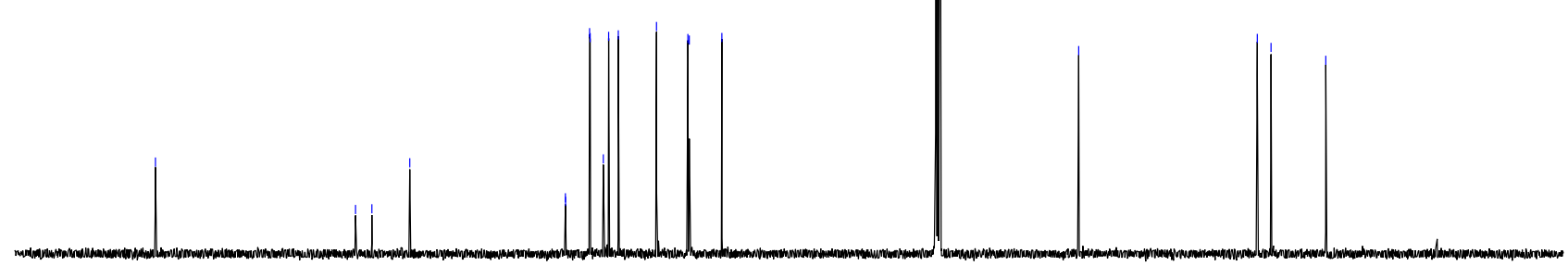

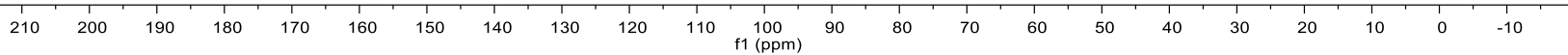


<smiles>COc1ccccc1C1CC1C(=O)c1ccc(C(F)(F)F)cc1</smiles>

$1 p$

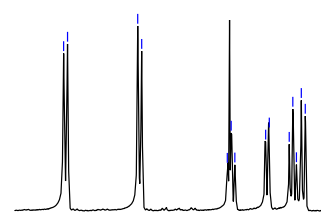

A

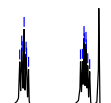

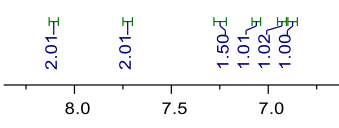

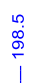<smiles>COc1ccccc1C1CC1C(=O)c1ccc(C(F)(F)F)cc1</smiles>

$1 p$

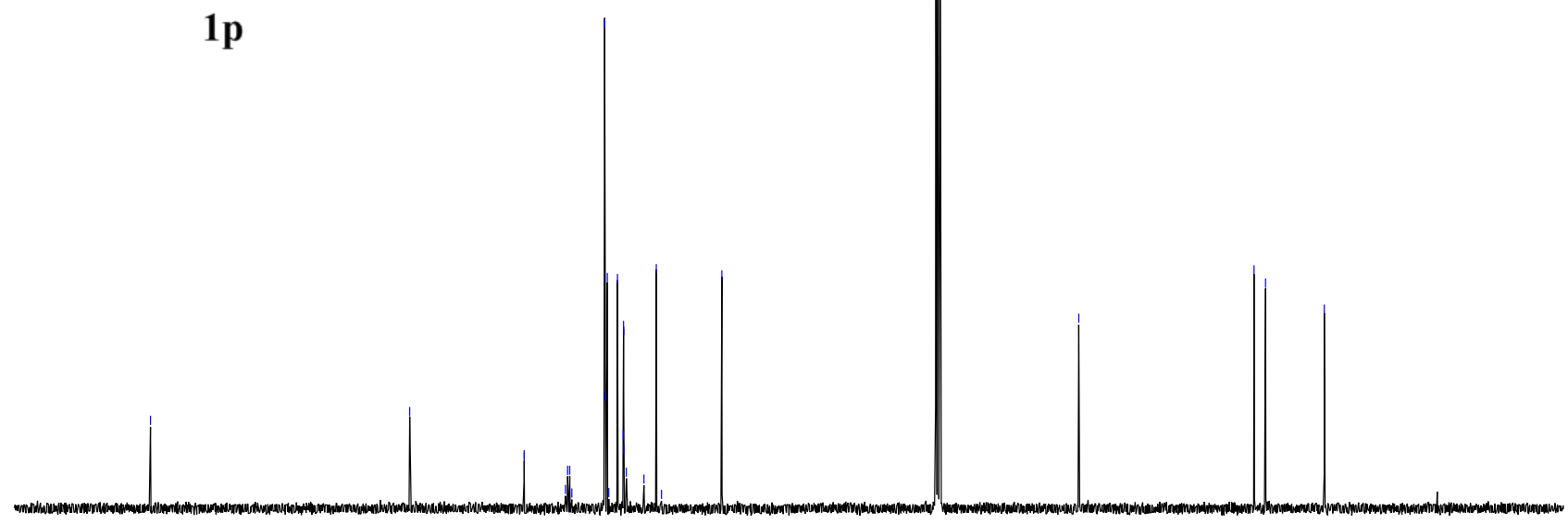

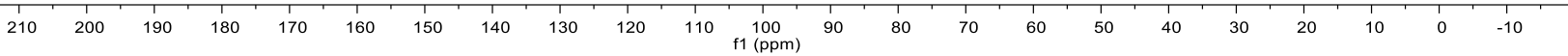




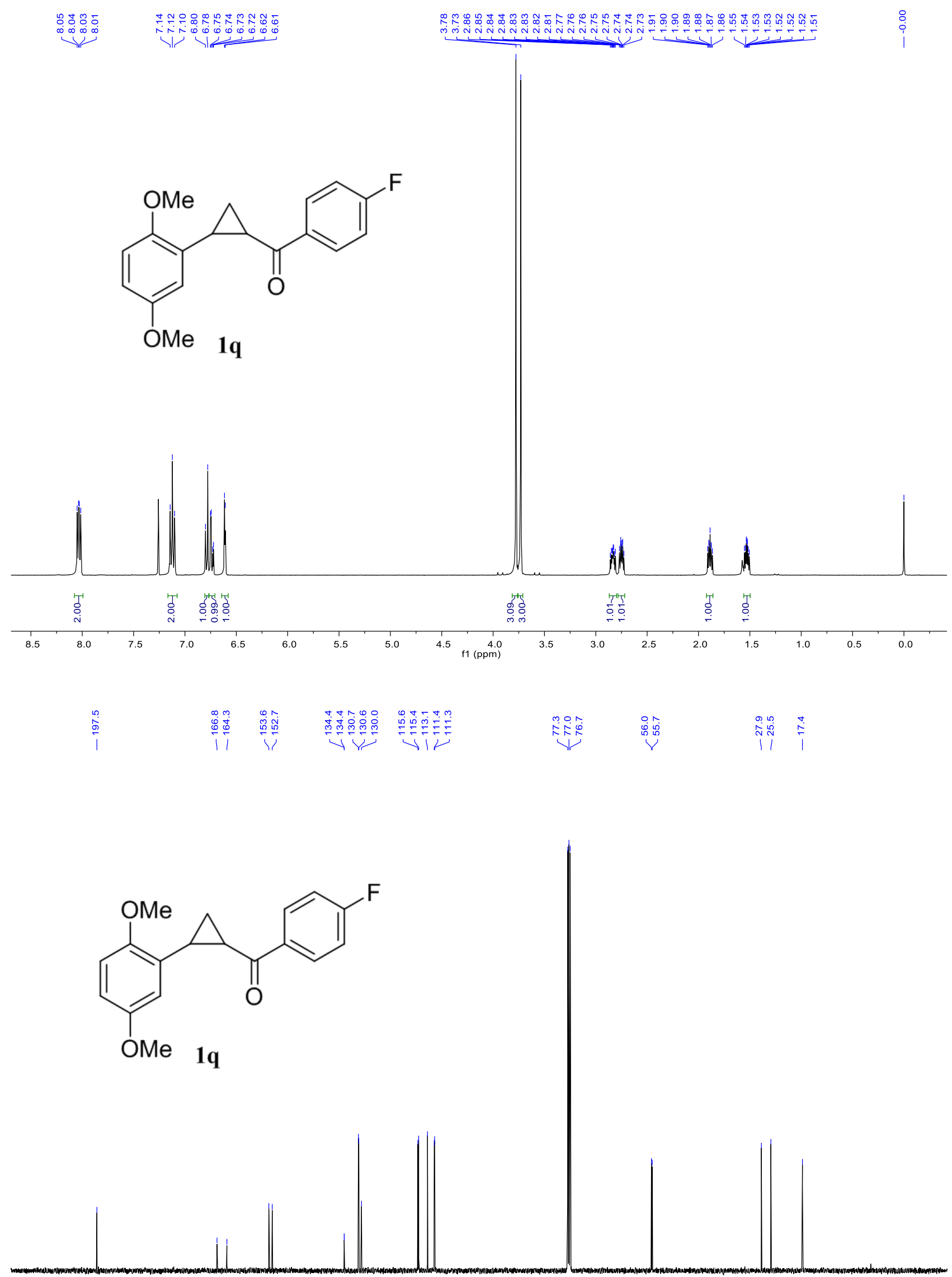

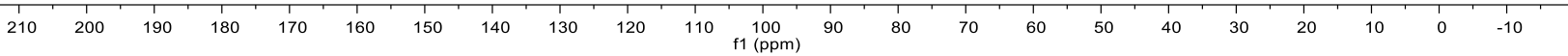


<smiles>COc1ccc(OC)c(C2CC2C(=O)c2ccc(C(F)(F)F)cc2)c1</smiles>
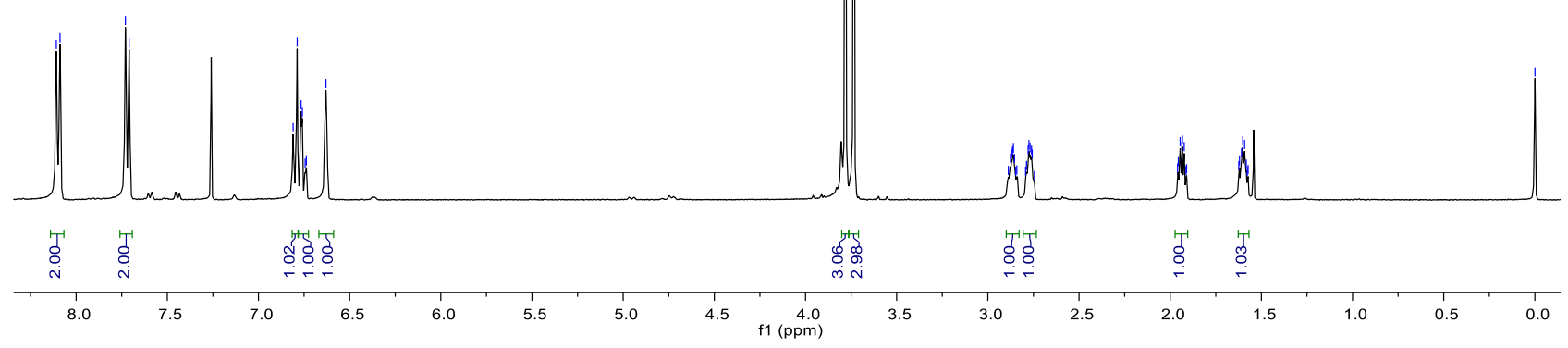

i<smiles>COc1ccc(OC)c(C2CC2C(=O)c2ccc(C(F)(F)F)cc2)c1</smiles>

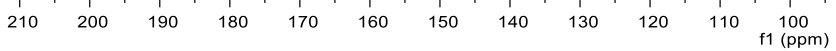



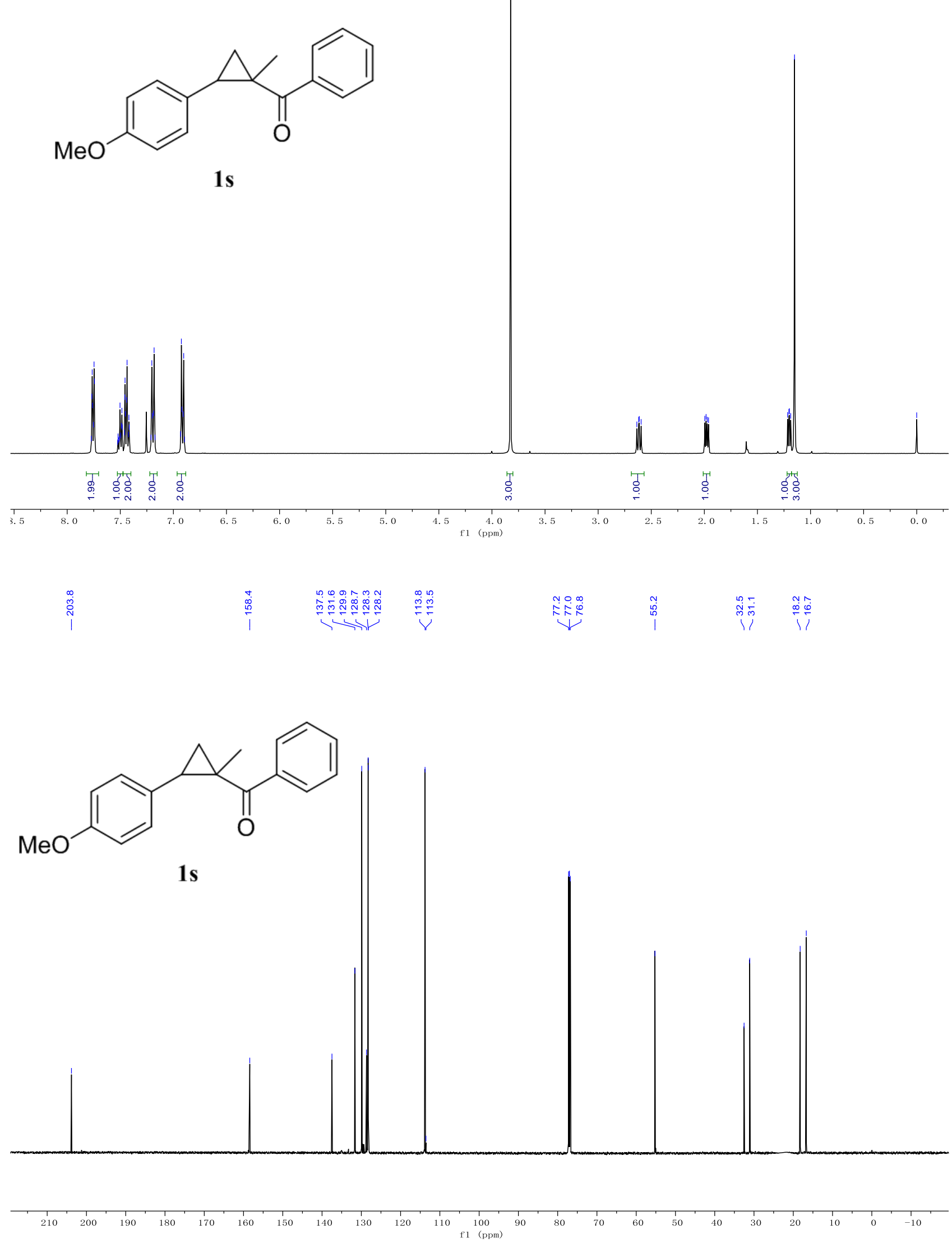

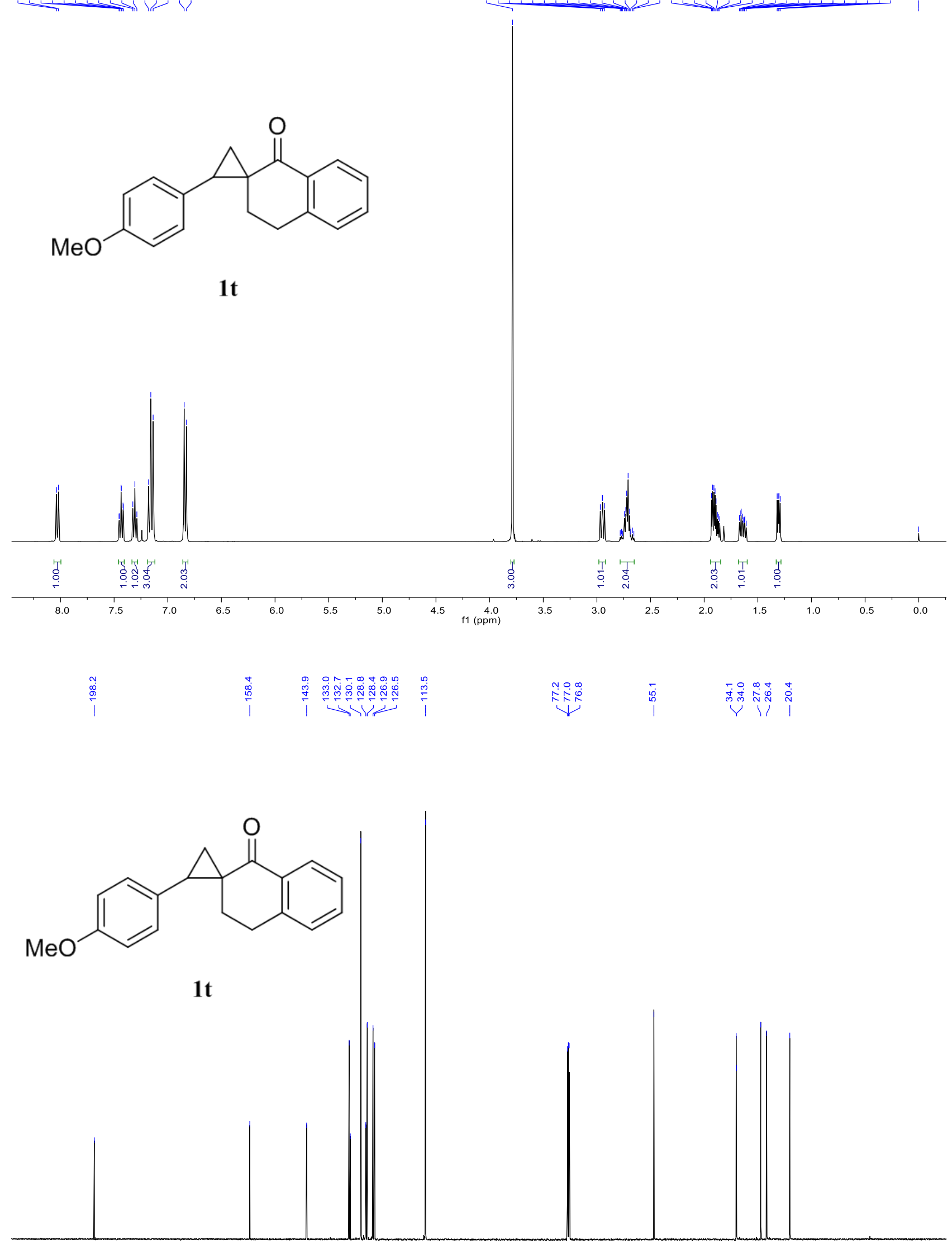

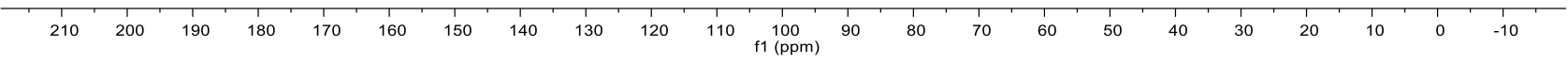




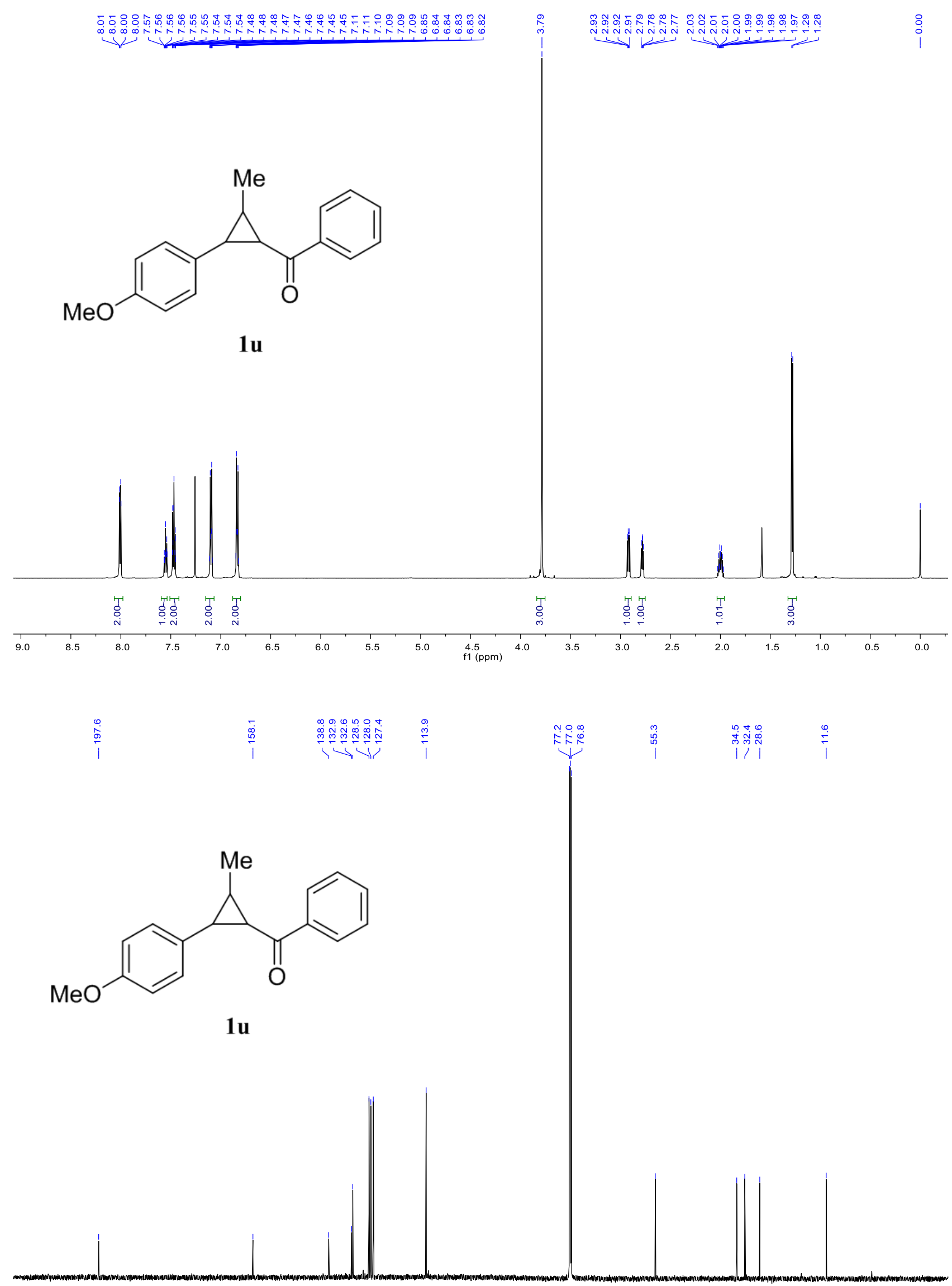

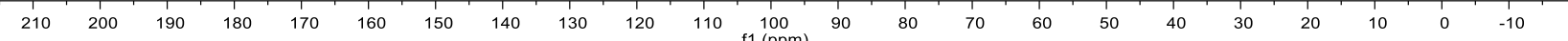




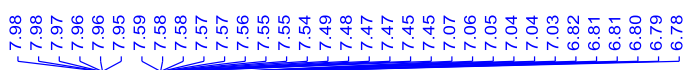

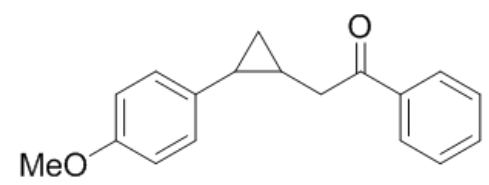

1w

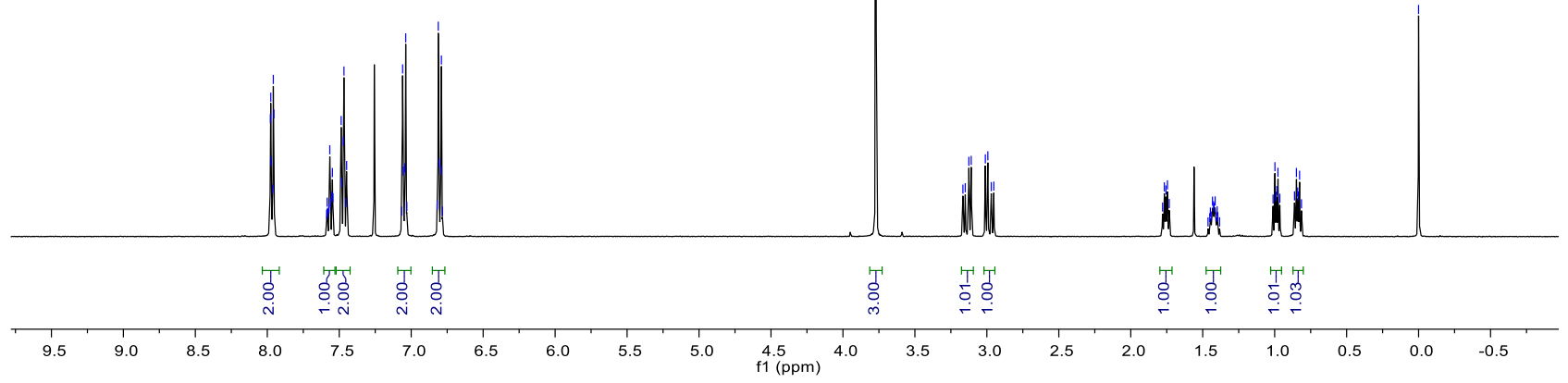

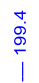

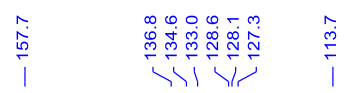

管
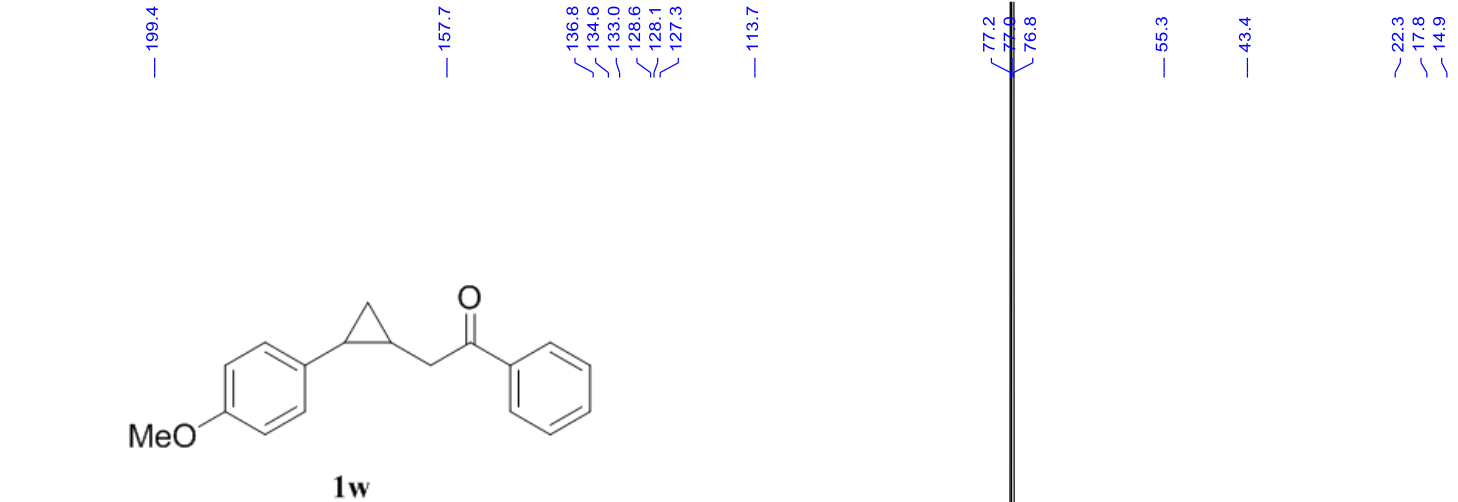

$1 w$

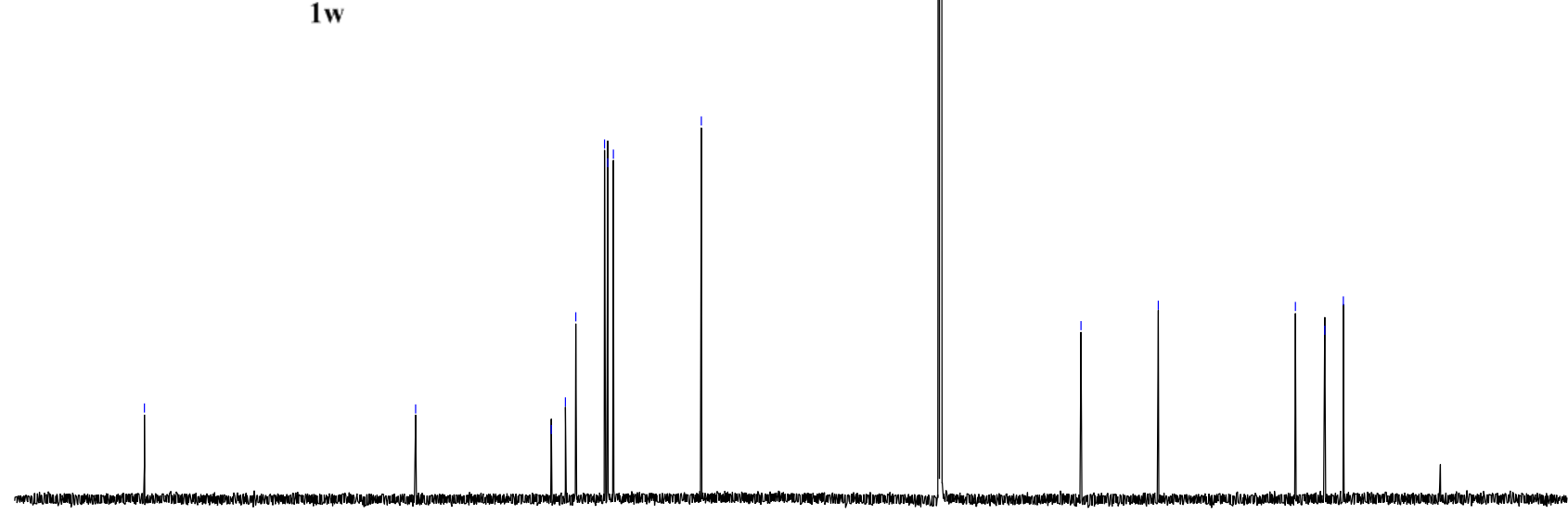

$\underbrace{-10}$

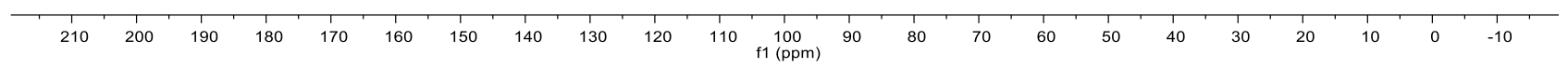

S28 

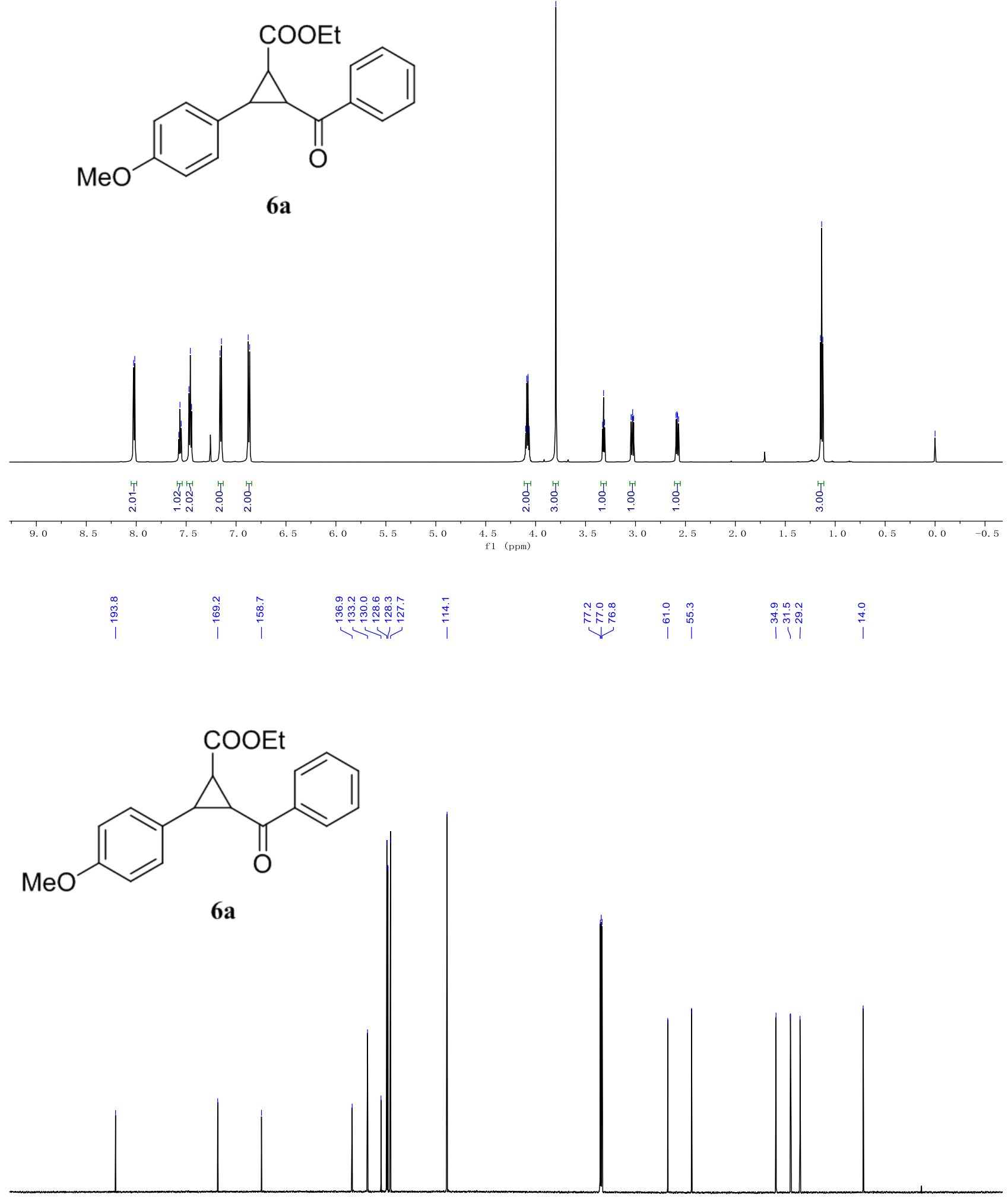

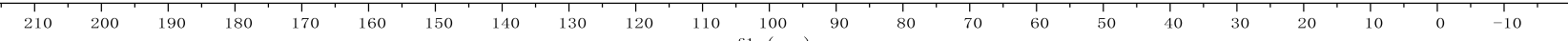




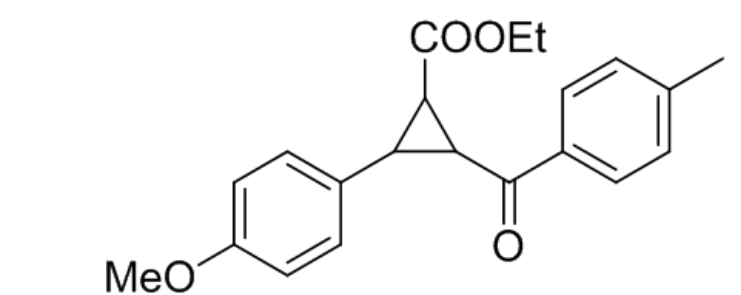

6b

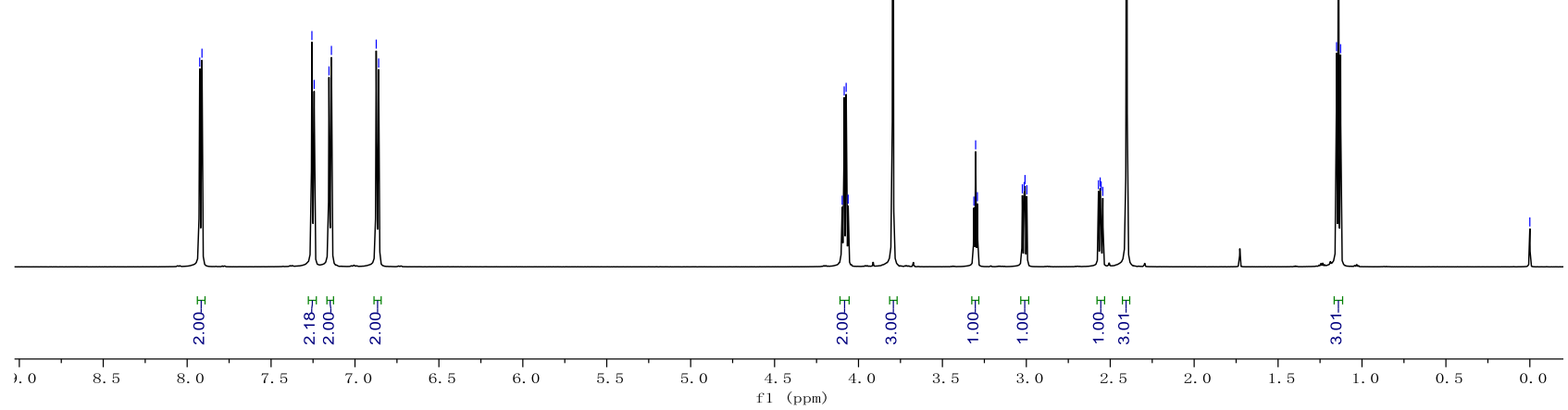

每<smiles>CCOC(=O)C1C(C(=O)c2ccc(C)cc2)C1c1ccc(OC)cc1</smiles>

6b
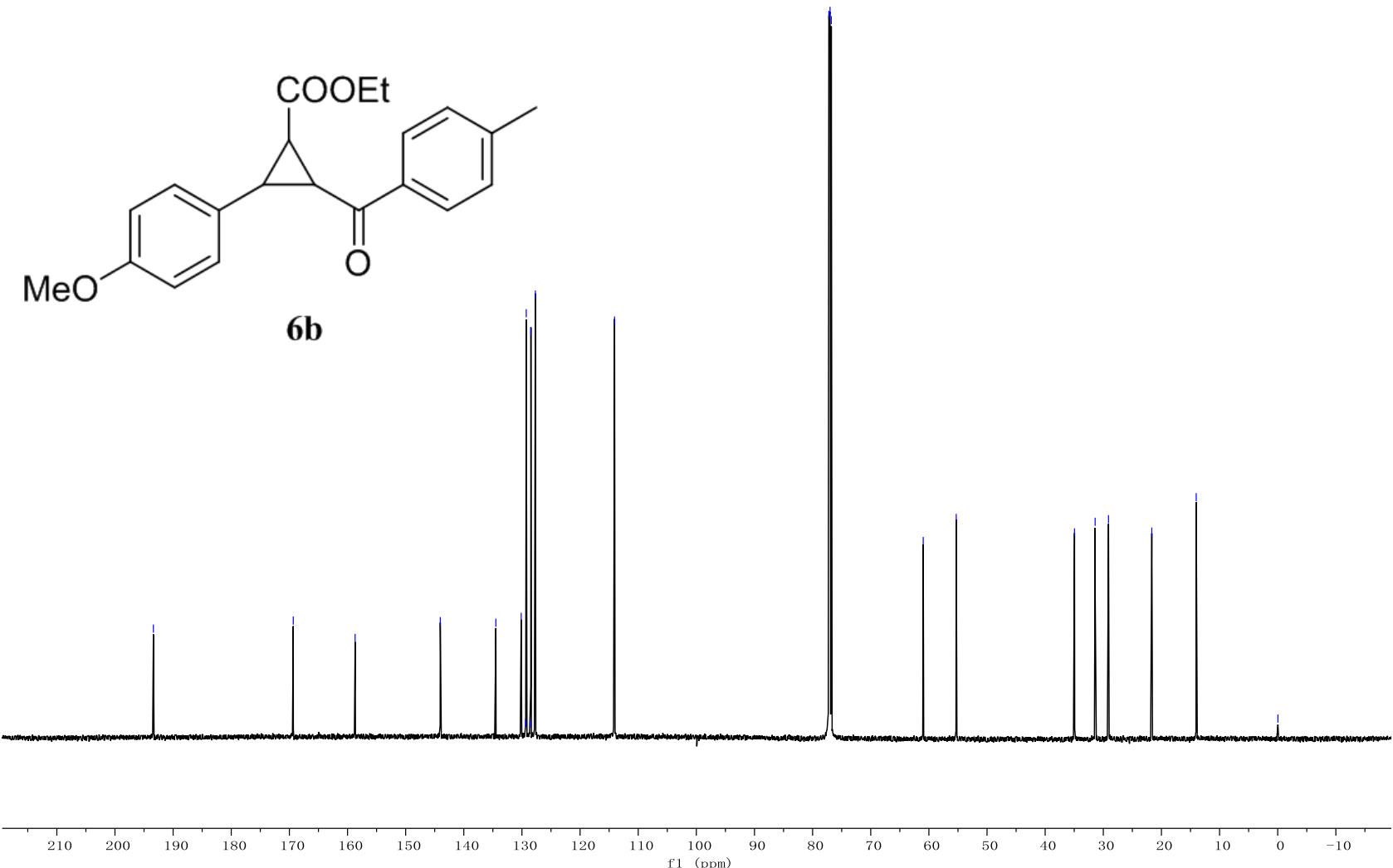


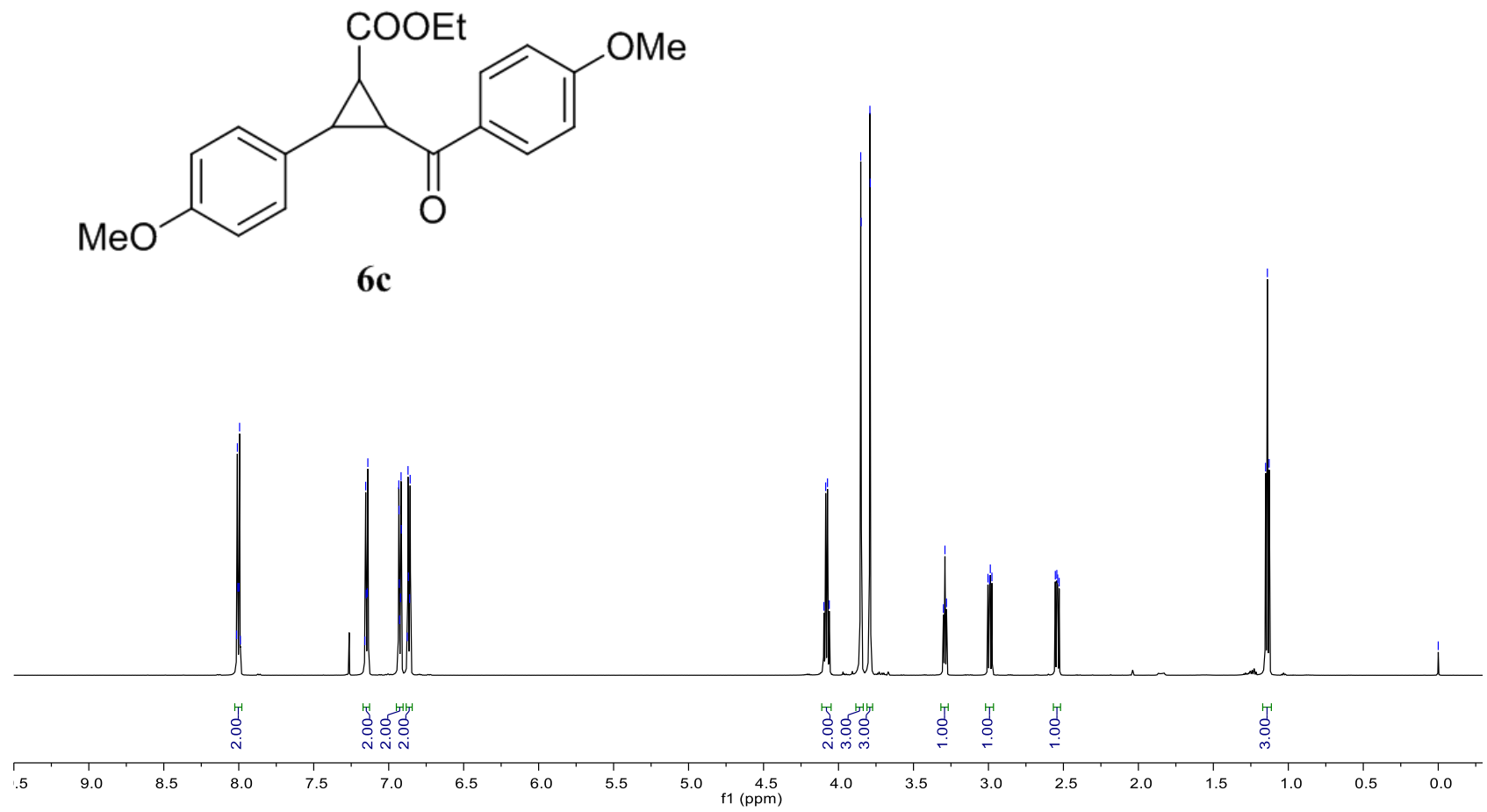

每<smiles>CCOC(=O)C1C(C(=O)c2ccc(OC)cc2)C1c1ccc(OC)cc1</smiles>

6c

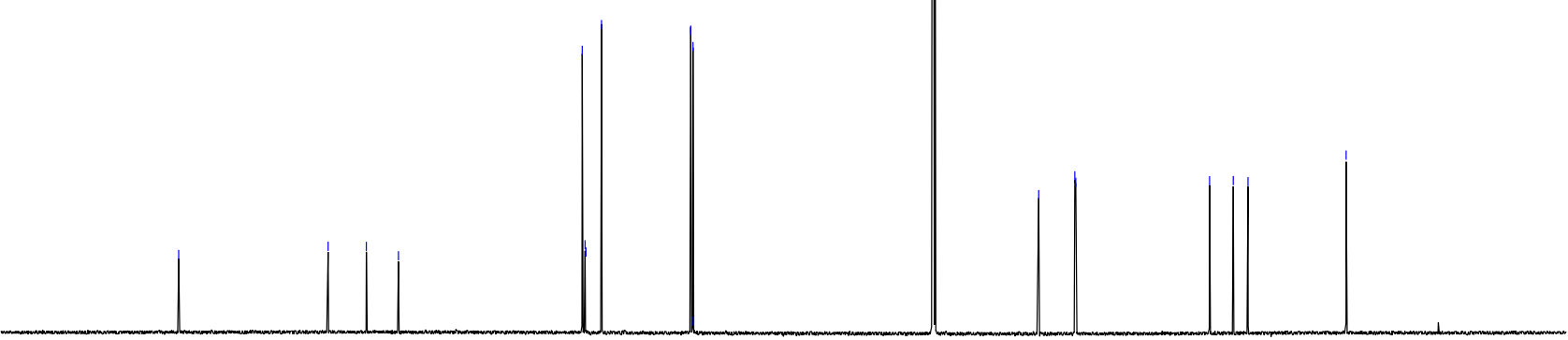

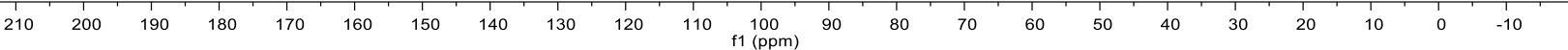




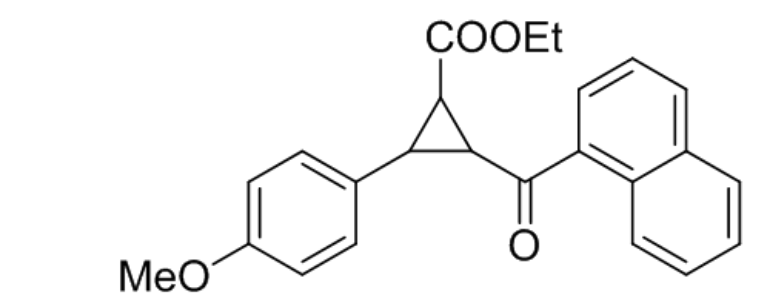

6d

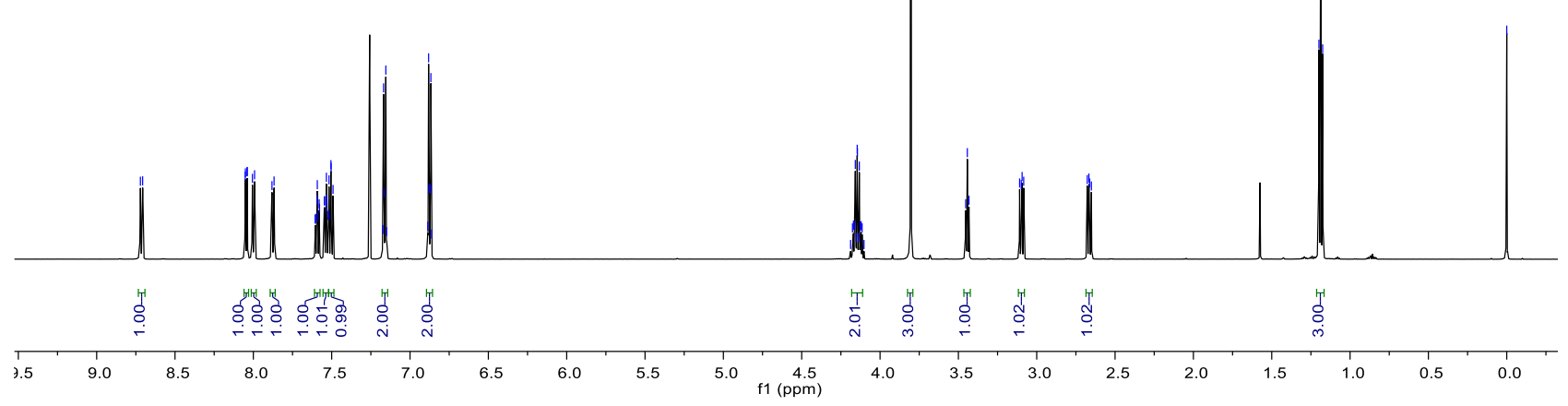

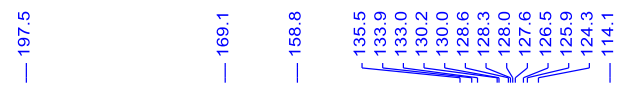

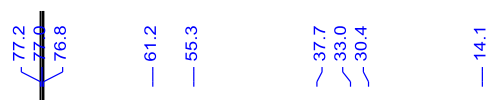<smiles>CCOC(=O)C1C(C(=O)c2cccc3ccccc23)C1c1ccc(OC)cc1</smiles>

6d

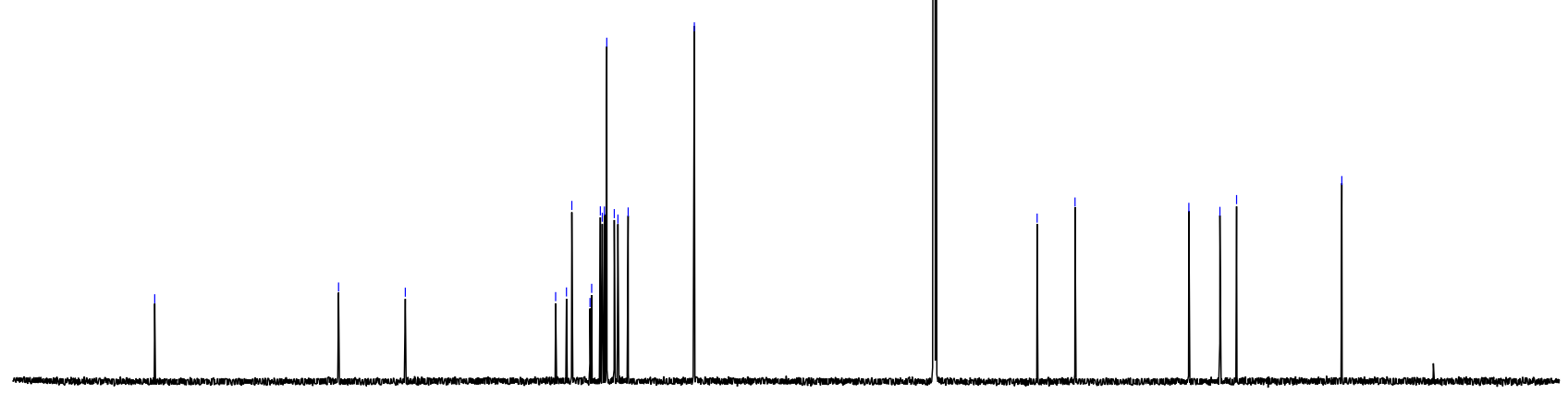

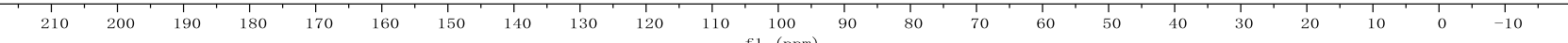




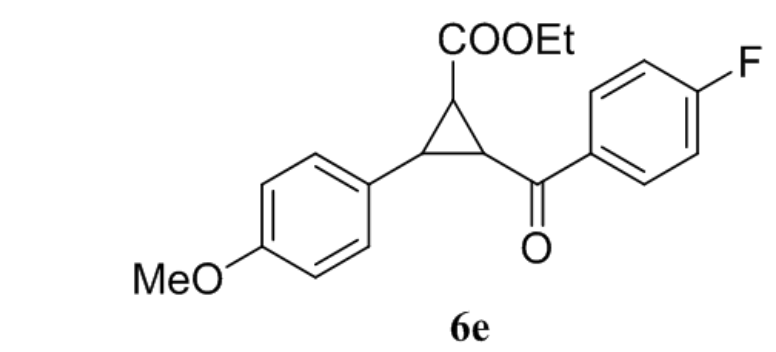

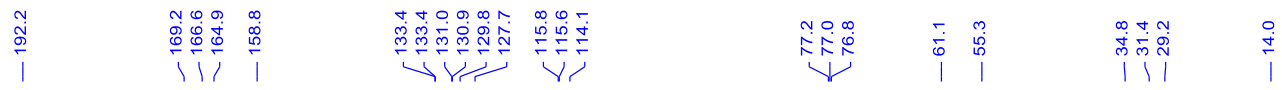<smiles>CCOC(=O)C1C(C(=O)c2ccc(F)cc2)C1c1ccc(OC)cc1</smiles>

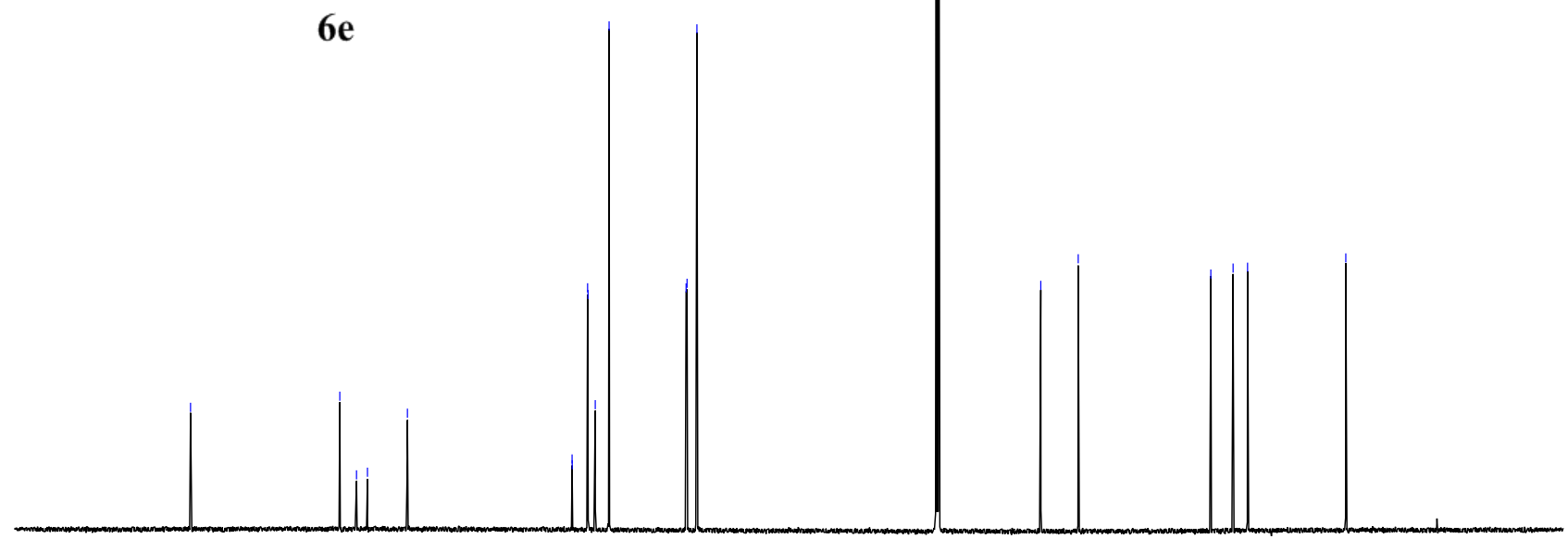

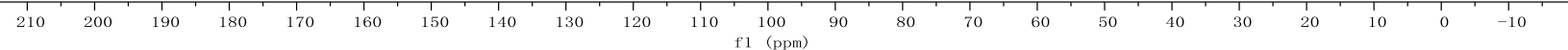




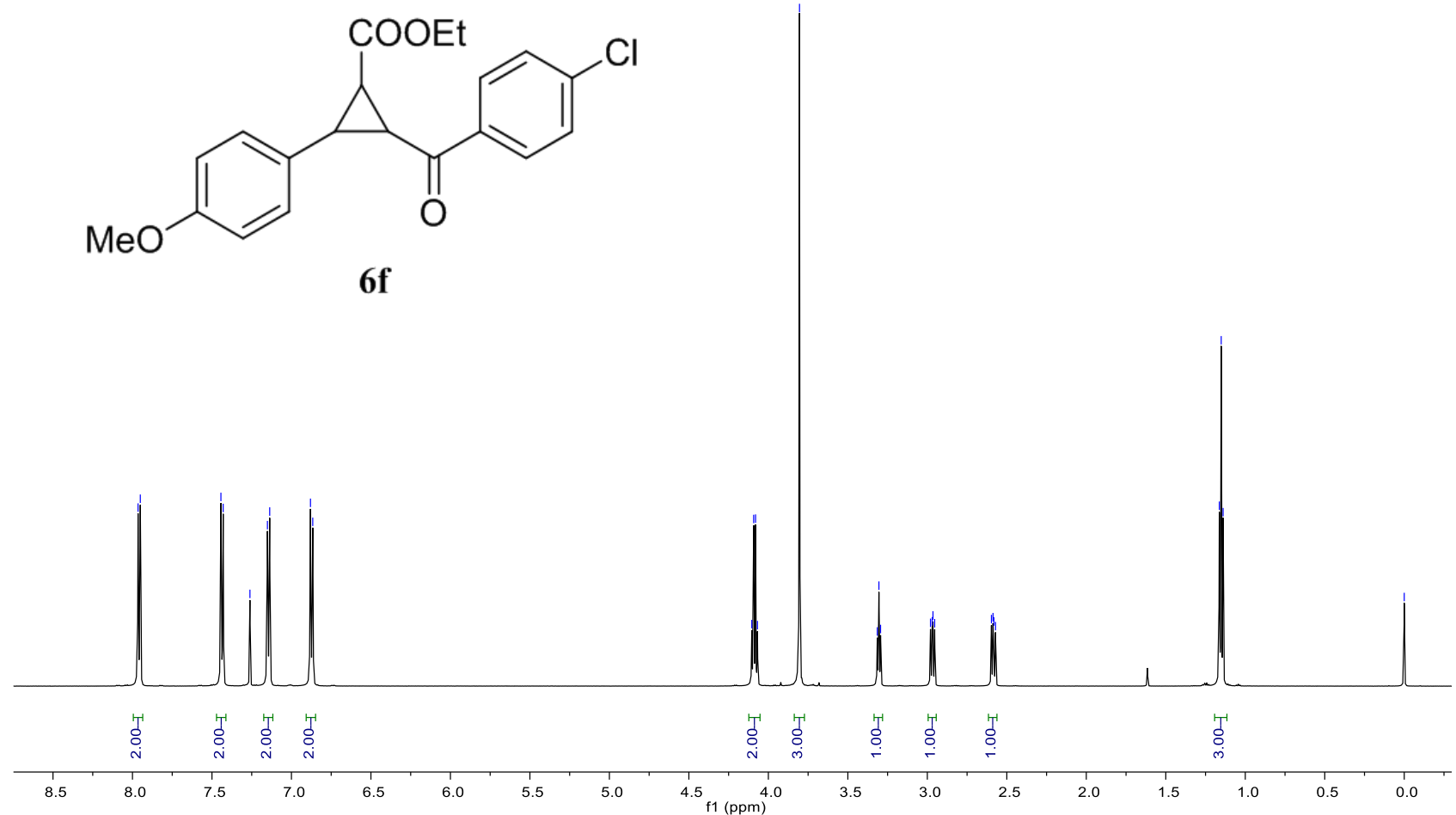

I

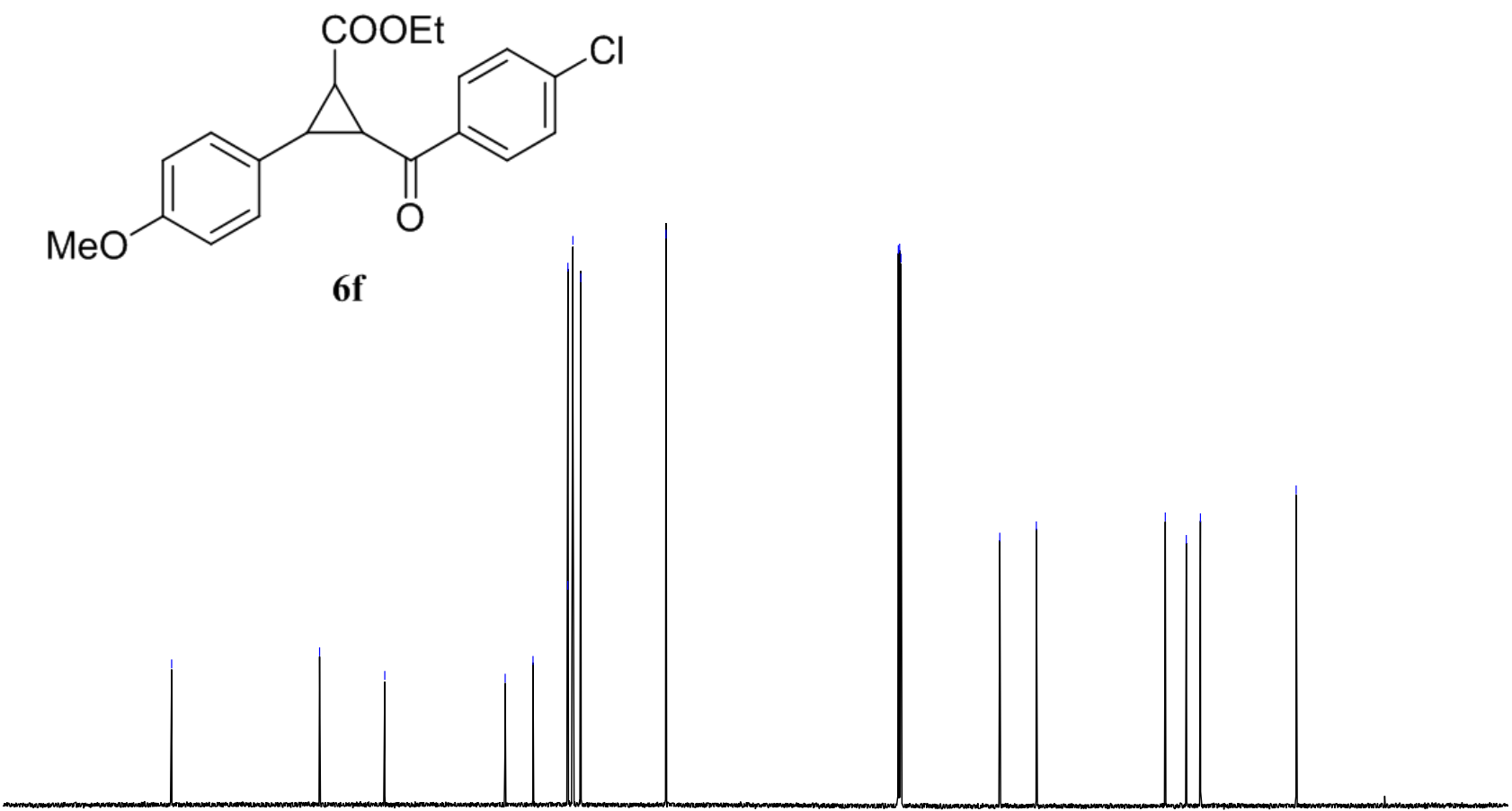

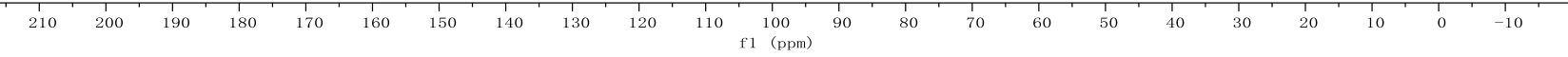




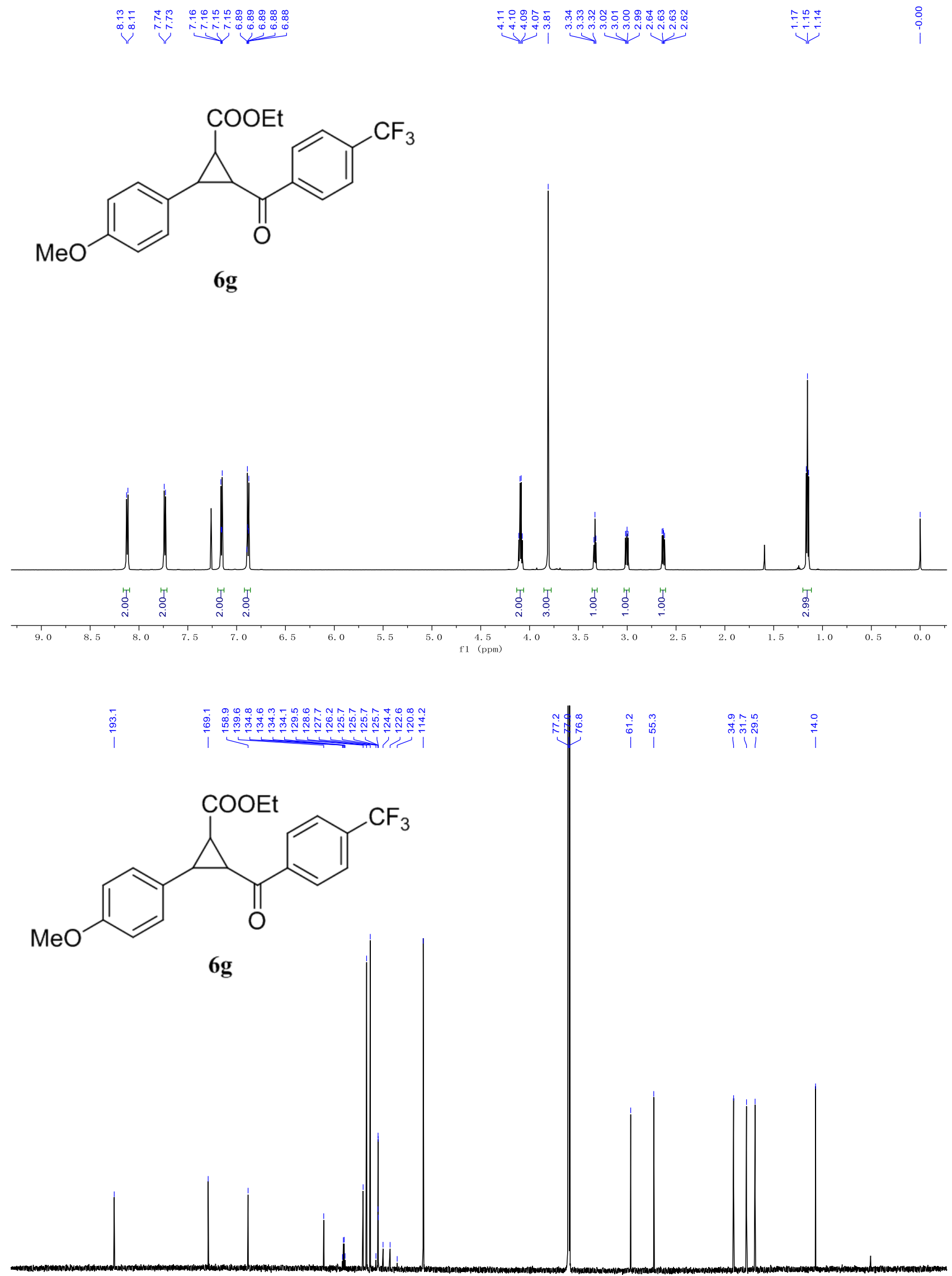

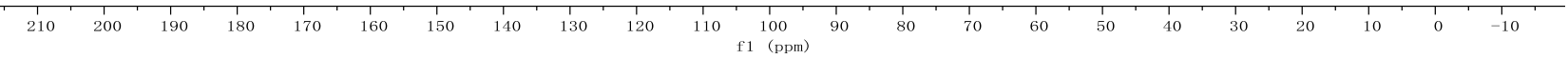


<smiles>CCOC(=O)C1C(C(=O)c2cccc(Cl)c2)C1c1ccc(OC)cc1</smiles>

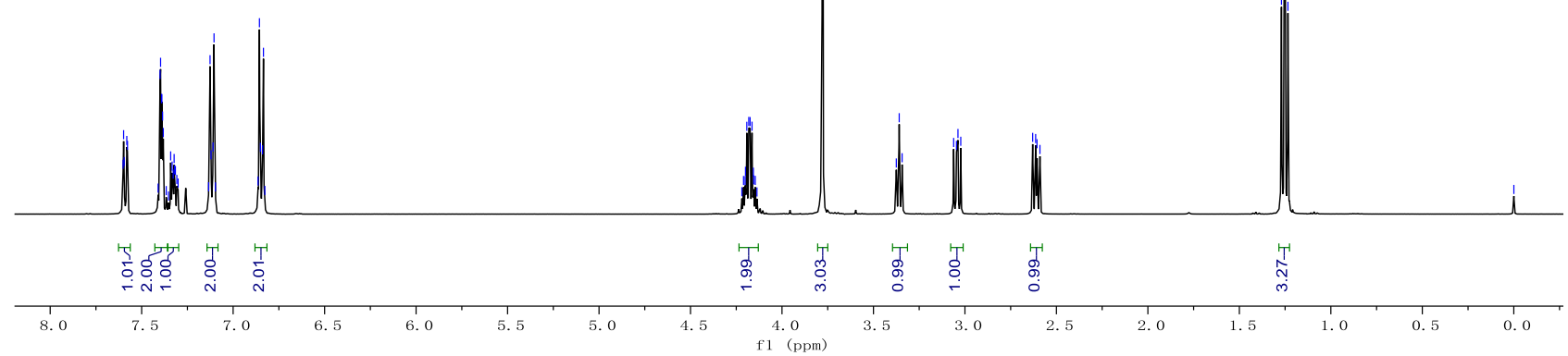

I
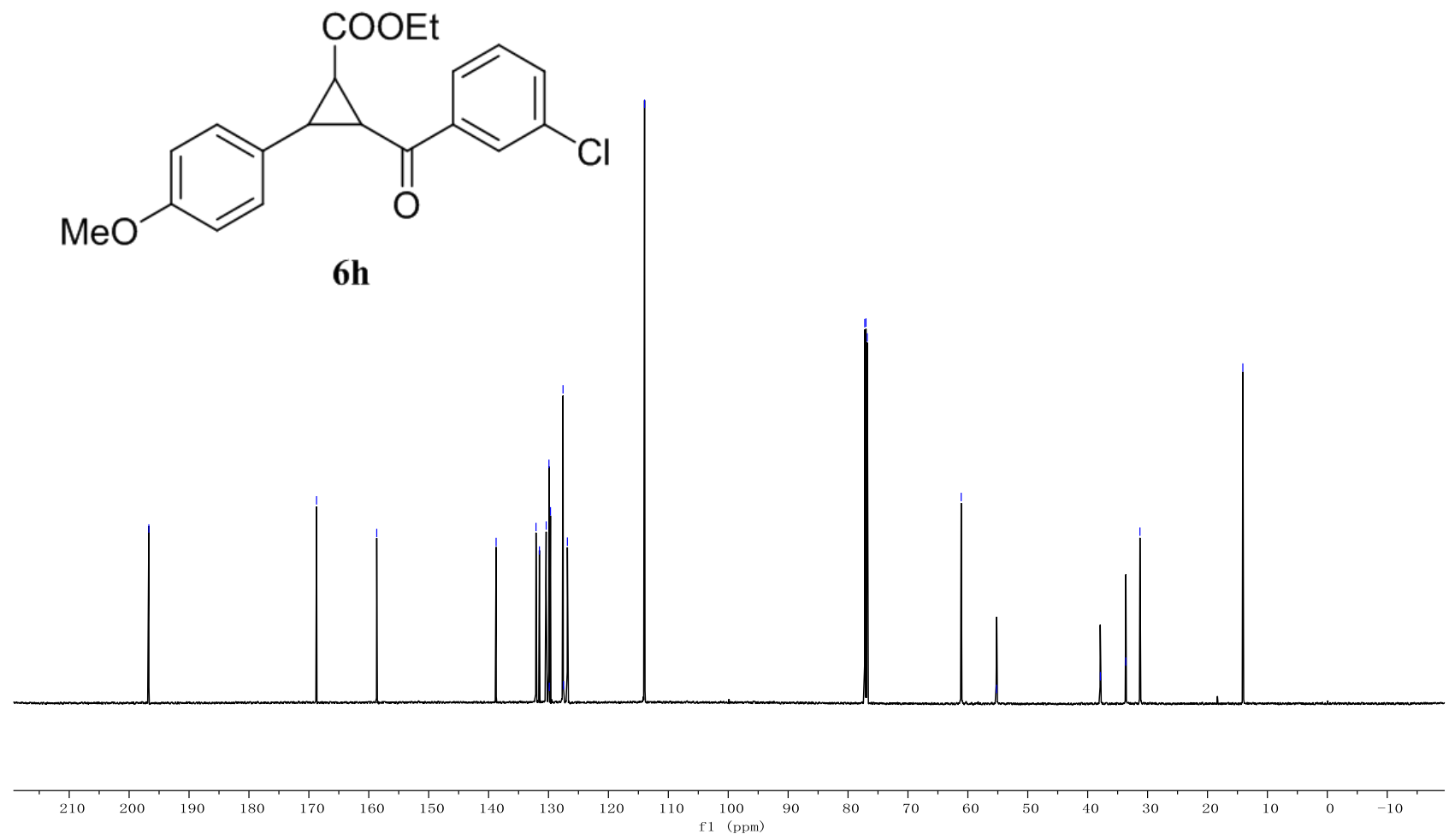
<smiles>CCOC(=O)C1C(C(C)=O)C1c1ccc(OC)cc1</smiles>

$6 i$

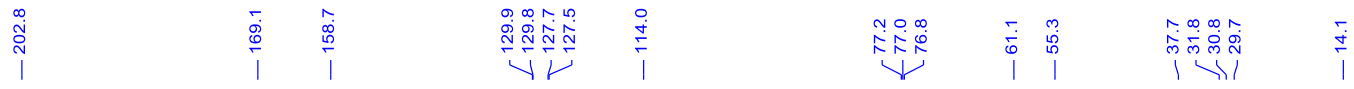

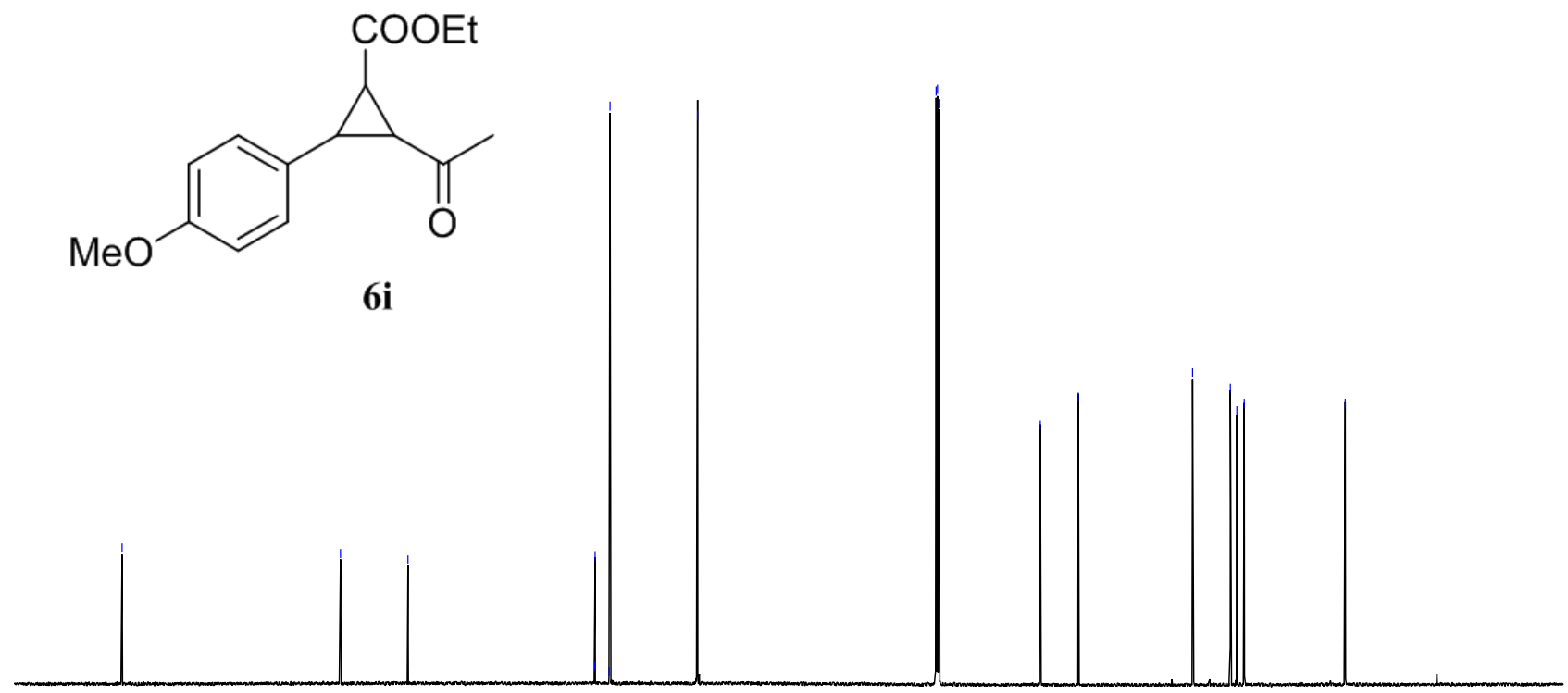

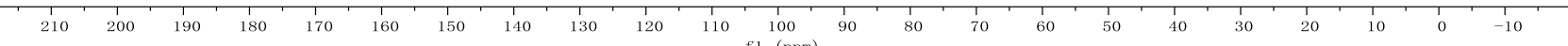




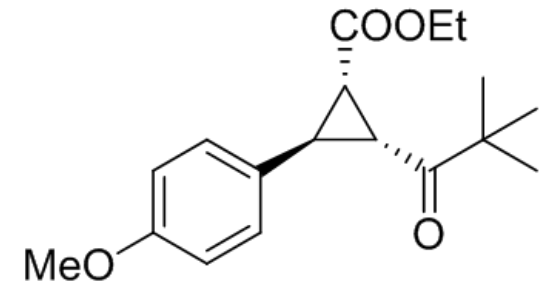

6j

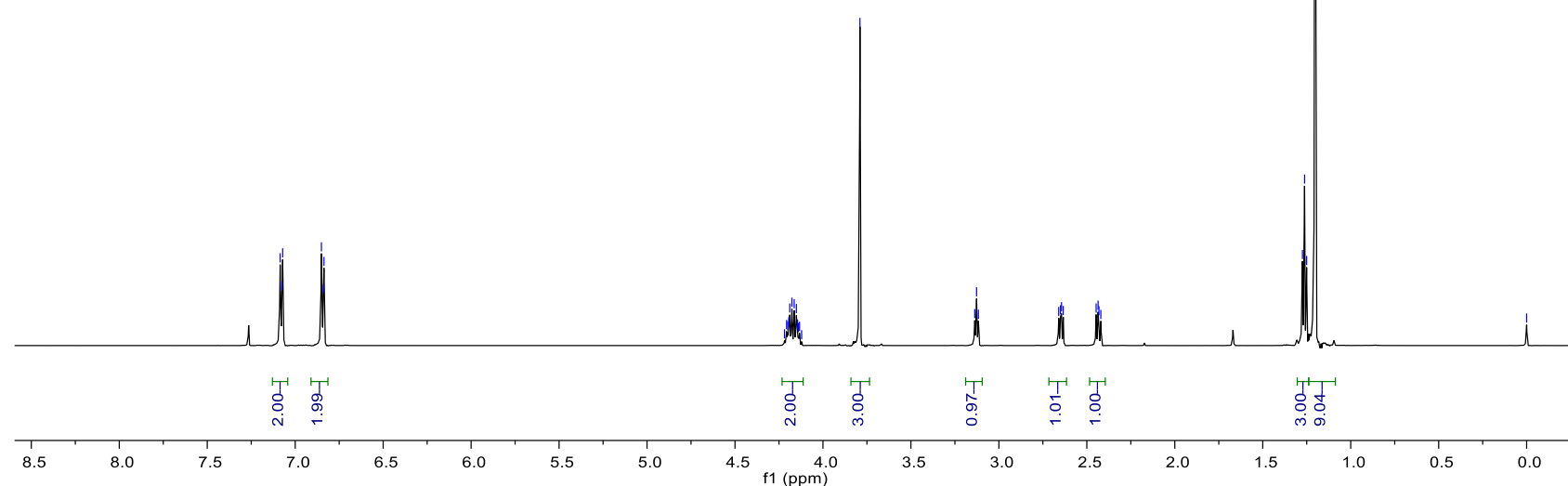

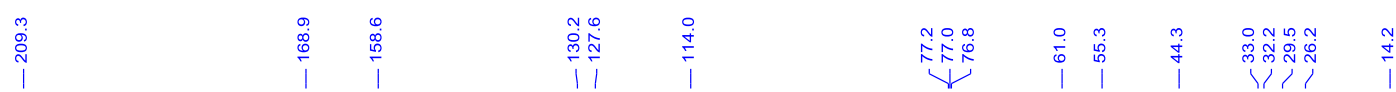

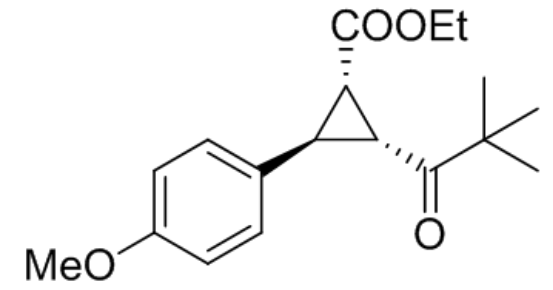

6j

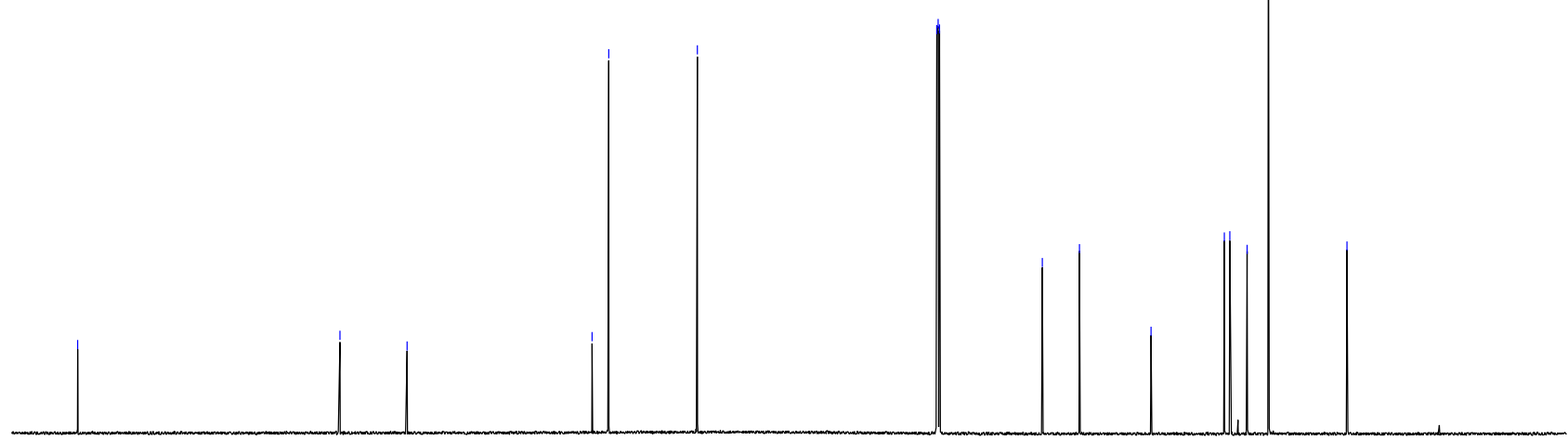

$\begin{array}{lllllllllllllllllllllllllllll}210 & 200 & 190 & 180 & 170 & 160 & 150 & 140 & 130 & 120 & 110 & 100 & 90 & 80 & 70 & 60 & 50 & 40 & 30 & 20 & 10 & 0 & -10\end{array}$ 

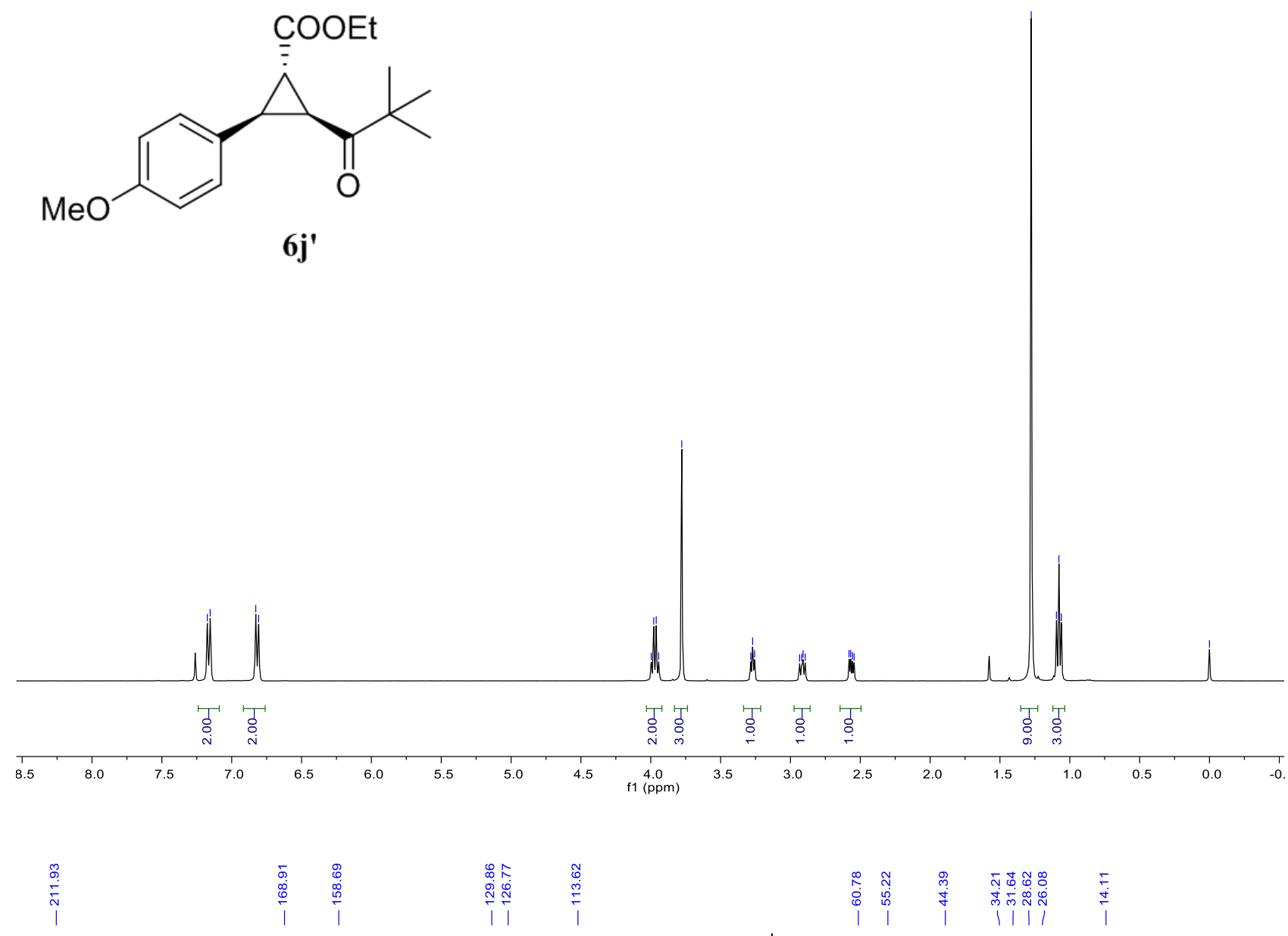

$\begin{array}{llllll}1 & 1 & \mid & 1 & 1 & 1\end{array}$
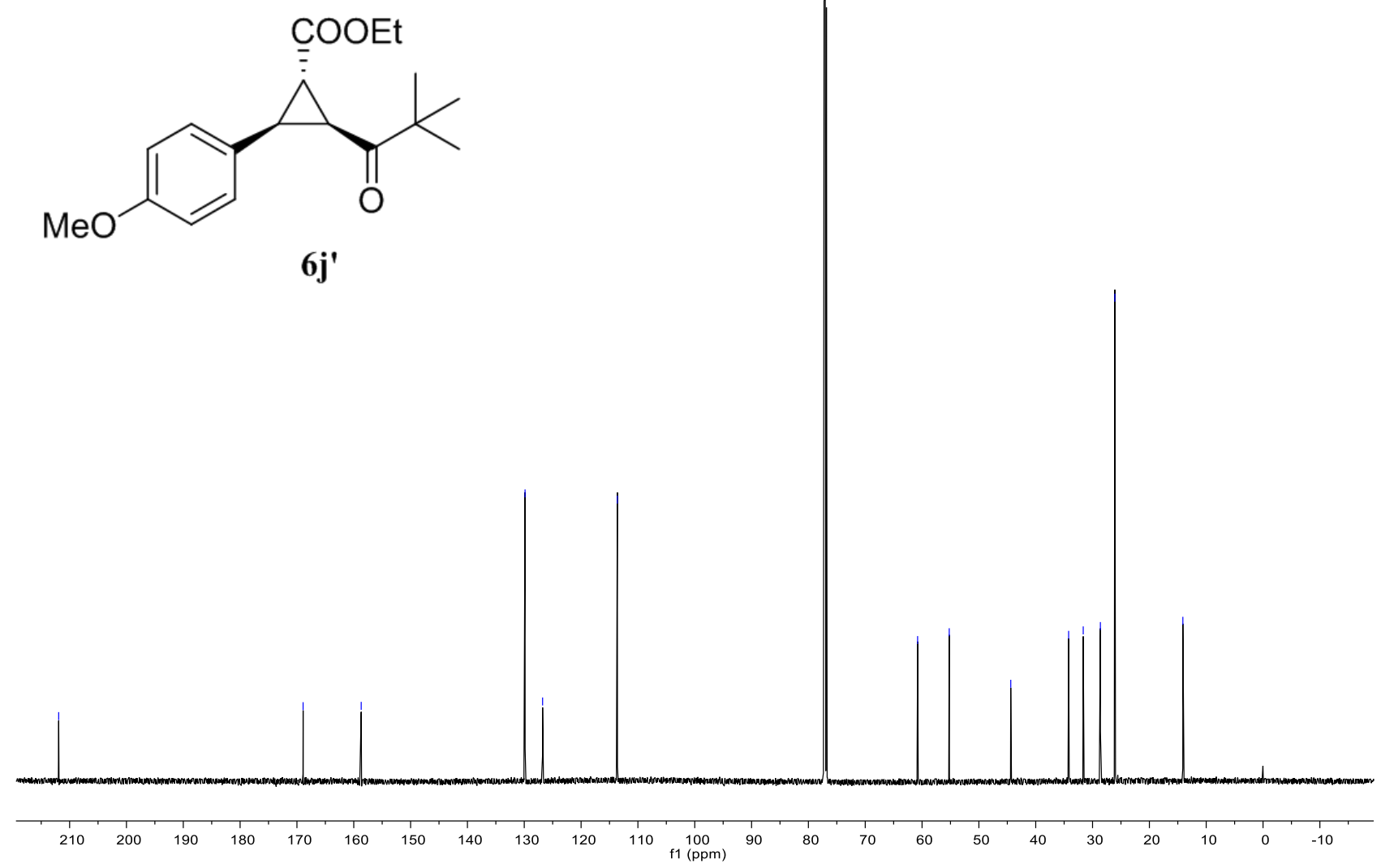
<smiles>CCOC(=O)C1C(c2ccc(OC)cc2)C12CCCCC2=O</smiles>

$6 k$

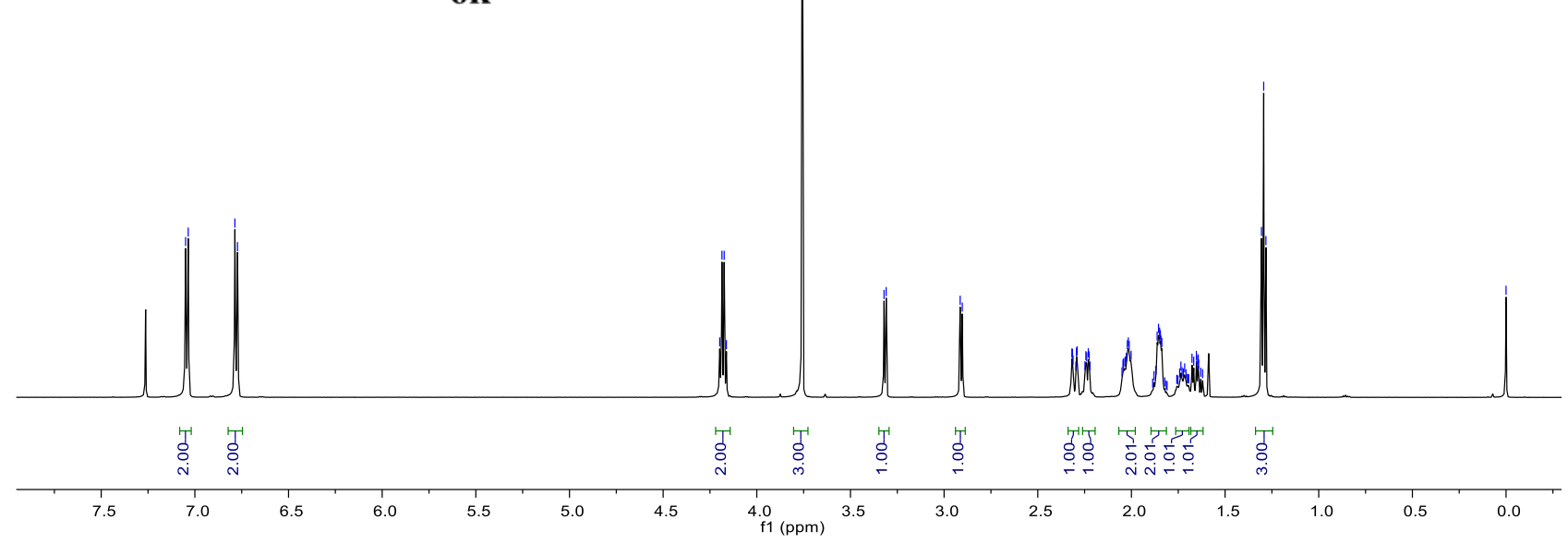

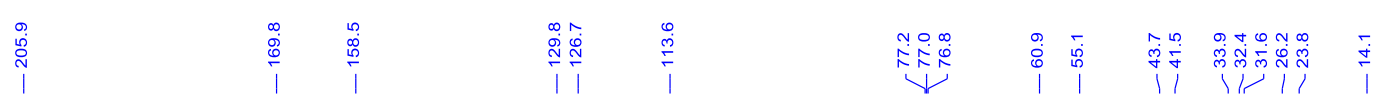

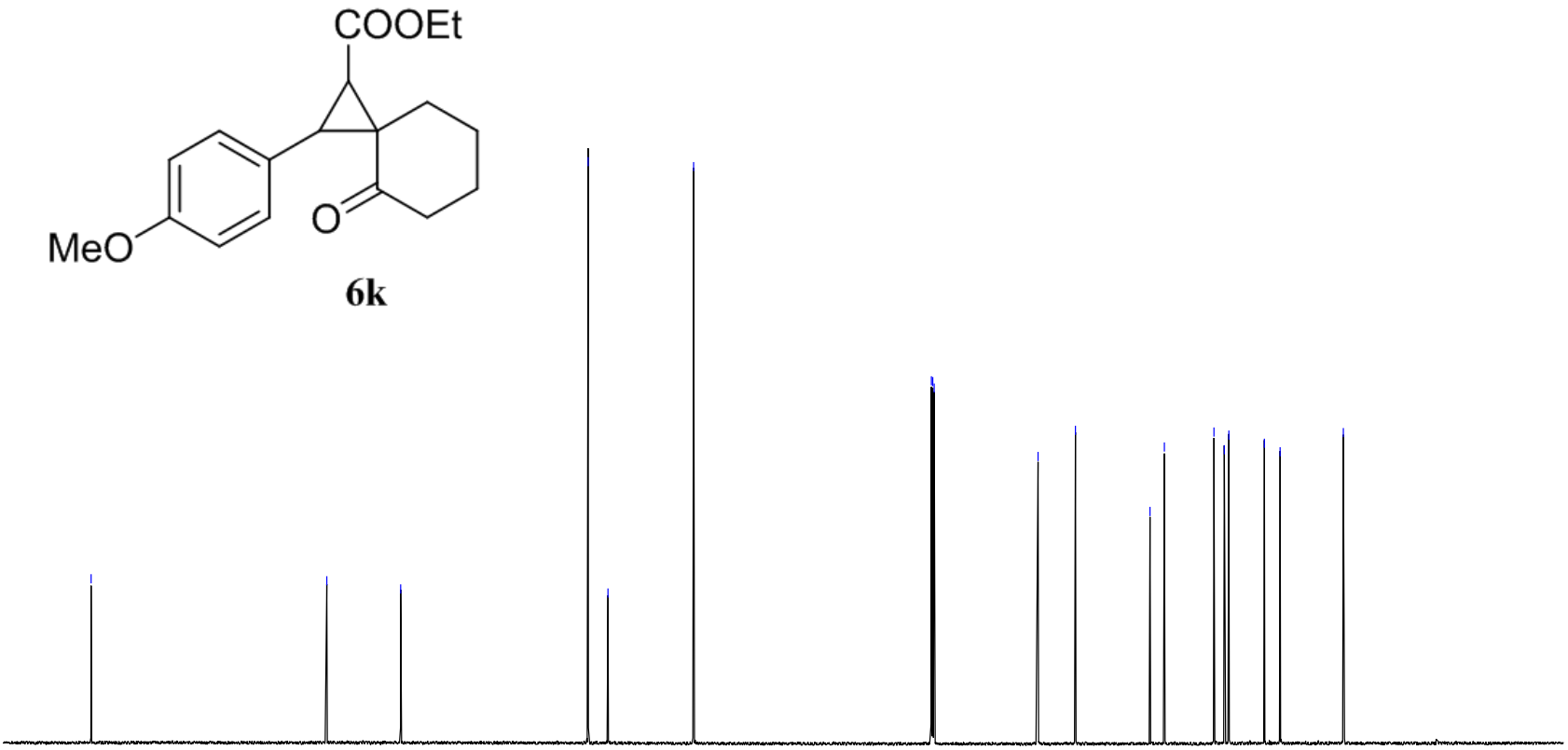

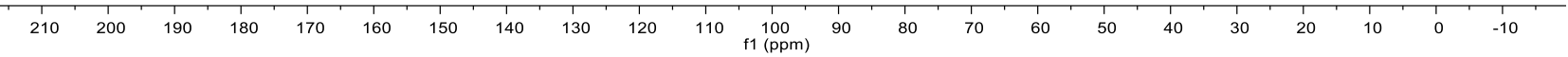


年

$\stackrel{\substack{\infty \\ \infty}}{\infty}$

$\stackrel{\circ}{\circ}$

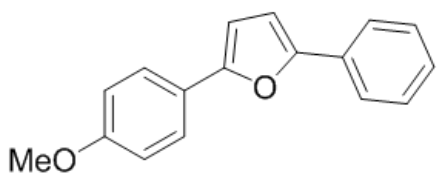

$4 a$

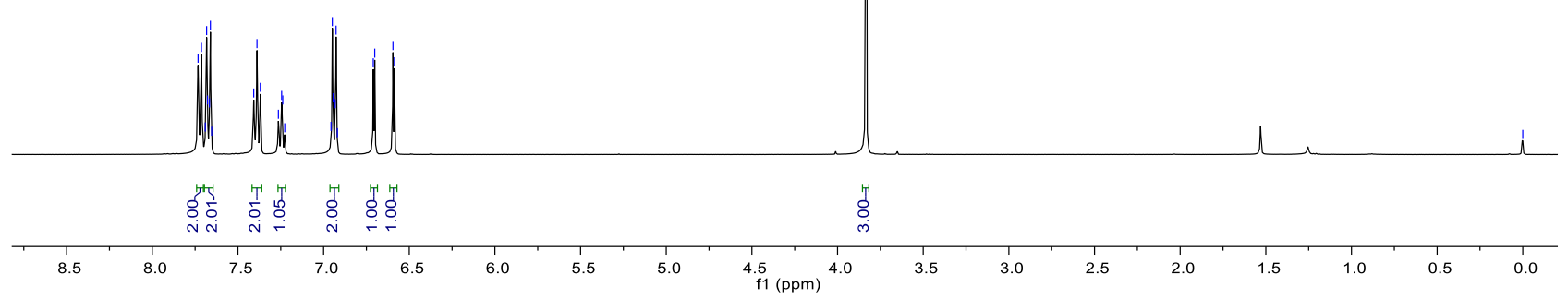

i

لَivi

$\sqrt{\sqrt[n]{n+50}}$

$\underset{\substack{\infty \\ \infty}}{\infty}$

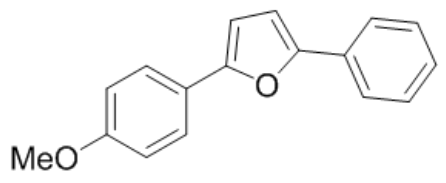

$4 a$
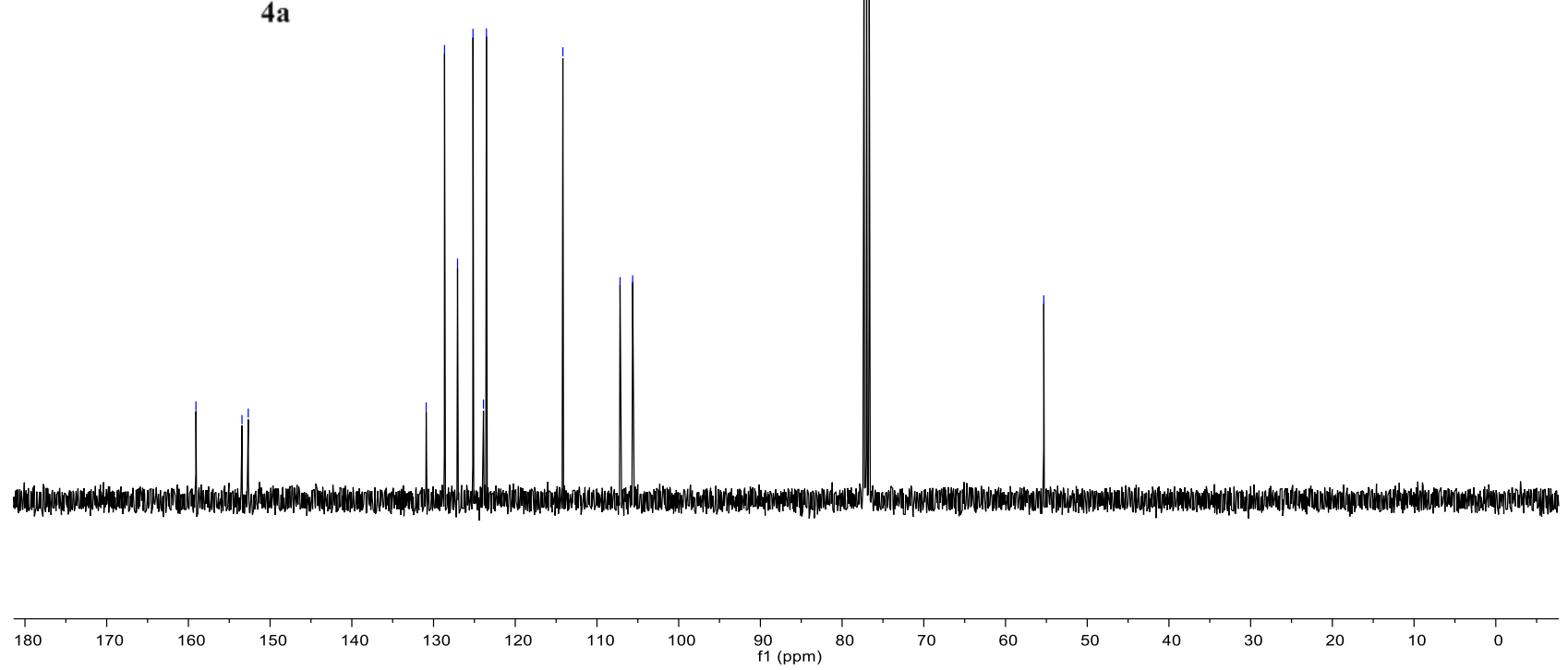

S41 


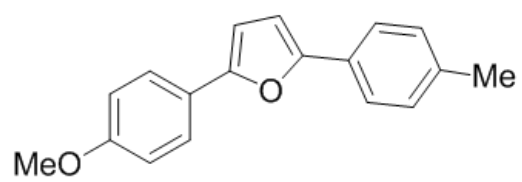

4b

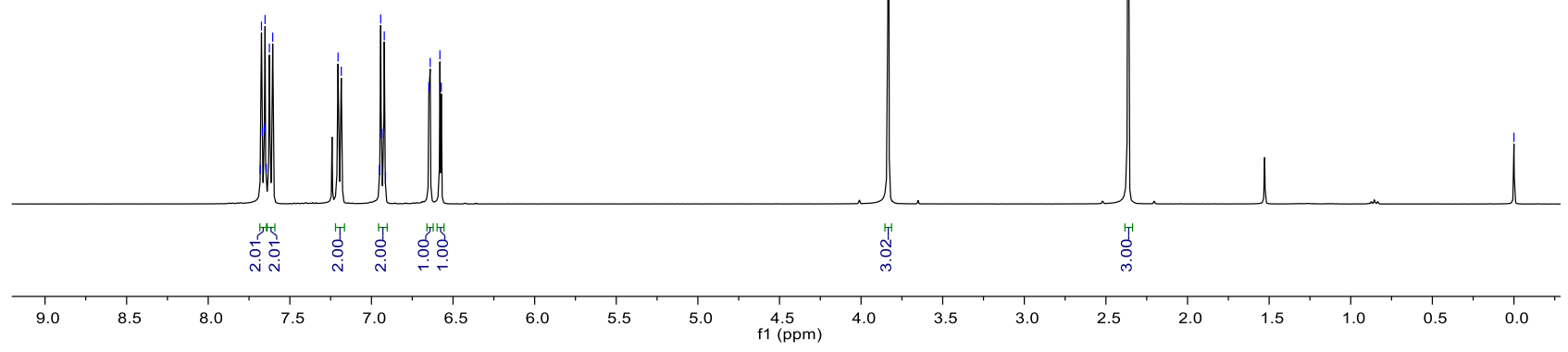

|

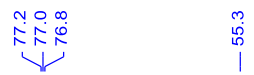

$\stackrel{m}{i}$

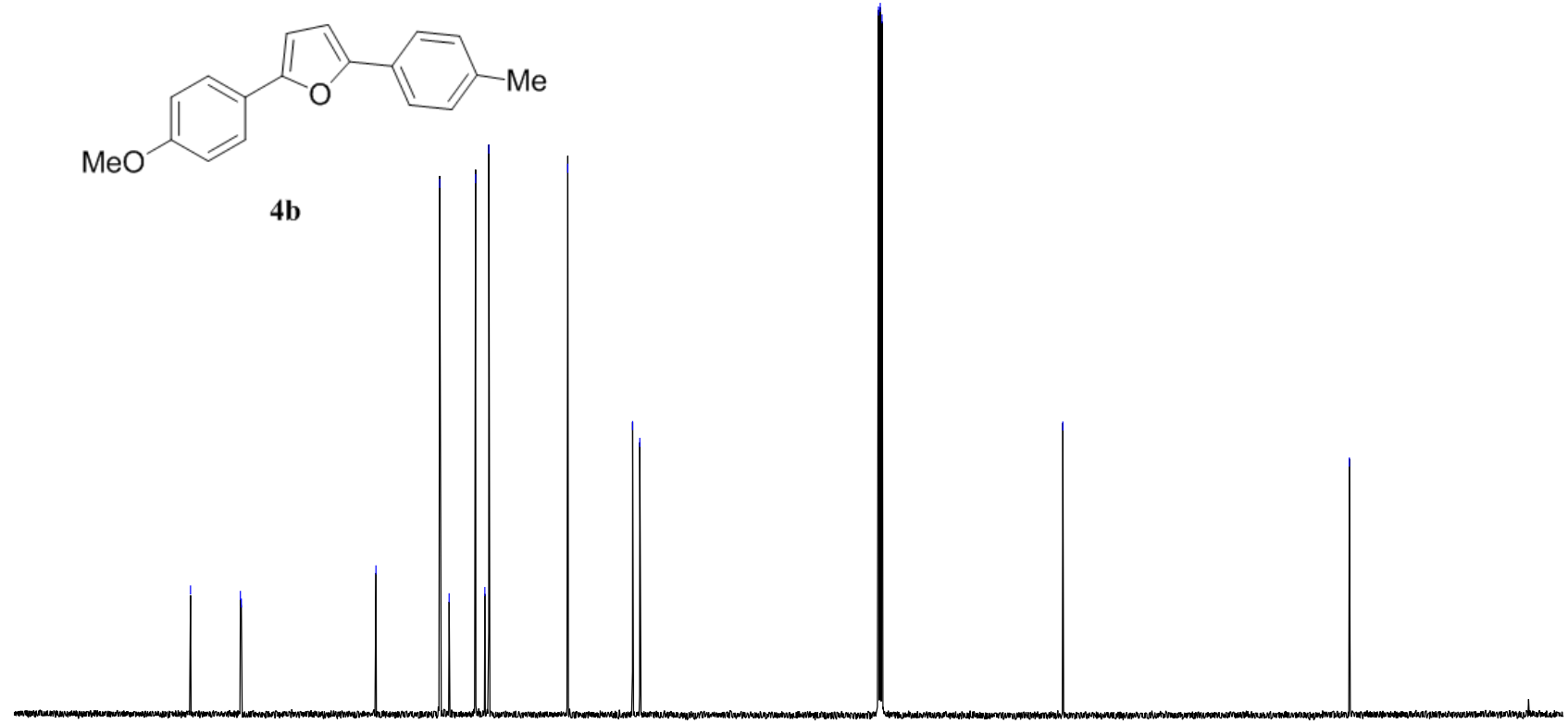

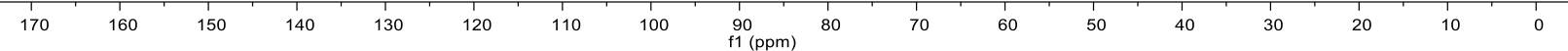




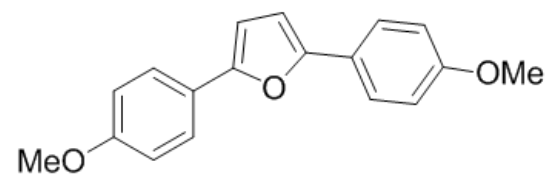

$4 c$
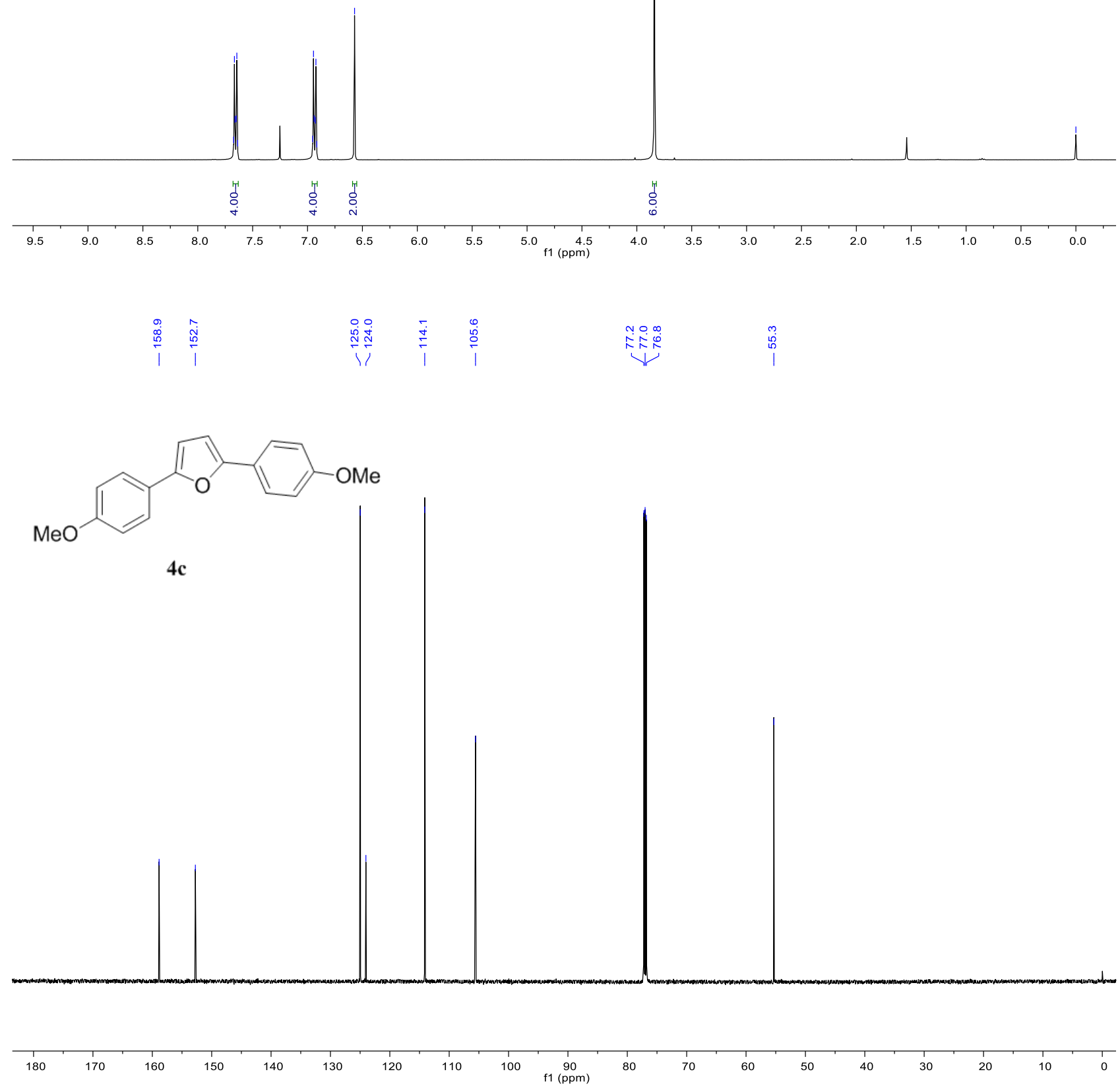
<smiles>COc1ccc(-c2ccc(-c3ccc(C(C)(C)C)cc3)o2)cc1</smiles>
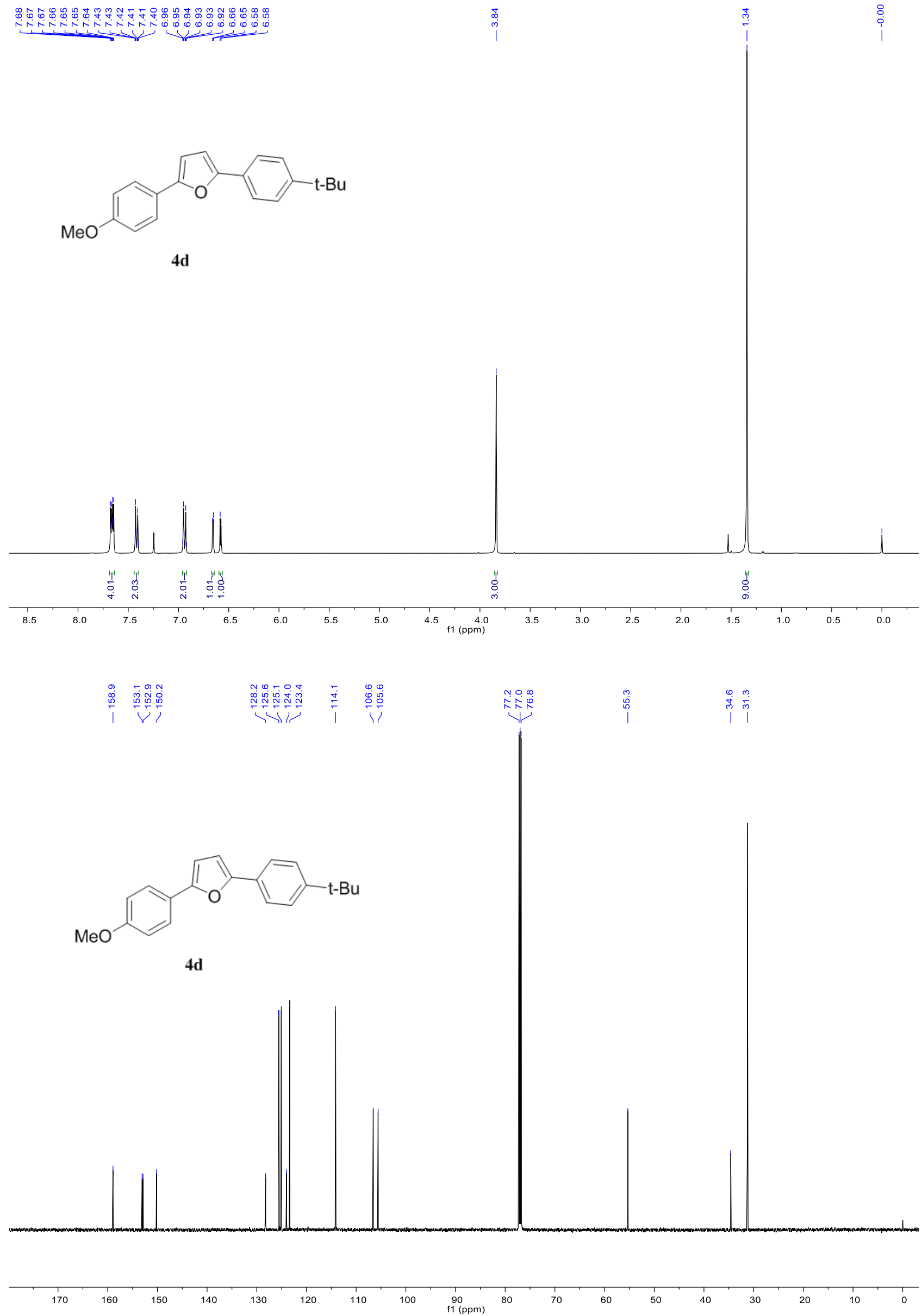


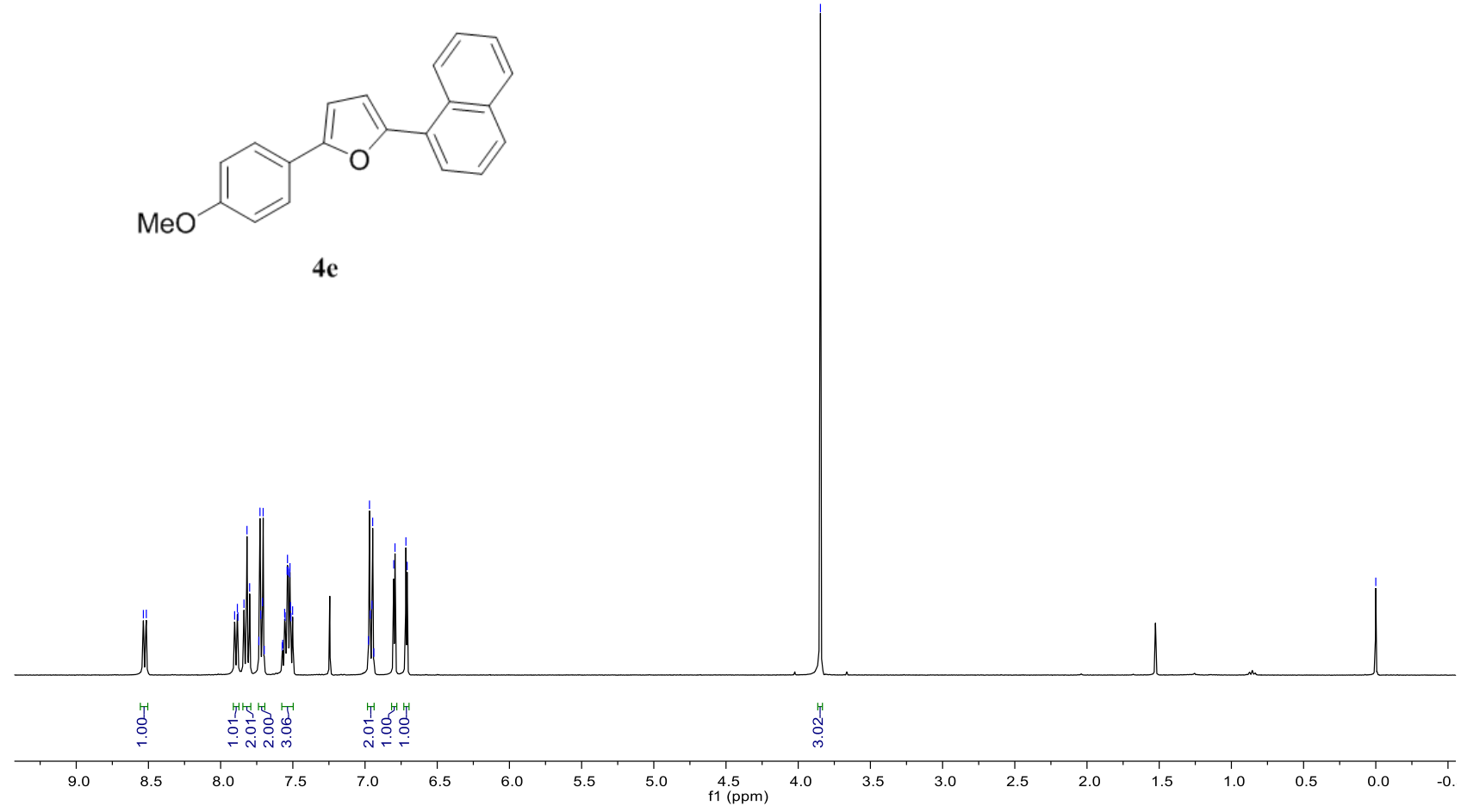

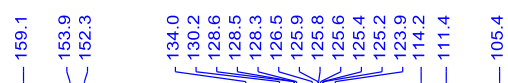

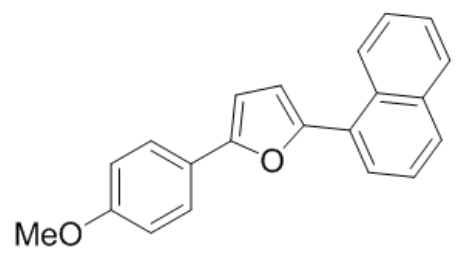

$4 e$
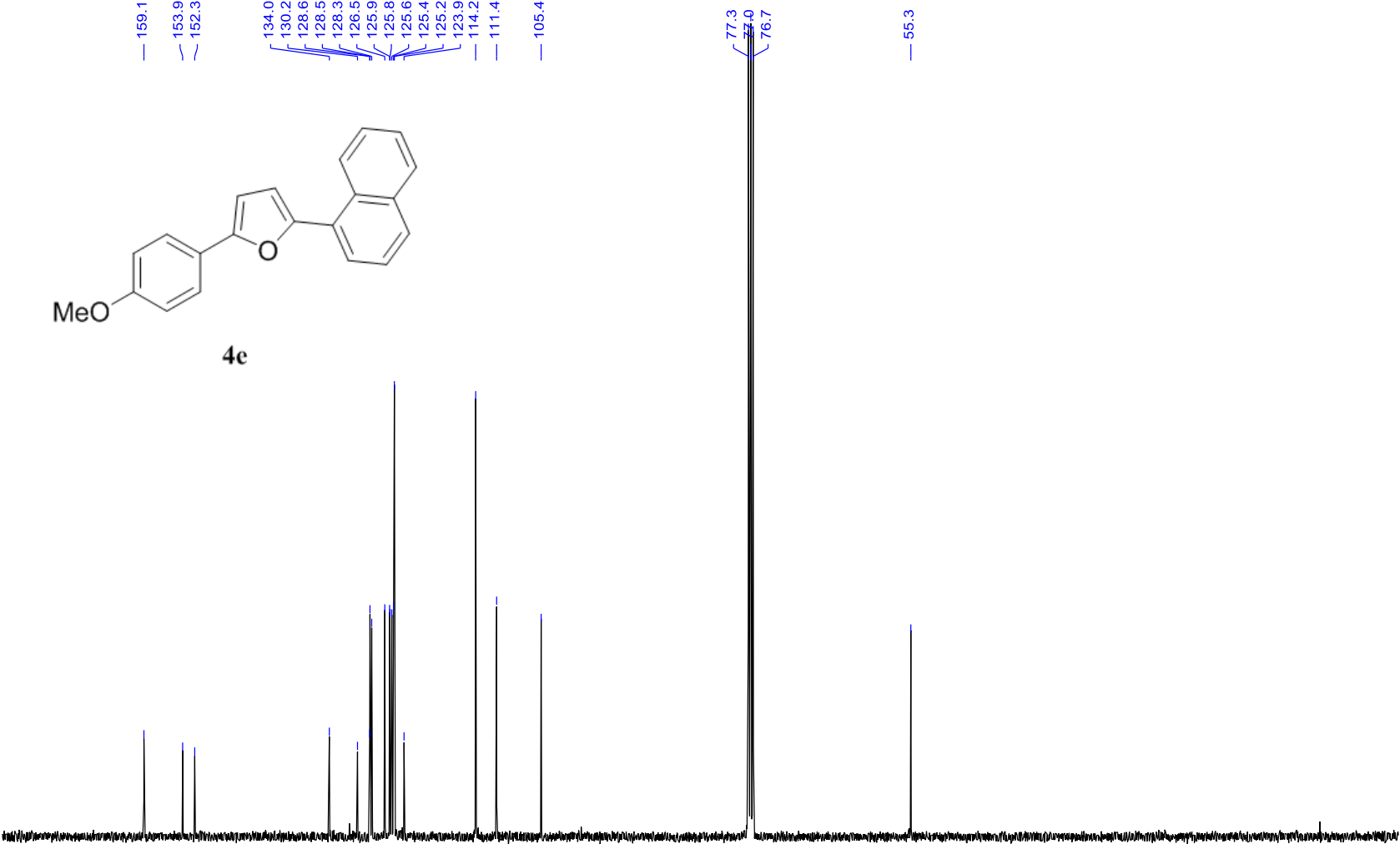

$\begin{array}{lllllllll}170 & 160 & 150 & 140 & 130 & 120 & 110 & 100 & 90\end{array}$ 


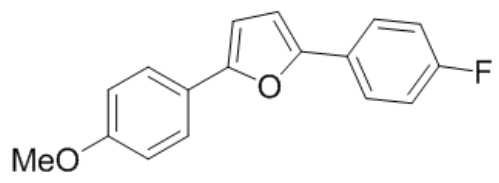

4f

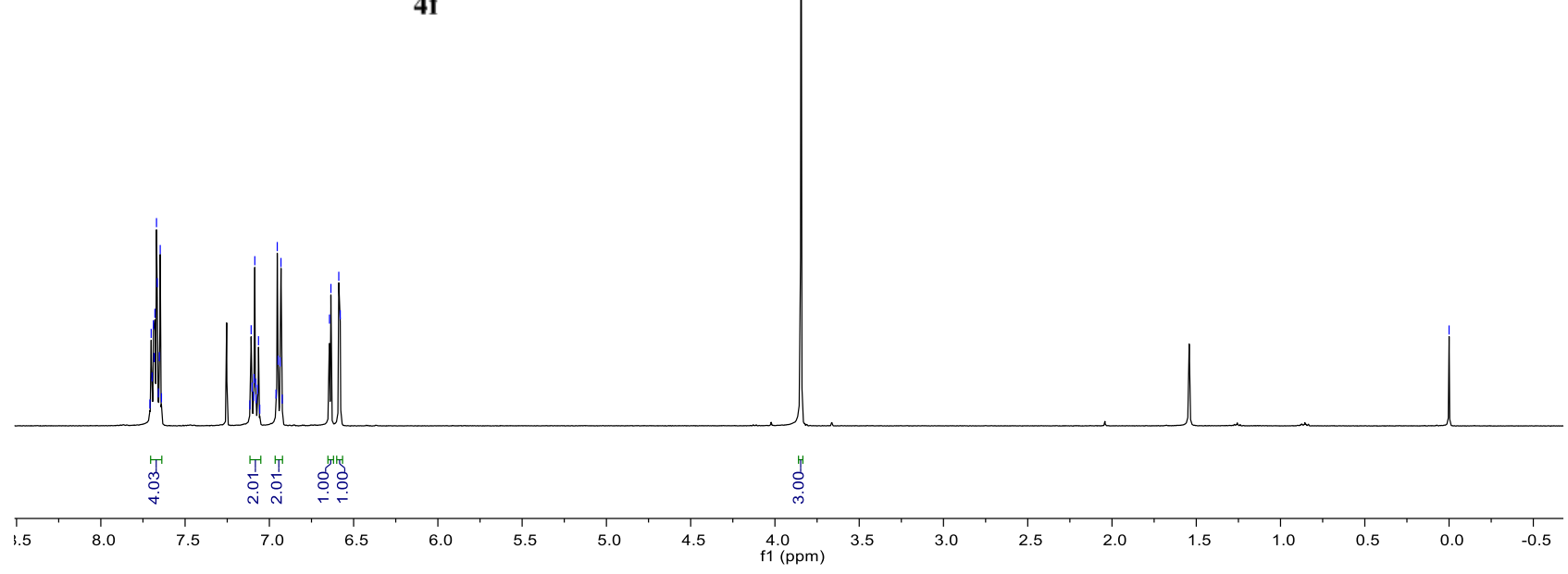

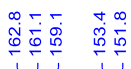

$\underbrace{-1}$

1111

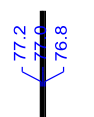

蕰品<smiles>COc1ccc(-c2ccc(-c3ccc(F)cc3)o2)cc1</smiles>

$4 f$
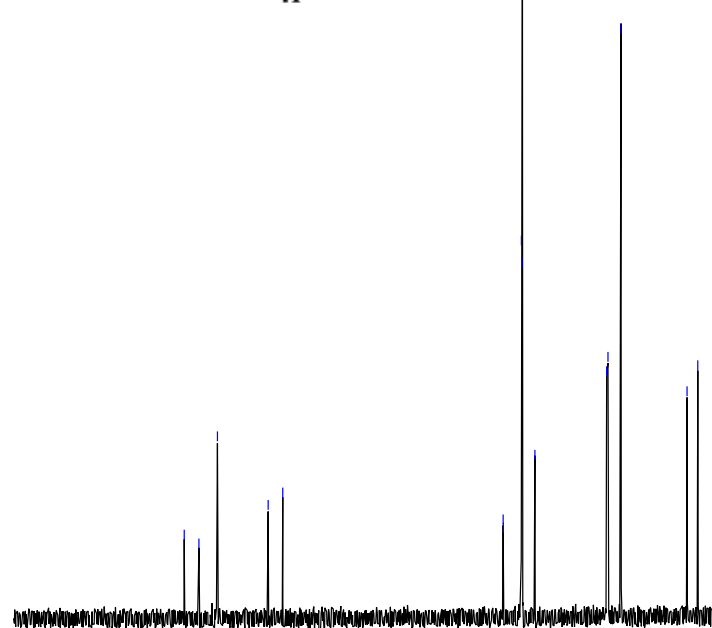

180

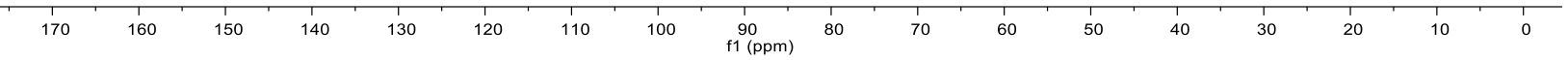


<smiles>COc1ccc(-c2ccc(-c3ccc(Cl)cc3)o2)cc1</smiles>

$4 \mathrm{~g}$

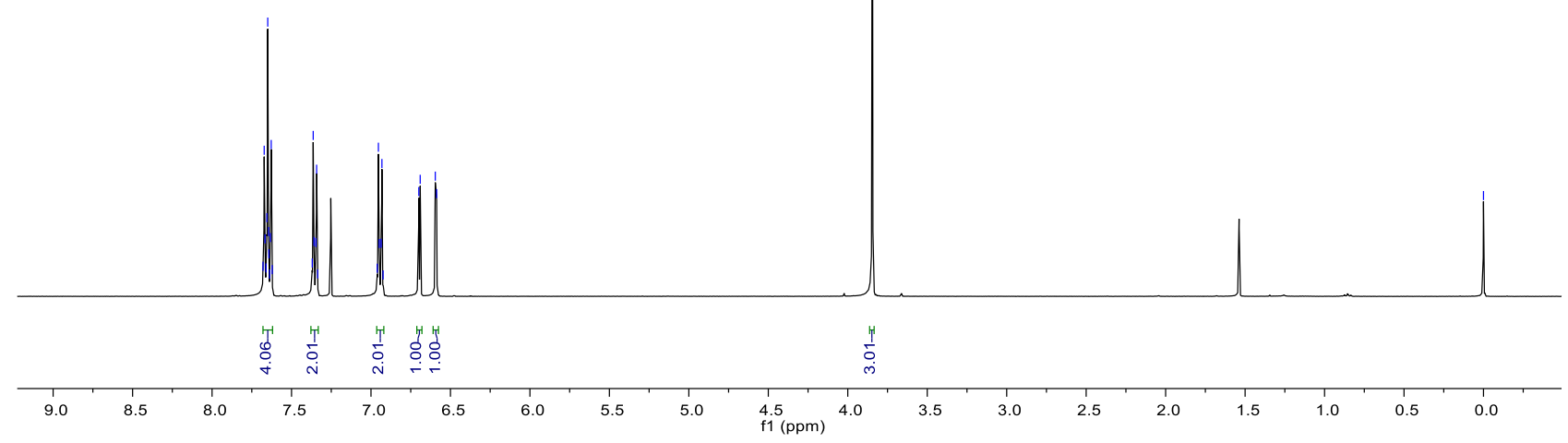

1)

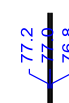

总<smiles>COc1ccc(-c2ccc(-c3ccc(Cl)cc3)o2)cc1</smiles>

$4 \mathrm{~g}$

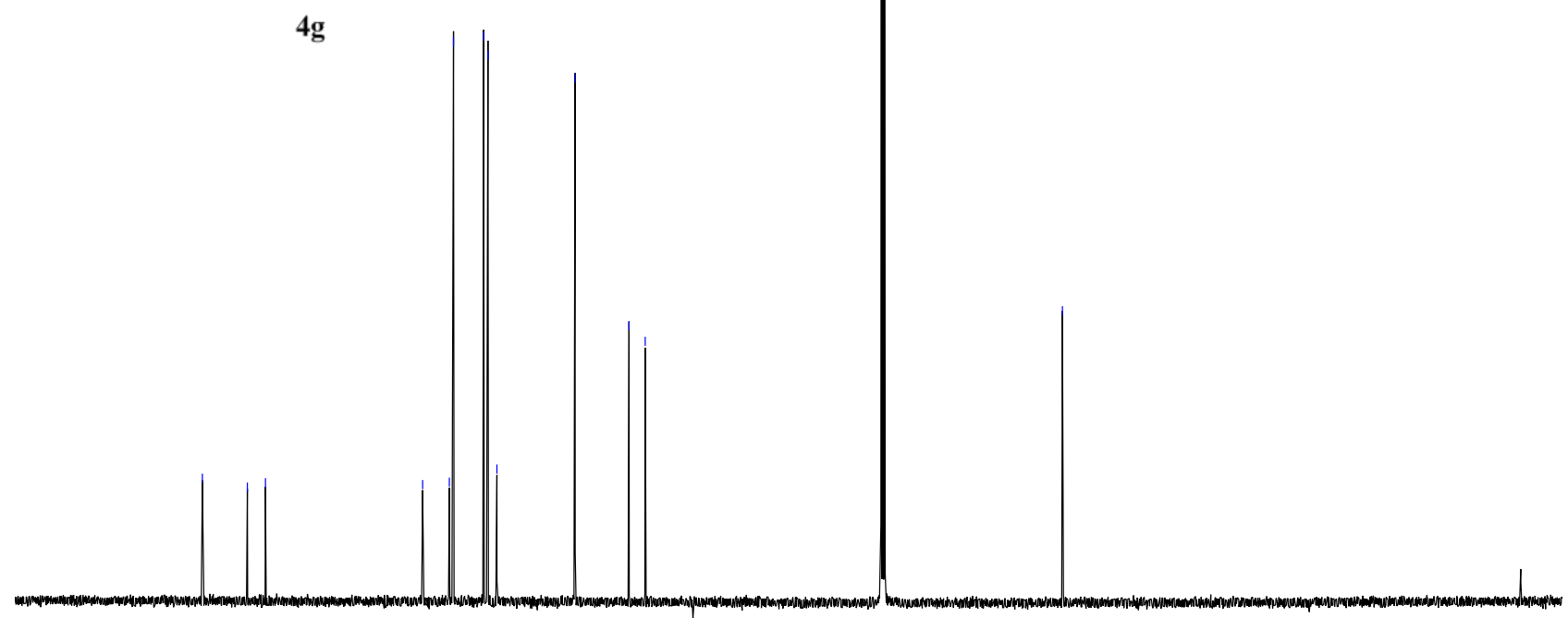




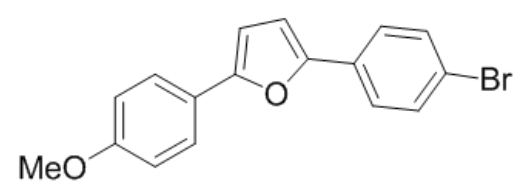

4h

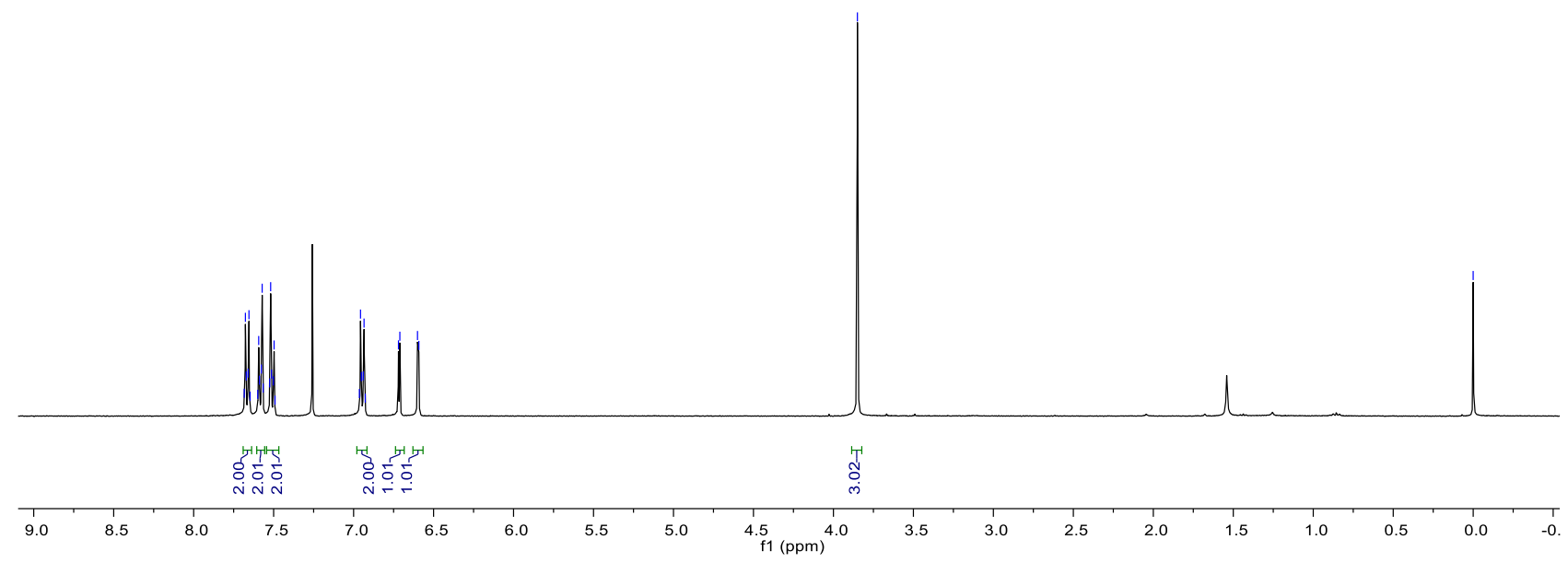

li

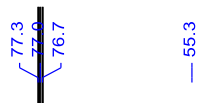<smiles>COc1ccc(-c2ccc(-c3ccc(Br)cc3)o2)cc1</smiles>

4h

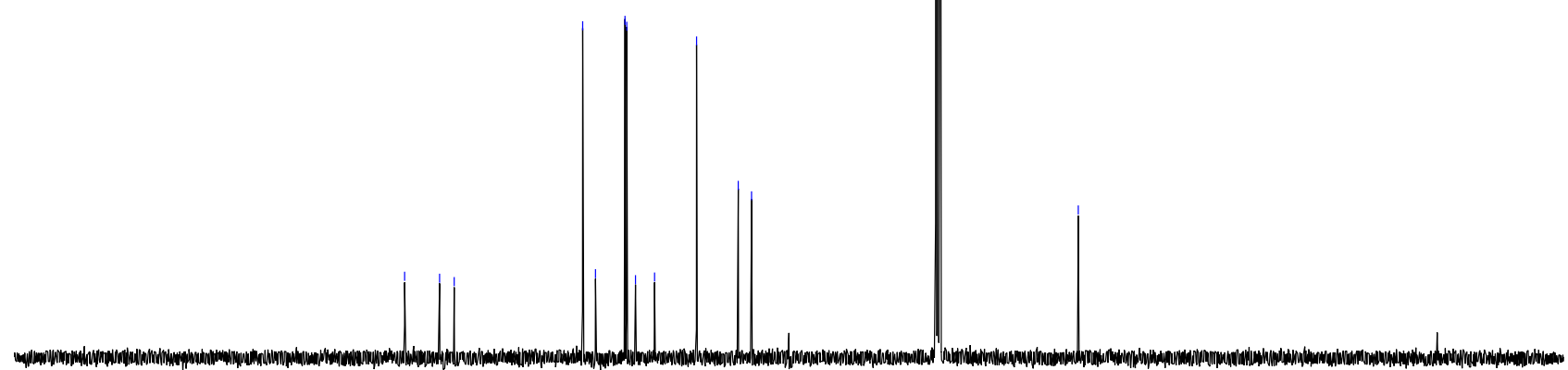

$\begin{array}{llllllllllllllllllllllllllll}210 & 200 & 190 & 180 & 170 & 160 & 150 & 140 & 130 & 120 & 110 & 100 & 90 & 80 & 70 & 60 & 50 & 40 & 30 & 20 & 10 & 0 & -10 & \end{array}$ 
<smiles>COc1ccc(-c2ccc(-c3ccc(C(F)(F)F)cc3)o2)cc1</smiles>

$4 i$
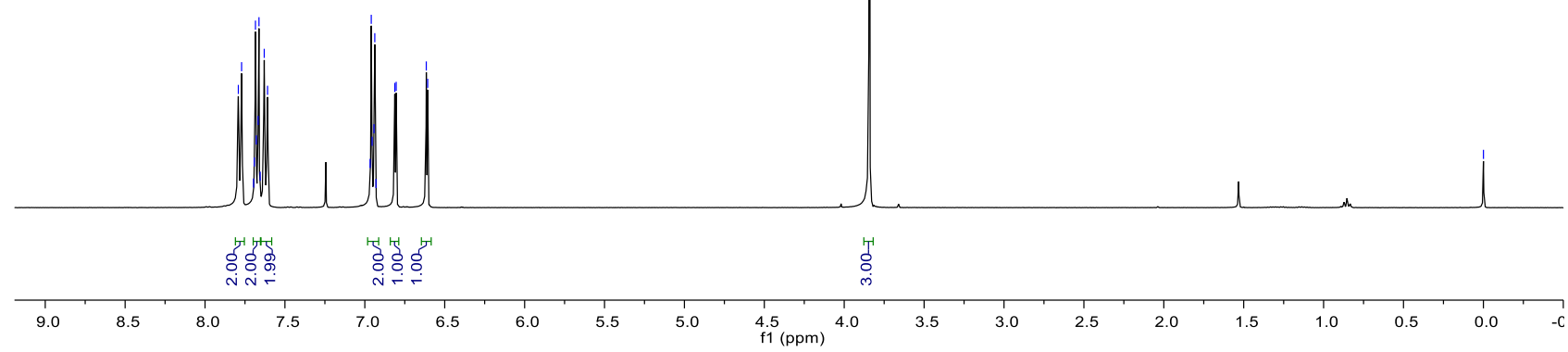

|<smiles>COc1ccc(-c2ccc(-c3ccc(C(F)(F)F)cc3)o2)cc1</smiles>

$4 \mathbf{i}$

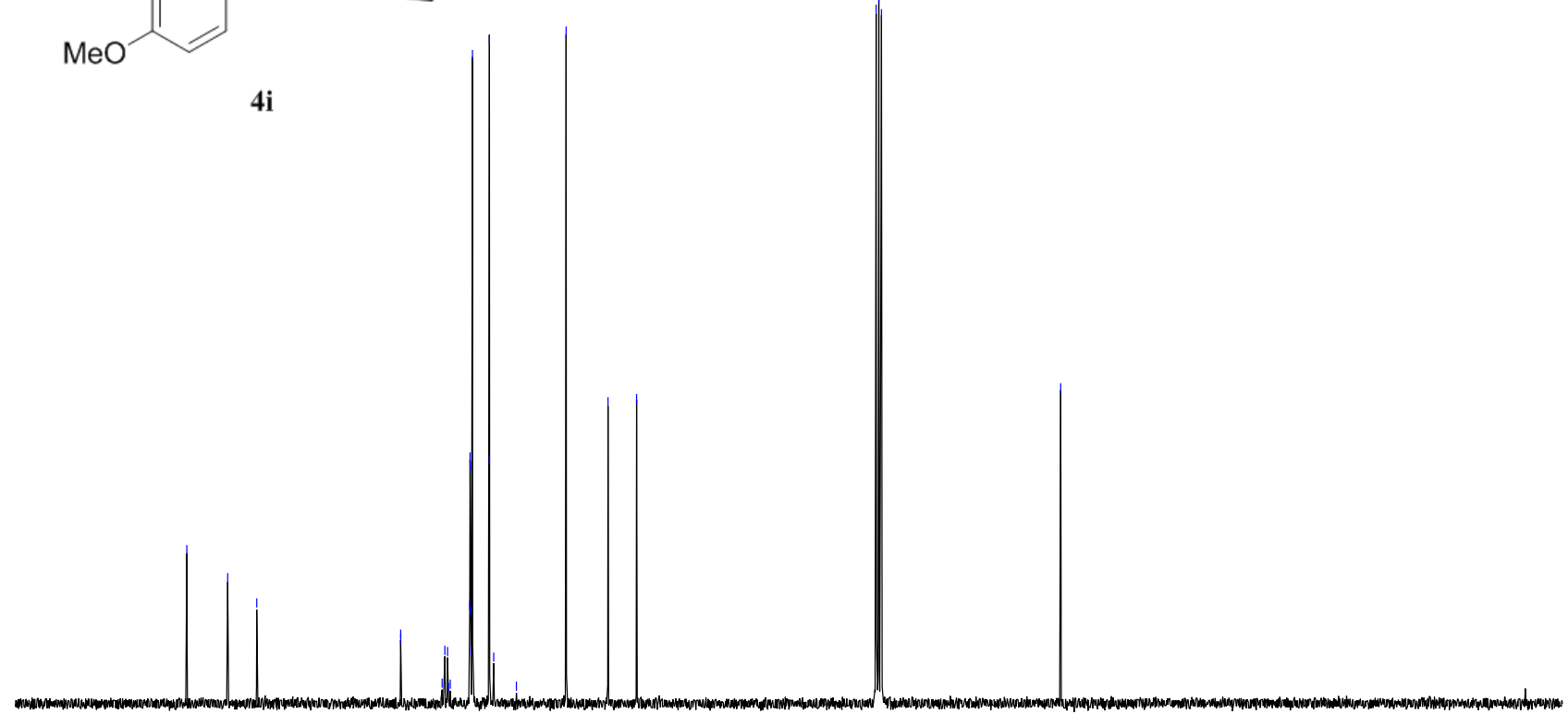

170

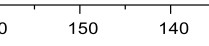

$130 \quad 120$

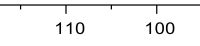

$\underset{f 1(\mathrm{ppm})}{90} 80$

60

50

$\begin{array}{lllll}1 & 1 & 1 & 1 & 1 \\ 40 & 20 & 10 & 0\end{array}$ 


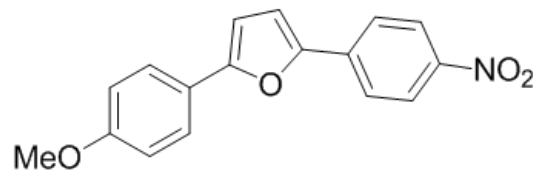

4j

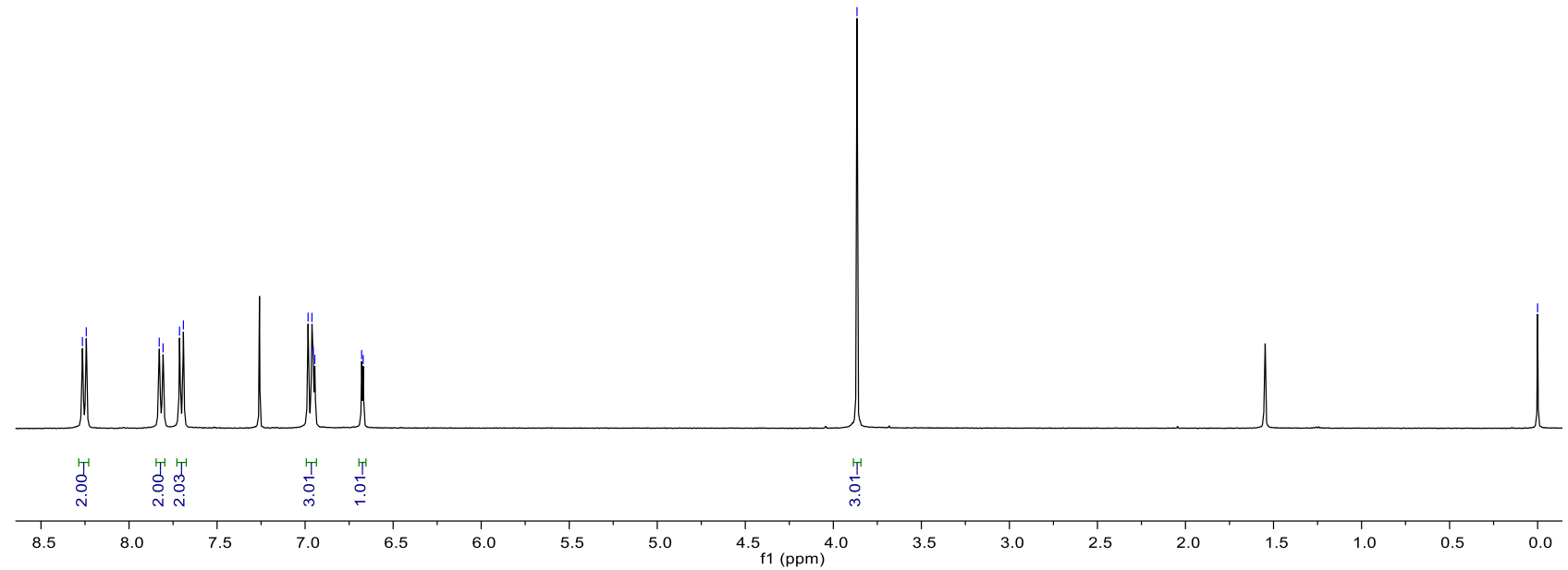

j<smiles>COc1ccc(-c2ccc(-c3ccc([N+](=O)[O-])cc3)o2)cc1</smiles>

$4 j$

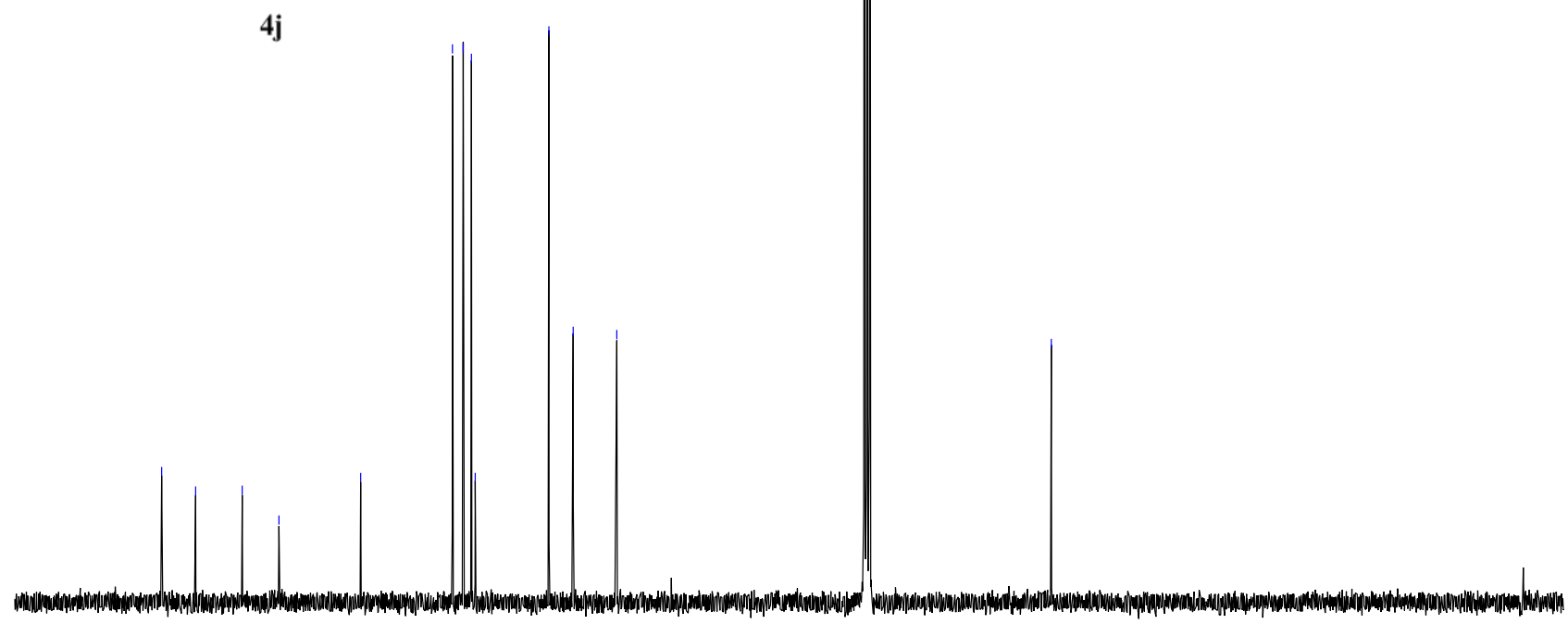

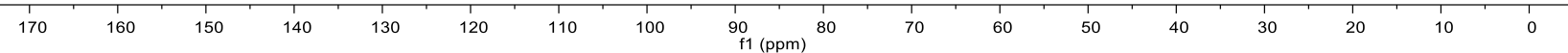




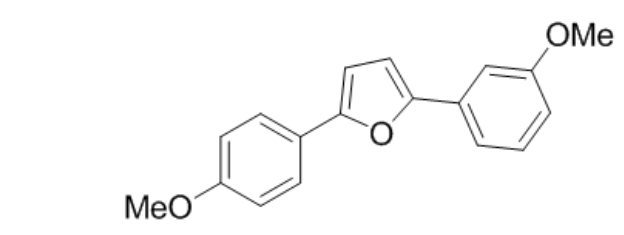

$4 k$

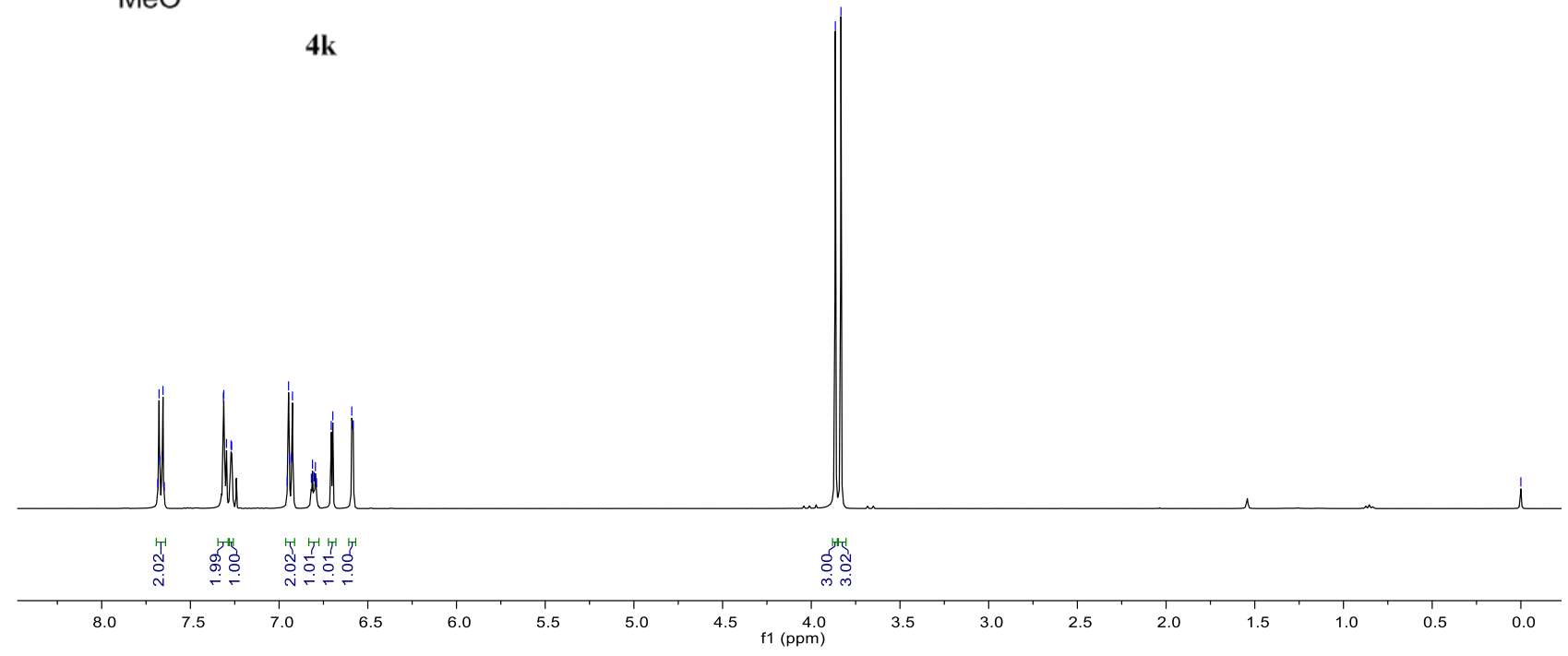

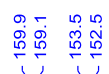

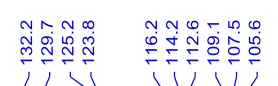

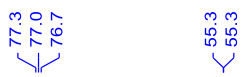

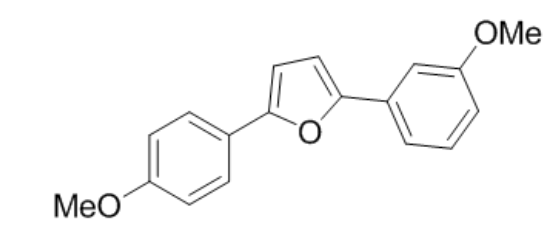

$4 k$

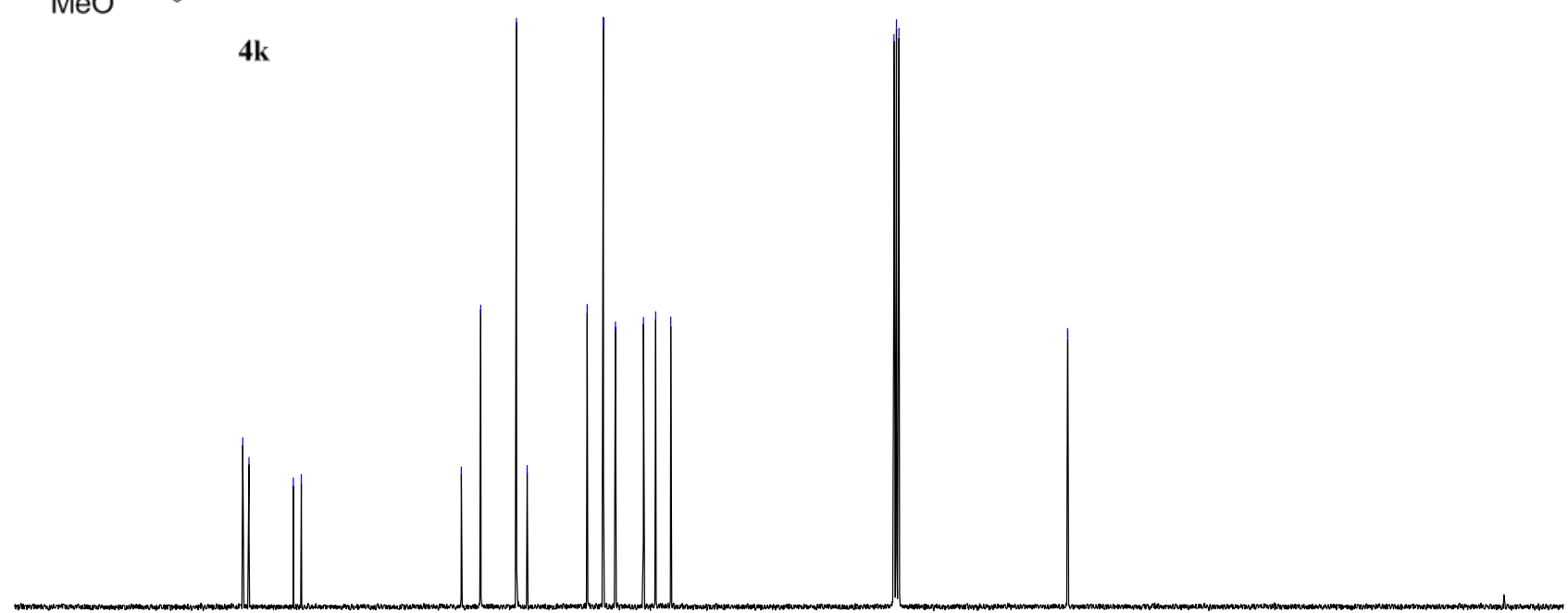

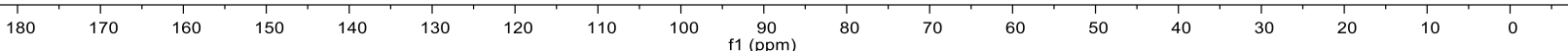




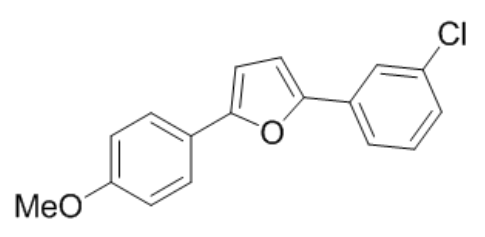

4I
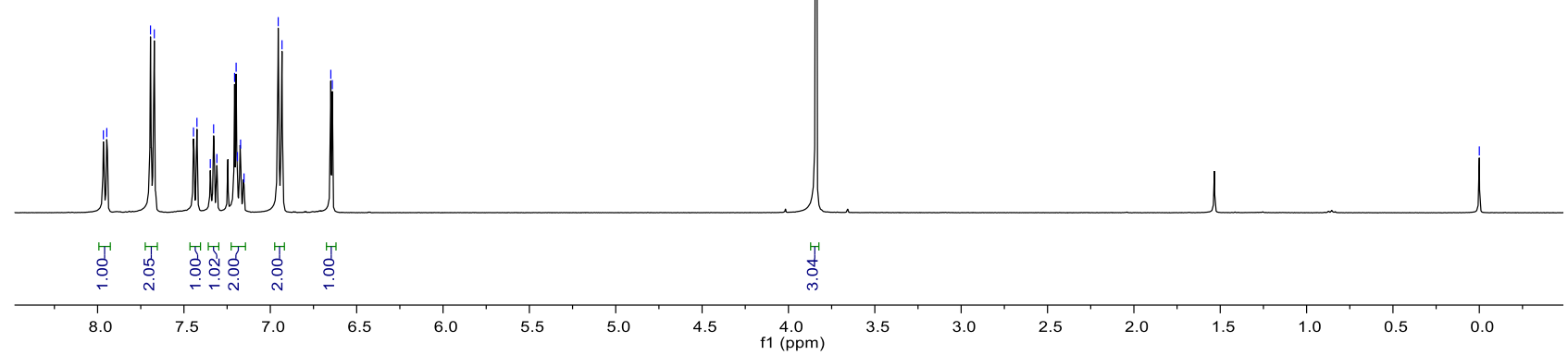

|
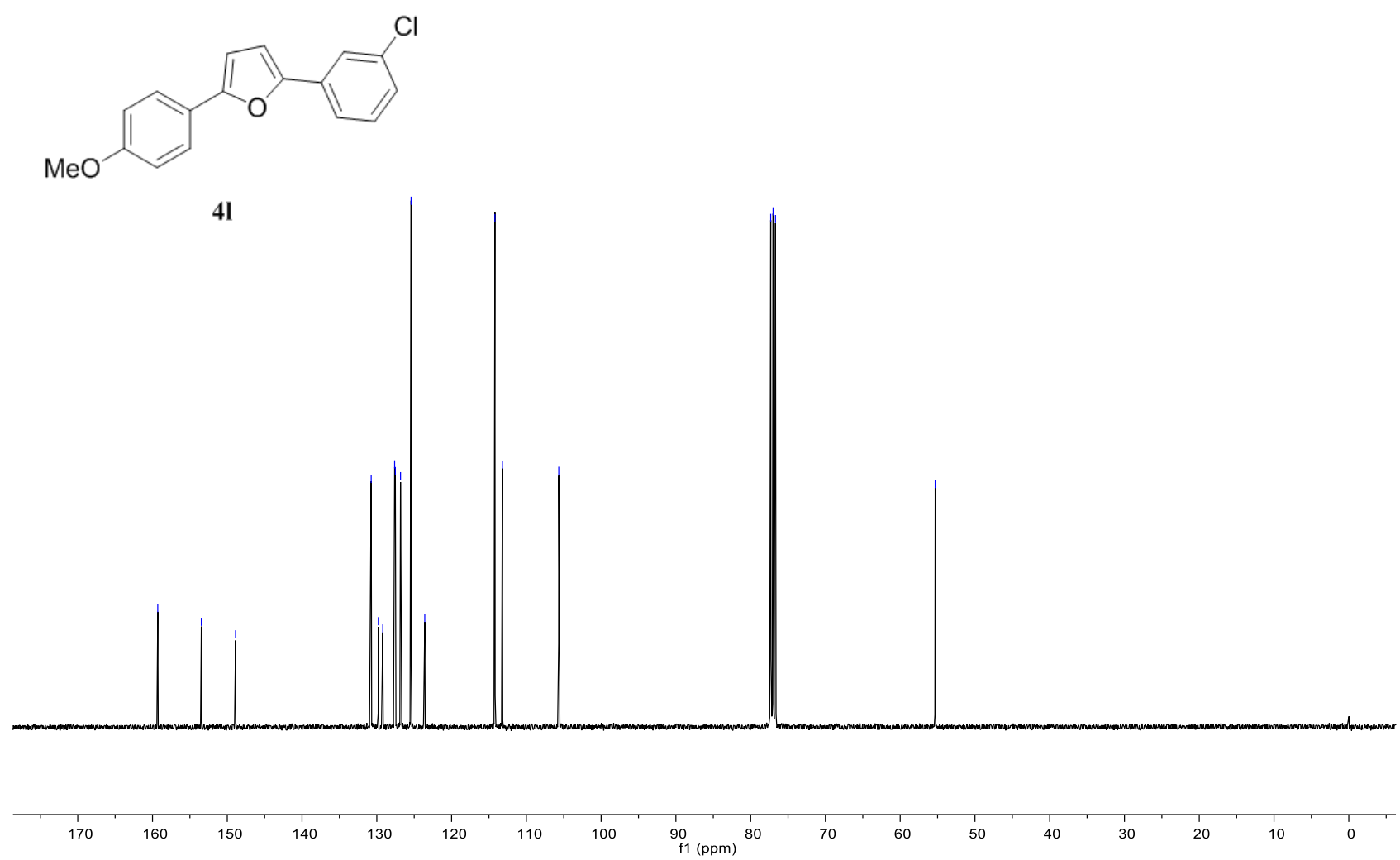


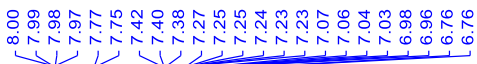

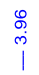

$\stackrel{\circ}{i}$
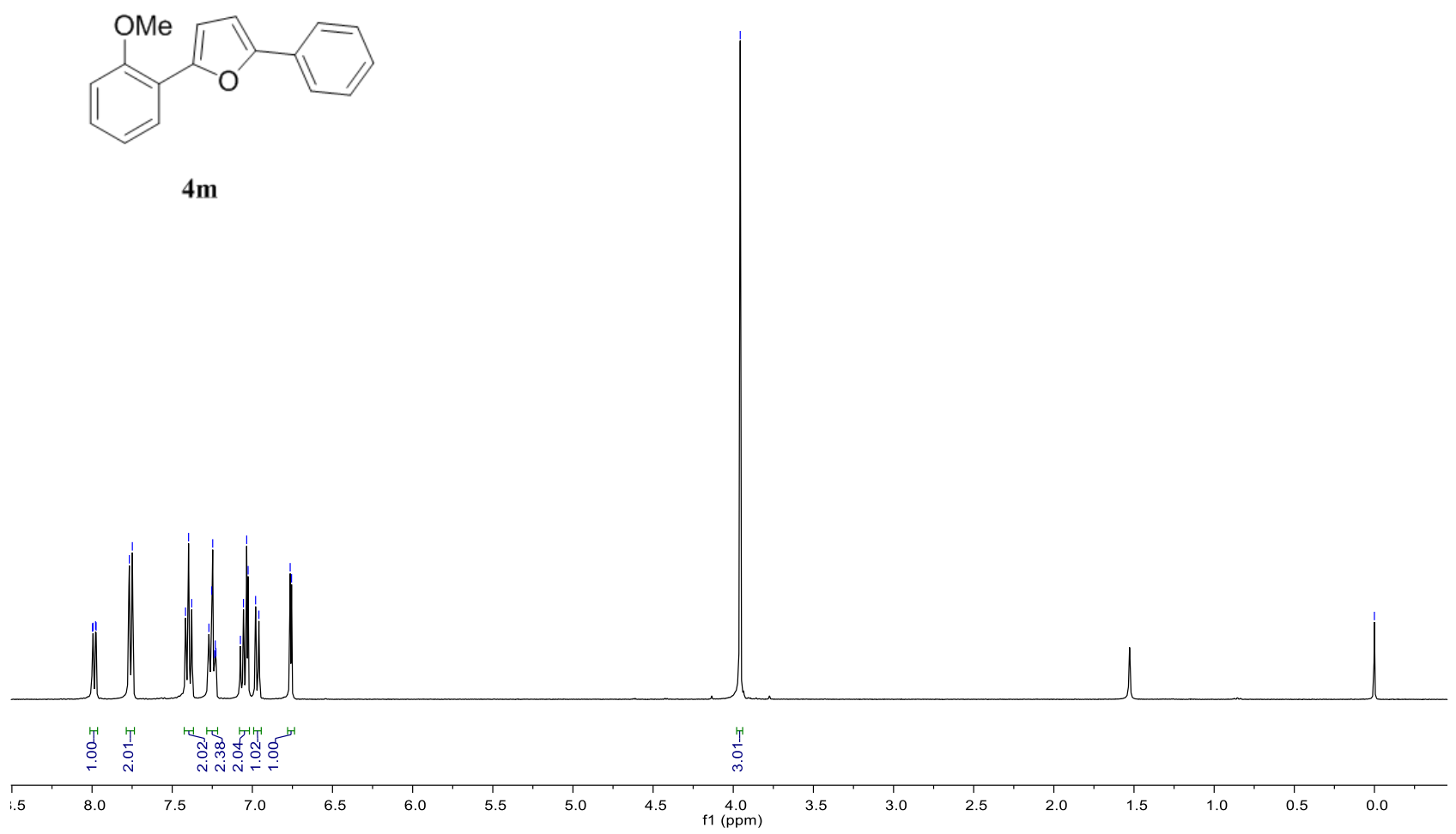

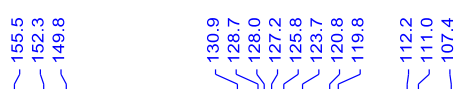

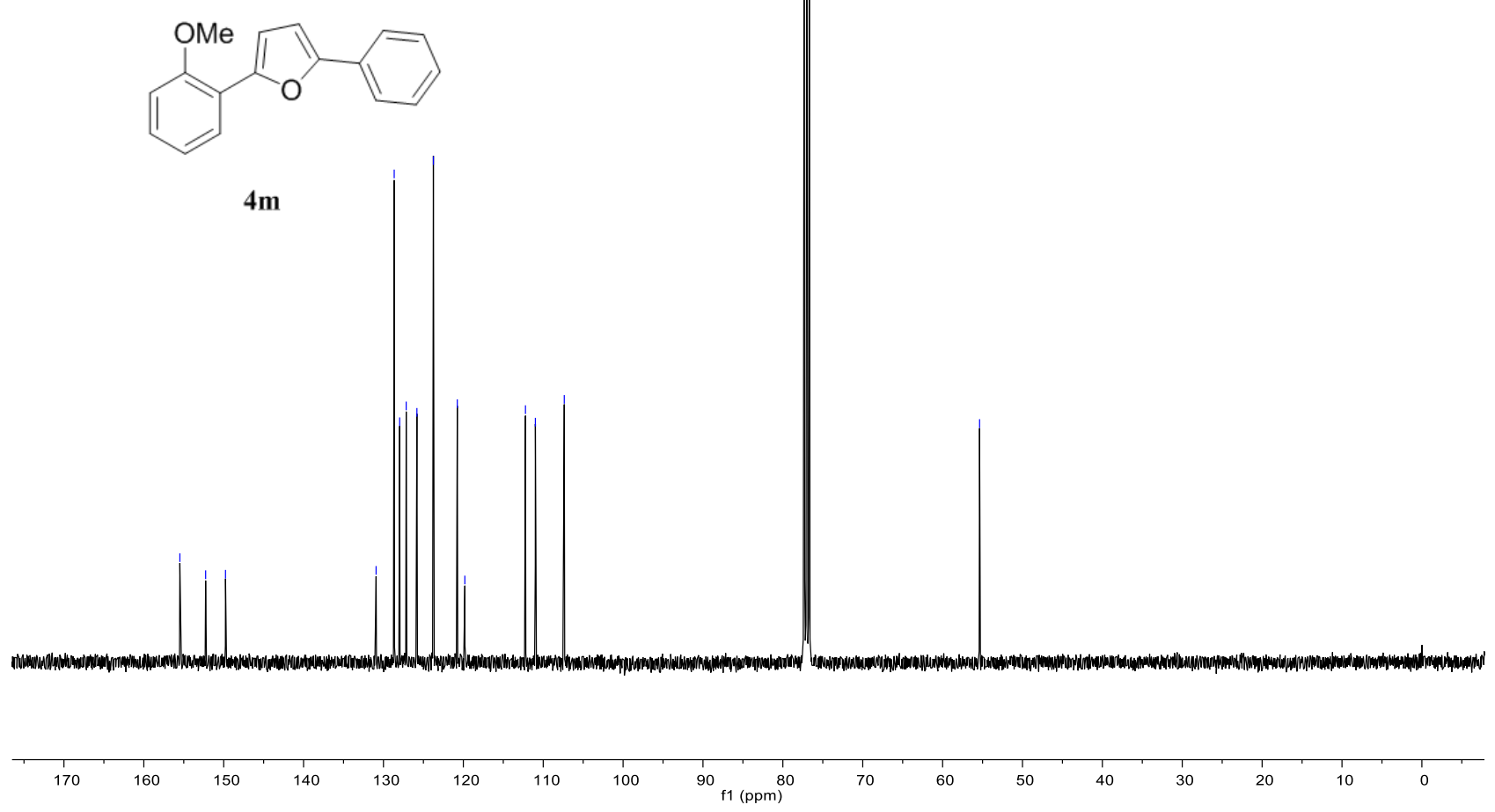

S53 


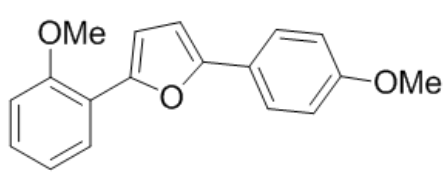

$4 n$

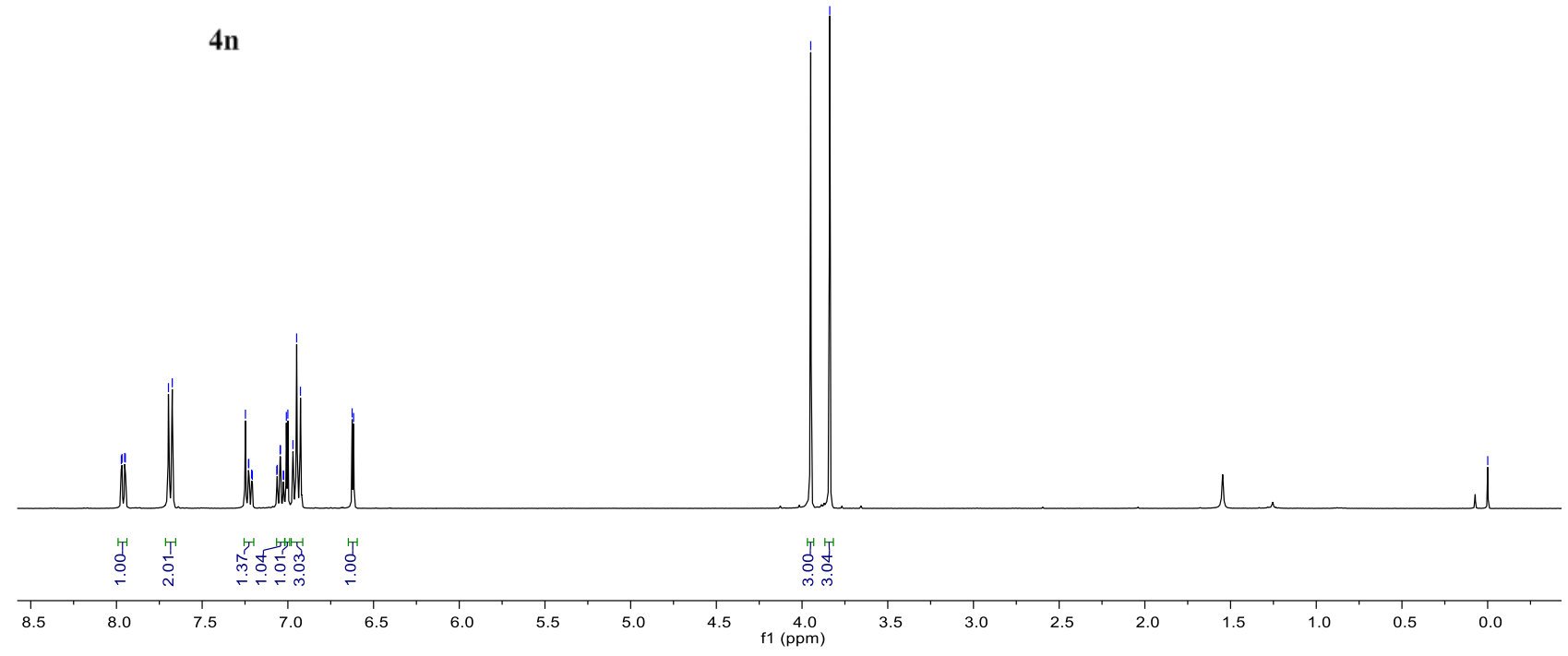

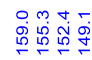

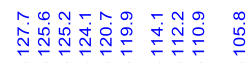

रोiा i

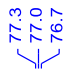

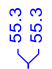

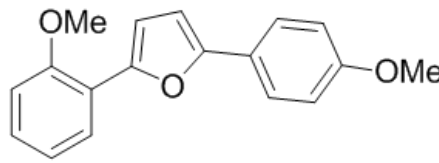

$4 n$

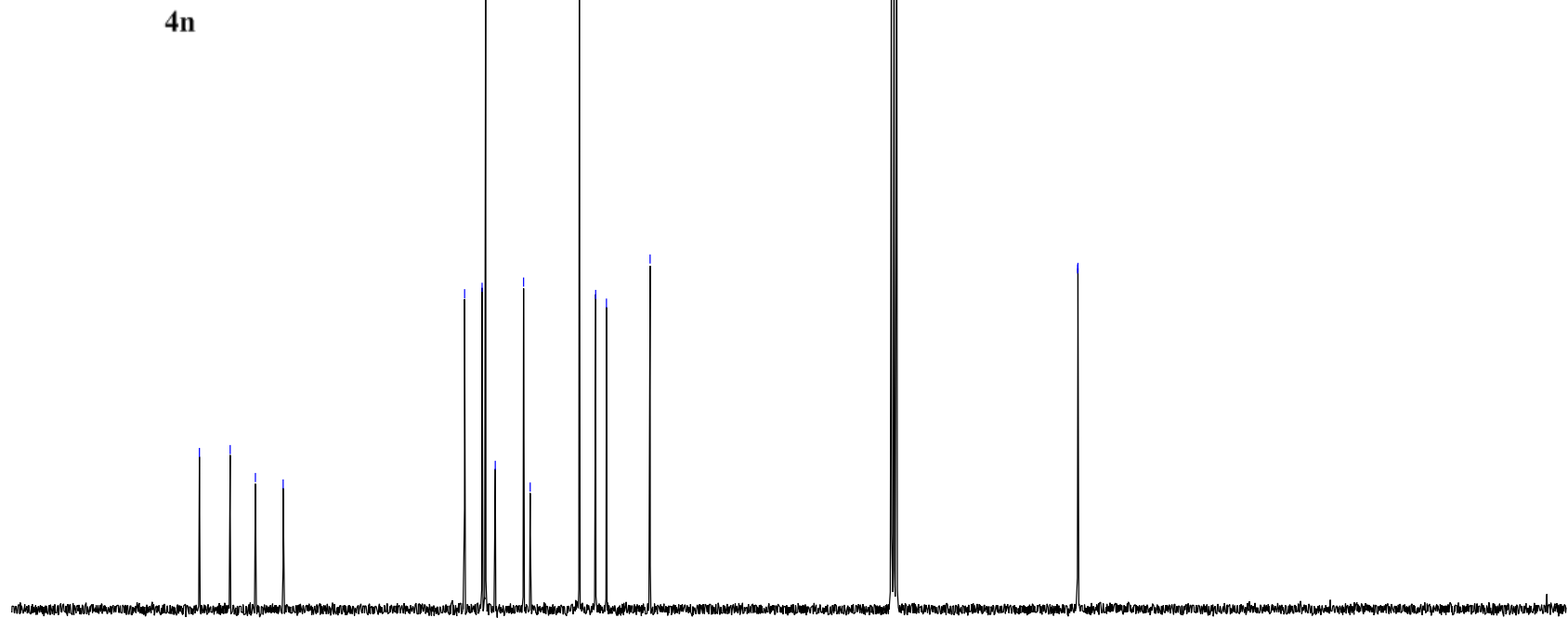

180

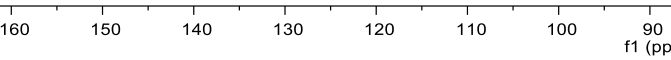

$\stackrel{90}{\mathrm{f} 1}(\mathrm{ppm})$

$80 \quad 70,60$

50

\begin{tabular}{l|l|l|l}
1 & 1 & 1 & 1
\end{tabular} 


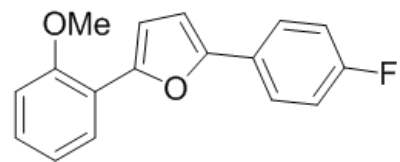

40

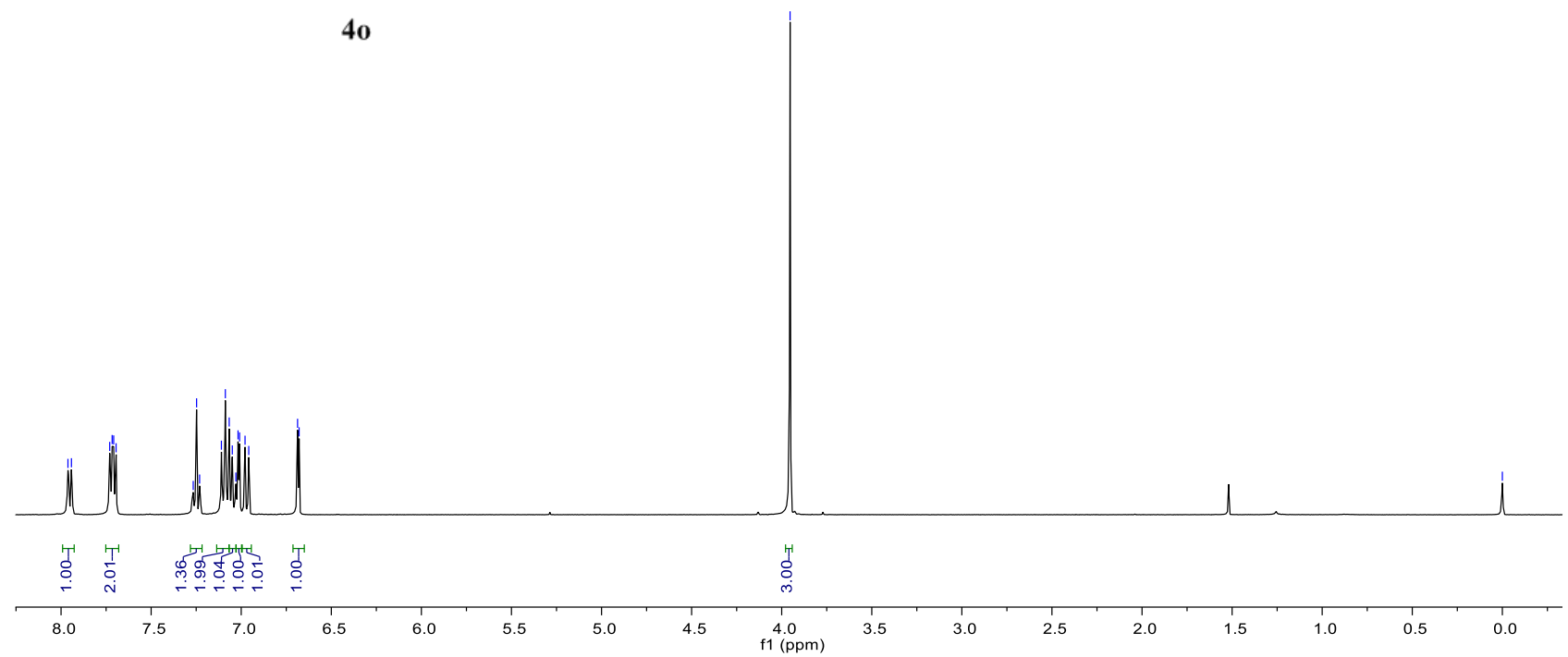

$\underbrace{1}_{1}$<smiles>COc1ccccc1-c1ccc(-c2ccc(F)cc2)o1</smiles>

40

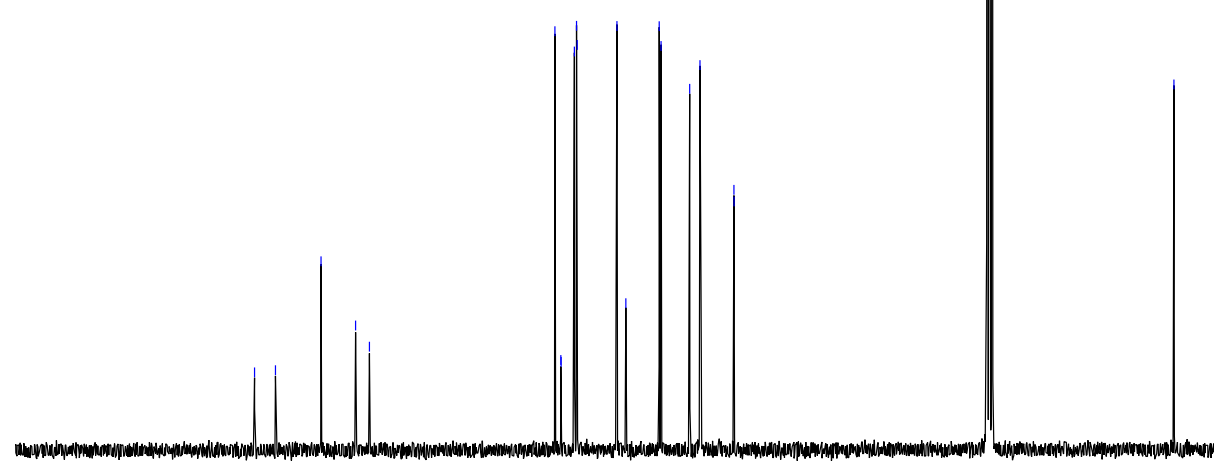

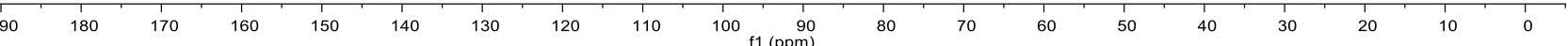



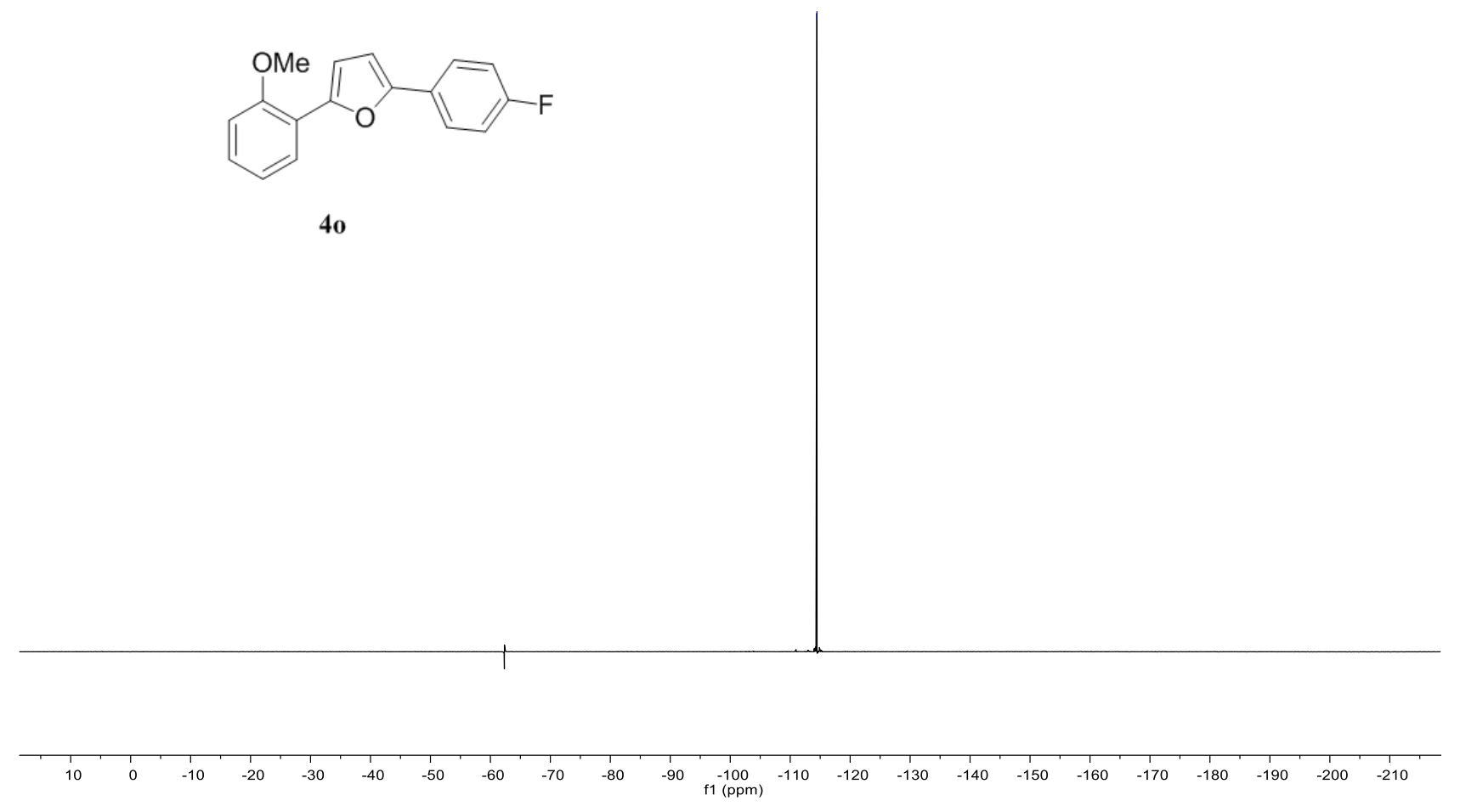

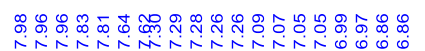

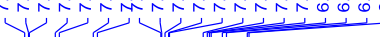

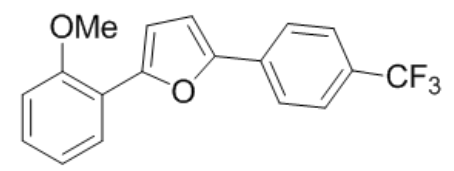

$4 p$

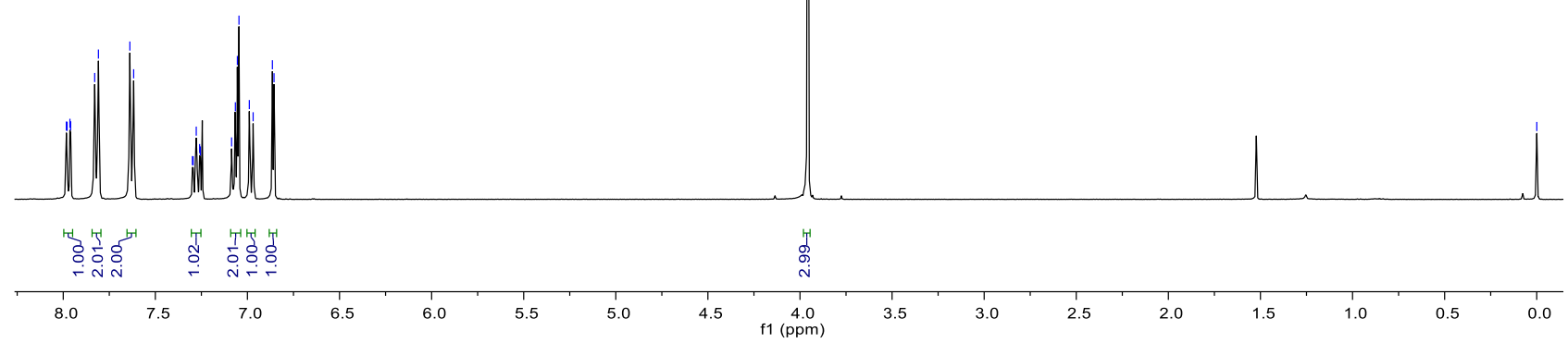



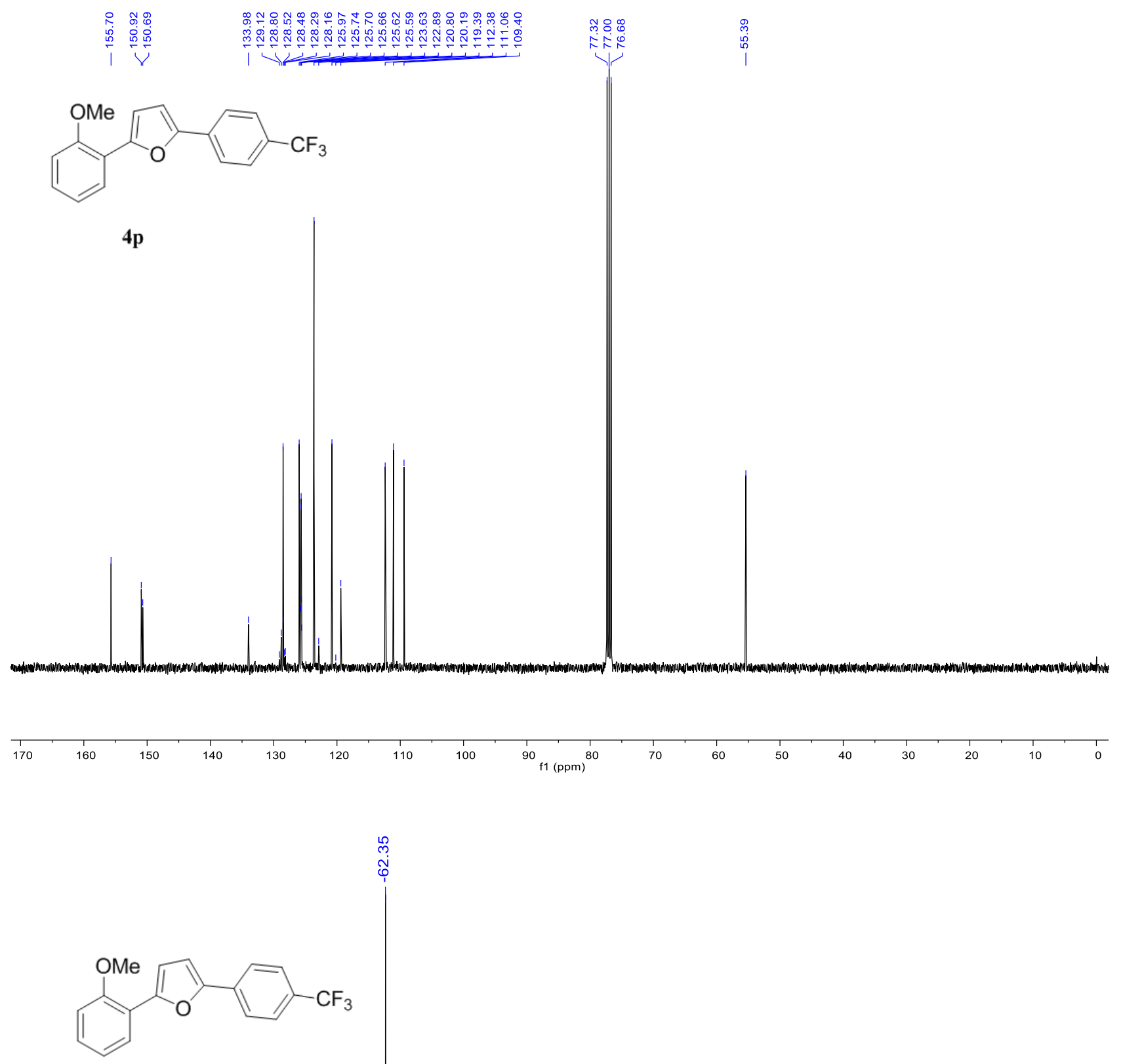

$4 p$ 

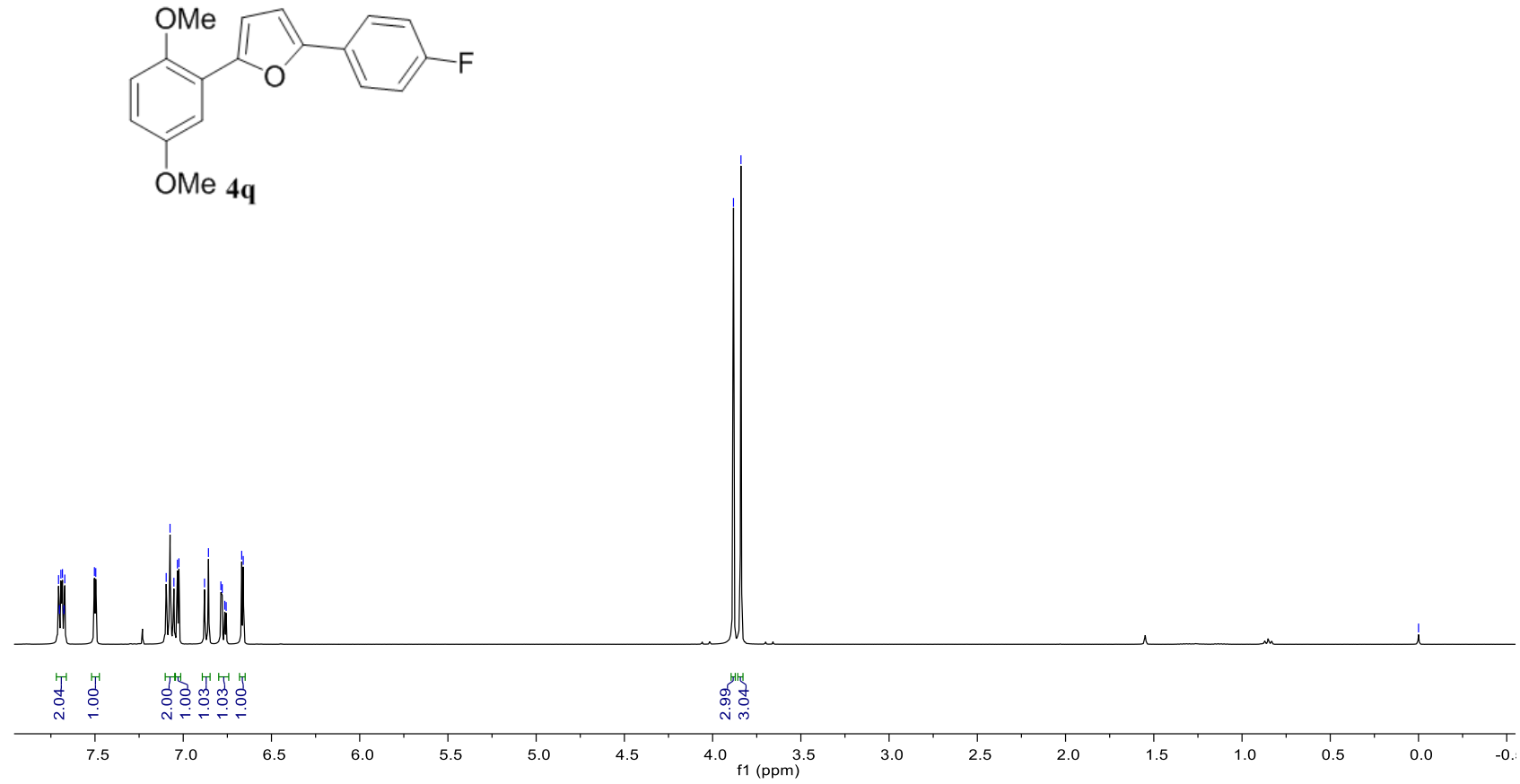

|

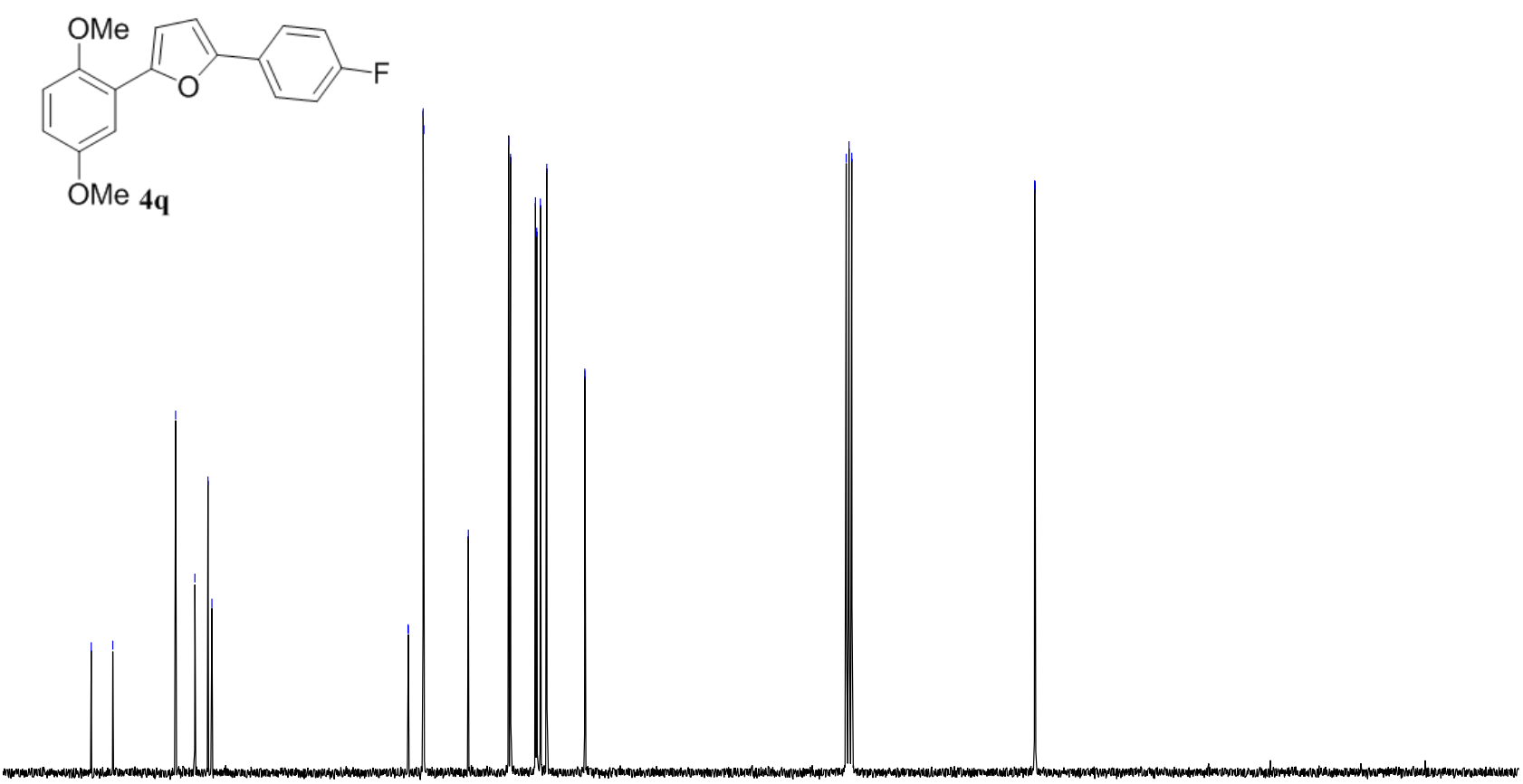

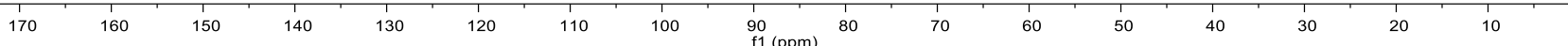




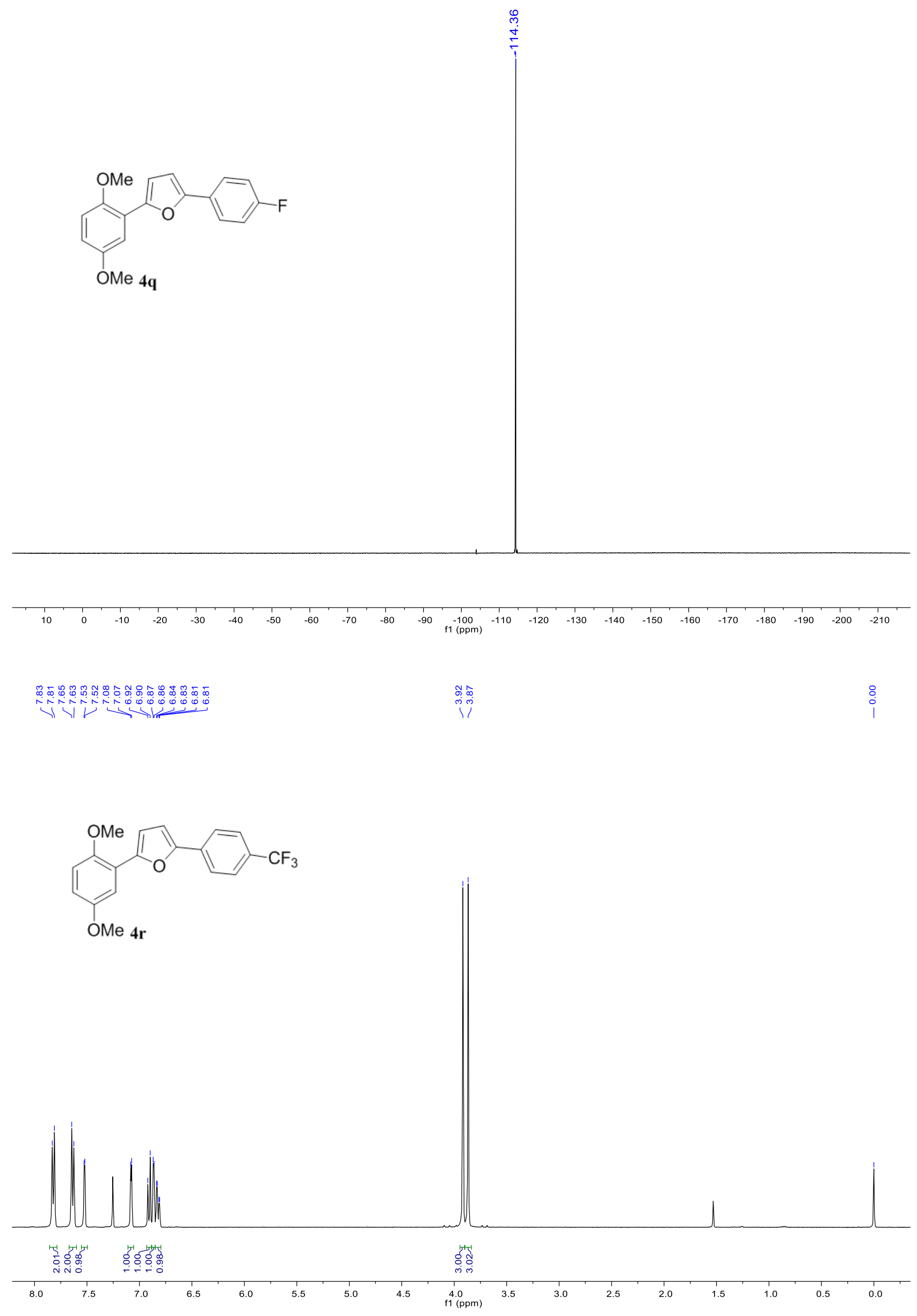



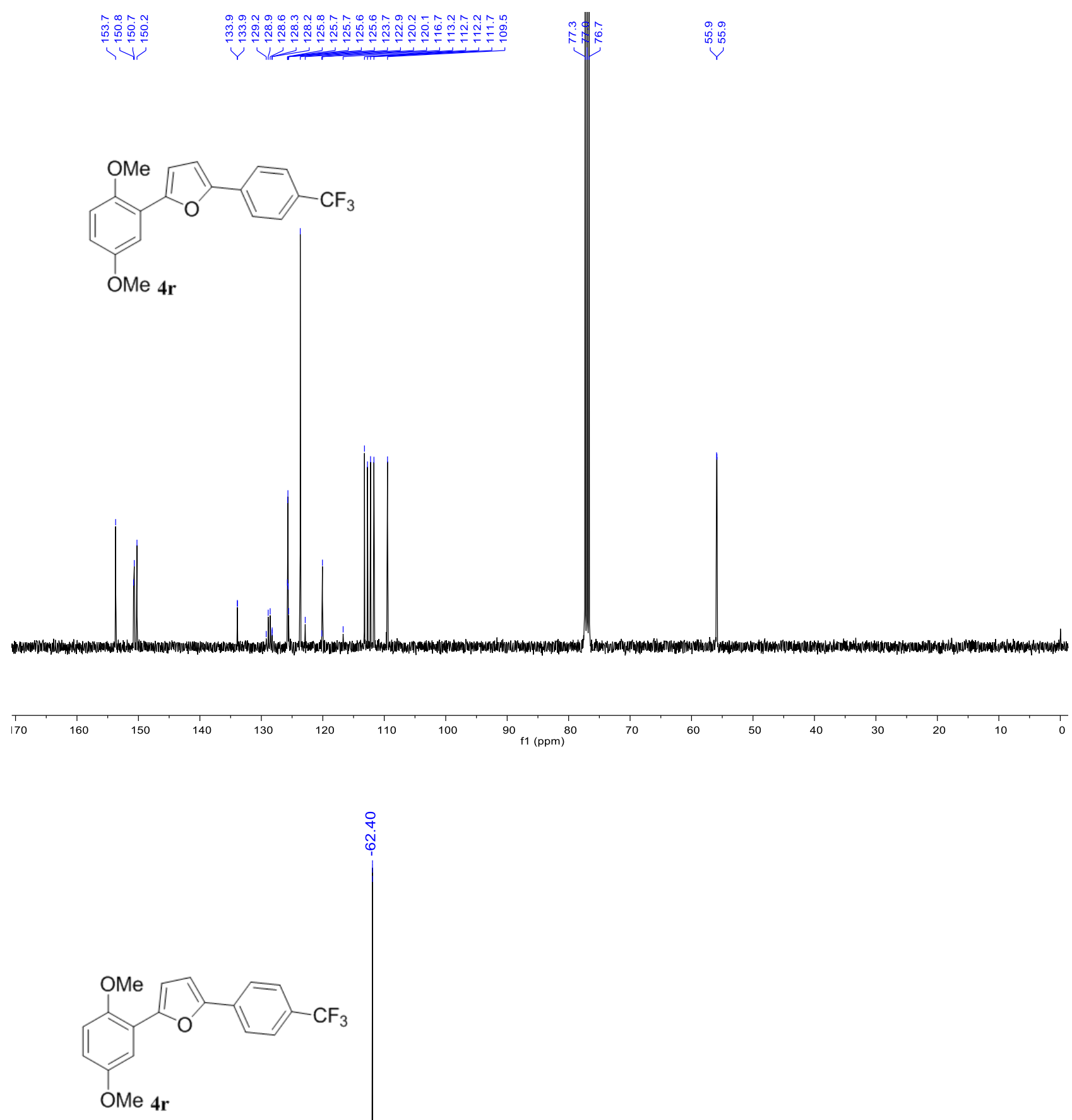

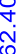

$\begin{array}{llllllllllllllllllllllll}10 & 0 & -10 & -20 & -30 & -40 & -50 & -60 & -70 & -80 & -90 & -100 & -110 & -120 & -130 & -140 & -150 & -160 & -170 & -180 & -190 & -200 & -210 & \end{array}$ 


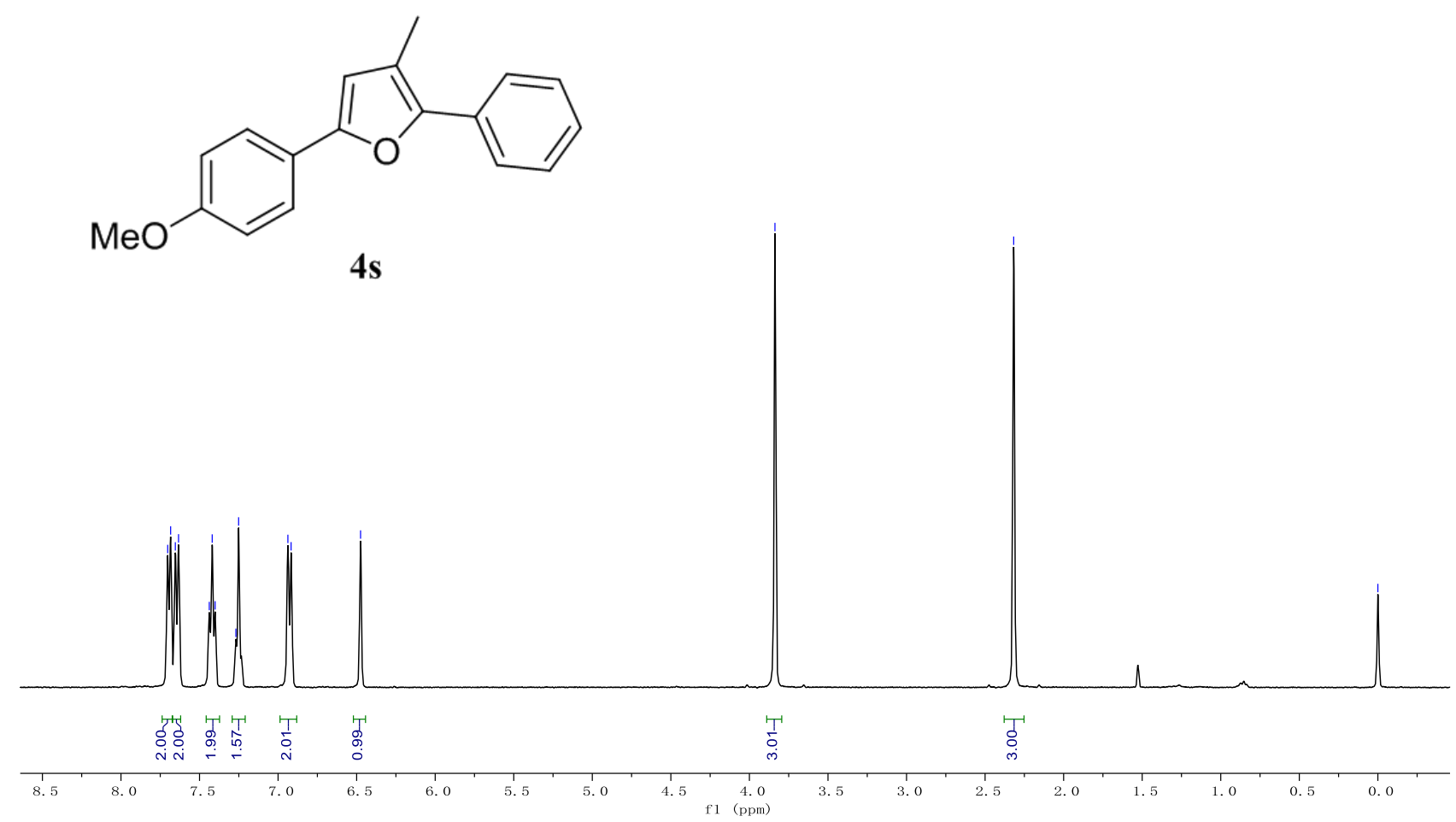

$\overbrace{\substack{1 \\ 0}}^{1}$<smiles>COc1ccc(-c2cc(C)c(-c3ccccc3)o2)cc1</smiles>

$4 s$

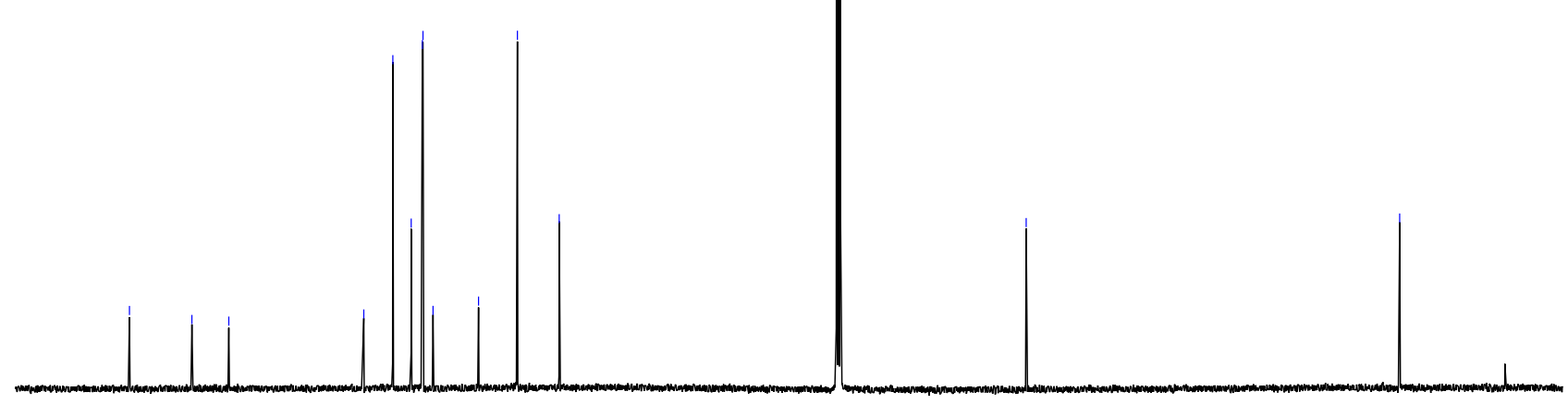

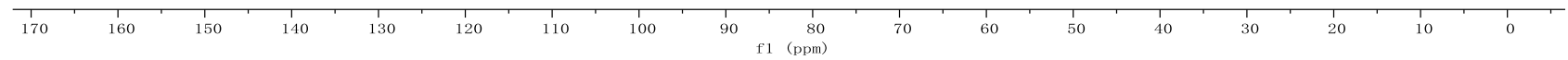




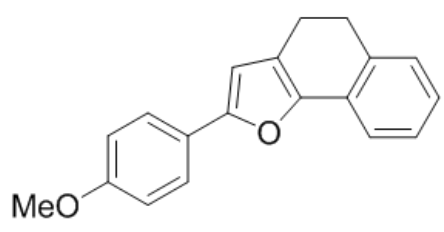

$4 t$

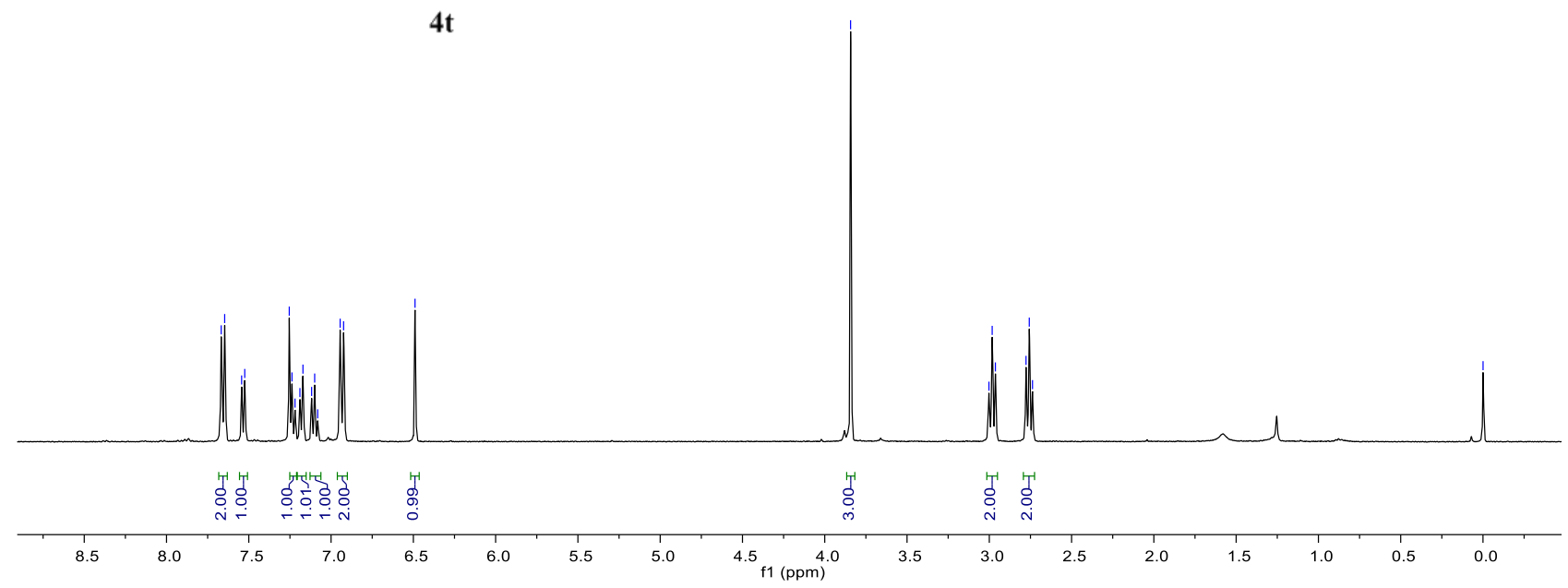

إl

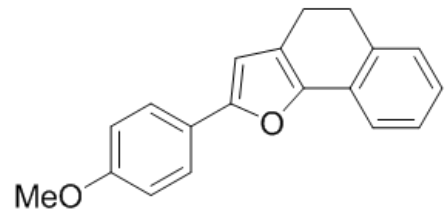

$4 \mathrm{t}$

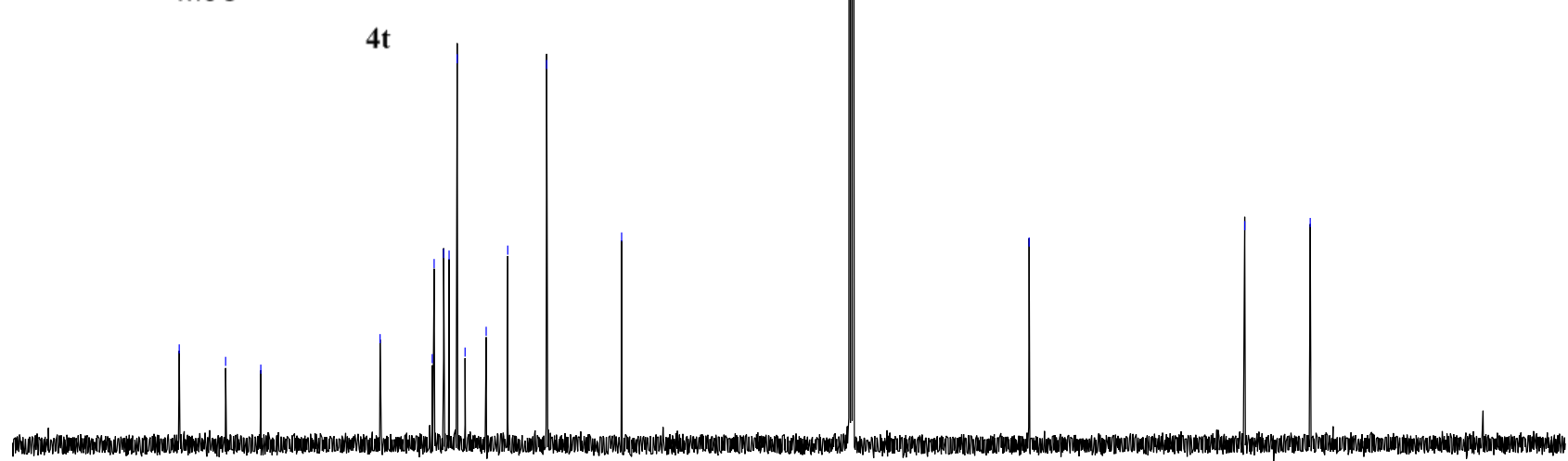

170 

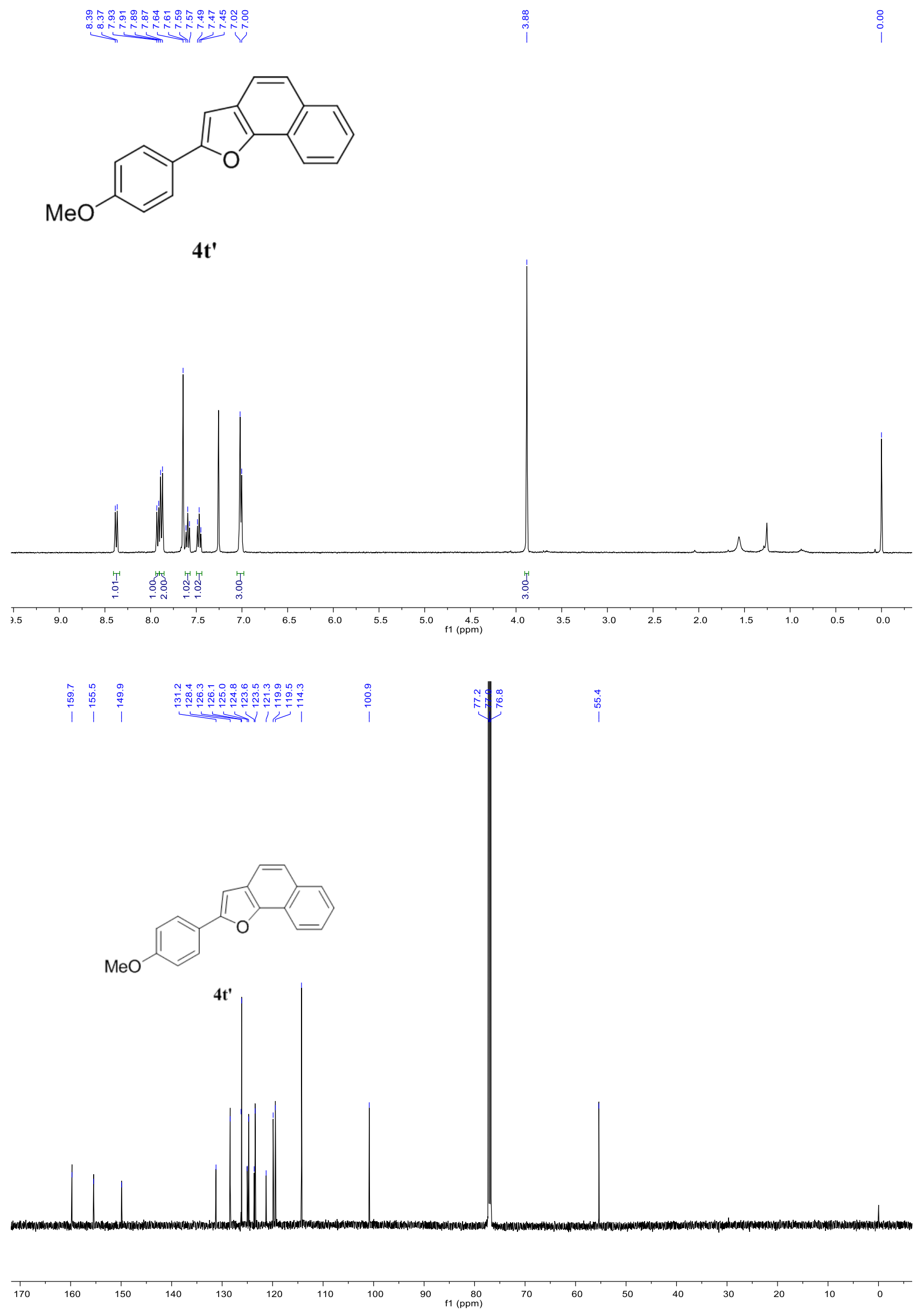


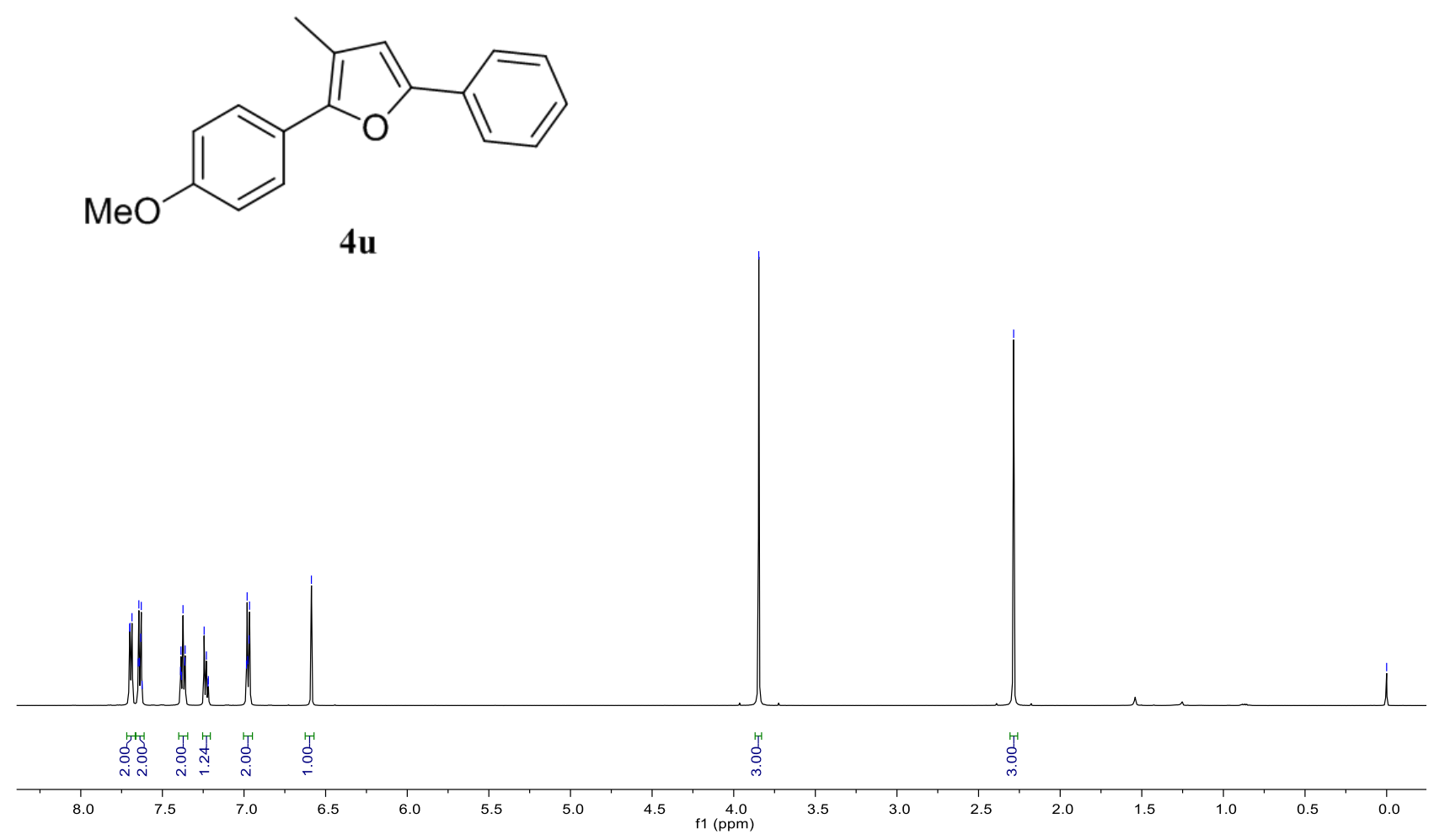

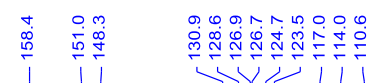

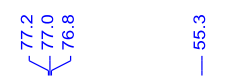<smiles>COc1ccc(-c2oc(-c3ccccc3)cc2C)cc1</smiles> 

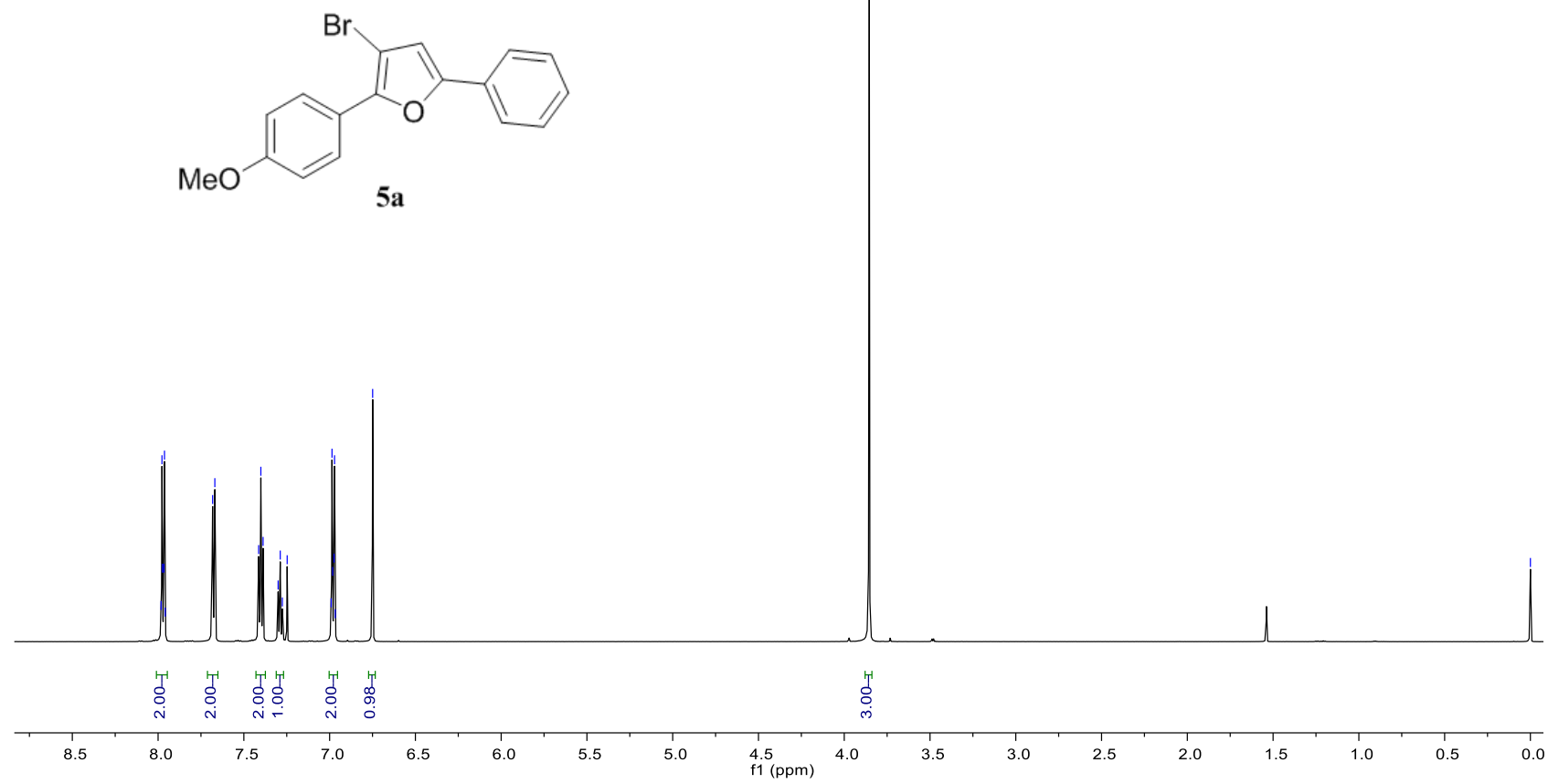

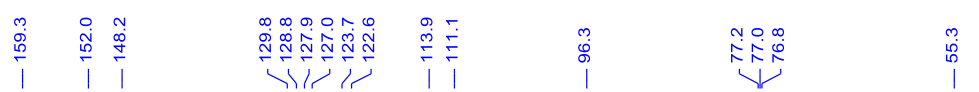

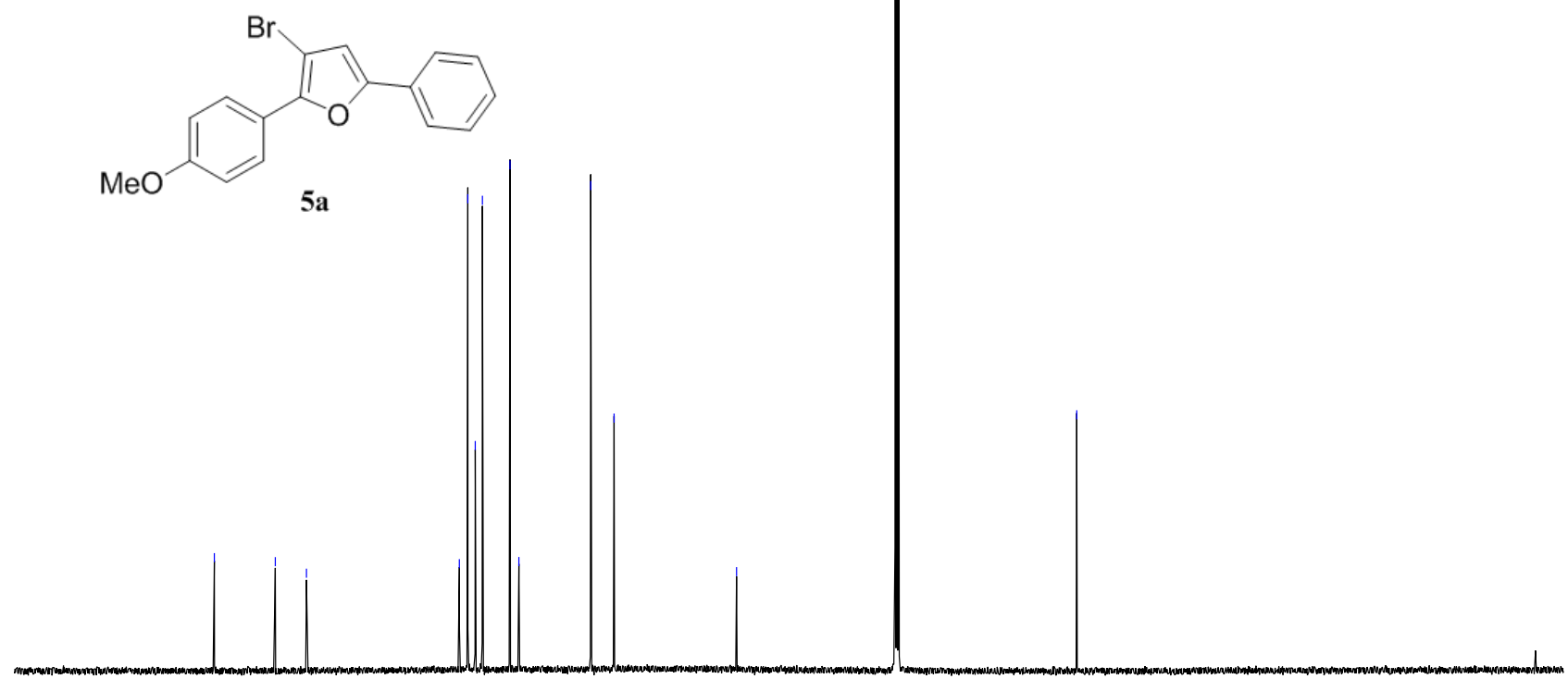

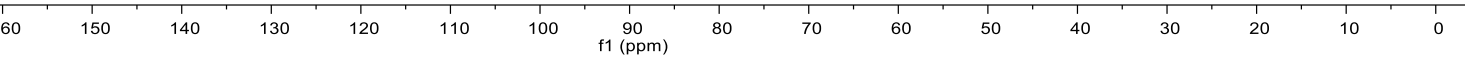


<smiles>COc1ccc(-c2oc(-c3ccc(OC)cc3)c(Br)c2Br)cc1</smiles>

$5 c$

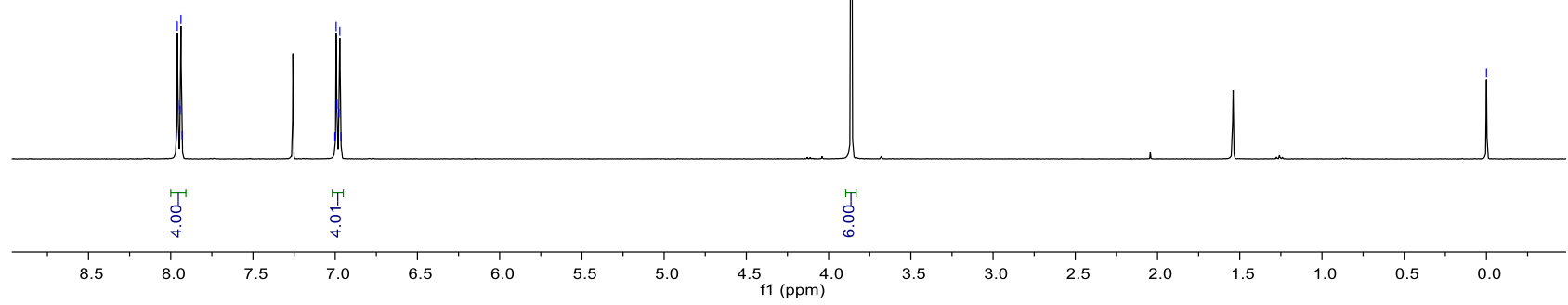

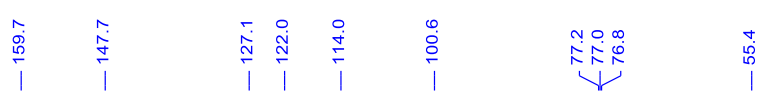<smiles>COc1ccc(-c2oc(-c3ccc(OC)cc3)c(Br)c2Br)cc1</smiles>

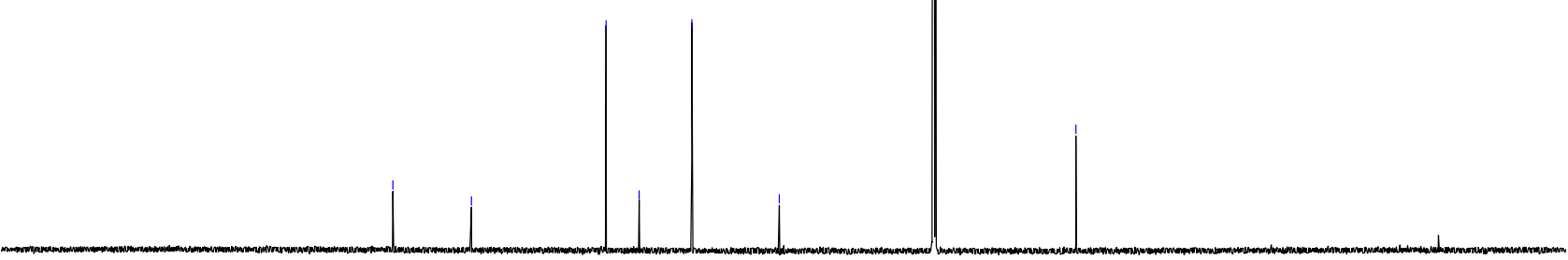

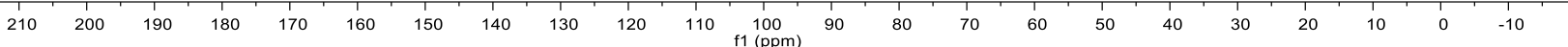




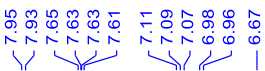
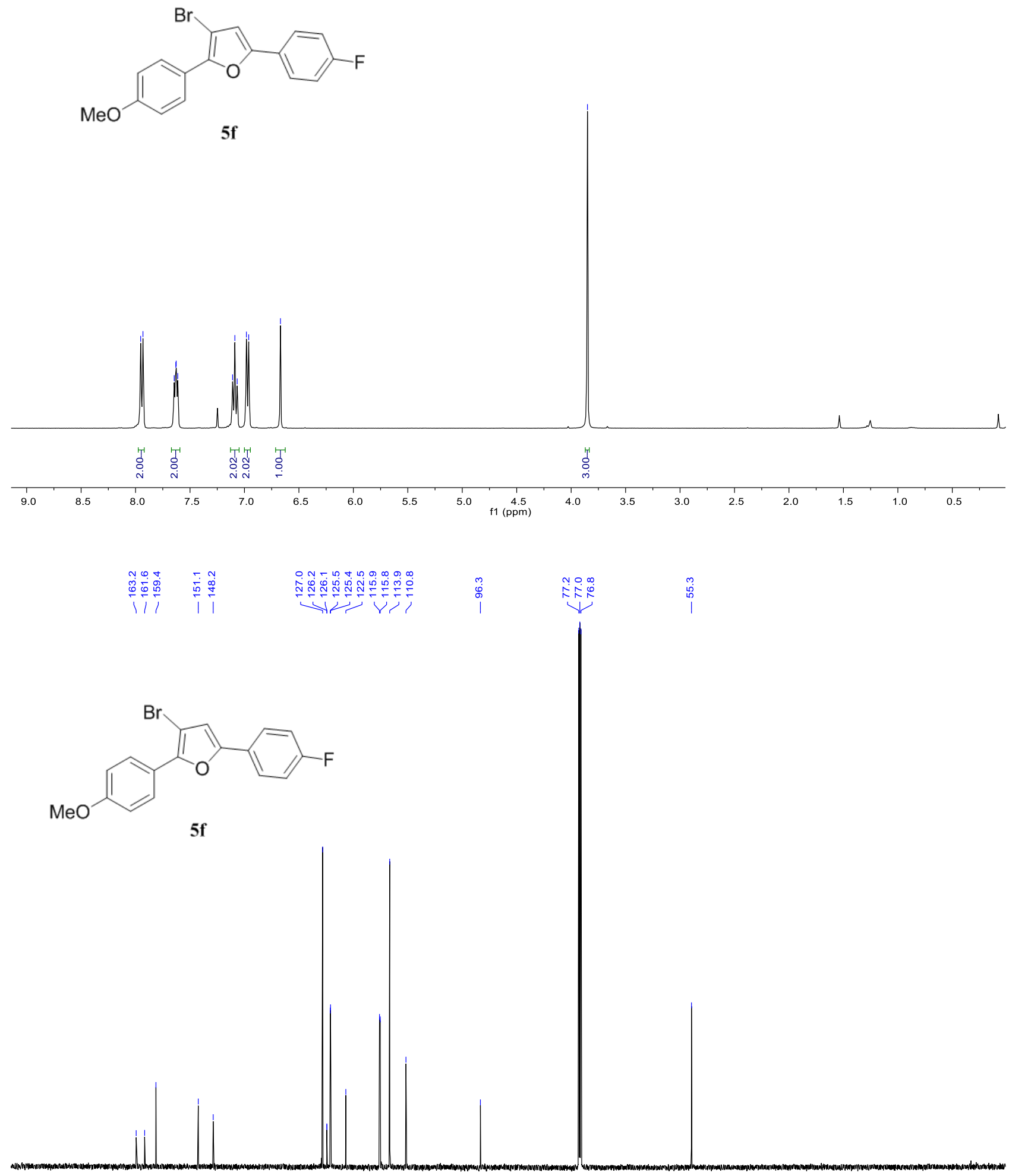

$\begin{array}{llllllllllllllllllll}180 & 170 & 160 & 150 & 140 & 130 & 120 & 110 & 100 & 90 & 80 & 70 & 60 & 50 & 40 & 30 & 20 & 10 & 0\end{array}$ 
<smiles>COc1ccc(-c2oc(-c3ccc(F)cc3)cc2Br)cc1</smiles>
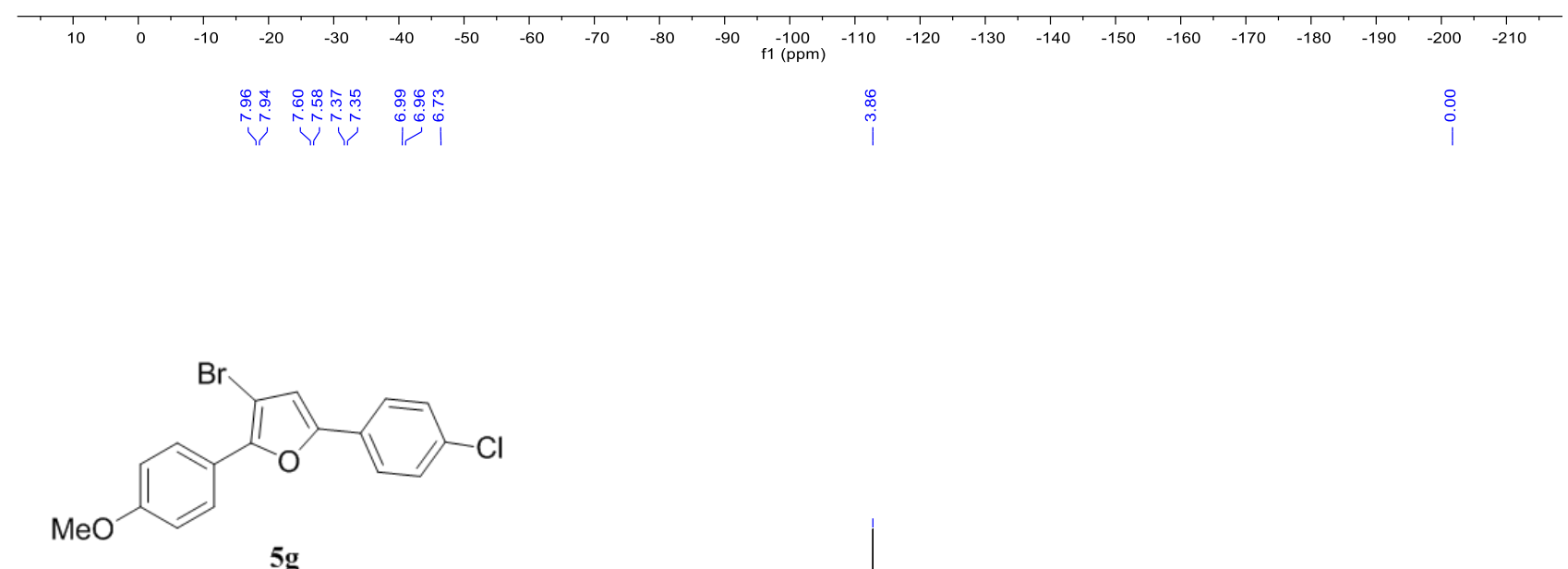

$5 g$

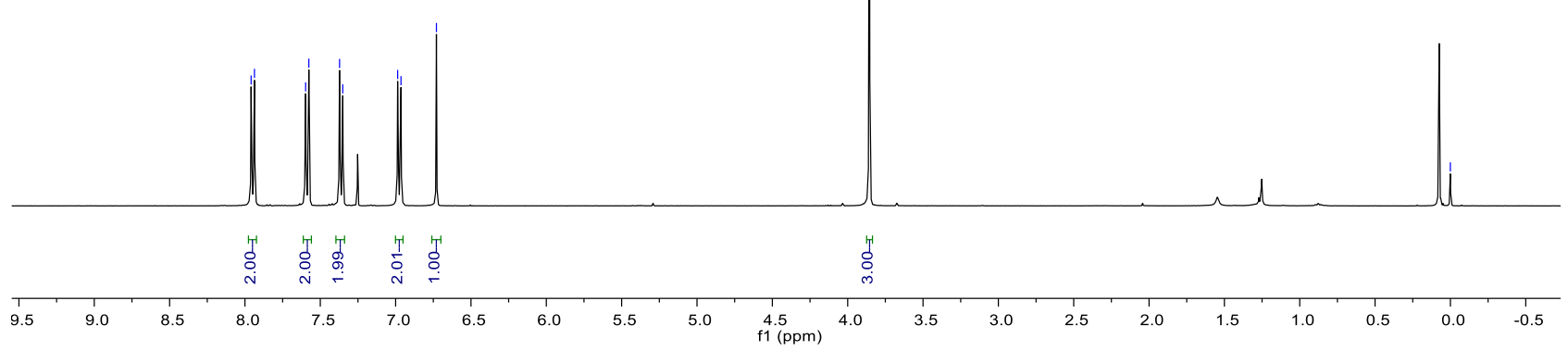



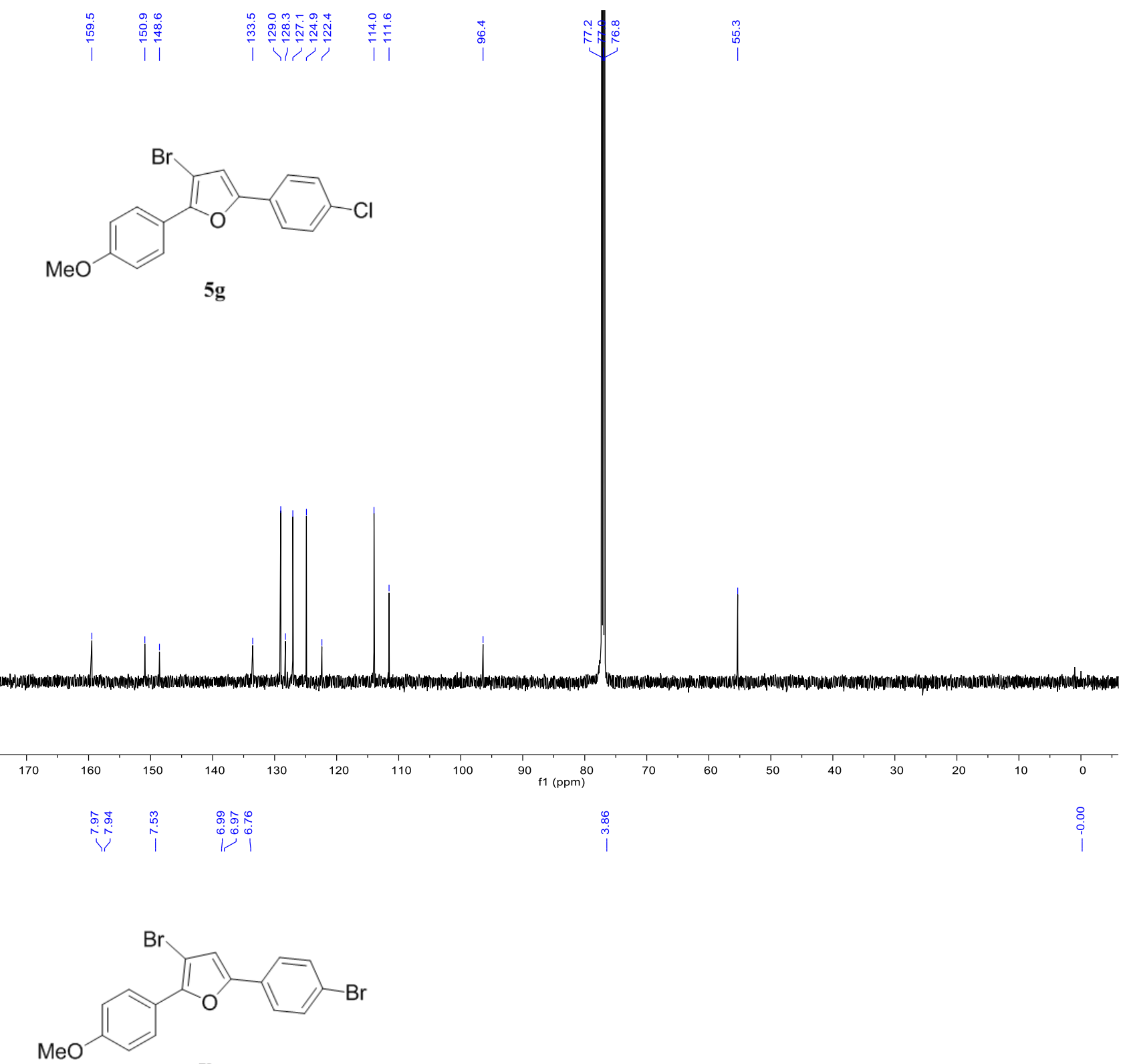

$5 h$

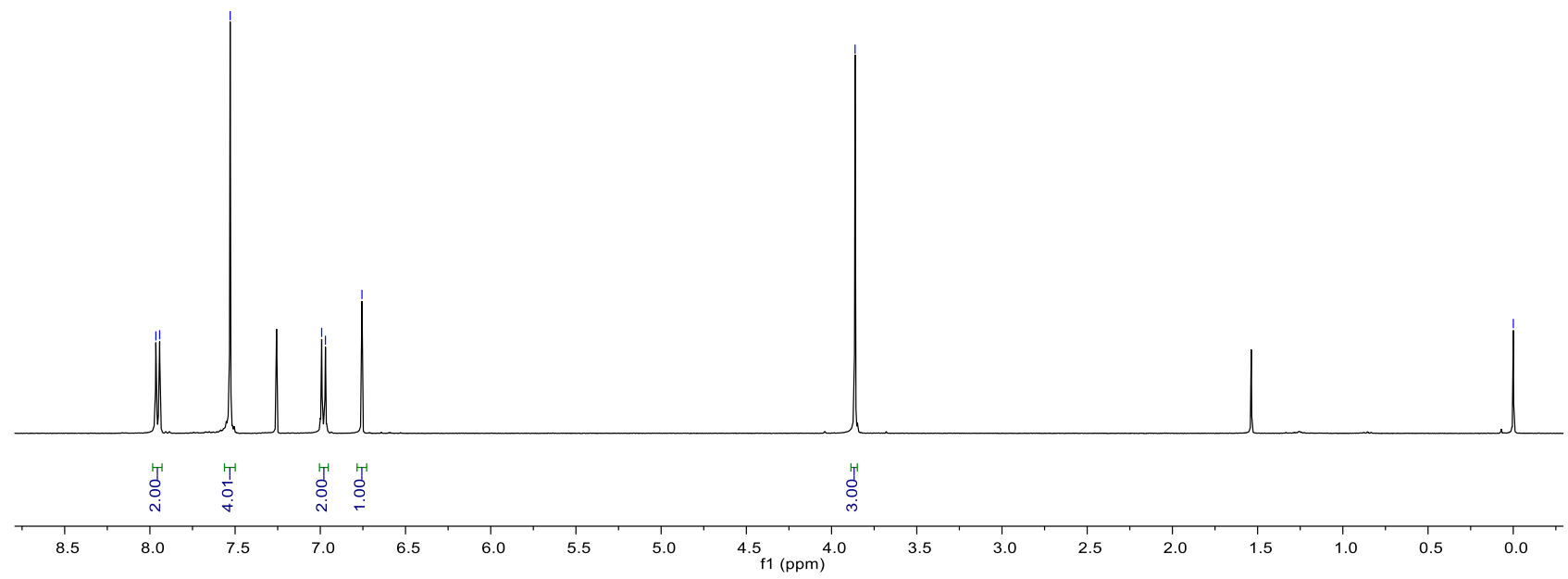




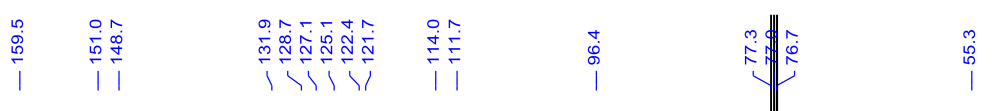<smiles>COc1ccc(-c2oc(-c3ccc(Br)cc3)cc2Br)cc1</smiles>

$5 \mathrm{~h}$

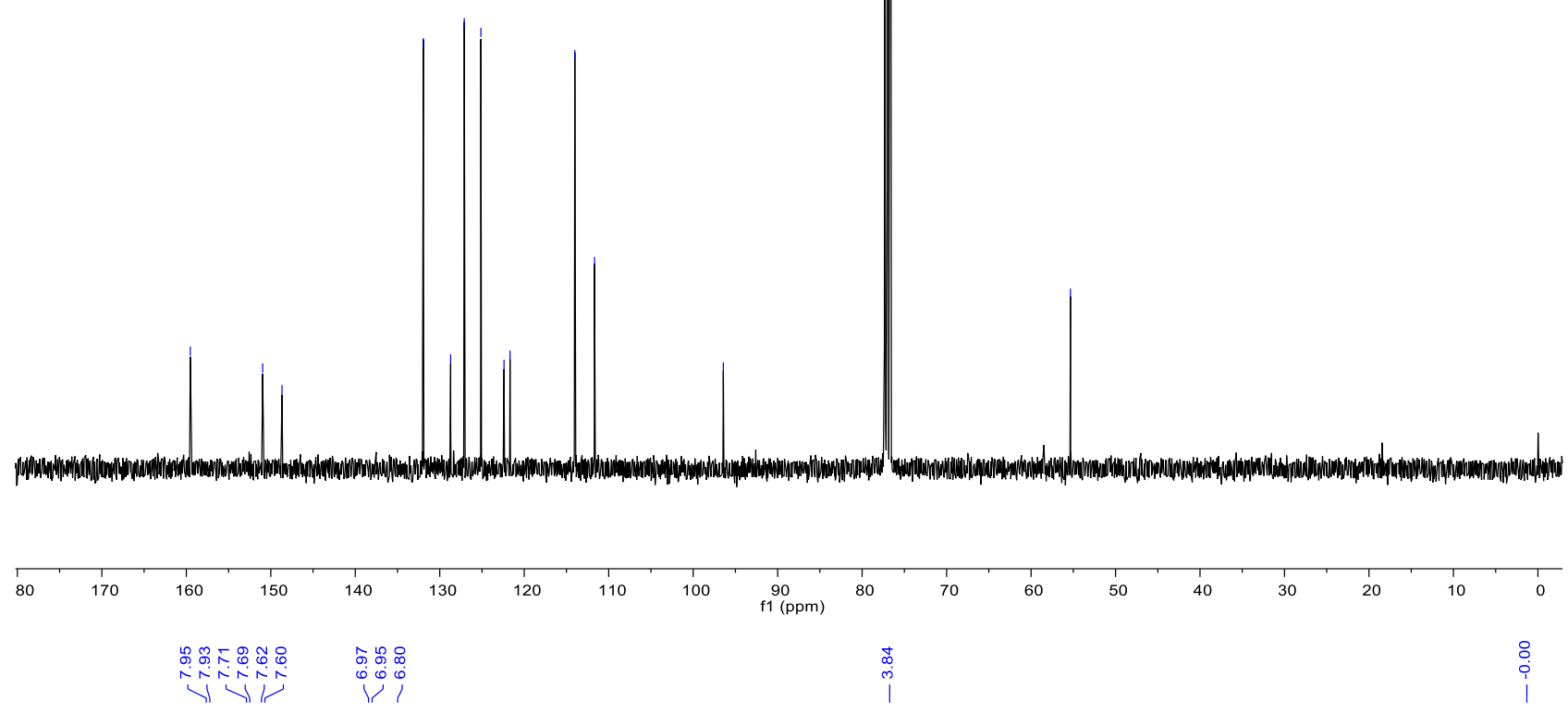<smiles>COc1ccc(-c2oc(-c3ccc(C(F)(F)F)cc3)cc2Br)cc1</smiles>

$5 i$
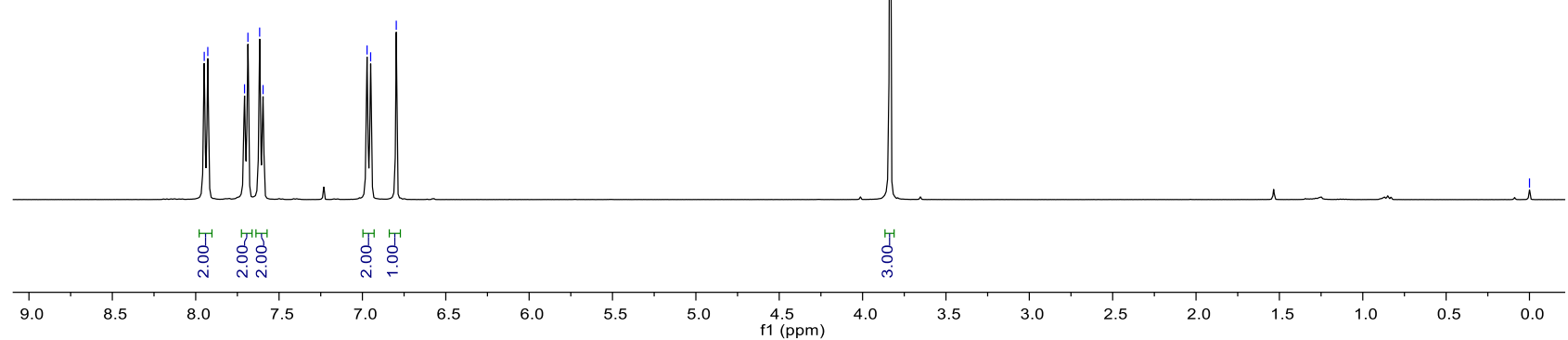

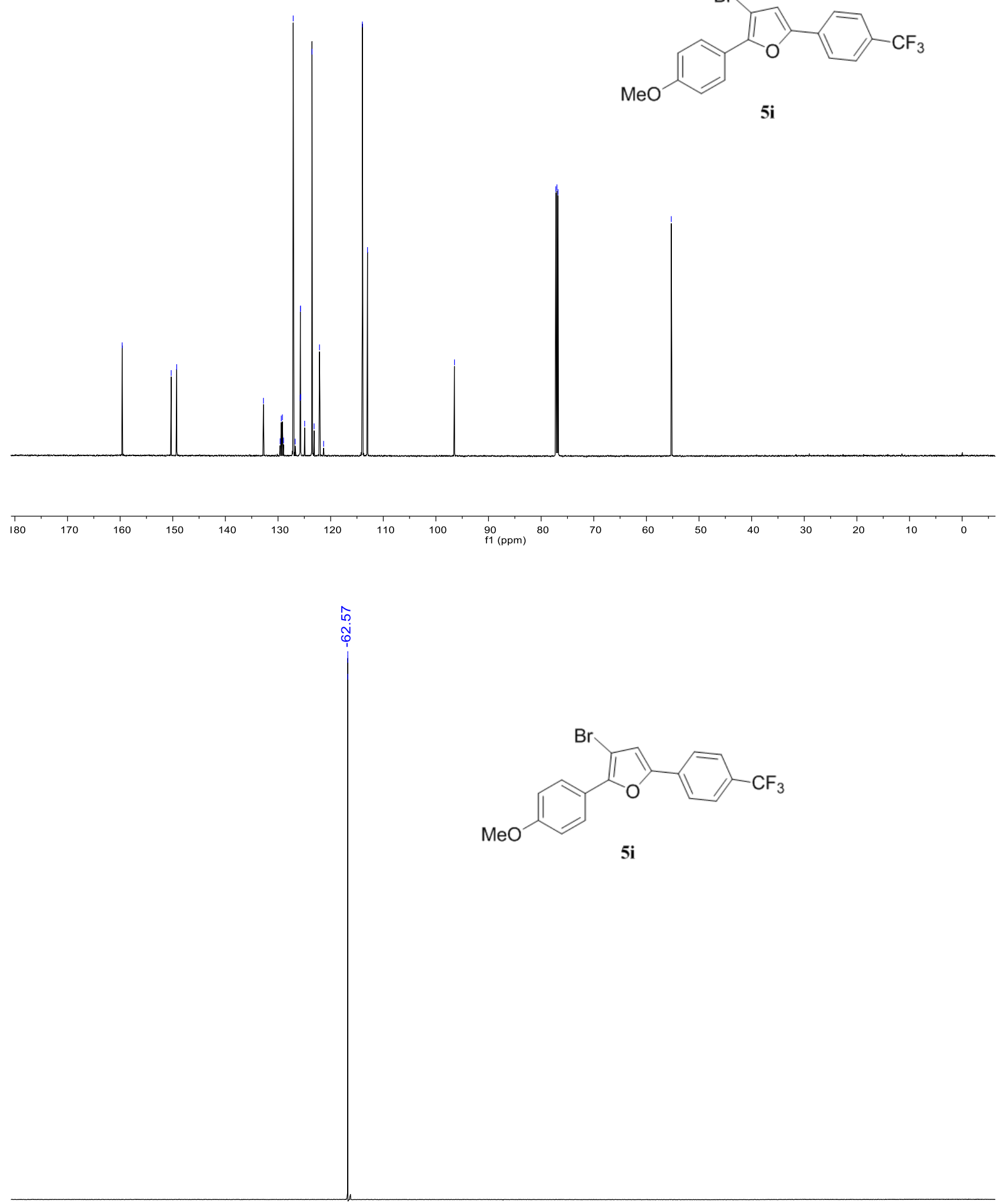

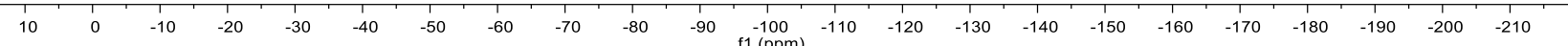




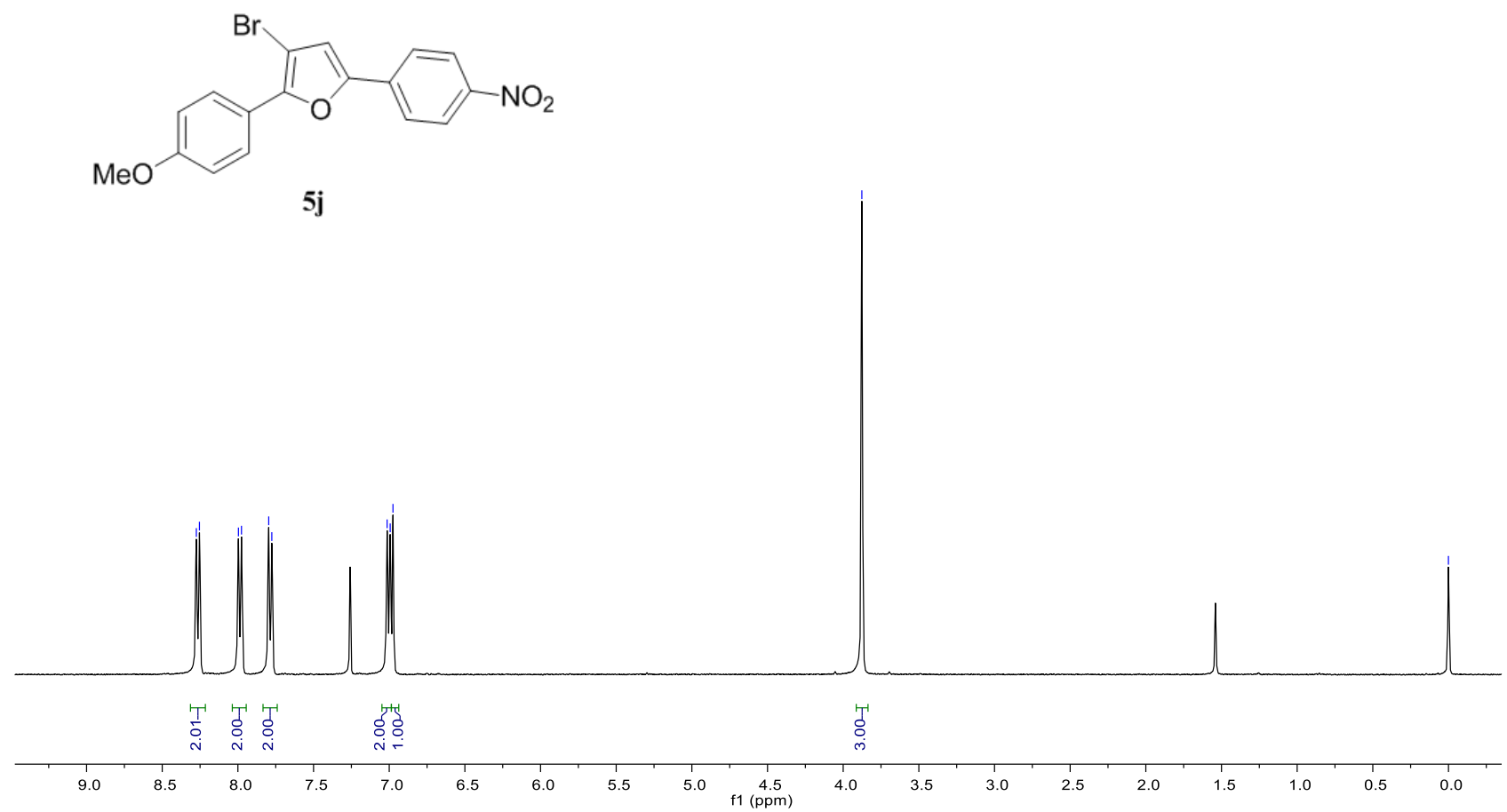
(1)

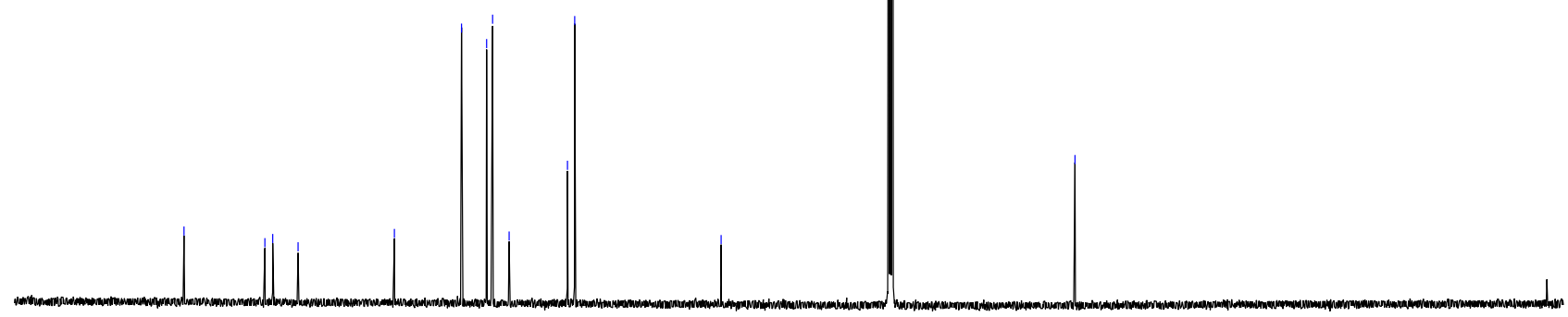

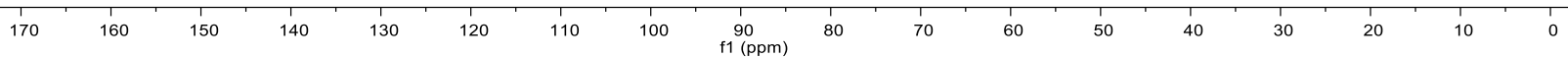



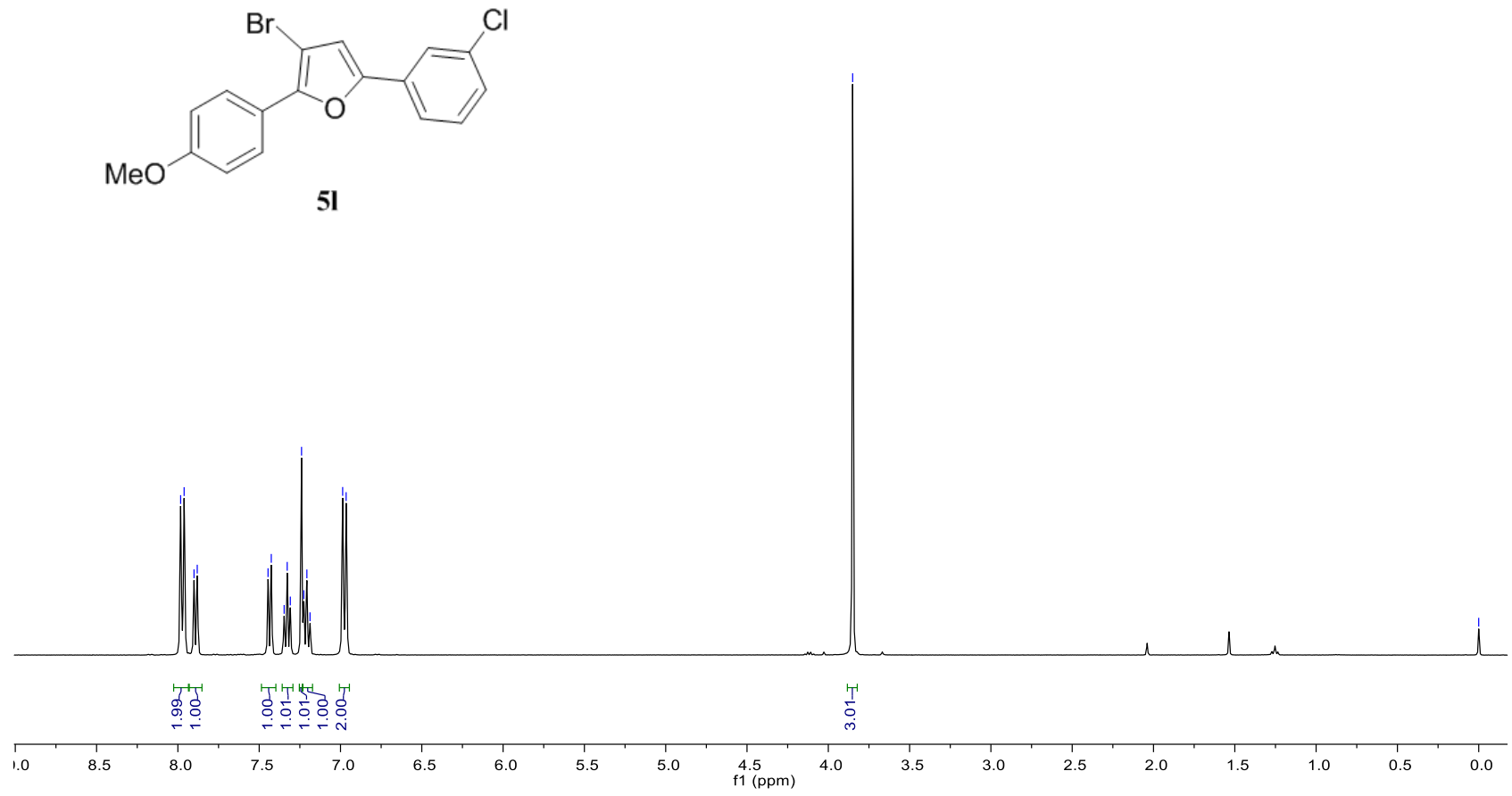

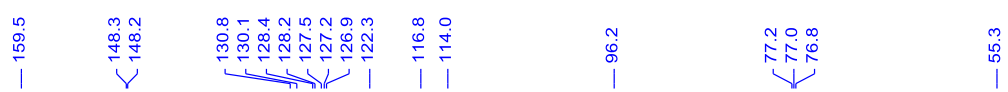
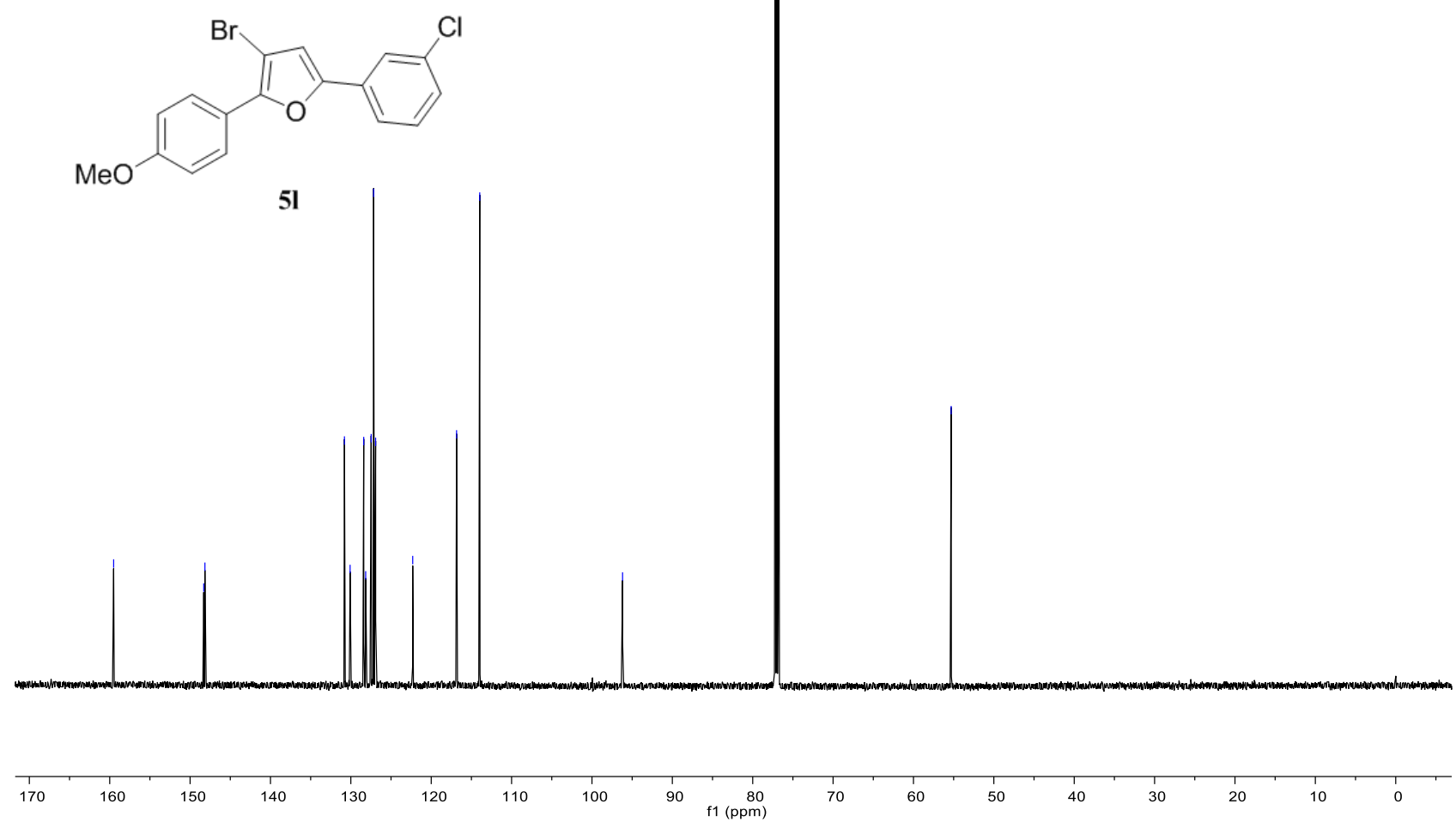


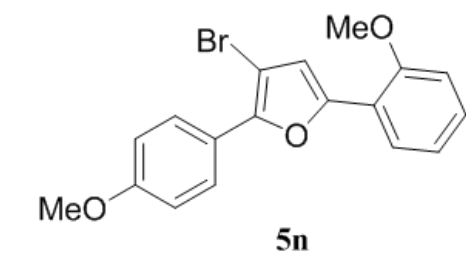

$5 n$

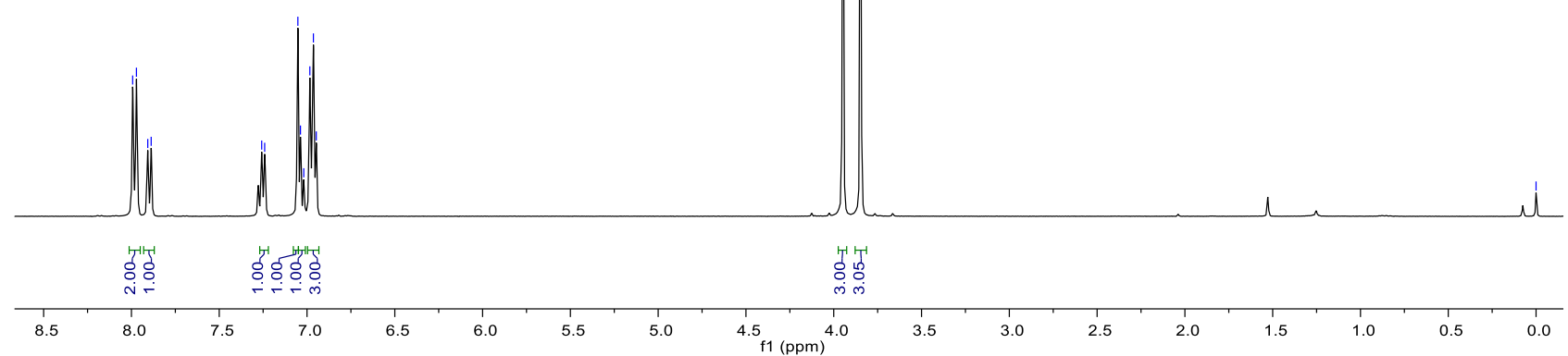

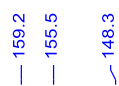

年

$\sqrt{\sqrt{2}}$

Y<smiles>COc1ccc(-c2oc(-c3ccccc3OC)cc2Br)cc1</smiles>

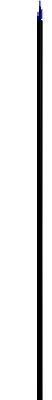

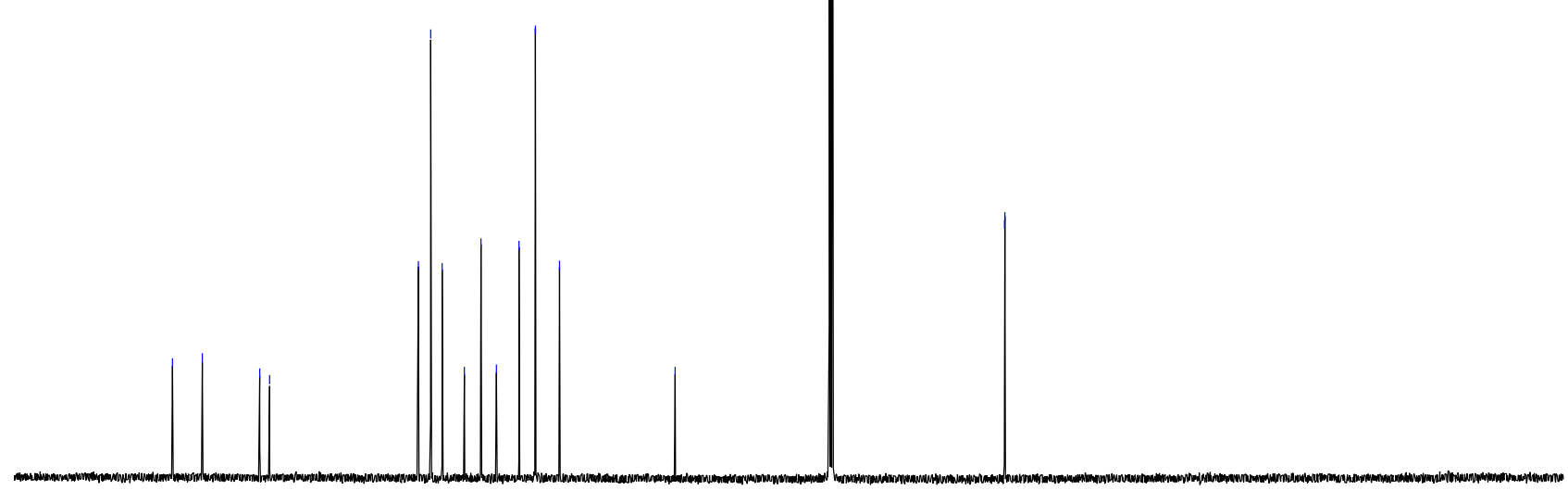

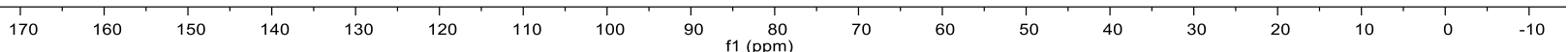




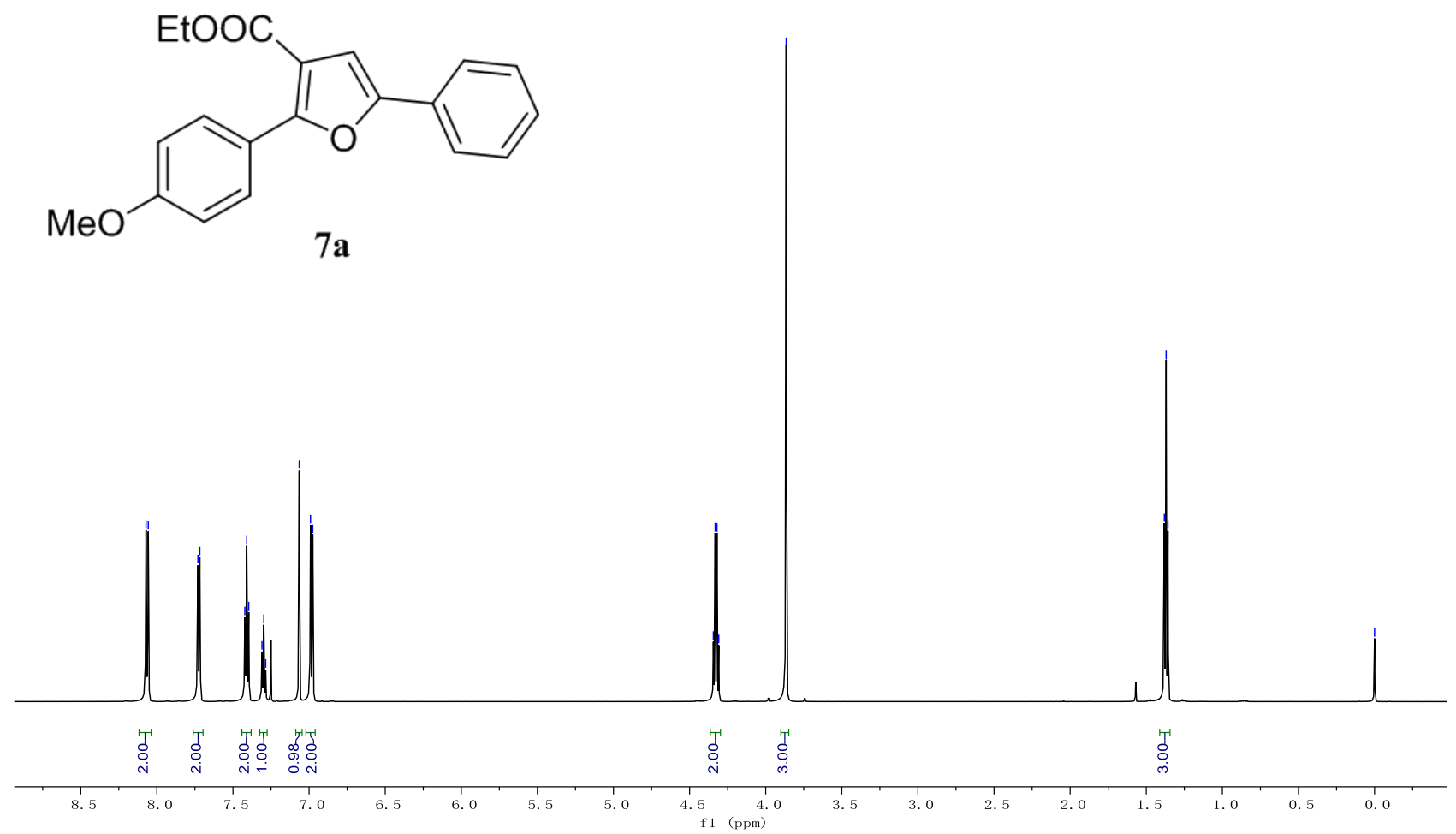

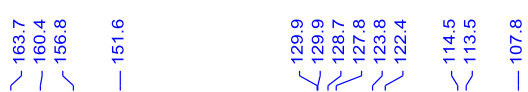

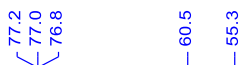

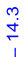
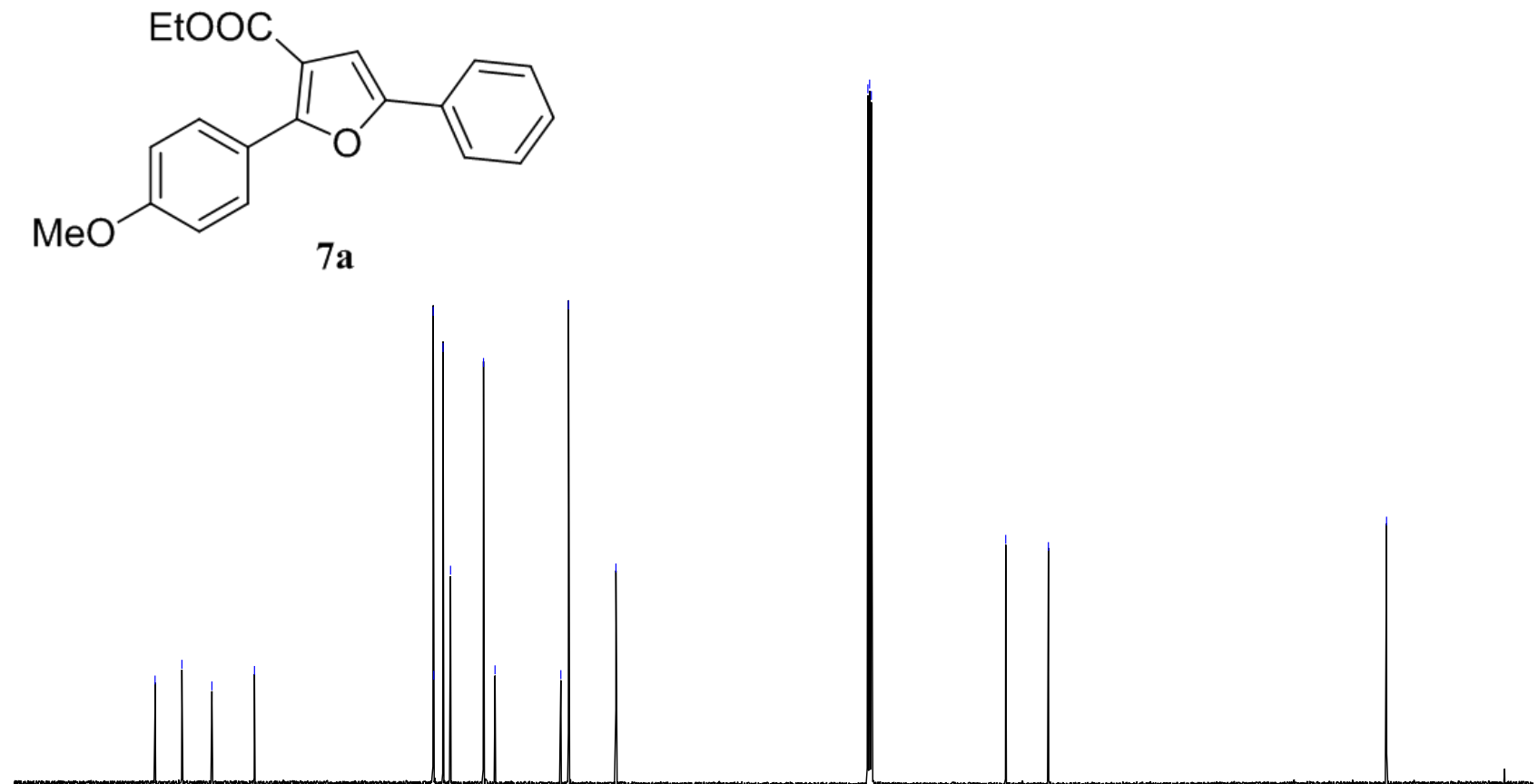

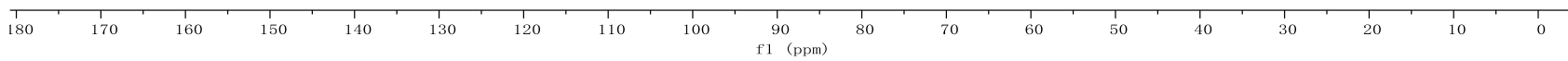



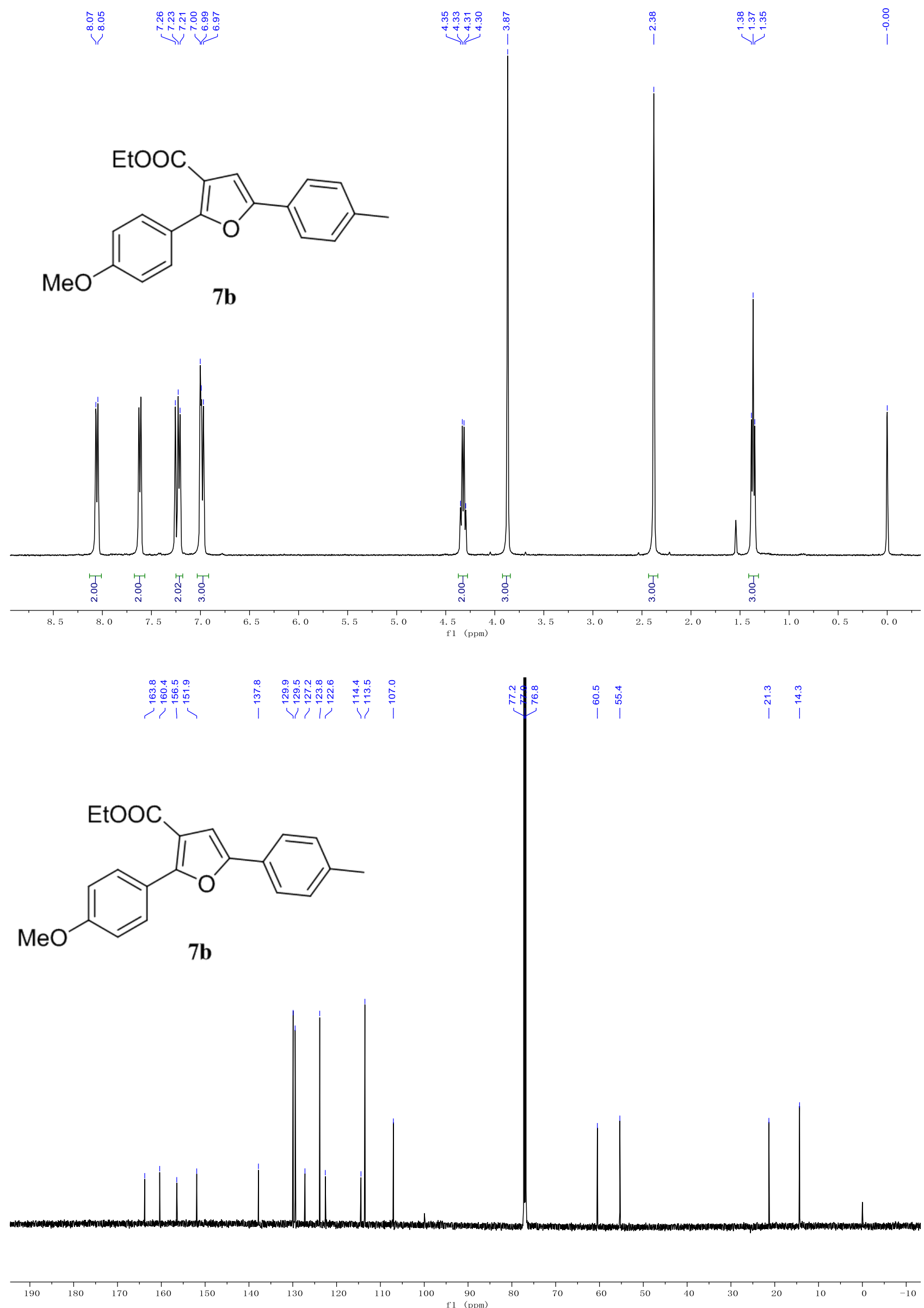

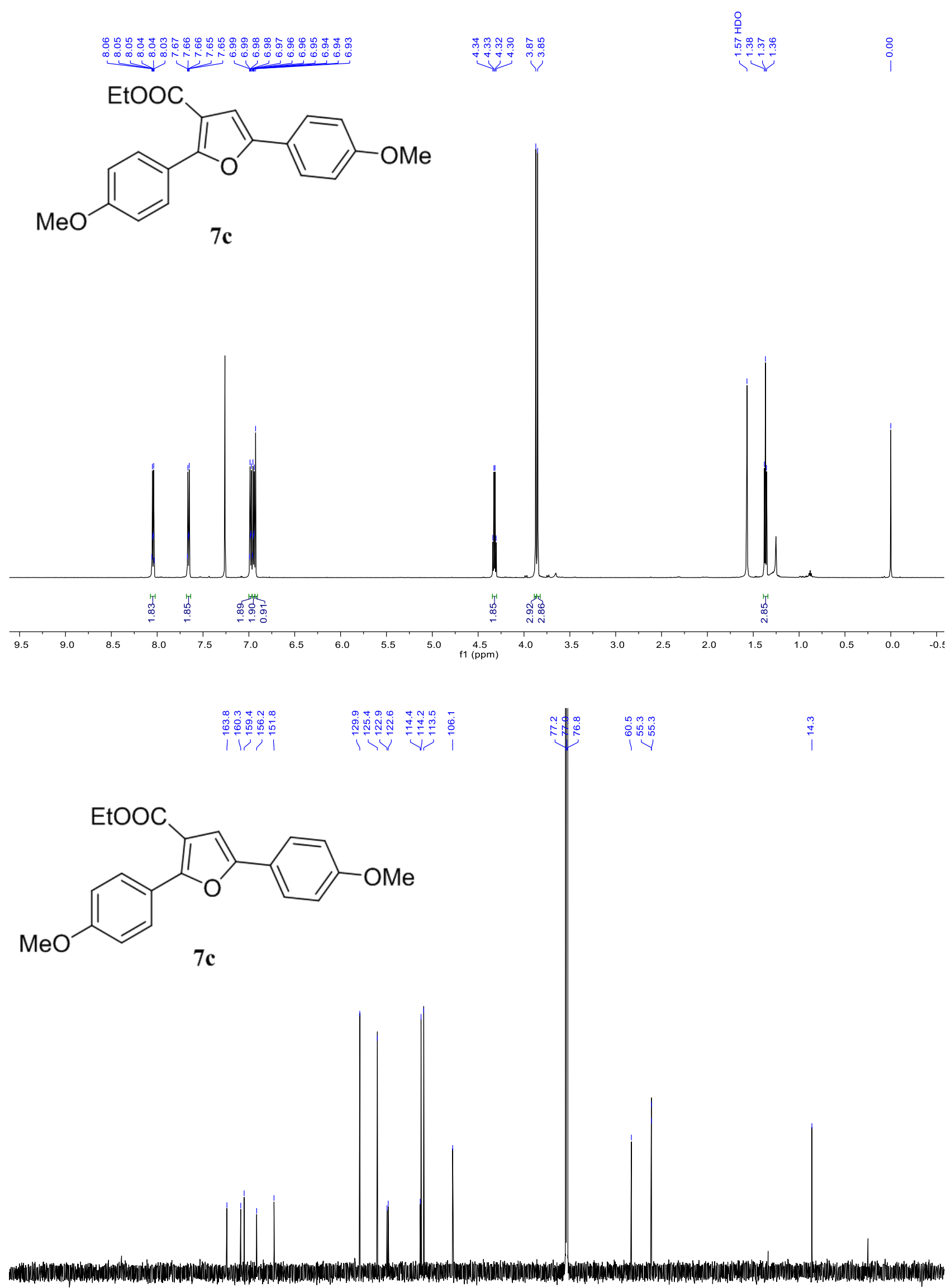

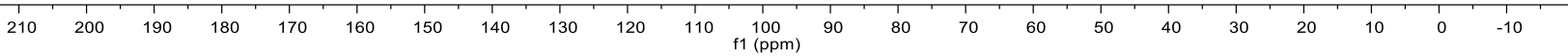


<smiles>CCOC(=O)c1cc(-c2cccc3ccccc23)oc1-c1ccc(OC)cc1</smiles>

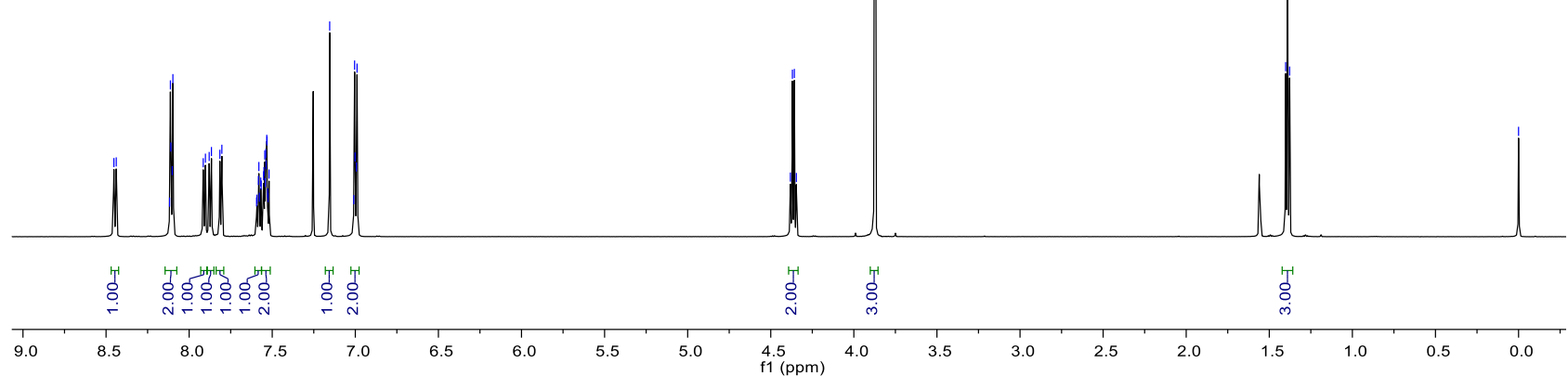

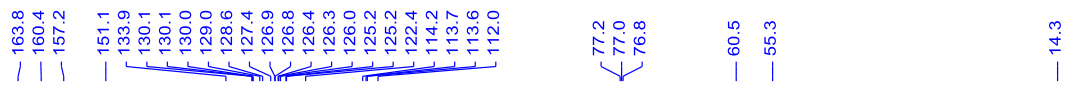<smiles>CCOC(=O)c1cc(-c2cccc3ccccc23)oc1-c1ccc(OC)cc1</smiles>

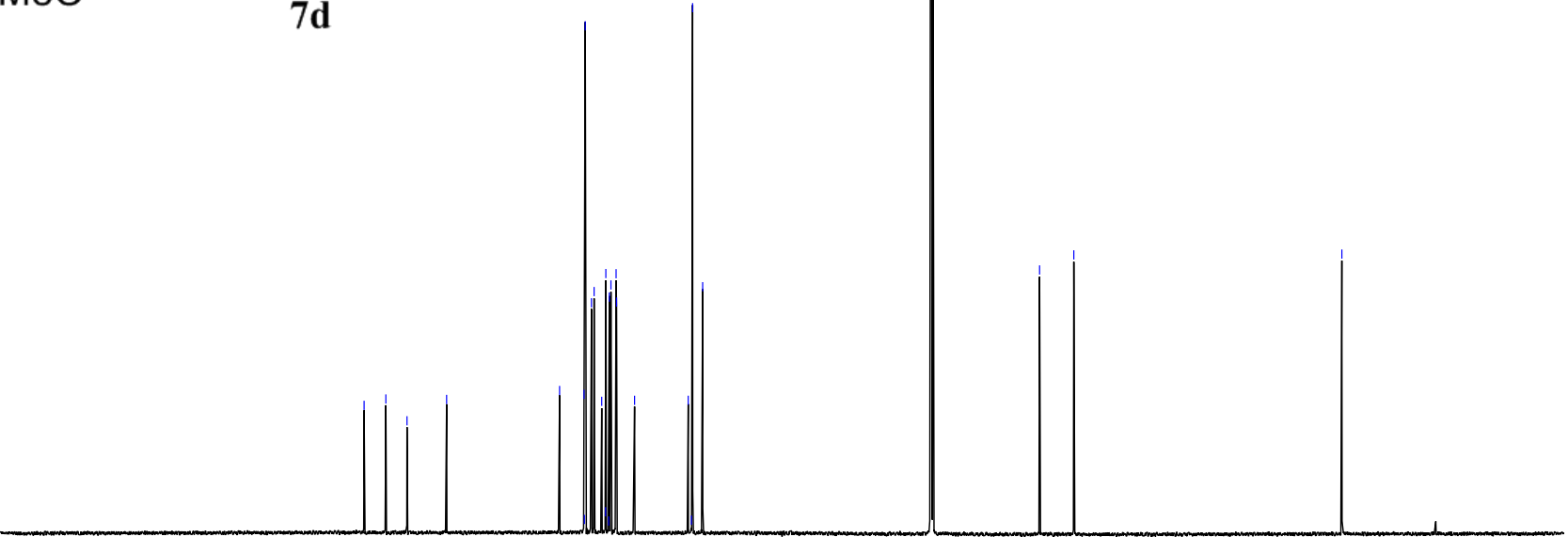



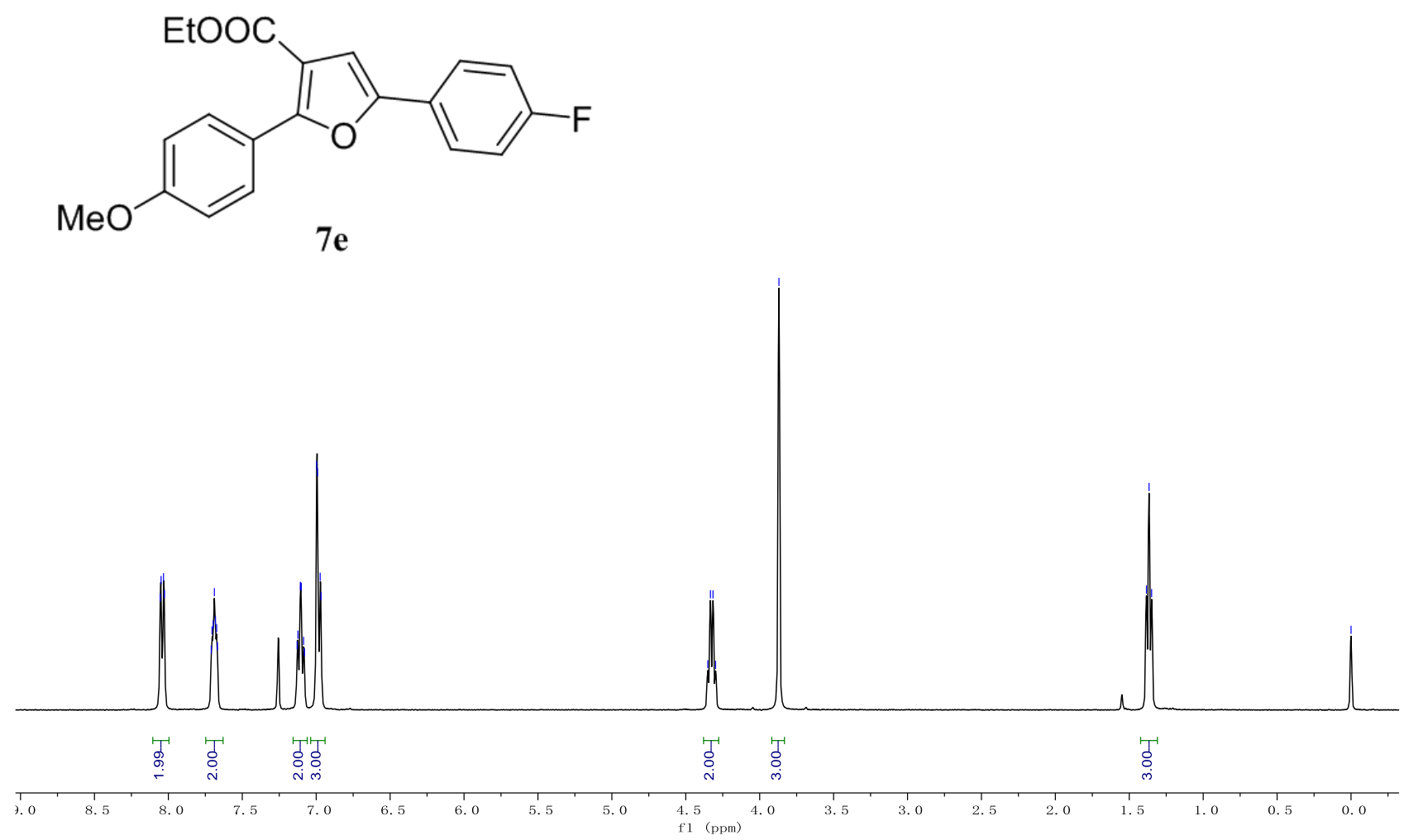

Vil |

$\sqrt{2}$
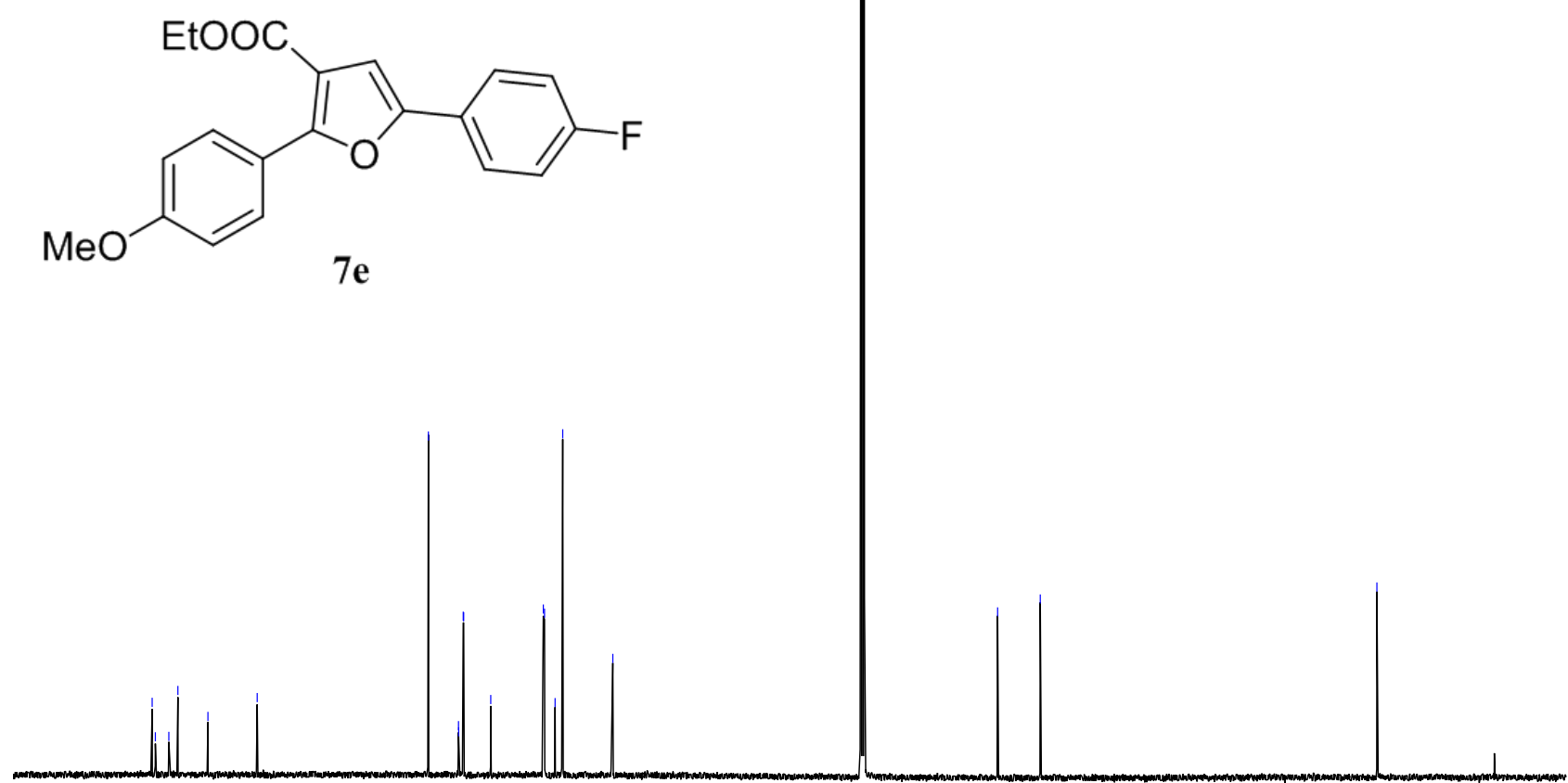

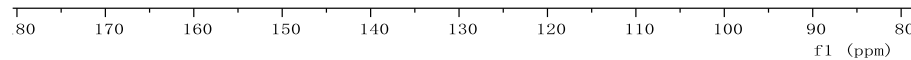



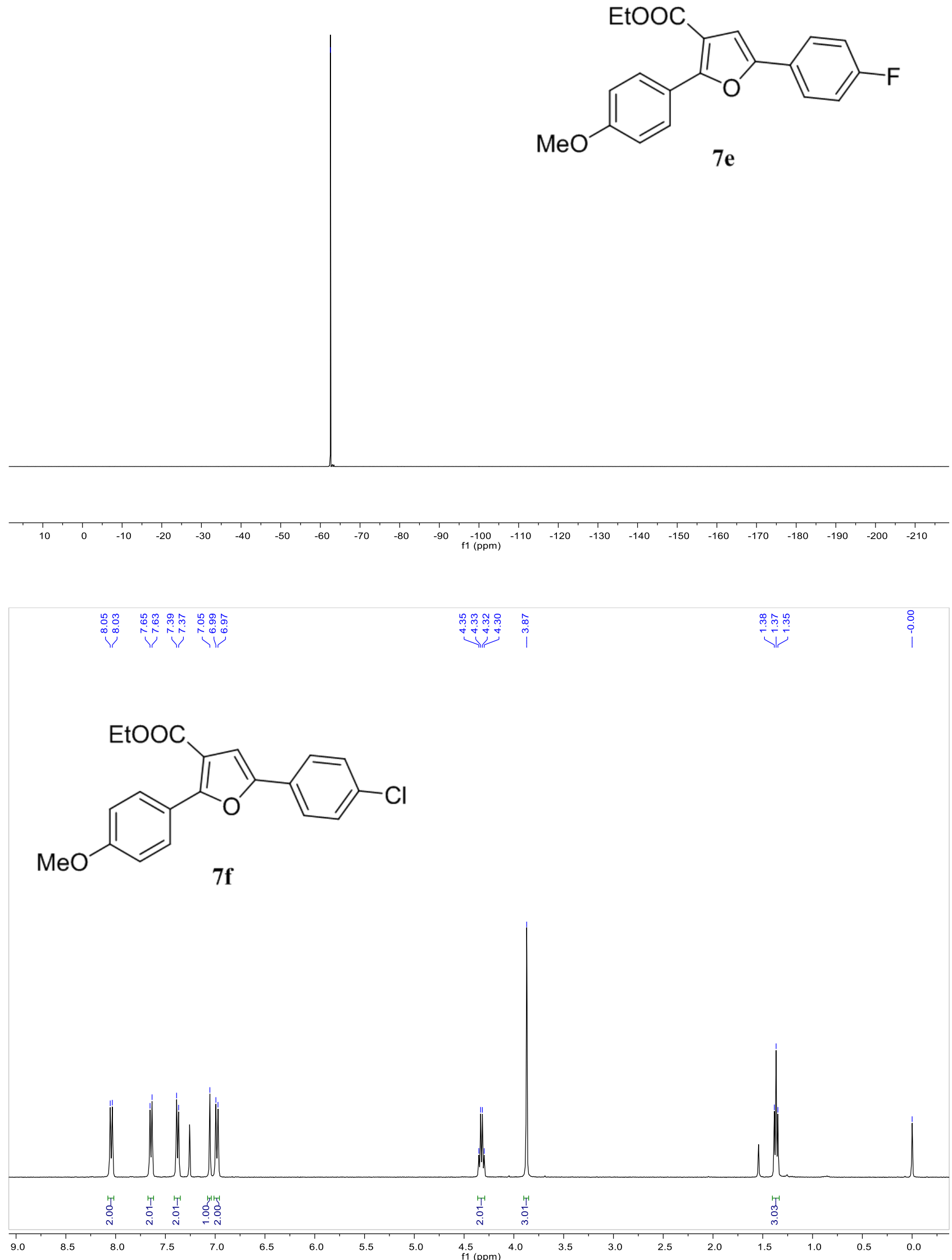

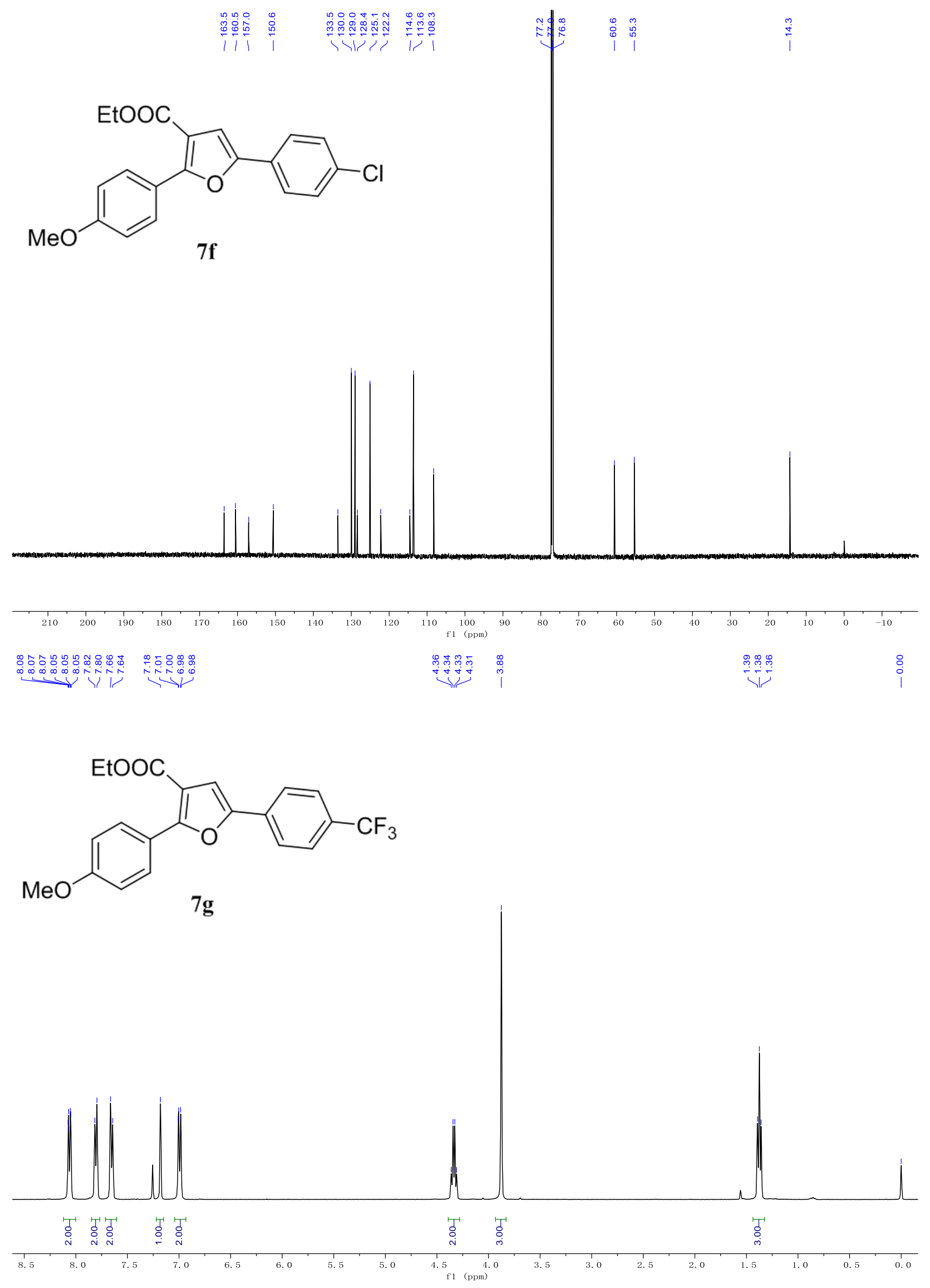


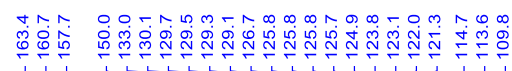

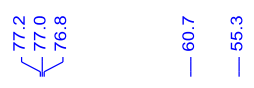
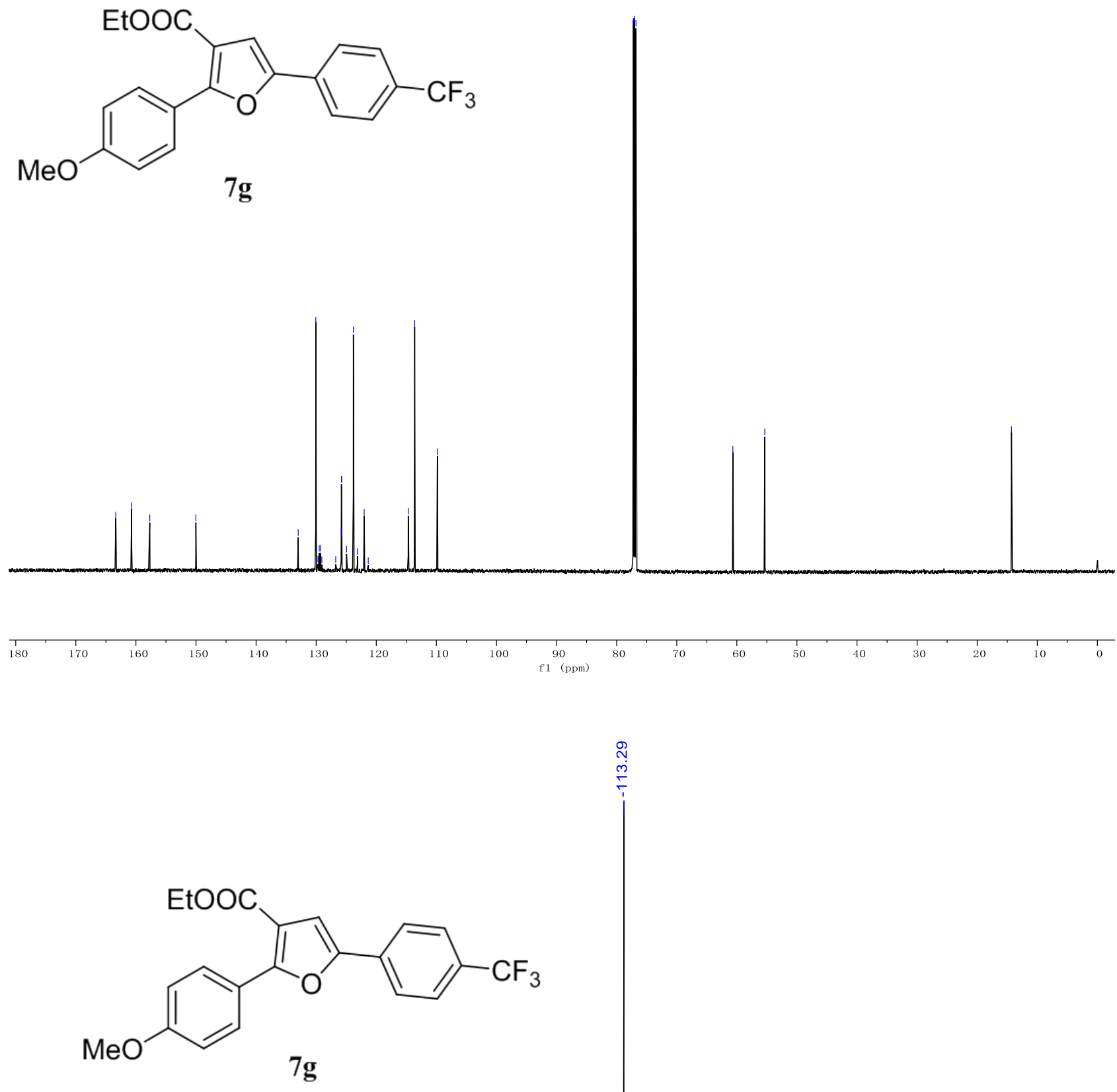

10

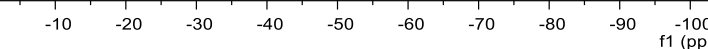


<smiles>CCOC(=O)c1cc(-c2cccc(Cl)c2)oc1-c1ccc(OC)cc1</smiles>

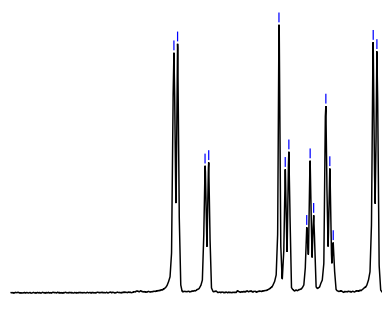

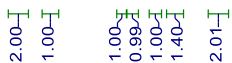

$\Gamma_{1}^{1}$

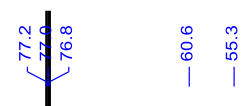<smiles>CCOC(=O)c1cc(-c2cccc(Cl)c2)oc1-c1ccc(OC)cc1</smiles>

$7 h$

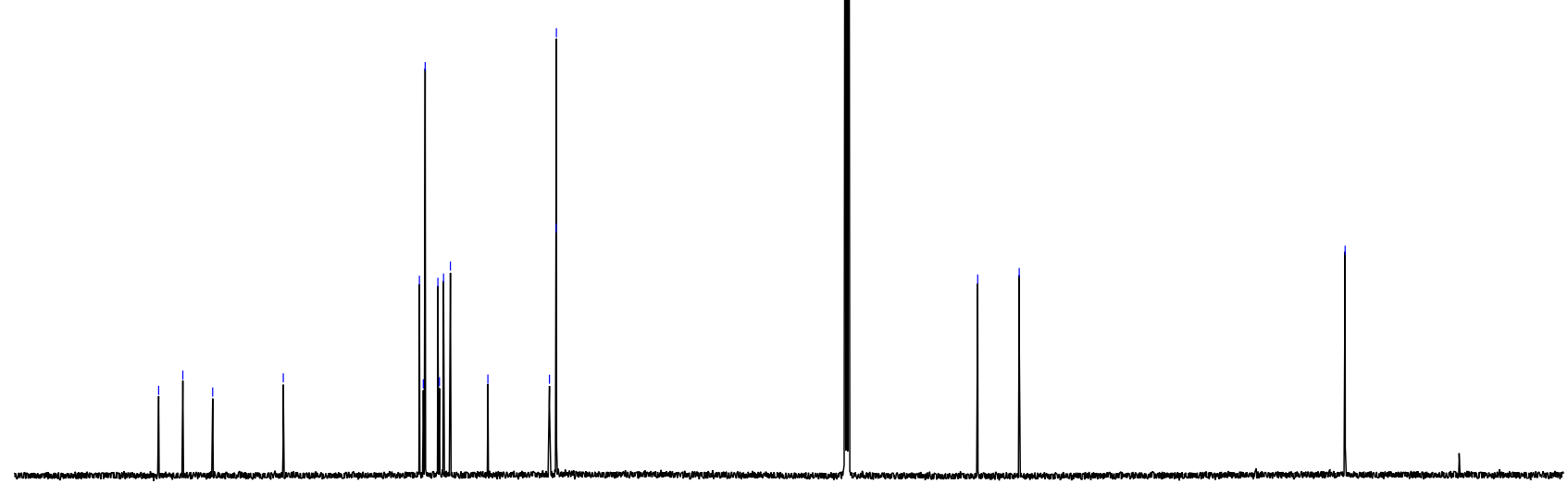

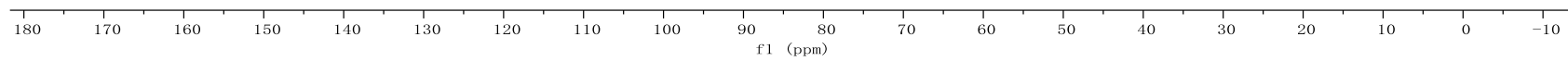



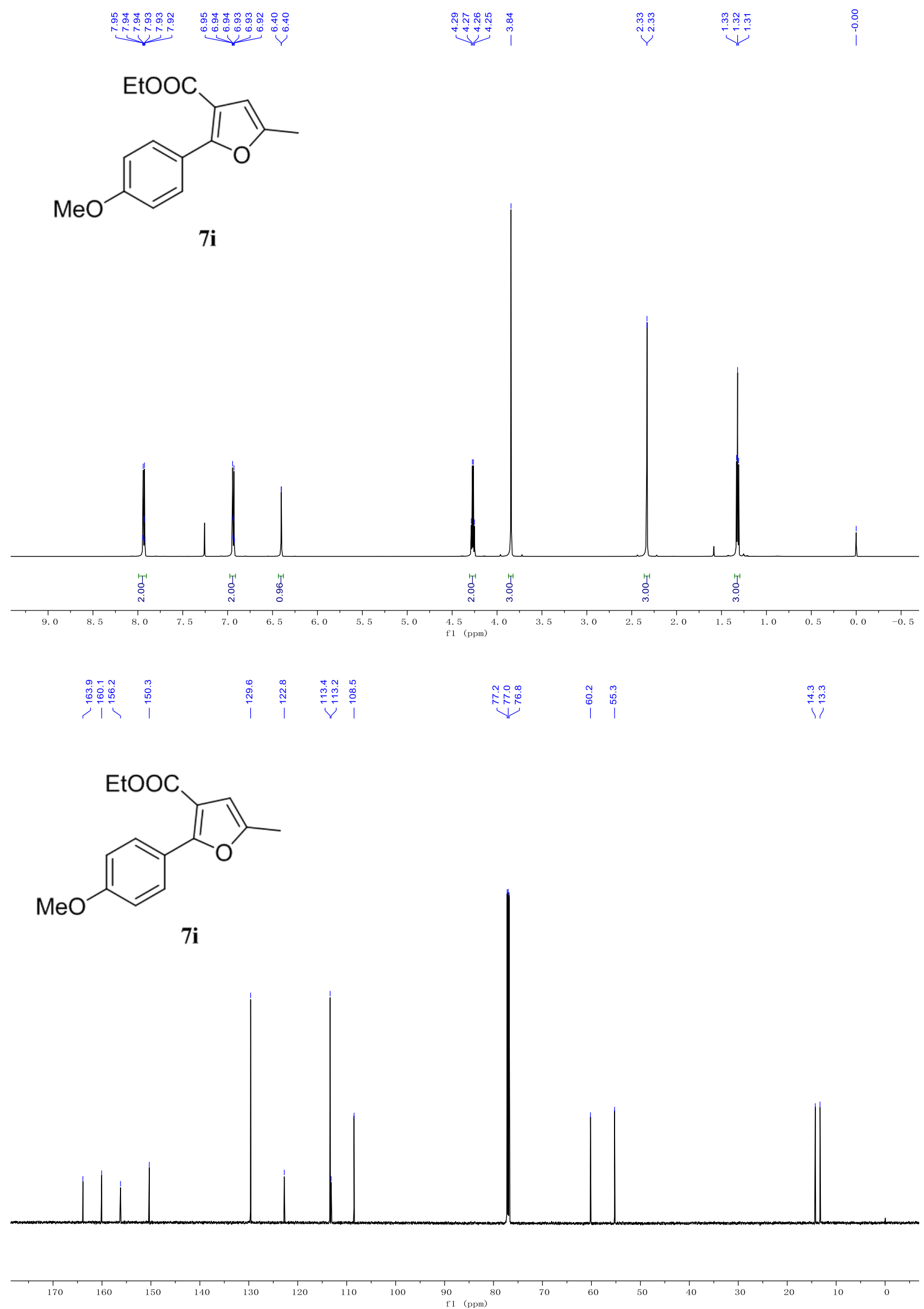


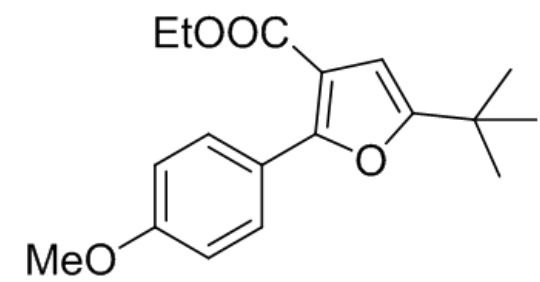

$7 j$

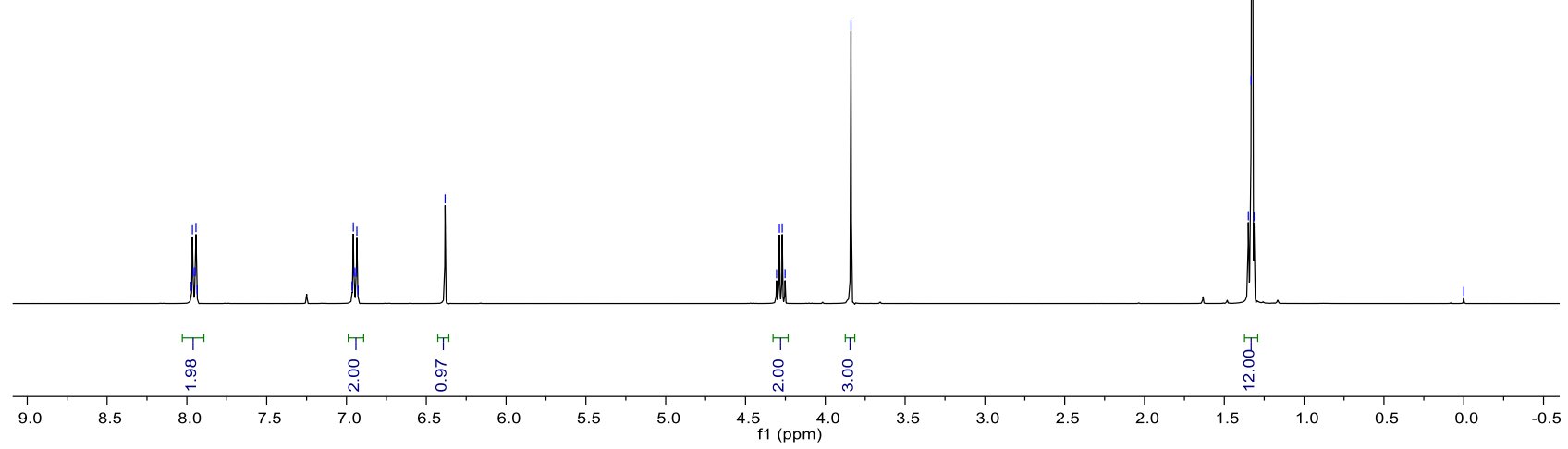

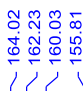

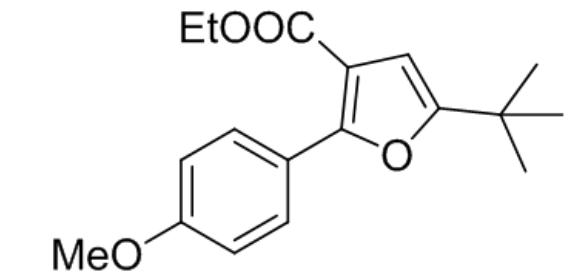

$7 \mathbf{j}$

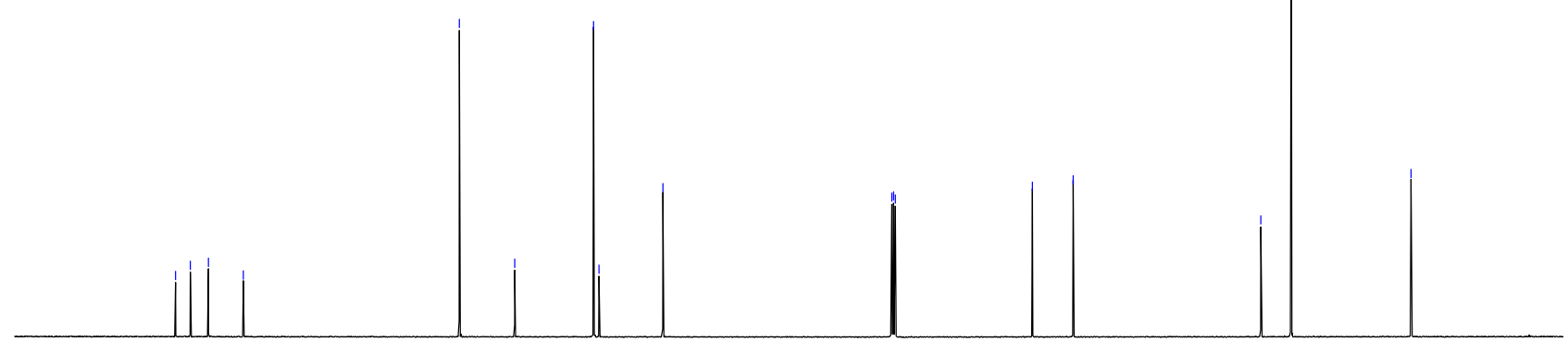

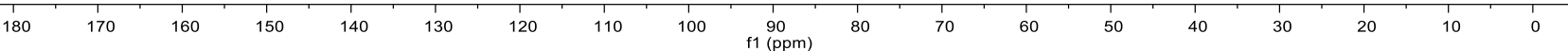




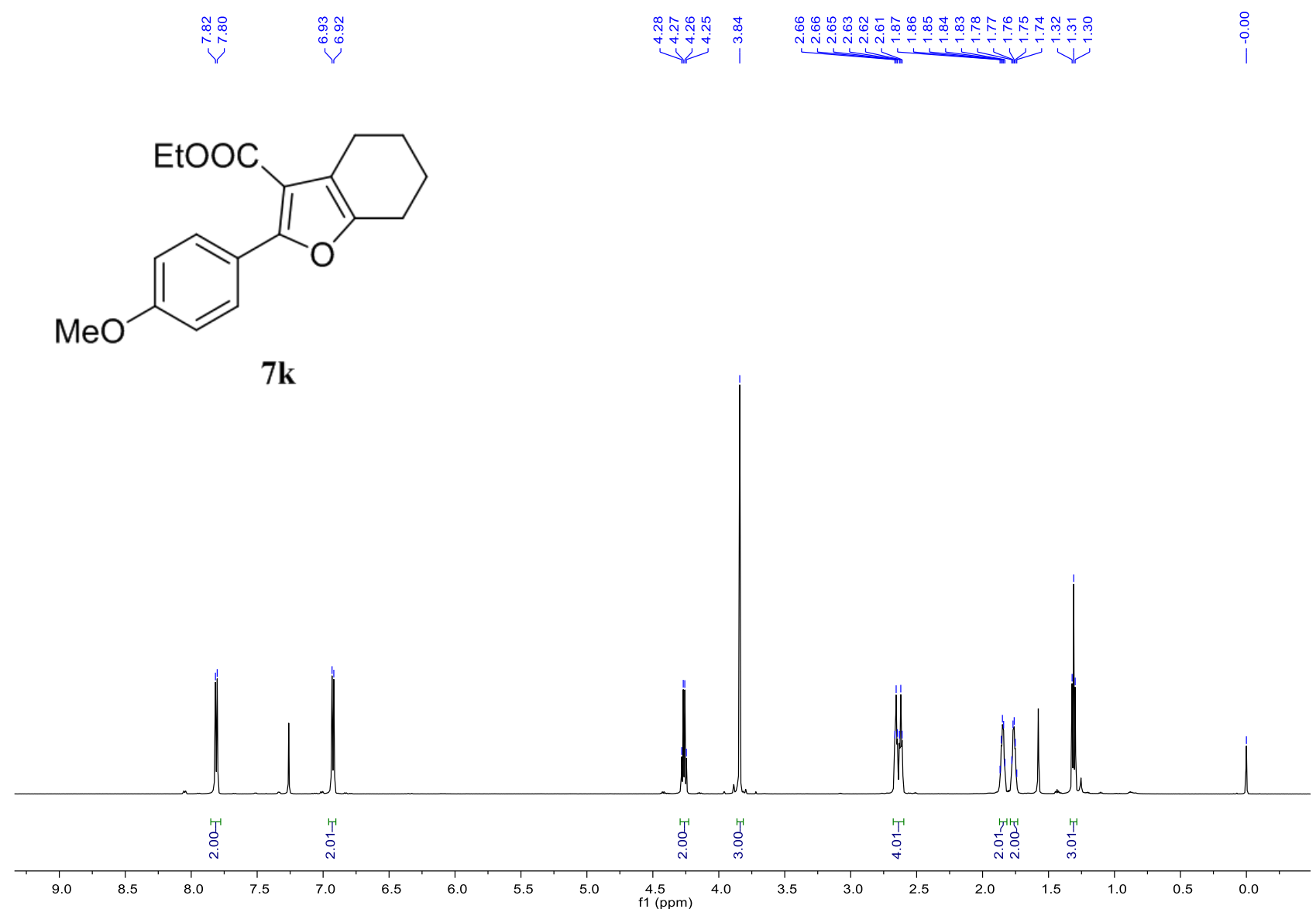

H In

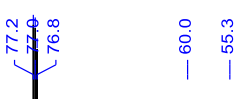

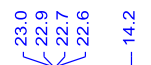<smiles>CCOC(=O)c1c(-c2ccc(OC)cc2)oc2c1CCCC2</smiles>

$7 k$

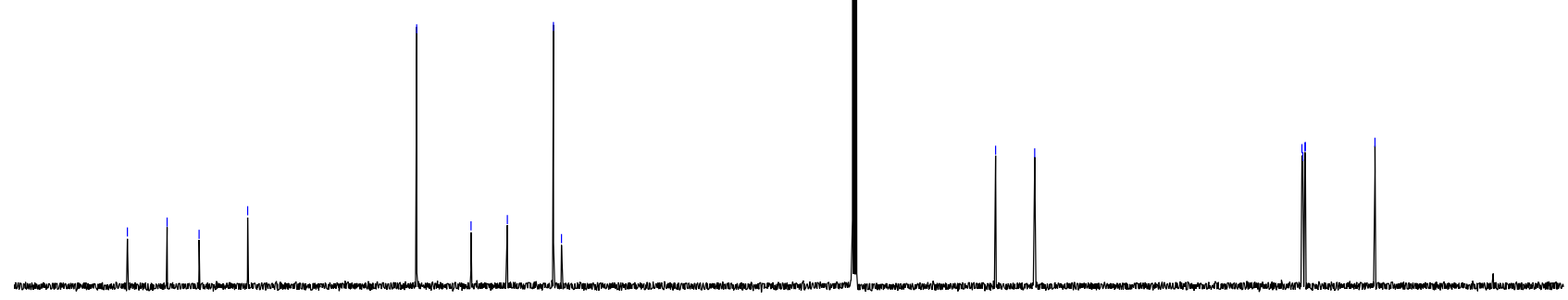

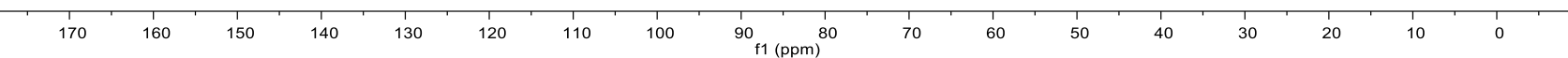


<smiles>[2H]C1([2H])[C@H](C(=O)c2ccccc2)[C@H]1c1ccc(OC)cc1</smiles>
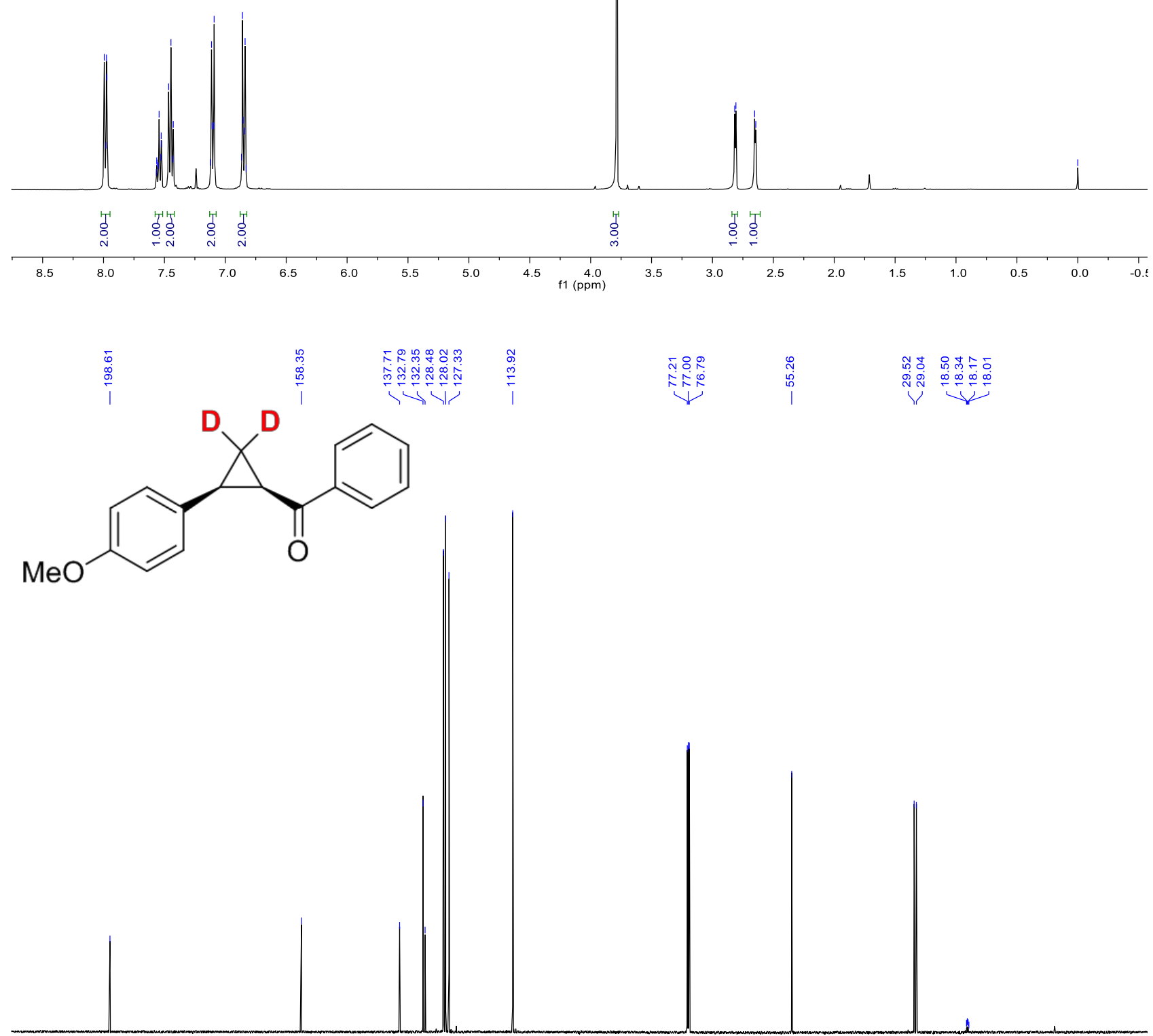

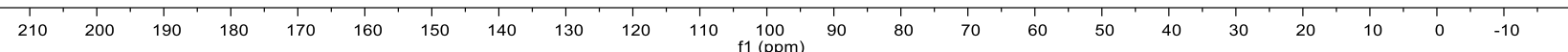



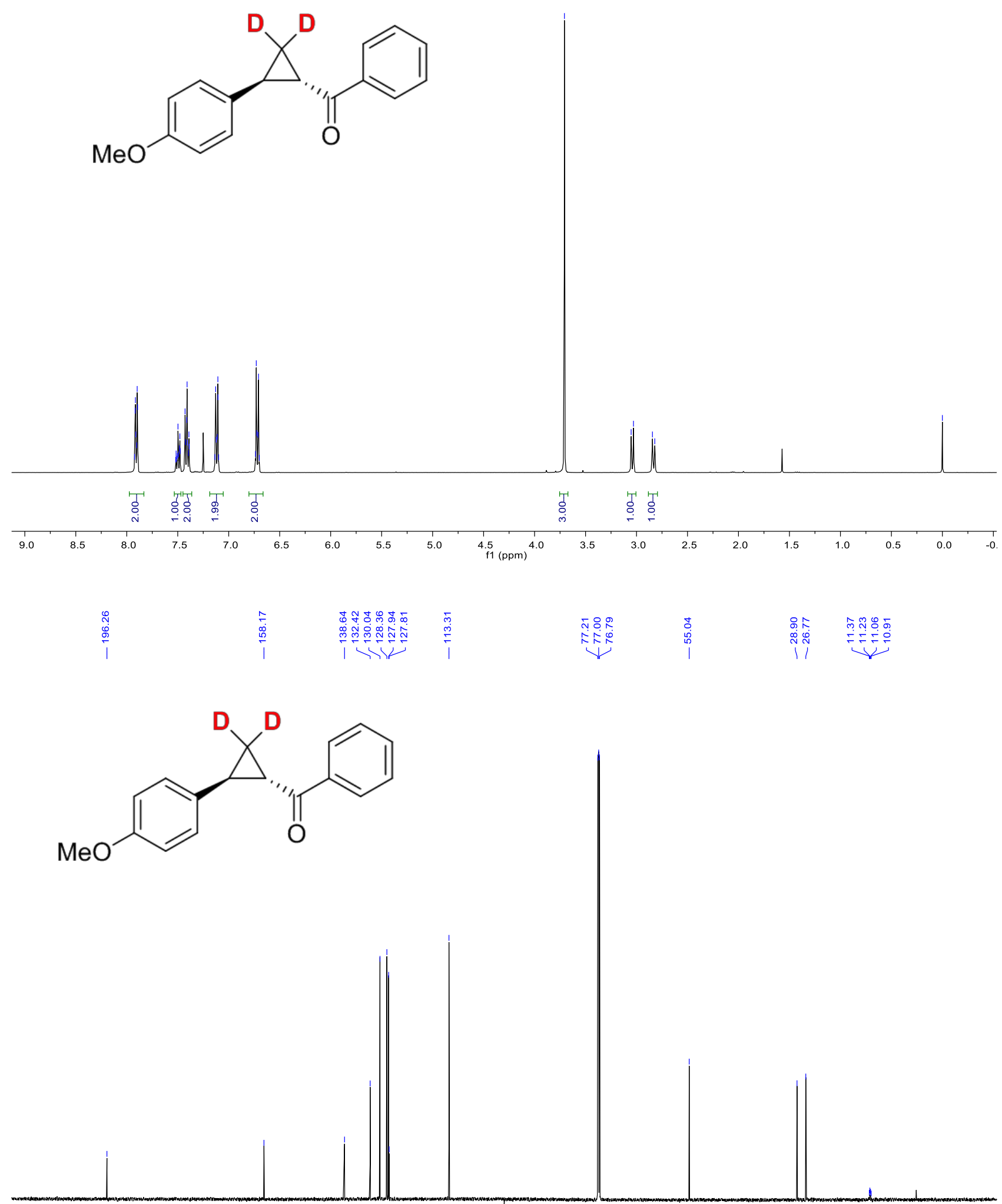

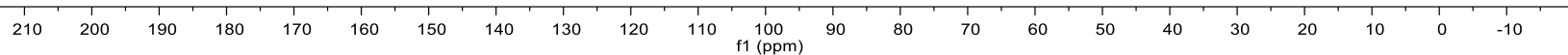



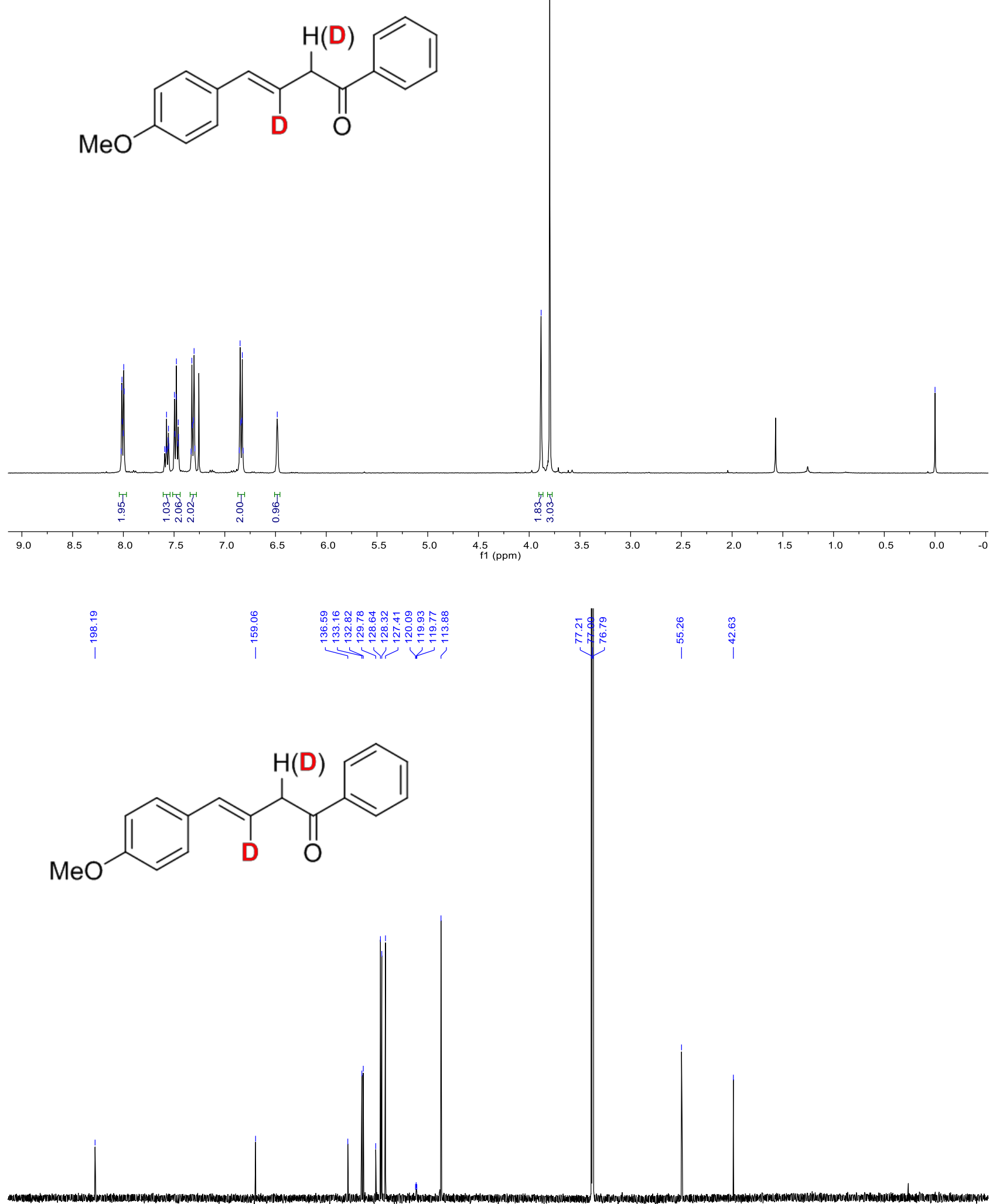

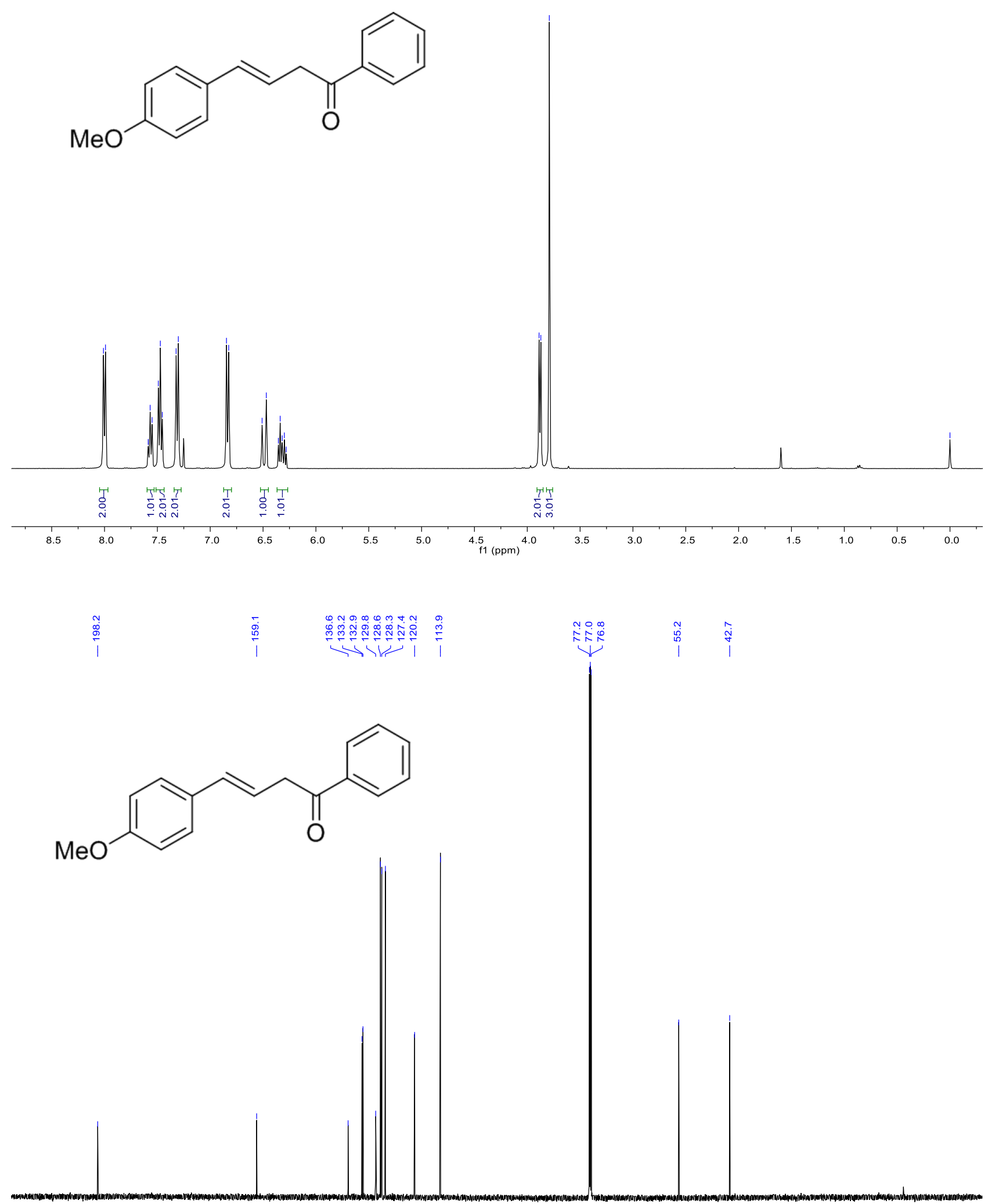

$\begin{array}{lllllllllllllllllllllllllllllll}1 & 210 & 200 & 190 & 180 & 170 & 160 & 150 & 140 & 130 & 120 & 110 & 100 & 90 & 80 & 70 & 60 & 50 & 40 & 30 & 20 & 10 & 0 & -10 & 1\end{array}$ 